\title{
AGED 539 \\ Teacher Internship Report
}

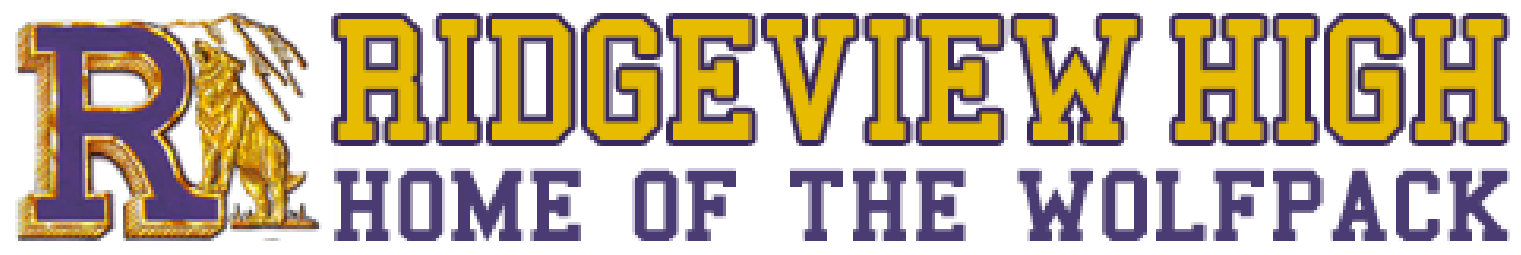

Ridgeview High School Agriculture Department Jenny Potterton 
AGED 539

Teacher Internship Report Contents

I Project - Program of Work

II Quality Criteria Narratives

III Supporting Completion Materials

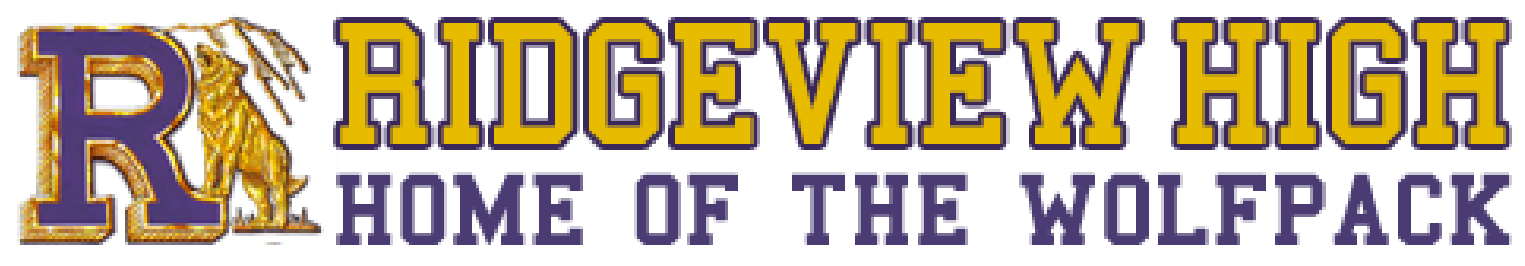



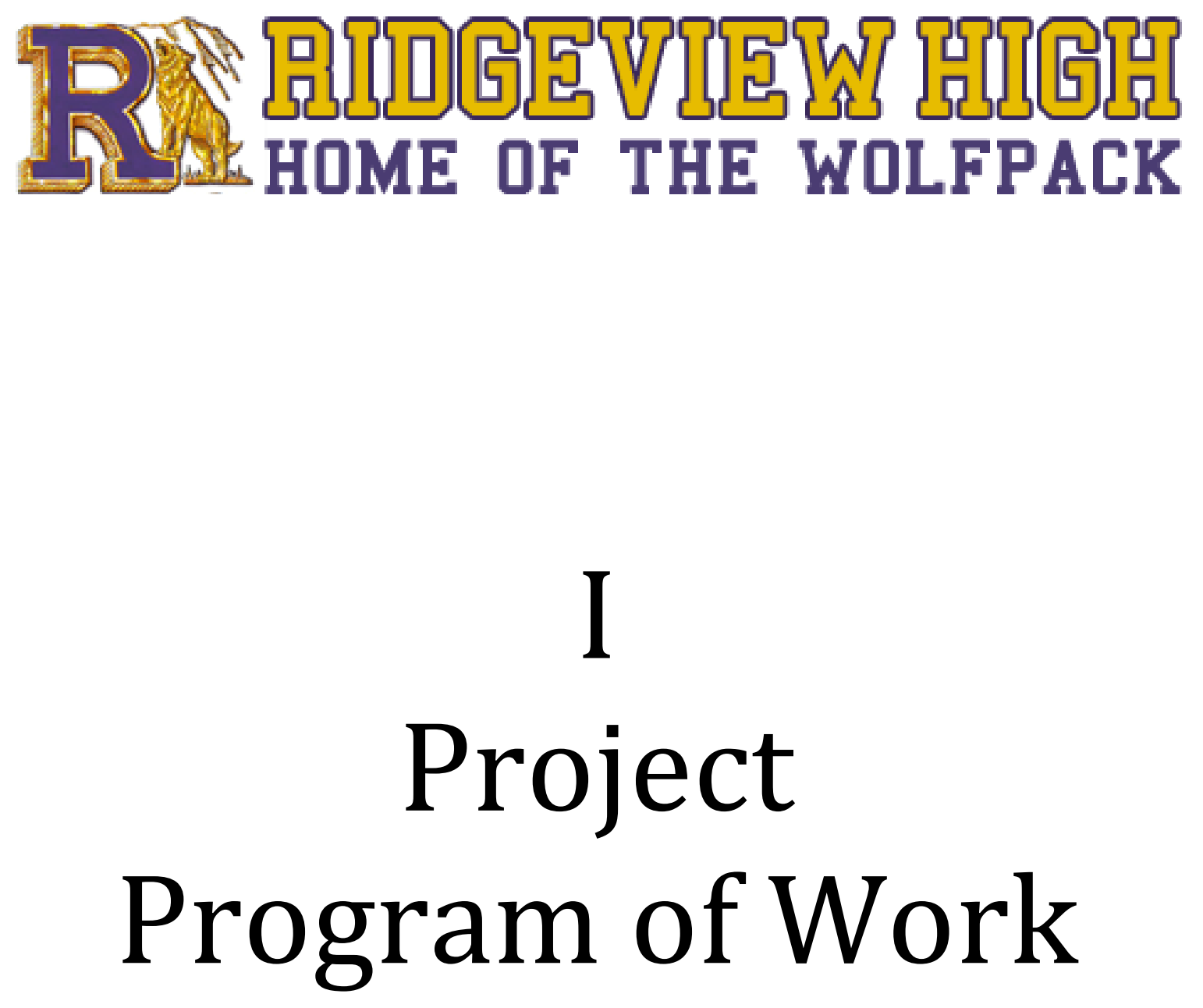


\section{Project Proposal}

(to be completed in conjunction with AGED 539)

Quality Criteria Number Addressed: 9 .

Goal or Purpose of the Project:

Create a Program of Activities for Ridgeview FFA.

Specific Objectives to Accomplish (Be as detailed as possible):

To update very old POAs s that the chapter has an updated version.

Estimated number of hours on this project: 15

Estimated expenditures (\$) on this project (your costs): 0

Proposed timeline for completion of the project:

Spring 2015

Progress Report: How will you inform the Cal Poly faculty of your progress on a regular basis? Communicate updates through email 


\section{Program of Activities}

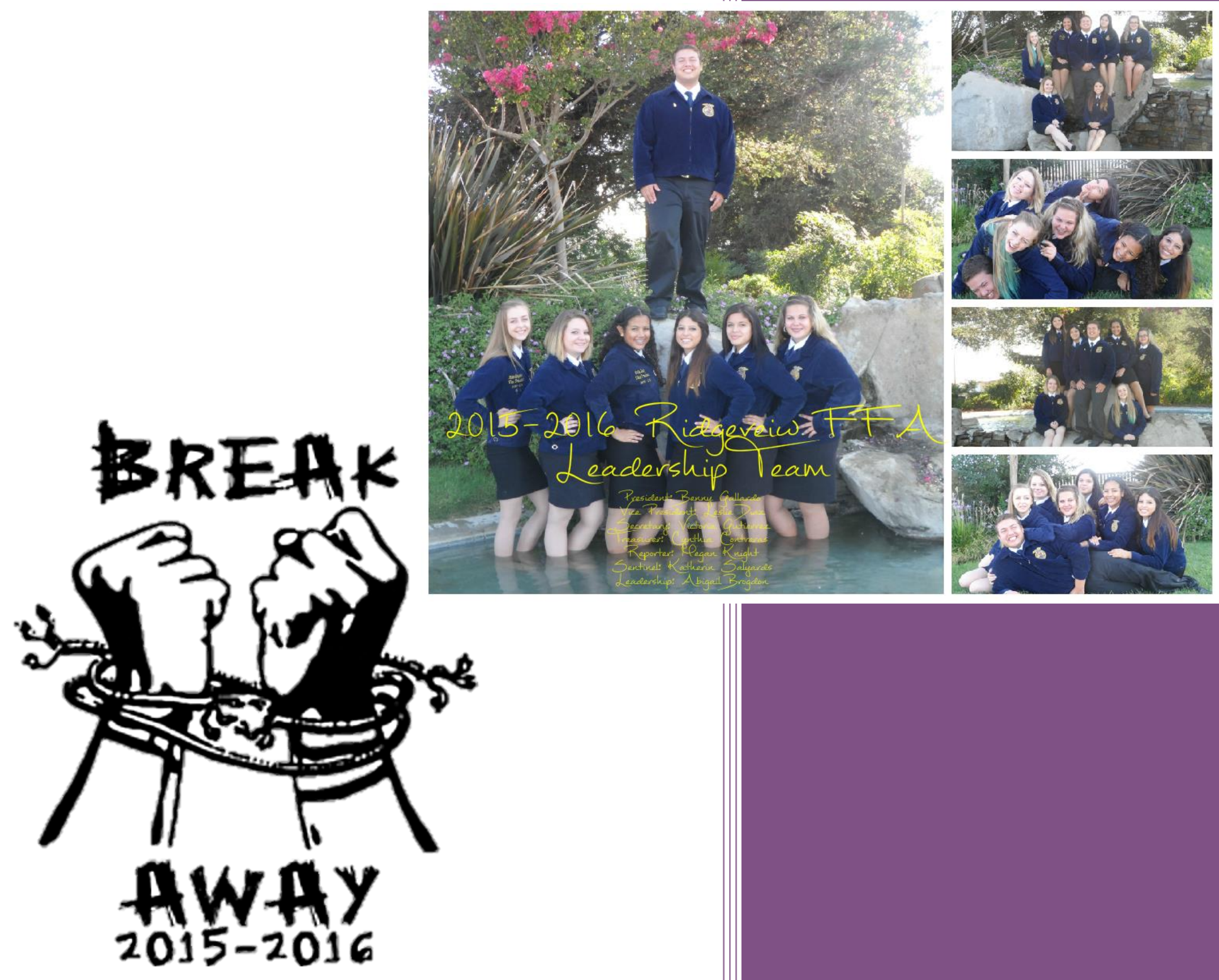




\section{Contents:}

Introduction

How to Be an Active FFA Member

Chapter Leadership

Calendar of Activities

The Aims \& Purposes of the FFA Organization

FFA Colors

FFA Motto

The FFA Emblem

The FFA Creed

Official FFA Uniform

Proper Use of the FFA Jacket

FFA Code of Ethics

Career Development Events

More Opportunities to Stay Involved

SAE

Proficiency Awards

Leadership

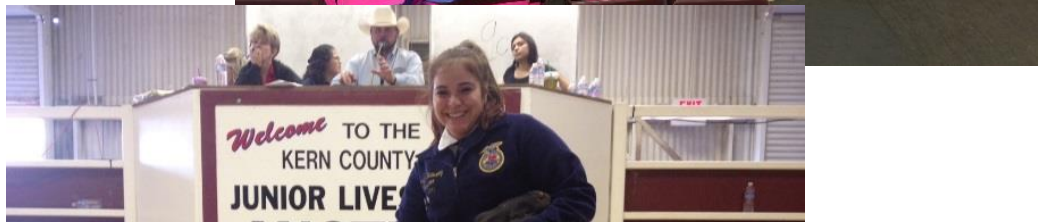

Conduct of Meetings

Scholarships

Rules and Conditions Governing FFA Trips

Fairs

Point Awards System

Historical Highlights

Constitution of the Bakersfield-Ridgeview FFA Chapter

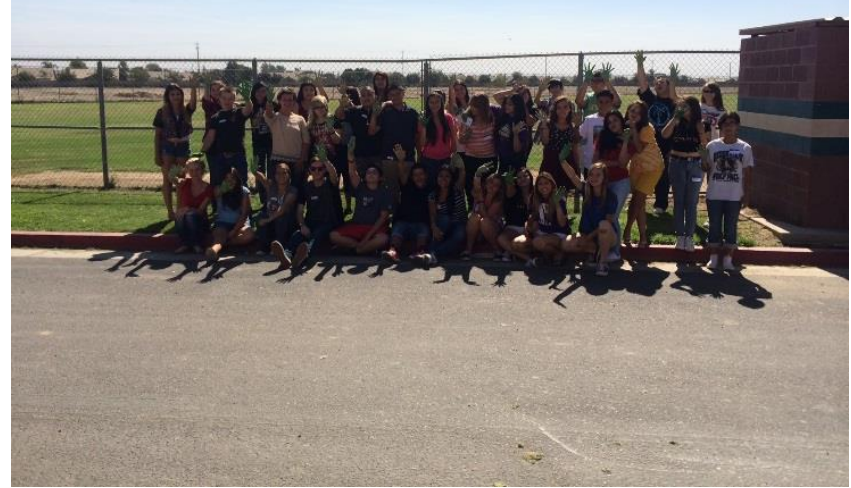




\section{Introduction}

The FFA Organization is an organization of, by, and for students studying agriculture in public secondary schools under the provision of the National Vocational Education Acts.

As an integral part of the program of agriculture education in the public school system of America, the FFA has become well known in recent years. No national student organization enjoys greater freedom of self-government under adult council and guidance than the FFA. Organized in November 1928, it has served to motivate and vitalize the effective instruction offered to students of vocation agriculture and to provide further training in farmer citizenship and agricultural business.

The FFA is a nonprofit, nonpolitical agriculture youth organization, designed to take its place among other agents striving for the development of leadership, the advancement of agricultural technology and improvement of agricultural life. The foundation upon which the FFA organization is molded includes leadership, service, thrift, scholarship, improved agriculture, organized recreation, citizenship, and patriotism.

National headquarters for the FFA is located in the Agricultural Education Branch Office of Health, Education, and welfare in Washington D.C. The National Convention is held annually in Louisville, Kentucky and the California Association holds its annual Convention at the Fresno Convention Center, Fresno, California.

This 2014-2015 Program of Work was developed to explain the purpose of the FFA Organization and give insight into the opportunities that are available to all students of agriculture a Ridgeview High School.
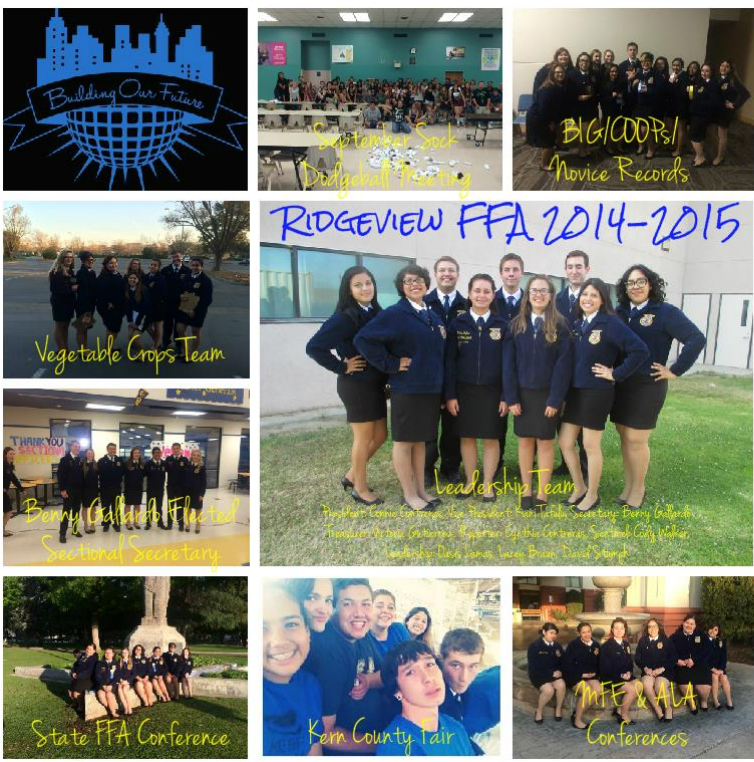
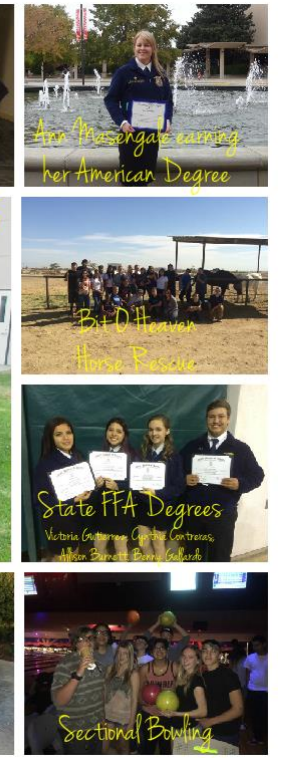


\section{How to Be an Active FFA Member}

An active FFA member of the Ridgeview FFA chapter is one who:

1. Is enrolled in an Agriculture Education class

2. Has paid their dues at the local, state, and national levels

3. Maintains a 2.0 or higher grade point average at all times

4. Earns a " $\mathrm{C}$ " or better in an Agriculture course

5. Has earned necessary points when applicable

6. Participate in fundraisers

7. Has become involved in a Supervised Agriculture Experience project

Every student enrolled in an Agriculture Education course at Ridgeview High School is eligible to join FFA. The first step is to sign up which starts the process of active membership.

The next step is to maintain a 2.0 or higher G.P.A. If a student falls below a 2.0 for two consecutive grading periods, the student will not be eligible to attend meetings or participate in any FFA activities until eligibility is met.

The final step is to be involved as much as possible. The FFA has a Point Award System and every activity has a point value. At the end of the year, chapter officers grade these points. This information determines the top 10 participating freshmen, sophomores, juniors, and seniors who will be awarded at the Spring chapter banquet.

We encourage everyone to involve him/herself in the FFA program. There are opportunities, which develop leadership, confidence, and friendships. There is a large area for growth within the FFA program.

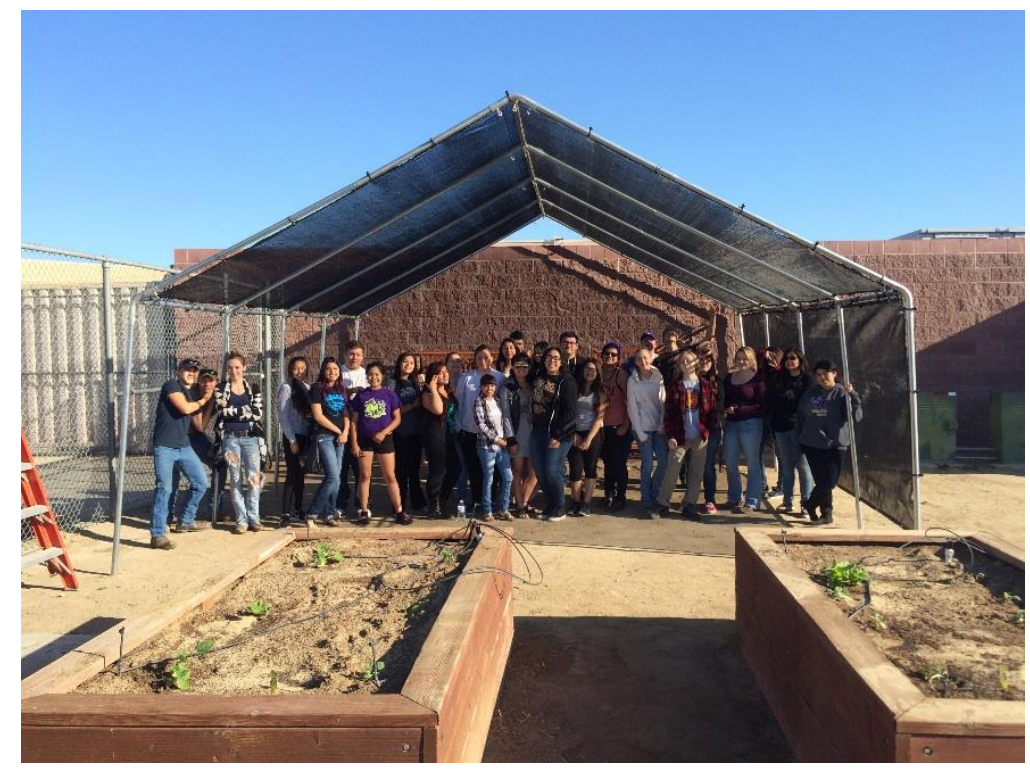




\section{Chapter Leadership}
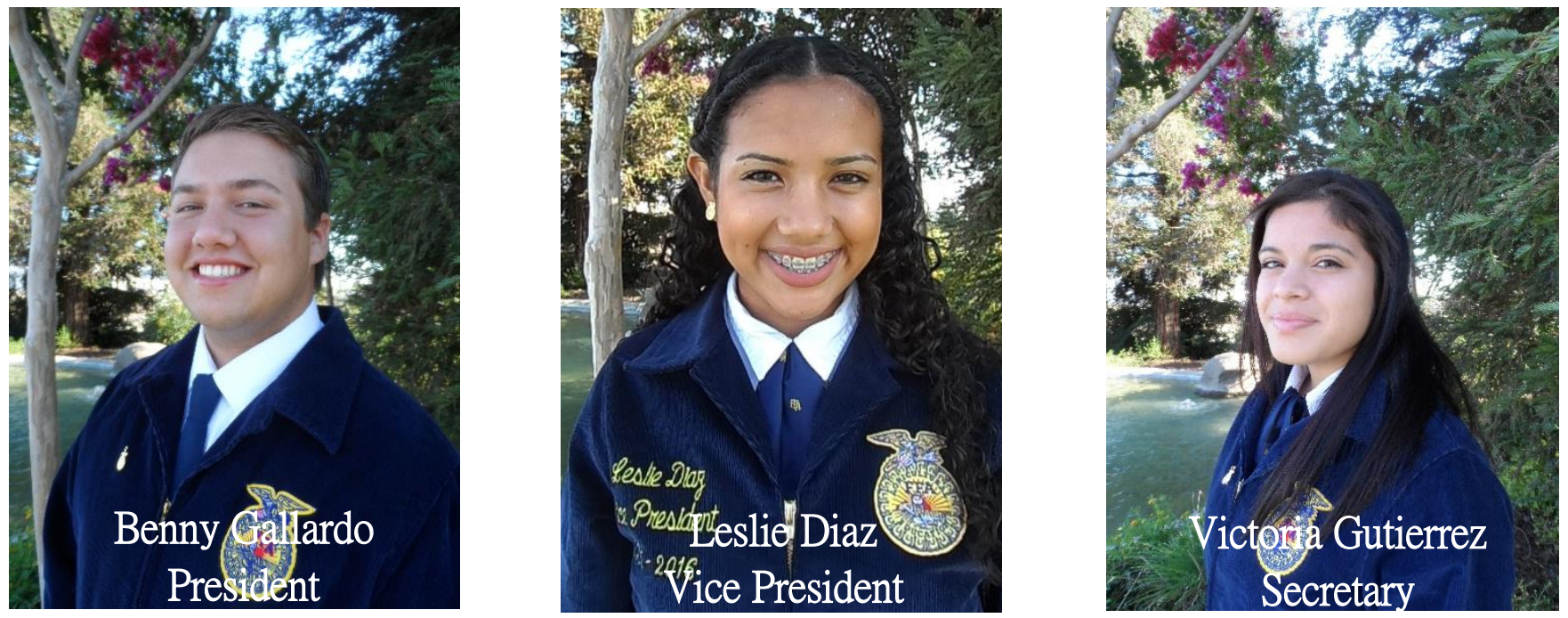
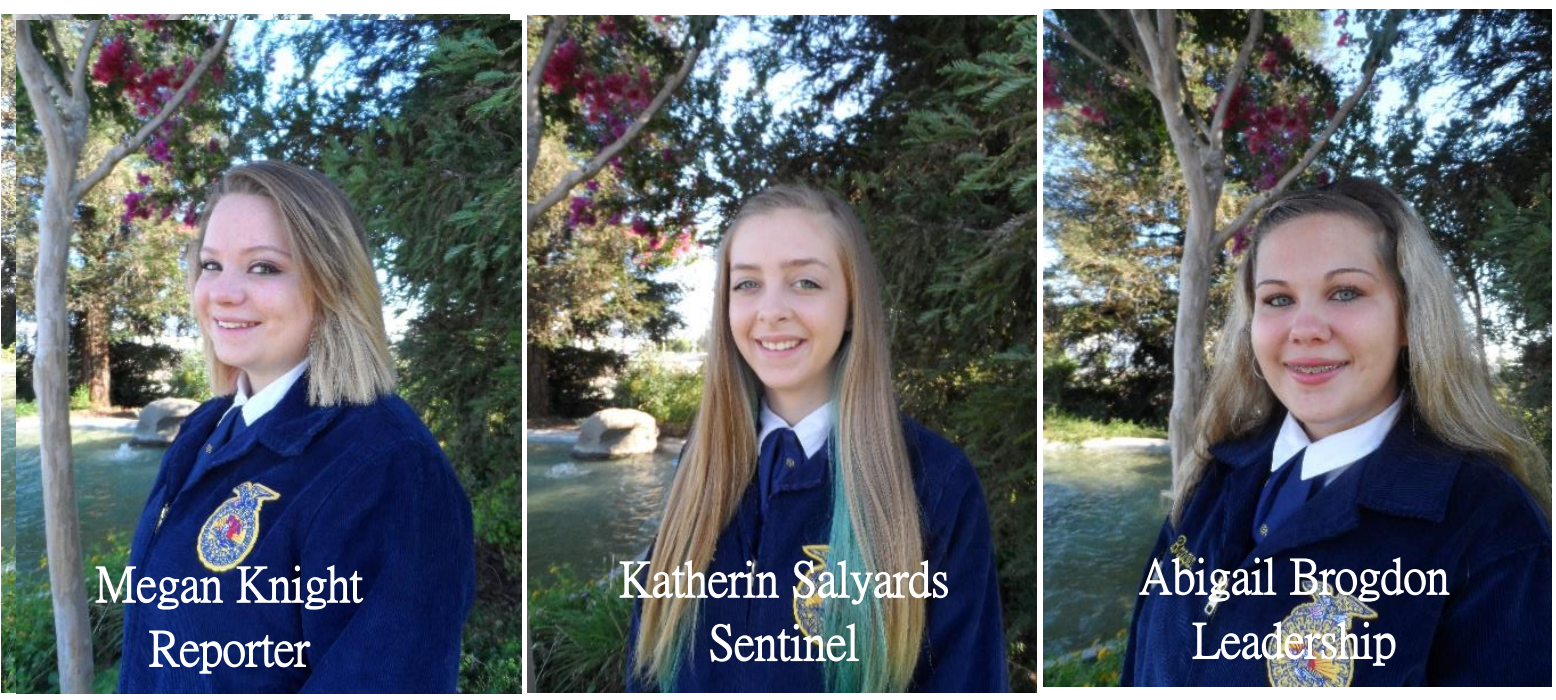

4| P a g e
Ridgeview FFA Program of Activities 


\section{Calendar of Activities}

For the most up to date calendar, visit Potterton.weebly.com
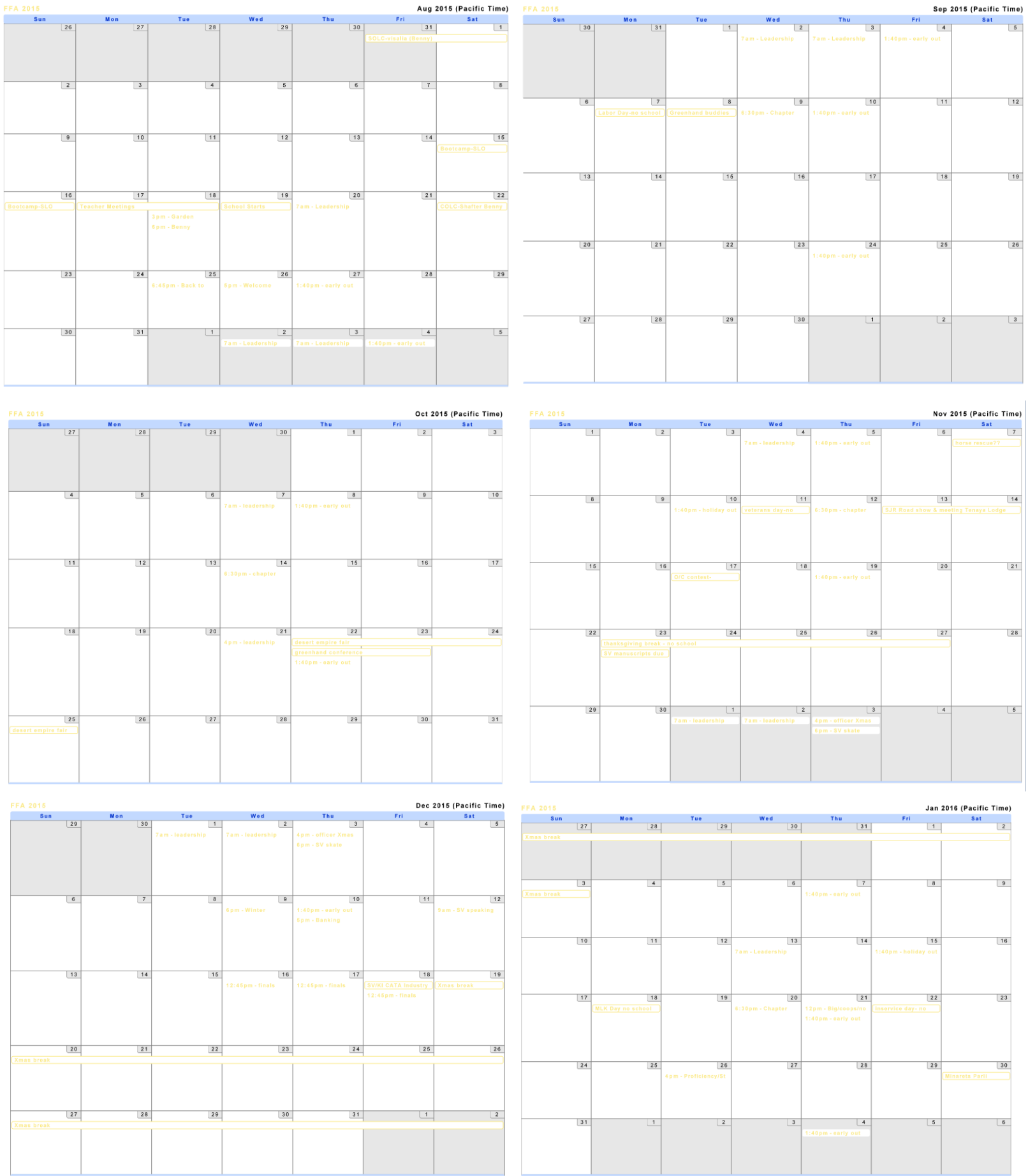

5 | P a g e

Ridgeview FFA Program of Activities 

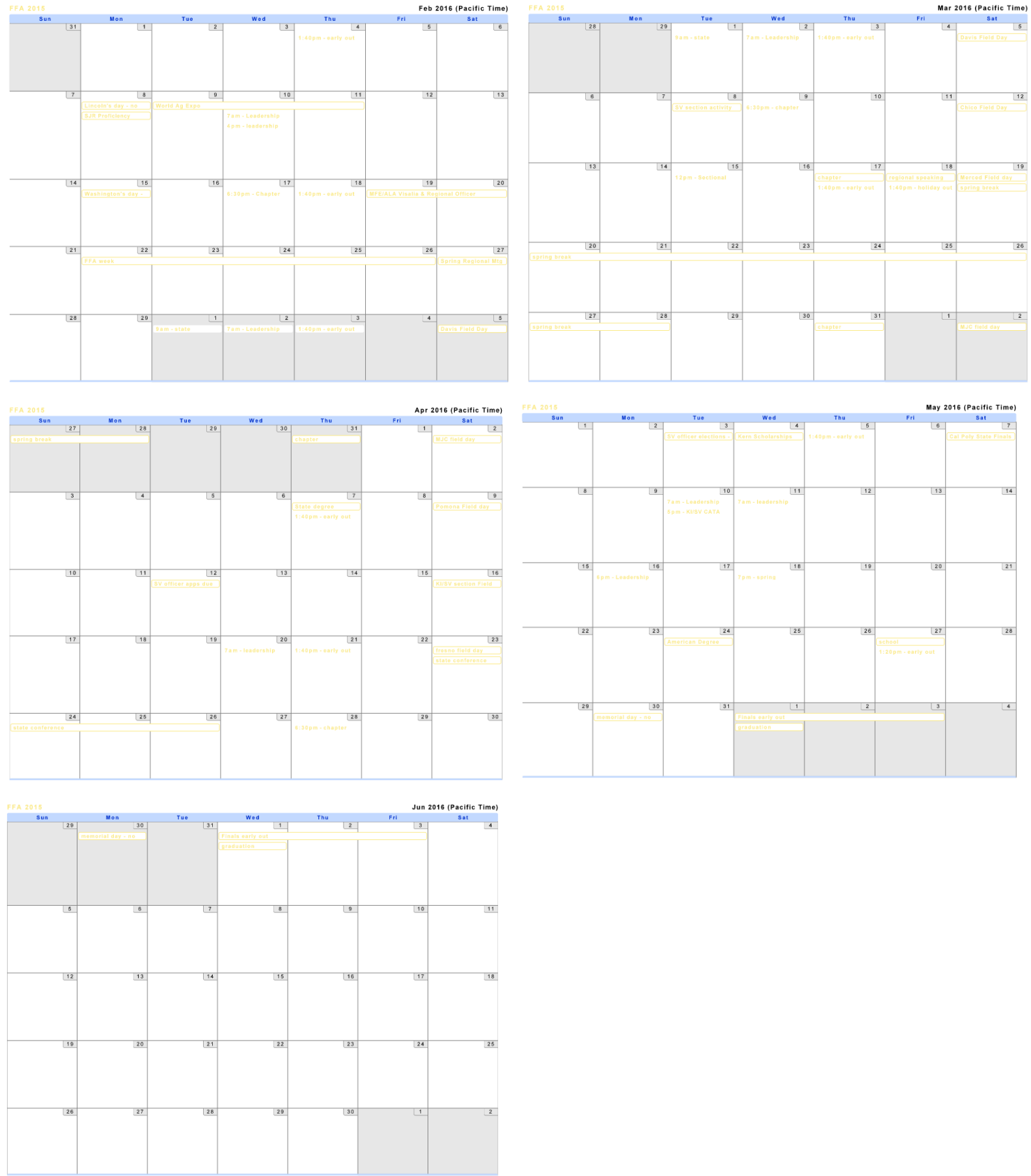

6 | P a g e Ridgeview FFA Program of Activities 


\section{The Aims \& Purposes of the FFA Organization}

The National FFA Organization is dedicated to making a positive difference in the lives of students by developing their potential for premier leadership, personal growth and career success through agricultural education. To accomplish its mission, FFA:

- Develops competent and assertive agricultural leadership.

- Increases awareness of the global and technological importance of agriculture and its contribution to our well-being.

- Strengthens the confidence of agriculture students in themselves and their work.

- Promotes the intelligent choice and establishment of an agricultural career.

- Encourages achievement in supervised agricultural experience programs.

- Encourages wise management of economic, environmental and human resources of the community.

- Develops interpersonal skills in teamwork, communications, human relations and social interaction.

- Builds character and promotes citizenship, volunteerism and patriotism.

- Promotes cooperation and cooperative attitudes among all people.

- Promotes healthy lifestyles.

- Encourages excellence in scholarship

\section{FFA Colors}

In 1929 at the second National FFA Convention held in Kansas City, National Blue and Corn Gold were adopted as the official colors as the Future Farmers of America. National Blue was selected to represent the blue field of our nation's flag while corn gold is used to symbolize the golden fields of ripened corn that unify our country.

\section{FFA Motto}

The FFA motto gives members twelve short words to live by as they experience the opportunities in the organization.

Learning to Do, Doing to Learn, Earning to Live, Living to Serve.

7|Page Ridgeview FFA Program of Activities 


\section{The FFA Emblem}

The national FFA emblem, consisting of five symbols, is representative of the history, goals and future of the organization. As a whole, the emblem covers the broad spectrum of FFA and agriculture. Each element within the emblem has unique significance

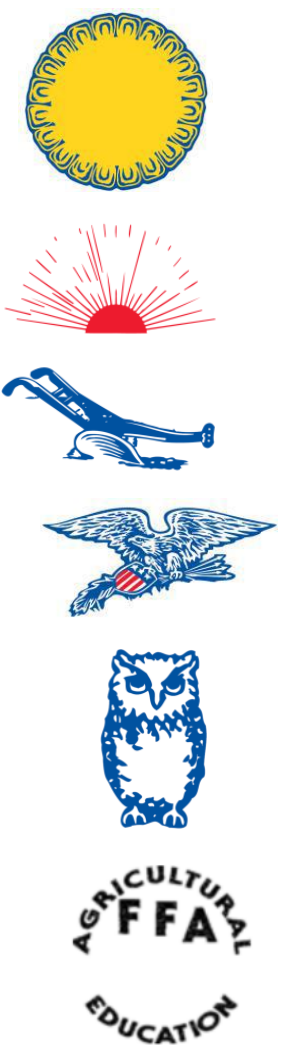

The emblem and the letters "FFA" are protected by trademark registration in the U.S. Patent Office and

by Public Law 105-225, 105th Congress.

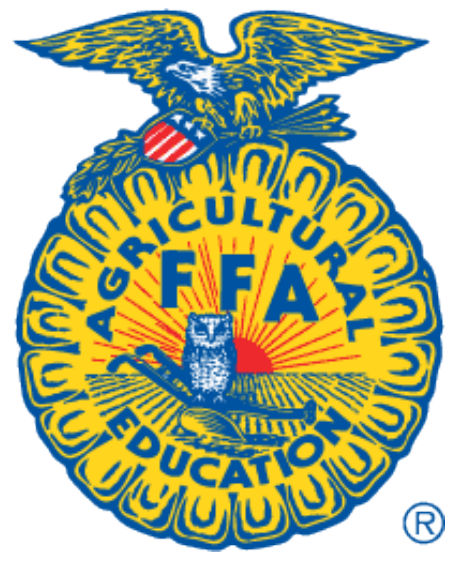

The Cross section of the ear of corn provides the foundation of the emblem just as corn is the foundation of early American agriculture. It is also the symbol of unity, as corn is grown in all 50 states.

The Rising sun signifies progress and holds a promise that tomorrow will bring a new day glowing with opportunity.

The Plow signifies labor and tillage of the soil, the backbone of agriculture and the historic foundation of our country's strength.

The eagle is a national symbol, which serves as a reminder of our freedom and ability to explore new horizons for the future of agriculture.

The owl, long recognized for its wisdom, symbolizes the knowledge required to be successful in the industry of agriculture.

The words "Agricultural Education" and "FFA" signify the combination for learning and leadership necessary for progressive agriculture. 


\section{The FFA Creed}

I believe in the future of agriculture, with a faith born not of words but of deeds - achievements won by the present and past generations of agriculturists; in the promise of better days through better ways, even as the better things we now enjoy have come to us from the struggles of former years.

I believe that to live and work on a good farm, or to be engaged in other agricultural pursuits, is pleasant as well as challenging; for I know the joys and discomforts of agricultural life and hold an inborn fondness for those associations which, even in hours of discouragement, I cannot deny.

I believe in leadership from ourselves and respect from others. I believe in my own ability to work efficiently and think clearly, with such knowledge and skill as I can secure, and in the ability of progressive agriculturists to serve our own and the public interest in producing and marketing the product of our toil.

I believe in less dependence on begging and more power in bargaining; in the life abundant and enough honest wealth to help make it so--for others as well as myself; in less need for charity and more of it when needed; in being happy myself and playing square with those whose happiness depends upon me.

I believe that American agriculture can and will hold true to the best traditions of our national life and that I can exert an influence in my home and community which will stand solid for my part in that inspiring task.

The creed was written by E. M. Tiffany, and adopted at the 3rd National Convention of the FFA. It was revised at the 38th Convention and the 63rd Convention.

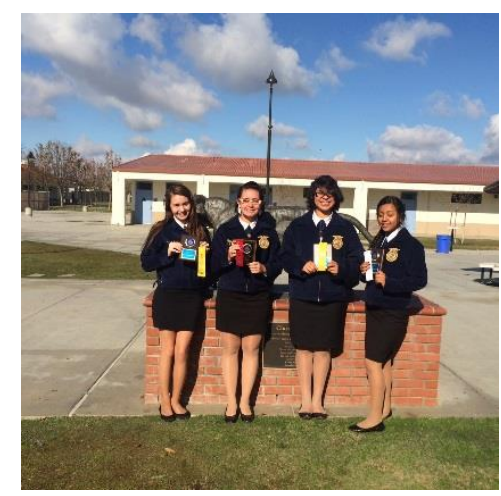

9|Page Ridgeview FFA Program of Activities 


\section{Official FFA Uniform}

\section{Female Official Dress}

- Black skirt. Skirt is to be at least knee length, hemmed evenly across the bottom, with a slit no longer than 2 inches above the knee, excluding the kick pleat. Black slacks may be appropriate for traveling and outdoor activities.

- White collared blouse and official FFA blue scarf.

- Black dress shoes with a closed heel and toe (No boots, sandals, open-toed shoes, or tennis shoes.)

- Natural colored nylon hosiery.

- Official FFA jacket zipped to the top.

\section{Male Official Dress}

- Black dress pants. (No jeans - blue or black, leather, pleather, etc.)

- White dress shirt and official FFA tie

- Black dress shoes with a closed heel and toe. (No boots, sandals, open-toed shoes, tennis shoes.)

- Black socks.

- Official FFA jacket zipped to the top.

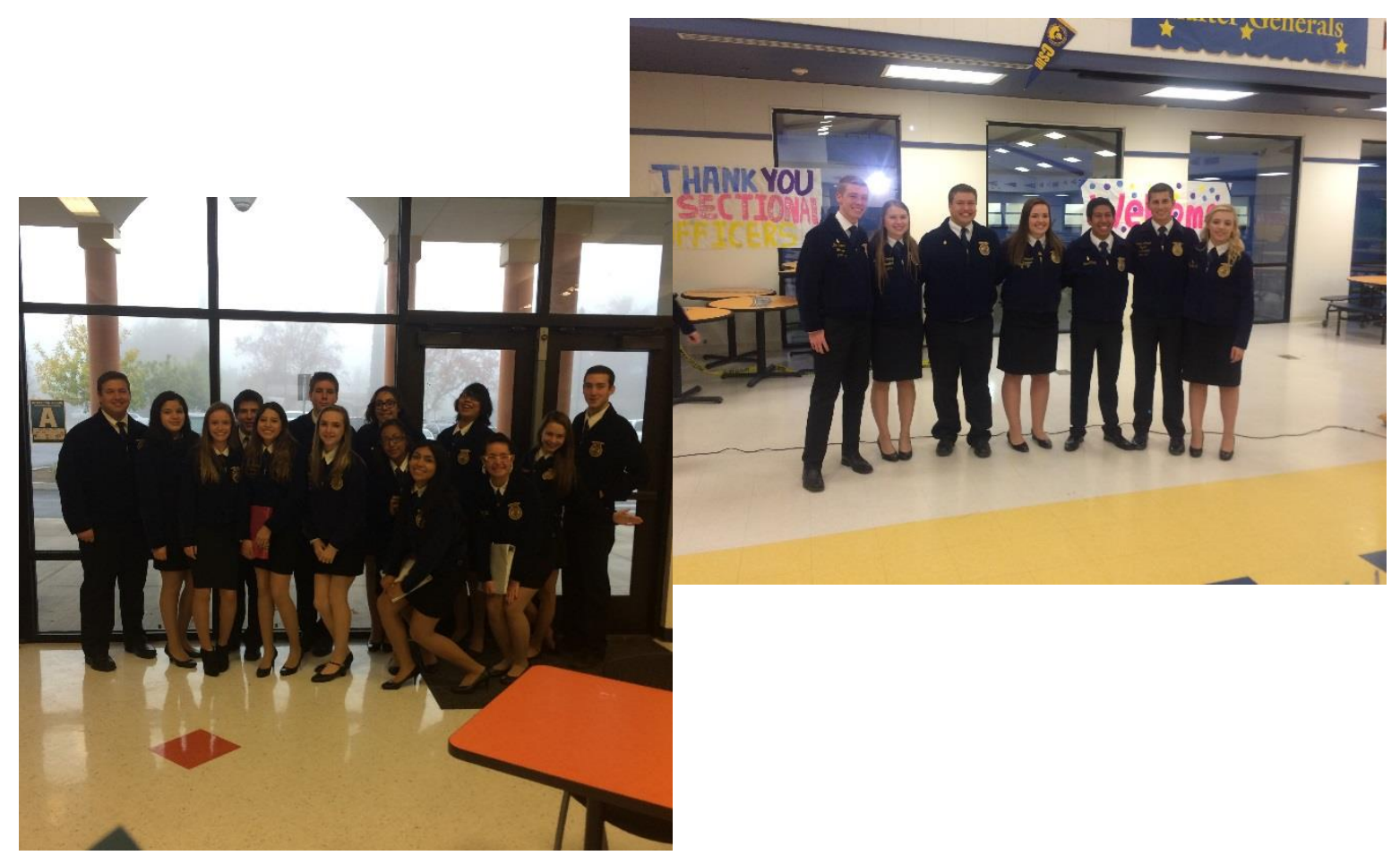




\section{Proper Use of the FFA Jacket}

1. The jacket is to be worn only by members.

2. The jacket should be kept clean and neat.

3. The back of the jacket should have only a large official FFA emblem, the name of the state and the name of the local chapter, region, district or area. The front of the jacket should have only a small official FFA emblem, the name of the individual, one office or honor and the year of that office or honor.

4. The jacket should be worn on official occasions with the zipper fastened to the top. The collar should be turned down and the cuffs buttoned.

5. The jacket should be worn by members and officers on all official FFA occasions, as well as other occasions where the chapter or state association is represented. It may be worn to school and other appropriate places.

6. The jacket should only be worn to places that are appropriate for members to visit.

7. School letters and insignia of other organizations should not be attached to or worn on the jacket.

8. When the jacket becomes faded and worn, it should be discarded or the emblems and lettering removed.

9. The emblems and lettering should be removed if the jacket is given or sold to a non-member.

10. A member should act professionally when wearing the FFA jacket.

11. Members should refrain from use of tobacco and alcohol when underage at all times when representing the FFA. In addition, members should exhibit their leadership qualities when they encounter substances including tobacco and alcohol and serve to discourage others from inappropriate behavior.

12. All chapter degree, officer and award medals should be worn beneath the name on the right side of the jacket, with the exception that a single State FFA Degree charm or American FFA Degree key should be worn above the name or attached to a standard key chain. No more than three medals should be worn on the jacket. These should represent the highest degree earned, the highest office held and the highest award earned by the member. No more than three medals should be worn on the jacket.
11 | P a g e
R i d g e vi e w
FFA Program
of Activities 


\section{FFA Code of Ethics}

People are always observing you. Your actions when you wear the FFA jacket or represent the organization become part of the organization's image. To keep the image of the FFA and members sharp, delegates at the 1952 National FFA Convention adopted a Code of Ethics for FFA members to follow. The FFA Code of Ethics still protects the FFA image. It also guides members to make positive, healthy choices - and not only during FFA activities. The code of ethics guidelines are good to follow during all occasions and functions.

\section{The FFA Code of Ethics}

FFA Members conduct themselves at all times to be a credit to their organization, chapter, school, community and family. I pledge to:

$>$ Develop my potential for premier leadership, personal growth and career success

Make a positive difference in the lives of others.

$>$ Dress neatly and appropriately for the occasion.

$>$ Respect the rights of others and their property.

$>$ Be courteous, honest and fair with others.

$>$ Communicate in an appropriate, purposeful and positive manner.

$>$ Demonstrate good sportsmanship be being modest and winning and generous in defeat.

$>$ Make myself aware of FFA programs and activities and be an active participant.

$>$ Conduct and value a supervised agricultural experience program. 
Strive to establish and enhance my skills through agricultural education in order to enter a successful career.

Appreciate and promote diversity in our organization.

\section{Career Development Events}

All FFA contests are designed to be a natural continuation of the instructional program in agriculture education. The contests help to make classes more interesting and give students an additional reason $f$ or doing their best to develop skills. These skills include:

(1) the technical knowledge gained in the classroom

(2) the ability to make sound judgments

(3) the ability to defend their decisions by giving a convincing set of reasons

(4) to build self-confidence and leadership.

\section{Speaking Contests}

CREED SPEAKING is for FFA members in grades 7, 8, and 9. They must present the FFA Creed from memory and answer questions about its meaning and purpose. The event boosts self-confidence, develops their ability to communicate in a powerful, professional manner.

JOB INTERVIEW participants submit a resume, complete job applications and participate in mock job interviews with a panel of possible employers.

EXTEMPORANEOUS PUBLIC SPEAKING participants are given 30 minutes to deliver a speech on one of three assigned agricultural topics. Students must learn to think on their feet and develop an argument quickly and persuasively.

PREPARED PUBLIC SPEAKING allows students to write and deliver a six- to eight-minute speech about a current agriculture-related topic. The speaking skills gained through this event help students excel in school, community and career settings.

IMPROMPTU SPEAKING was added as an official contest this year. It is designed to better prepare students to think on their feet and showcase their understanding of current agricultural and FFA related issues. Upon drawing a topic or keyword, contestants have 1 minute to present their speech that does not exceed 2 minutes to a panel of judges.

\section{Spring Judging Teams}

VEGETABLE CROPS JUDGING The participants will judge classes of vegetables and take an identification exam.
13 | P a g e
R i d g e vi e w
FFA Program
of Activities 


\section{More Opportunities to Stay Involved}

OPENING AND CLOSING is designed to develop public speaking skills and promote the proper use of the Official FFA Opening and Closing Ceremonies.

AGRICSCIENCE FAIR recognizes students who excel in the application of scientific principles and emerging technologies in their agriculture enterprise.

BEST INFORMED GREENHAND is a written test for ninth grade students based on their knowledge of the FFA.

BANKING is designed to expose students to the agriculture business and finance sectors. Students are tested on their knowledge of the history, purpose and functions of the Farm Credit systems as well as mathematical calculations.

NOVICE RECORDS helps students to understand the importance of maintaining sound financial practices through.

COOPERATIVES has students take a written test to showcase their understanding of agriculture cooperatives system.

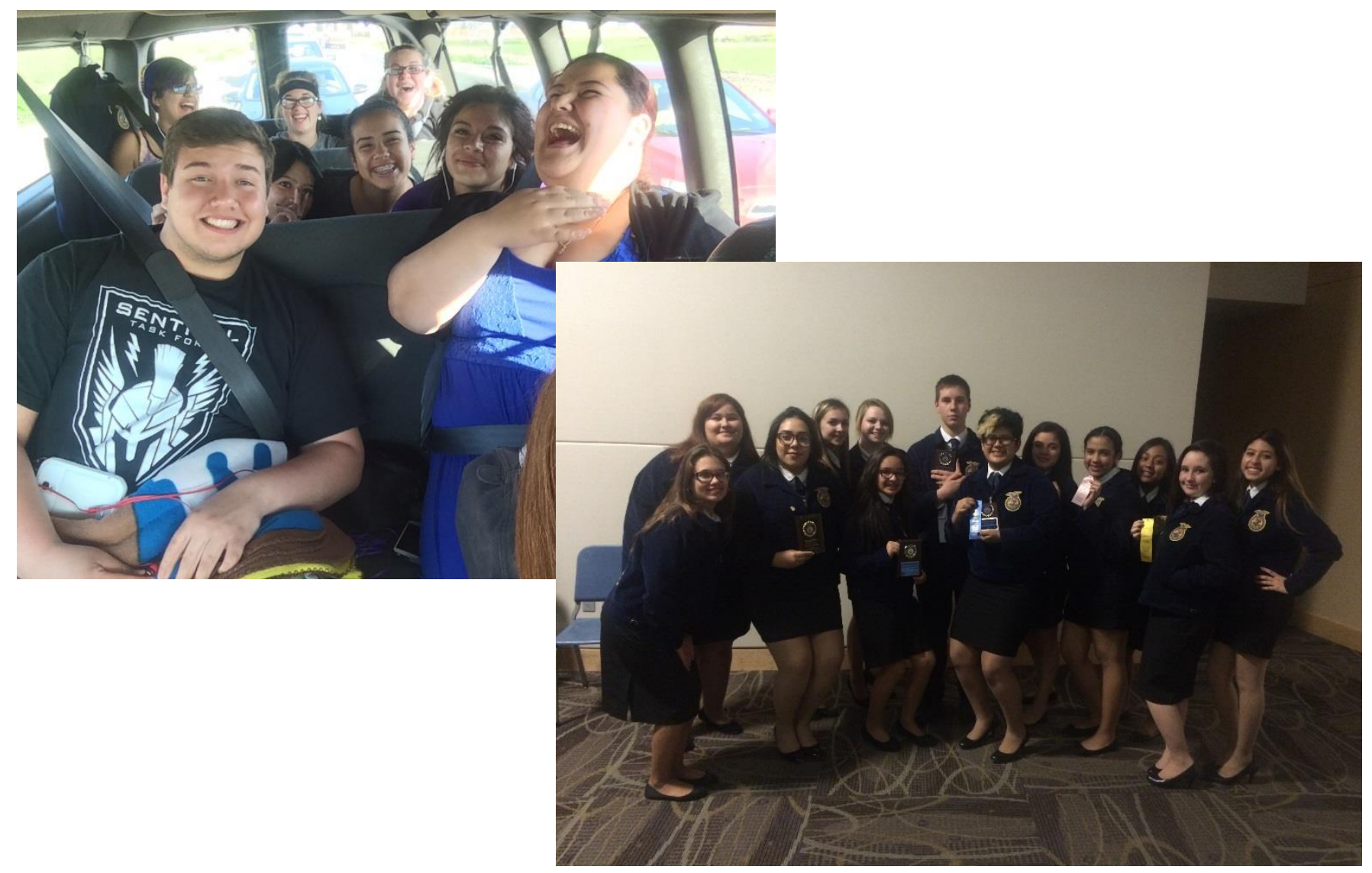




\section{SAE}

What if you could get classroom credit and FFA awards for doing what you like: experimenting with careers, earning money, building a resume and having fun? You can - with a Supervised Agricultural Experience (SAE) program. An SAE is a program you design to gain hands-on experience and develop skills in agricultural career areas that interest you.

You choose an SAE program that lets you discover, explore, experience and excel in careers. In the meantime, you gain skills and experience that pay off in areas of life. Your SAE program can lead you toward personal growth, premier leadership, and career success.

An SAE program is not just another class assignment or graduation requirement. You are truly in charge of your SAE! Although your agriculture teacher will help you learn related information and keep good records, the success or failure of your SAE is up to you. It's an exciting opportunity to prove your abilities to future employers - and to yourself.

\section{Ridgeview FFA SAE Program}

* The Chapter will encourage all members to maintain a Supervised Agriculture Experience (SAE) program.

* The Chapter will encourage members to compete at shows with their SAE.

All Chapter members are expected to work as a team at all fairs and shows

The Chapter will conduct an Exhibitors / Parent evening to inform parents and members of a member's responsibilities.

- The Chapter will require parents of all first time exhibitors to attend a meeting conducted by the Chapter Advisors. This meeting will serve as an informal session to allow parents / exhibitors to become aware of the expectations and responsibilities placed on the Chapter exhibitor.

$>$ All projects exhibited at fairs and shows by members of the Chapter must be entered in the FFA division and only with Advisor approval and supervision.

$>$ Members exhibiting at fairs must maintain academic requirements set forth by Ridgeview High School and the Ridgeview Agriculture Program and FFA. In order to participate in any activity beyond the chapter level, an individual must maintain at least a 2.0 GPA, cannot have more than
15 | P a g e
Ridgeview FFA Program of Activities 
one $\mathrm{F}$, and cannot have received less than a $\mathrm{C}$ grade in any Agriculture class the last eligibility period to the event.

Additional eligibility rule - Students will be given one chance for scholastic ineligibility for showing at fairs. If a student becomes ineligible to show at a fair that they had planned to show, the student will receive a warning. If the same student should become ineligible again to show at a fair that they had planned to show at, the student will no longer be eligible to show with Ridgeview FFA.

- Members are encouraged to apply for local, regional and state proficiency awards.

- Members are encouraged to apply for advanced degrees (i.e. State FFA Degree)

- Members are encouraged to compete in the Local and Sectional Project Competition.

- Members are required to follow project Advisor's recommendations concerning their SAE.

- Members are encouraged to strive to improve and develop their SAE each year.

- Encourage members to develop skills within their SAE through participation and appropriate judging teams.

- Members are encouraged to attend demonstrations, breeding shows, and equipment shows which will enable them to increase their efficiency and knowledge of their SAE.

* Members are encouraged to provide support and help their fellow Chapter members.

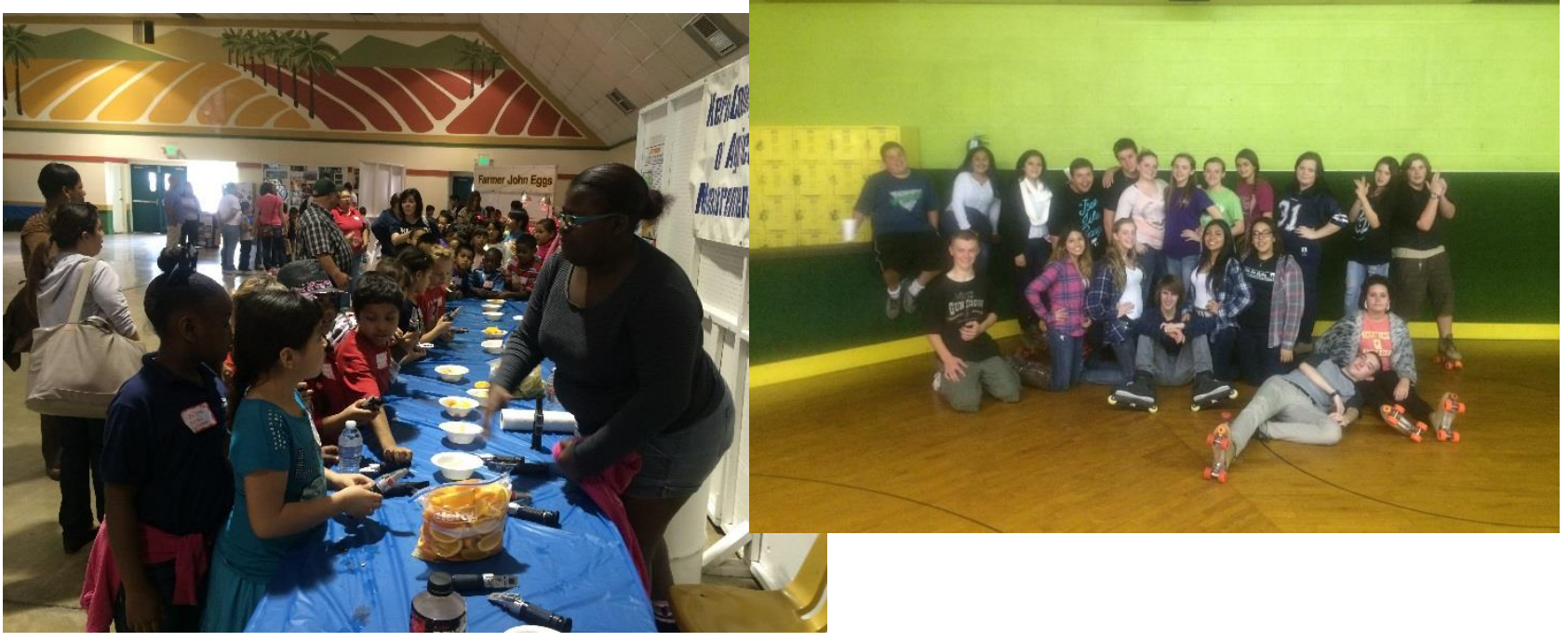

16|Page Ridgeview FFA Program of Activities 


\section{Proficiency Awards}

- The Agricultural Proficiency Awards honor FFA members who, through their Supervised Agriculture Experience projects have developed specialized skills that they can apply toward their future careers.

- Students can compete for awards in 47 areas covering everything from Agricultural Communications to Wildlife Management. Each award area has two categories, placement and entrepreneurship.

- Placement proficiency awards are given to those whose SAEs are related to employment, apprenticeships, or internships at an agribusiness or agriculture-related organization. Entrepreneurship proficiency awards are given to those whose SAEs are related to ownership of an agribusiness or agriculture-related organization.

- Proficiency awards are given out at the local, state and national levels.

\section{Proficiency Award Areas:}

- Agricultural Communications

- Agricultural Education

- Agricultural Mechanics Design and Fabrication

- Agricultural Mechanics Energy Systems

- Agricultural Mechanics Repair and Maintenance

- Agricultural Processing

- Agricultural Sales

- Agricultural Services

- Beef Production

- Dairy Production

- Diversified Agricultural Production

- Diversified Crop Production

- Diversified Horticulture

- Diversified Livestock Production

- Emerging Agricultural Technology

- Environmental Science and Natural Resources Management

- Equine Science

- Fiber and/or Oil Crop Production
- Floriculture

- Food Science and Technology

- Forage Production

- Forest Management and Products

- Fruit Production

- Grain Production

- Home and/or Community Development

- Landscape Management

- Nursery Operations

- Outdoor Recreation

- Poultry Production

- Sheep Production

- Small Animal Production and Care

- Specialty Animal Production

- Specialty Crop Production

- Swine Production

- Turf Grass Management

- Vegetable Production

- Veterinary Medicine

- Wildlife Management 


\section{Leadership}

Leadership is the ability to guide or influence others to work towards a meaningful goal while helping each to develop themselves as group members. Leadership is the ability in a well-adjusted person to handle people, to inspire or influence the actions of others, to make decisions or to move a group to action. Leadership is a contribution to the establishment and attainment of group processes. Therefore, leadership is a quality of group action.

Public Speaking

- $\quad$ Prepared Public Speaking

- $\quad$ Extemporaneous Public Speaking

- Parliamentary Procedure

- Job Interview

- Opening and Closing Ceremonies Speaking Contest

\section{Committees}

- Every member on at least one committee or involved in some kind of activity

- Select Chapter members as Chairpersons for Committees

Degrees and Awards

- Encourages every member to apply for Greenhand and Chapter Farmer FFA Degree Encourage every qualified member to apply for the State and American FFA degree

- Encourage members to apply for State Proficiency Awards

Officer / Leadership Training

- Annual Chapter Retreat for new officers

- Leadership Training Conference for all officers

- Sectional \& Regional Officer Training

- Made for Excellence Leadership Training / Advanced Leadership Academy / Sacramento Leadership / Washington DC Leadership Conference

Meetings

- Conduct meetings in an orderly fashion by utilizing Parli-Pro
$6 \mid \mathrm{P}$ a g e
Ridgeview FFA Program 
- Have regularly scheduled Chapter Officer and Chapter Meetings

- Encourage every member to attend and participate at all meetings

- Send delegates to all Sectional, Regional, State, and National Meetings

\section{Offices}

- Encourage local members to run for local, sectional, regional, and state offices

- Invite Sectional, Regional, and State Officers to speak to your chapter 


\section{Conduct of Meetings}

Hold Regular, Well-Planned Meetings that Capture the Chapter's Interest and Participation

- Have weekly Chapter Officer Meetings

- Have regular month Chapter Meetings

- Call special meetings when necessary

- Conduct regular Executive Meetings in order to maintain solid Chapter communications

- Prepare a well planned program before meetings

- Provide refreshments for Chapter Meetings

- Have frequent and informative committee reports

- Invite parents and the community leaders to the Chapter Meeting

- The duty of the Sentinel is to set the proper paraphernalia out for the Chapter Meeting and to help the President in maintaining order.

- The goal of the Chapter is to have a least 50\% attendance at each Chapter Meeting

- Have an activity after every Chapter Meeting

Special Meetings Should be Held as Necessary

- A special potluck dinner meeting will be held for the Greenhand/Chapter FFA Degree Installation Banquet

- A very special Awards Banquet will be held in May to wrap up the year.

\section{The Official Ceremonies will be used at all Meetings}

- All officers are required to learn their part.

- The officers will wear the official uniform at all meetings

- The necessary paraphernalia will be used at all meetings. 


\section{Scholarships}

Chapter members considering higher education should apply for FFA's numerous scholarships. A variety of companies, individuals, and foundations sponsor the scholarships through the National FFA Foundation and local organizations.

A special application must be completed and sent to the National FFA Center postmarked by February 15 of each year. The application and instructions for the National FFA College and Vocational/Technical School Scholarship Program is available free from the National FFA Website.

\section{Rules and Conditions Governing FFA Trips}

All Ridgeview High School rules are in effect when on FFA functions. Please consult the Parent/Student handbook for questions. The following additional rules apply:

1. Lights out will occur by 10:30pm on all trips.

2. Wake up time will be determined by advisor prior to fair or contest.

3. Students are not to leave the show or contest site without permission of the instructor.

4. All students will stay for the duration of the show or contest and shall show up for all feedings and scheduled barn duties.

5. On all overnight trips students will stay in the areas designated by North FFA.

6. Students will not drive their own vehicles on out-of-town trips. They will need to return to Ridgeview High School by the same means of transportation used to reach the fair/contest, unless approved by the instructor.

7. Failure to follow the above mentioned rules will result in disciplinary action.

8. No student may leave without parents' prior permission without the instructor. Doors will be taped at $11 \mathrm{pm}$ to ensure no students leave their room. 


\section{Fairs}

The Ridgeview FFA Chapter is involved in local fairs throughout the year. Students can exhibit livestock and agricultural still exhibits. The animals that are exhibited include market and breeding sheep, market and breeding beef, market and breeding swine, dairy cattle, rabbits, market, breeding, and dairy goats.

The Fairs that are attended are:

- $\quad$ Kern County Fair

- $\quad$ Desert Empire Fair

\section{ESTIMATED BUDGETS FOR FAIR ANIMALS}

\section{Market Goats}

Ownership period is at least 60 days for market goats. This animal requires time and responsibility, but not as much as a larger animal. You will be responsible for halter breaking, practicing showmanship, and washing the animal. This is a fun project.

\begin{tabular}{|l|l|}
\hline Expenses & \\
\hline Goat & $\$ 250.00$ \\
\hline Feed & $\$ 150.00$ \\
\hline Entry Fees & $\$ 25.00$ \\
\hline Equipment & $\$ 25.00$ \\
\hline TOTAL EXPENSES & $\$ 450.00$ \\
\hline
\end{tabular}

\begin{tabular}{|l|l|}
\hline Income & \\
\hline Sale at Fair (with no buyers set up) & $\begin{array}{l}\text { \$ 500.00 (average price) Can be more with } \\
\text { sponsors }\end{array}$ \\
\hline Profit/Loss & $\mathbf{\$ 5 0 . 0 0}$ \\
\hline
\end{tabular}

\section{Steer}


Ownership period is at least 120 days for a steer. This animal requires a lot of time and responsibility. You will be responsible for halter breaking, practicing showmanship, and washing the animal every other day. You will also need to attempt to grow a winter hair coat in the middle of summer. A "cooling box" is not necessary to do this, but shade and cooling devices, such as misters, are helpful. This is a time consuming, yet fun project.

\begin{tabular}{|l|l|}
\hline Expenses & \\
\hline Steer & $\$ 1000.00$ \\
\hline Feed & $\$ 1000.00$ \\
\hline Entry Fees & $\$ 25.00$ \\
\hline Equipment \& Fitting Supplies & $\$ 50.00$ \\
\hline TOTAL EXPENSES & $\$ 2075.00$ \\
\hline
\end{tabular}

Income

Sale at Fair (with no buyers set up)

Profit/Loss $\$ 2500.00$ (average price) Can be more with sponsors

$\$ 325.00$

Market Sheep

Ownership period is at least 60 days for a market lamb. This animal requires some time and responsibility. You will be responsible for practicing showmanship, exercising, washing, and shearing your animal. This is a time consuming, yet fun project.

\begin{tabular}{|l|l|}
\hline Expenses & \\
\hline Feeder lamb & $\$ 300.00$ \\
\hline Feed & $\$ 200.00$ \\
\hline Entry Fees & $\$ 25.00$ \\
\hline Equipment & $\$ 25.00$ \\
\hline TOTAL EXPENSES & $\$ 550.00$ \\
\hline
\end{tabular}

\begin{tabular}{|l|l|}
\hline Income & \\
\hline Sale at Fair (with no buyers set up) & $\begin{array}{l}\$ 450.00 \text { (average price) Can be more with } \\
\text { sponsors }\end{array}$ \\
\hline Profit/Loss & $\$ \mathbf{- 1 0 0}$ \\
\hline
\end{tabular}

\section{Market Swine}


Ownership period is at least 60 days for a market pig. This animal requires less time than some of the larger animal projects. You will be responsible for training your pig to walk, practicing showmanship, and washing the animal every other day. This is a fun project.

\begin{tabular}{|l|l|}
\hline Expenses & \\
\hline Market Pig & $\$ 300.00$ \\
\hline Feed & $\$ 200.00$ \\
\hline Entry Fees & $\$ 25.00$ \\
\hline Equipment & $\$ 25.00$ \\
\hline TOTAL EXPENSES & $\$ 550.00$ \\
\hline
\end{tabular}

\section{Income}

Sale at Fair (with no buyers set up)

Profit/Loss

$\$ 500.00$ (average price) Can be more with sponsors

$\$-50$

\section{Rabbits (Non-Meat Pen)}

Ownership period is at least 60 days for a rabbit. This animal requires the least amount of time than the other projects, but it does still require a lot of responsibility, just as the other projects do. You will not make money on this project but you will gain a wealth of experience and knowledge on rabbits. You will be responsible for training your rabbit to show, practicing showmanship, and proper nutrition feeding. This is a fun project.

\begin{tabular}{|l|l|}
\hline Expenses & \\
\hline Rabbit & $\$ 60.00$ \\
\hline Feed & $\$ 30.00$ \\
\hline Entry Fees & $\$ 25.00$ \\
\hline Standards of Perfection Book & $\$ 20.00$ \\
\hline Equipment & $\$ 25.00$ \\
\hline TOTAL EXPENSES & $\$ 160.00$ \\
\hline
\end{tabular}

\begin{tabular}{|l|l|}
\hline Income & \\
\hline Potential Premium awards & $\$ 20.00$ \\
\hline Profit/Loss & $\$-\$ 140.00$ \\
\hline
\end{tabular}




\section{Point Award System}

The Point Award system was developed in order to recognize and award those members who are most active in various FFA activities throughout the year. The Top 10 individuals will receive a medal and recognition at our Spring Banquet in May. These members will also be invited to participate in a Top 10 Activity, last year we spent the day at Camelot Park.

2014-2015 Top 10:

1. Kiani Tafulu

2. Benny Gallardo

3. David Stumph

4. Connie Contreras

5. Abigail Ray

6. Cynthia Contreras

7. Allison Burnett

8. Lacey Braun

9. Vicky Gutierrez

10.Leslie Diaz
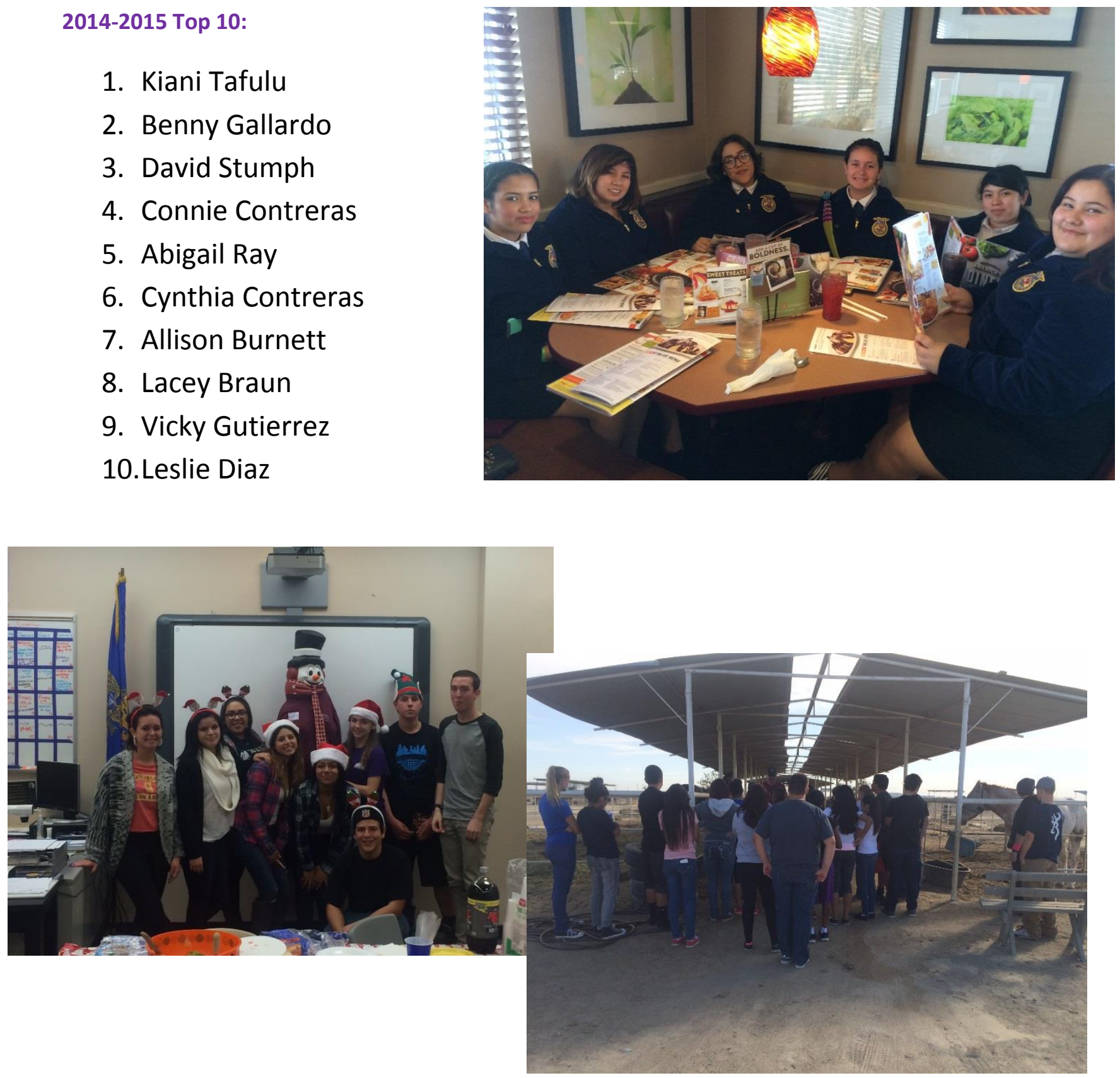


\section{Historical Highlights}

Honorary Chapter Members recognizes individuals from the community that have contributed their time and energy into bettering the agriculture program. This special membership is designed to honor parents, administrators, staff and supporters.

1995- Mr. \& Mrs. Hartline

1995- Mr. \& Mrs. Varney

1996- Mr. Bill Jones

1996- Mr. Roger Riley

1997- Mr. Paul Petite

1997- Mr. Rick Wright

1998- Mrs. Gloria Mundorf

1998- RHS Custodial Staff

1999-2000- Mrs. Diane Ayers

2001- Mrs. Julie Moore

2009- Mr. Bob McConnell

2012- Mr. Mark Hamilton

2013- RHS Information Technology Academy

2014- Ms. Kati Chamberlin

2015- Mr. Ralph Mendes

Past Chapter President The President is stationed by the rising sun as a token of a new era in agriculture. It is under the leadership of the chapter President that North FFA continues to build a legacy for the future of agriculture.

1995-1997 Katie Hartline

1997-1998 Amanda Parks

1998-1999 Dustin Moreno

1999-2000 Matt Brown

2000-2001 Amber Cleaver

2005-2006 Sarah Kaiser

2011-2012 Claudia Culbertson

2012-2013 Claire Culbertson

2013-2015 Connie Contreras

2015-2016 Benito Gallardo 
American Farmer Degree Less than half of one percent of all FFA members are bestowed the American Degree, making it one of the organization's highest honors. This is awarded to students who have demonstrated the highest level of commitment to FFA and their SAE project. Some specifics for degree requirements include leadership, scholastic, community service

1999- Francisco Muro

2000- Amanda Parks

2003- Amber Cleaver

2014- Ann Masengale

State FFA Degree Recipients The State FFA Degree is awarded to FFA members who have demonstrated the highest level of commitment the California State FFA Associations and made significant accomplishments in their Supervised Agricultural Experiences (SAEs).

Approximately 1,800 State FFA Degrees are handed out each year. That number represents approximately $3 \%$ of the California FFA association membership, making it the State's highest honor.

1996-Francisco Muro, Cora Stark, Justin Poncetta, Russell Lamb, Angie Varney, Mindy Phillips 1997- Bryon Johnson, Erin Witten, Katie Hartline, Jason Ross, Craig “Buster" Barnard 1998- Jeanette Stonecipher, Chad Lockard, Paul Carr, Kelly Whatley, Amanda Parks, Kyle Schellenberg, Issac Nelson 2000- Justin Watts, Matt Brown, Callie, Perea, Kasey Torgiani, Brianna Guidera, Kristi Whatley, Tawnie Diebel, Tim Varney 2001- Kyle Blackett, Rodney Bruce, Amber Cleaver, Natalie Crane, Joe crump, Fred Estep, Jared Hixon, Lena Maggi, Steve Muro, Brandi Pixler, Chase Prelipp, P.D. Richardson, John Stalnaker, Amanda Woodard 2002- Laceedon Adame, Krista Breland, Jennifer Rew, Karla Robison

2003- Carla Waters

2004-Randi Bailey, Ashley Bumgarner

2005- Sarah Kaiser

2006- Ashleigh Saulnier

2007- Cassey Bailey, Danielle Frank, Madison Merritt

2008- Brian Foley, Beau Green, Jessica Wheeler

2009- Michael Elcano, Allison Griffith, Alexis Harmening, Jane Masengale, Yvette Singh

2010- Michael Durham, Michelle Grabski, Amber Isbell, Adam Tineo

2011- Claudia Culbertson, Patricia Saulnier, Adam Tineo

2012- Claire Culbertson, Alison Votaw, Ann Masengale

2014- Connie Contreras, Brooke Yoder

2015- Benito Gallardo, Allison Burnett, Victoria Gutierrez, Cynthia Contreras 


\section{Constitution Of The Bakersfield-Ridgeview FFA Chapter}

Written November 17, 1994

Adopted November 30, 1994

Revised August 1, 2011

\section{Article 1. Names and Purposes}

Section 1.01 The name of this organization shall be the "Bakersfield-Ridgeview Chapter of the Future Farmers of America" and the letters "FFA" may be used to designate the chapter, its activities, or members thereof.

Section 1.02 The purposes for which this chapter is formed are as follows:

(a) To develop competent and aggressive agricultural leadership.

(b) To create and nurture a love of agricultural life.

(c) To strengthen the confidence of students of vocational agriculture in themselves and their work.

(d) To create more interest in the intelligent choice of agricultural occupations.

(e) To encourage members in the development of individual occupational experience programs and establishment in agricultural careers.

(f) To encourage members to improve their home and its surroundings, and their community.

(g) To participate in worthy undertakings for the improvement of the agriculture industry.

(h) To develop character, train for useful citizenship and foster patriotism.

(i) To participate in cooperative effort.

(j) To encourage and practice thrift.

(k) To encourage improvement in scholarship.

(I) To provide and encourage the development of organized recreational activities. 


\section{Article II. Organization}

Section 2.01 The Bakersfield-Ridgeview Chapter of FFA is a chartered local unit of the California Association of Future Farmers of America which is chartered $b$ the National FFA Organization.

Section 2.02 This chapter accepts in full the provisions of the constitution and bylaws of the California Association of FFA as well as those of the National FFA Organization.

\section{Article III. Membership}

Section 3.01 Membership in this chapter shall be of three kinds:

(a) Active;

(b) Alumni; and

(c) Honorary, as defined by the National FFA Constitution.

Section 3.02 The regular work of the chapter shall be carried on by the active membership.

Section 3.03 Honorary membership in this chapter shall be limited to the honorary chapter FFA degree.

Section 3.04 Active members in good standing may vote on all business brought before the chapter. An active member shall be considered in good standing when:

(a) They regularly attend local chapter meetings with reasonable regularity.

(b) They show an interest in and take part in the affairs of the chapter.

(c) Are properly affiliated with the state and national FFA organizations.

Section 3.05 Names of applicants for membership shall be filed with the Vice President.

\section{Article IV. Emblems}

Section 4.01 The emblem of the FFA shall be the emblem for the chapter.

Section 4.02 Emblems used by the members shall be designated by the National FFA Organization.

\section{Article V. Membership Degrees and Privileges}

Section 5.01 There shall be four degrees of active membership in this chapter. These degrees are:

(a) The Greenhand FFA Degree

$$
\text { 17|Page Ridgeview FFA Program of Activities }
$$


a. All Greenhands are entitled to wear the regulation bronze pin.

(b) The Chapter FFA Degree

a. All members holding the degree of Chapter FFA are entitled to wear the silver emblem pin.

(c) The State FFA Degree

a. All members holding the State FFA degree are entitled to wear the regulation gold emblem charm.

(d) The American FFA Degree

a. All members holding the American FFA Degree are entitled to wear the regulation gold emblem key.

Section 5.02 Greenhand FFA degree minimum qualifications for election: (Refer to state constitution for a complete list of degree requirements.)

(a) Be regularly enrolled in a class in vocational education for an agricultural career occupation and have satisfactory and acceptable plans for a program of supervise farming and/or other agricultural occupational experiences.

(b) Learn and explain the FFA creed, motto and salute.

(c) Describe the FFA emblem, colors and symbols.

(d) Explain the proper use of the FFA jacket.

(e) Have satisfactory knowledge of the history of the organization.

(f) Know the duties and responsibilities of the FFA members.

(g) Personally own or have access to the official FFA manual.

(h) Submit written application for the degree and for the chapter records.

(i) Must attend Greenhand degree banquet, unless previous permission to be absent from the banquet is approved by the chapter officer team.

Section 5.03 Chapter FFA degree minimum qualifications for election:

(a) Must have the degree of the Greenhand and have a record of satisfactory participation in the activities of the local chapter.

18|Page Ridgeview FFA Program of Activities 
(b) Must have satisfactorily completed at least one year of instruction in vocational agriculture, have in operation an improved supervised farming and/or other agricultural occupational experience program and be regularly enrolled in a vocational agriculture class.

(c) Be familiar with the purposes and program of activities of the state association and the national organization.

(d) Be familiar with the provisions of the constitution of the local chapter.

(e) Be familiar with parliamentary procedure.

(f) Must lead a group discussion for fifteen minutes.

(g) Must have earned by his/her own efforts from his/her supervised farming and/or other agricultural occupations program and deposited in a bank or otherwise productively invested at least $\$ 150.00$ or spent 100 hours on his/her SAE in excess of scheduled class time.

(h) Must attend the Chapter degree banquet, unless previous permission to be absent from the banquet is approved by the chapter officer team.

Section 5.04 State FFA degree minimum qualifications for election:

(a) Qualifications for the State FFA degree are those set forth in the constitution of the state association.

(b) Must go to the state degree banquet.

Section 5.05 American FFA degree minimum qualifications for election:

(a) Qualifications for the American FFA degree are those set forth in the constitution of the National FFA Organization.

Section 5.06 The Advisor shall review the qualifications of members and make recommendations to the chapter concerning degree advancement. 


\section{Article VI. Officers}

Section 6.01 The officers of this chapter shall be as follows: president, vice president, secretary, treasurer, reporter and sentinel. The local advisor shall be the teacher of vocational agriculture in the school where the chapter is located. Officers shall perform the usual duties of their respective offices, as prescribed in the Official FFA Manual.

Section 6.02 Those interested in running for office will fill out and submit an application and interview with the nominating committee chosen by the current year's officer team. The nominating committee will then slate up to 2 candidates per office based on qualification, skill and leadership potential. The new officers will be selected by a ballot of majority vote of the active membership.

Section 6.03 An officer can be removed from office when at least one of the following situations exists:

(a) The officer has lost interest in performing the duties of their office as shown by their officer attendance record maintained by the chapter secretary:

a. An officer will be considered for dismissal when they have obtained three strikes:

i. Officers are required to be at all executive officer team meetings and trainings, all chapter meetings and those events designated by the officer team as mandatory for all officers to attend (this may include the Chapter Officer Leadership Conference, the annual awards banquet, degree ceremonies and etc.).

ii. If an officer will miss a meeting or event as specified above, they must call the President prior to the absence.

iii. Officers present at the meeting where an officer is absent will vote whether the absence is excused or unexcused, with the majority casting the absence category.

iv. Three excused absences constitute one strike.

v. Each unexcused absence constitutes one strike.

(b) An officer's quarter grade in their agriculture class falls below an $80 \% \mathrm{~B}$ and/or their GPA for any one quarter falls below a 2.0.

(c) The remaining officers and/or advisor has noticed a decrease in the ability to perform the job, the positive/influential attitude necessary to perform as a chapter officer, and/or the involvement required of a chapter FFA officer. 
In a closed officer meeting, the highest-ranking officer will entertain a motion to remove the officer from office. A second to the motion must be secured. The officer can only then be removed with a majority vote from the executive team, with the advisor's consent. In the event this occurs, the advisor will confiscate all of the past officer's materials. The Vice President will take on the duties of that office until a replacement is installed into office. In a closed officer meeting, the remaining officers, with the consent of the advisor, will decide to either:

(a) Allow the person who was slated against the removed officer in the previous year's election to fill the office, or

(b) Open applications for a replacement, conduct interviews for all qualified applications, and slate and hold elections for the replacement as per Section 6.02.

Section 6.04 The officers of the chapter shall constitute the Chapter Executive Committee. The executive committee shall have full power to act as necessary for the chapter in accordance with actions taken at chapter meetings and various regulations or bylaws adopted from time to time.

Section 6.05 Honorary members shall not vote on nor shall they hold any office in the chapter except that of advisor.

Section 6.06 Chapter officers must hold the Chapter FFA degree, or the Greenhand FFA degree and be eligible to receive the Chapter FFA degree within six months of officer installation.

\section{Article VII. Meetings}

Section 7.01 Regular chapter meetings shall be held once a month during the school year. Special meetings may be called at any time.

Section 7.02 Standard meeting equipment shall be used at each meeting. All regular meeting shall open and close with the official ceremony. Parliamentary Procedure shall be used in transacting all business at each of the meetings.

Section 7.03 Delegates as specified by the State Constitution shall be elected annually from the active membership to represent the chapter at the State Leadership Conference. Other delegates may be names as necessary in order to have proper representation at various other FFA meetings within the state.

Section 7.04 A majority of the active members listed on the secretary membership roll shall constitute a quorum, and a quorum must be present at any meeting at which business is transacted or a vote taken committing the chapter to any proposal or action.
$21 \mid \mathrm{P}$ a g e
R i d g e vi e w
F F A Program 


\section{Article VIII. Dues}

Section 8.03 State and National dues shall be paid by the advisor when purchasing the State FFA Leadership packets.

\section{Article IX. Amendments}

Section 9.01 This constitution may be amended or changed at any regular chapter meeting by a two-thirds vote of the active members present providing it is not in conflict with the State Association constitution or that of the National FFA Organization.

Section 9.02 Bylaws may be adopted to fit the needs of the chapter at any regular chapter meeting by a two-third vote of the active members present providing such bylaws conflict in no way with the constitution and bylaws of either the State Association or the National Organization. 


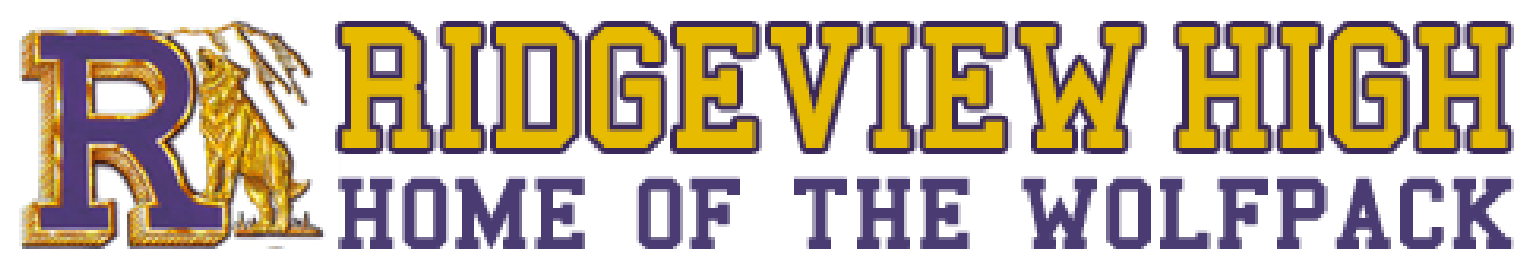

\author{
II \\ Quality Criteria \\ Narratives
}




\section{Quality Criteria Narratives}

\section{Table of Contents}

1. Curriculum \& Instruction (3)

2. Leadership \& Citizenship Development (7)

3. Practical Application of Occupational Skills (9)

4. Qualified \& Competent Personnel (10)

5. Facilities, Equipment \& Materials (12)

6. Community, Business \& Industry Involvement (14)

7. Career Guidance

8. Program Promotion

9. Program Accountability \& Planning

10. Student-Teacher Ratio

11. Full Year Employment

12. Program Achievement 


\section{Quality Criteria 1: Curriculum \& Instruction}

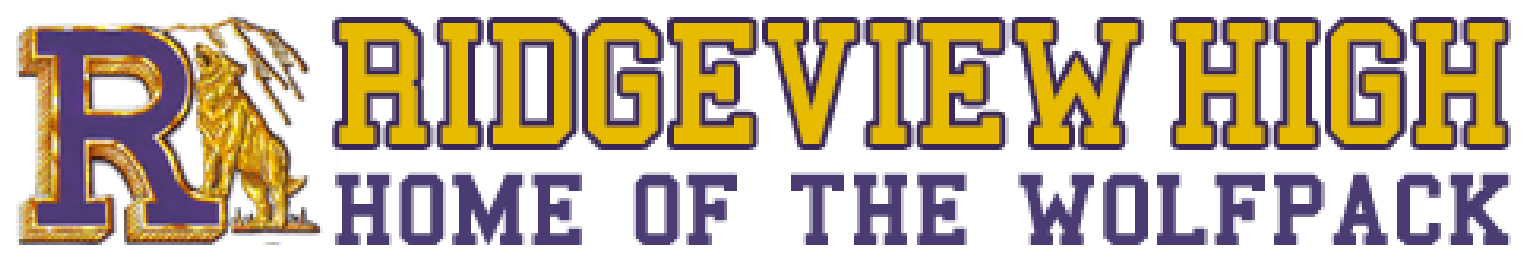




\section{Quality Criteria 1: Curriculum \& Instruction}

1A. The Curriculum includes the components required under Section 52454 of the Education Code: organized classes in the study of agriculture and technology; student supervised agriculture experience; and a program of leadership, organization and personal development.

Currently Ridgeview High School Offers 4 courses to its agriculture students. Students in an agriculture class are automatically enrolled in the FFA. The dues are paid for the students by the chapter. All students are required to have a Supervised Agriculture Experience project as well. The courses offered to students are: Ag Earth Science, Ag Biology, Veterinary Science, and Ag Sales.

Ag Earth Science is a year long course, 10 units, designed for $9^{\text {th }}$ grade students. The class meeets the high school graduation requirement for a physical sciecne and is approved as an UC "G" Elective.

Ag Biology is a year long course, 10 units, designed for $10^{\text {th }}$ grade students. The class meets the high school graduation requirement for a biological science and is approved as an UC “D” Laboratory Science.

Veterinary Science is a year long course, 10 units, designed for $11-12^{\text {th }}$ grade students. The class meets the high school elective graduation requirement and the UC “D” Laboratory Science.

Ag Sales is a year long course, 10 units, designed for $11-12^{\text {th }}$ grade students. The class meets the high school elective graduation requirement.

1B. The Career Technical Education Model curriculum for the Agriculture and Natural Resources Industry Sector are the basis for content of courses offered. Curriculum addresses "Foundation" and "Pathway" standards within the program pathway(s) and course sequences.

Currently, the department doesn't have a definite pathway. The students complete mostly an agri-science education in the agriculture department. In the future I hope to make a more definite pathway for the students to follow to make a more straightforward path within the department. 


\section{C. Career paths in agriculture have been identified and can be found on a chart or diagram in the Program Plan. (Foundation Standard 3.0)}

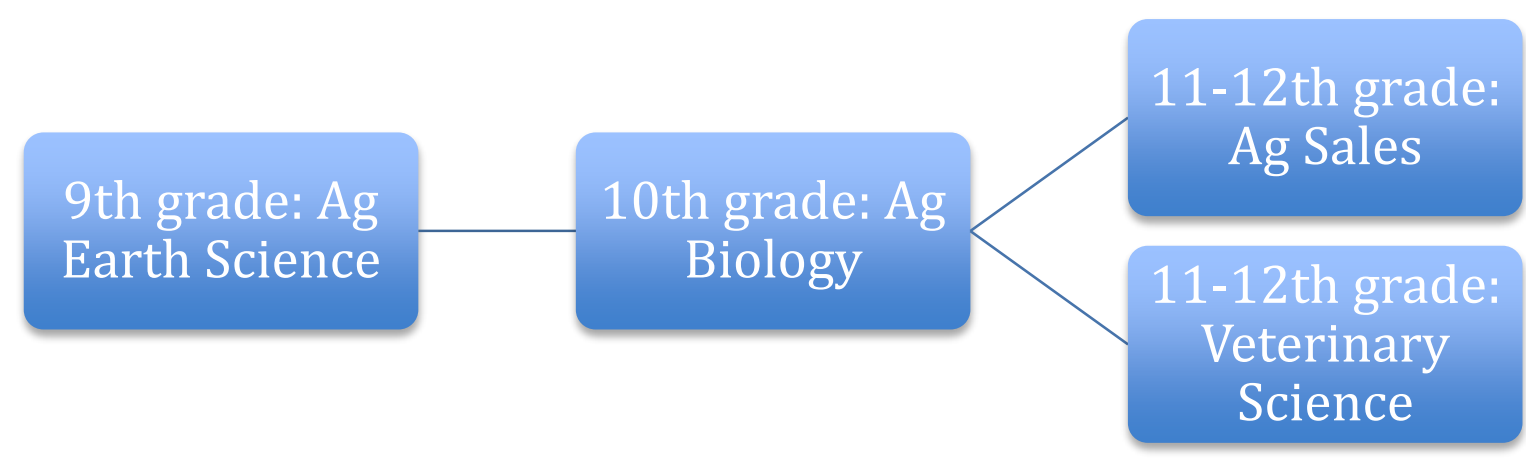

1D. The school master schedule allows students to follow the recommended sequence of agriculture courses to complete the selected career path(s).

The school works the agriculture classes into the master schedule much like they do any class. We also try to accommodate many students with larger class sizes (30-40 students). It would help to offer more than 1 period for the sophomores and for the juniors and seniors. There are some students that struggle getting into an ag class due to conflicts with other courses.

\begin{tabular}{|l|l|l|l|l|l|l|l|}
\hline Teacher & Period 1 & Period 2 & Period 3 & Period 4 & Period 5 & Period 6 & Period 7 \\
\hline $\begin{array}{l}\text { Potterton } \\
2014 / 2015\end{array}$ & Ag Sales & Prep & Ag Bio & Lunch & Ag Earth & Ag Earth & Ag Earth \\
\hline $\begin{array}{l}\text { Potterton } \\
2013 / 2014\end{array}$ & Ag Bio & Prep & Ag Earth & Lunch & Ag Earth & Ag Earth & Vet Science \\
\hline
\end{tabular}

1E. Agriculture Career Awareness information is included in every course. (FS 3.1, 3.2)

All agriculture classes present information on different carrers in the agriculture industry. The focus on each unit is based on the class being taught. Students in the advanced-level courses also create cover letters and resumes to be used in their future. 
1F. The agriculture department untilizes computer hardware and software as an instructional tool. (FS 4.2, 4.6)

The science department (which the agriculture department is housed in) has taken a leading role in technology in our district. When I first started, there were 2 computer labs available on campus and I had 4 computers in my room. Now, I have 10 computers in my classroom. In addition to that, the science department has 5 class sets of chrome books available for me to check out. This has enabled me to convert all students to the iRecord Book and incorporate a lot of technology into my class. I also have a Promethean board and Elmo that I use to assist in instruction. The school has sent our department to Google trainings and conferences and we have hosted district training as well. Incorporating the Chrome Books in the classroom has increased student use of technology and is an important component in the instruction of my classroom.

1G. The agriculture curriculum includes the use of computer aided instruction by utilizing at least one of the following: (FS 4.2, 4.6)

- Computerized Record Book

- Agriculture Term Paper

- Job Resume

- Portfolio Letter of Recommendation
- Agriscience Fair Report

- Agriculture/FFA Speech Manuscript

- Job Cover Letter

- Other Agriculture Related Project

Computers are used often in my classroom. I have moved all of my students onto computerized record books, mostly on the iRecord Book. All resume and cover letters are computerized as well. They also have many projects throughout the year that are completed on the computers. For instance, in the fall, students wrote their SAE Plans in Google Docs and submitted it through Google Classroom.

\section{H. Recordkeeping is taught in the agriculture classes. Every student} maintains and completes (closes out) either an actual SAE Project or Mock Problem. (FS 10.3, 11.0)

First year agriculture students are introduced to record keeping at the beginning of second semester. Then students are expected to keep records of their SAE projects in the iRecord Books afterwards. Occassionaly class periods will be dedicated to students updating their record books, but students can actually update their book from any internet source also.

1I. Record books of all students are maintained in the Department files until one year following graduation.

All the electronic files are kept in the student files that have teacher access for at least one year, if not more. Additionally, backups are kept within the department 
teacher drive. Paper files are kept for the students as well in the cabinets of my classroom.

\section{J. Agriculture courses have been submitted to meet high school graduation requirements and/or University of California a-g credit.}

Since I started at the school, the Ag Earth Science, Ag Biology, and Veterinary

Science courses are all credits for high school science and a-g life science. The Ag Sales class counts for gratuation elective credit. A goal of mine is to change the Ag Sales course into a course that enables students to have a-g credit. 


\section{Quality Criteria 2: Leadership \& Citizenship Development}

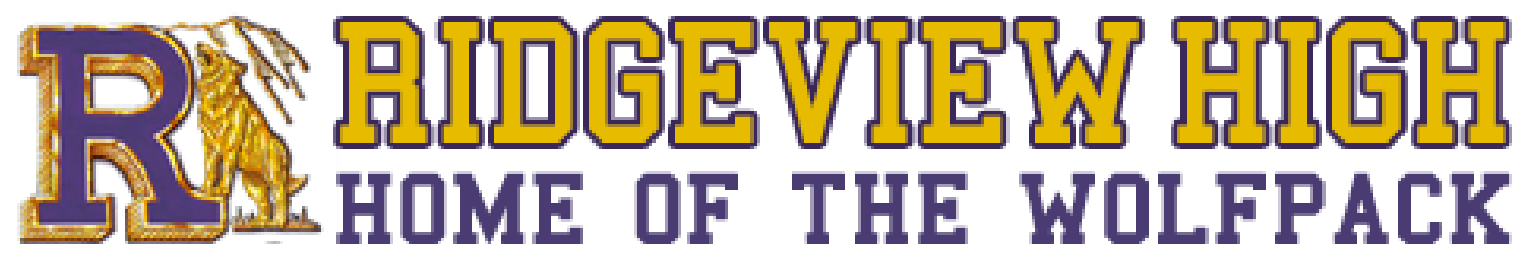




\section{Quality Criteria 2: Leadership \& Citizenship Development}

\section{A. An FFA Chapter has been chartered by the State Association or has been applied for.}

The Ridgeview FFA Chapter was chartered in 1994. The charter is hung on the wall of my classroom.

\section{B. A Chapter Program of Work is developed annually and a copy is furnished to the Regional Supervisor by December $15^{\text {th }}$.}

This is the portion of the quality criteria I have chosen to address for my project. The most recent POW I could find when I was hired was from 2005, so I have been sending Mr. Parker the chapter calendar when the POW is requested.

\section{C. Every student is given a grade based upon participation in leadership activities.}

FFA credit is $5 \%$ of every student's grade. The score is evaluated by the students' earning points towards their FFA score. The students must earn 200 points per semester and the activities are given points based on the work for each activity (chapter meetings are 30 points, contests and conferences are 50 points, etc.).

2D. All students enrolled in agriculture classes are affiliated with the State FFA Association.

All students enrolled in an agricutlure class are on the R2 that is submitted by October $15^{\text {th }}$. 
2E. Based on previous year's records, the department participated in a minimum of 12 activities listed on the FFA Activities Check Sheet.

According to the Annual FFA Chapter Activities Check Sheet our chapter participated in the following activities last year:

1. State Leadership Conference

2. Regional Meeting

3. Greenhand Leadership Conference

4. Opening-Closing Contest-Sectional

5. Best Informed Contest- Sectional

6. Prepared Public Speaking

7. Creed Recitation

8. Job Interview Contest

9. Agricultural CoOp Quiz Contest

10. Submitted State FFA Degree Application

11. FFA Sectional Activity

12. National FFA Week

2F. A minimum of $80 \%$ of the students participate in at least three leadership development activities annually as verified by department records. Activities could include any three of the following intra-curricular activities: (FS 7.0, 9.1, 9.2, 9.3, 9.6, 10.1)

- Local Best Informed Greenhand Contest

- Local Opening \& Closing Contest

- Local Program of Work Committee(s) - Local Demonstration Fair

- Local Agriscience Fair Exhibition

- Local Parliamentary Procedure Contest

- Any Section, Region, or State Activity

- Local Creed Speaking Contest

- Local COOP Quiz Contest

- Local Public Speaking Contest

- Chapter Meeting or Activity

- Other Local Activities

Over $80 \%$ of our members participate in at least three leadership development activities annually. Our documentation consists of a Points of Awards system that I manage. Students are required to attend activities for their grade in my class. The majority of our members attend monthly FFA meetings and other local activities as well. 


\section{Quality Criteria 3: Practical Application of Occupational Skills}

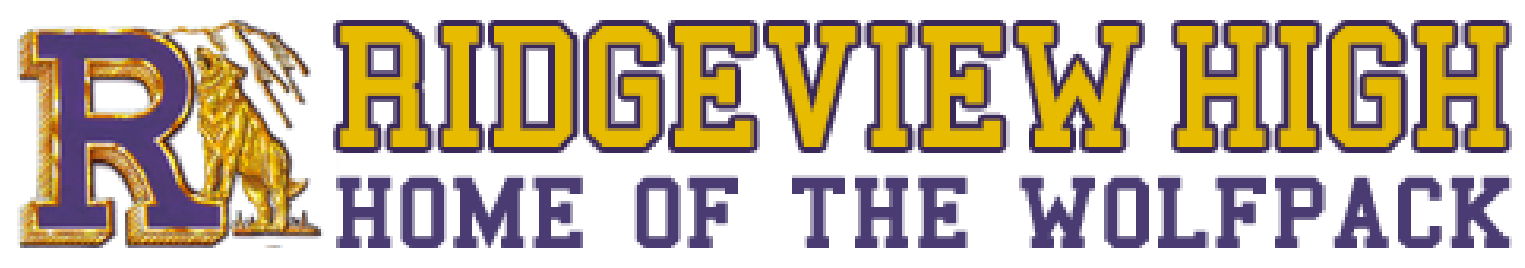




\section{Quality Criteria 3: Practical Application of Occupational Skills}

3A. Student participation in Supervised Agricultural Experience (SAE) is part of the grading criteria for every agriculture student in the program. (FS 10.2)

A student's SAE is $5 \%$ of their grade. In the fall that was evaluated by their plan for their project. This spring I will be evaluating that by the number of hours they have recorded in their books and in their presentation about their project(s). Common projects include fair projects, garden projects, volunteer projects, etc.

3B. First year students have either been engaged in a SAE project(s) or have a plan in place for a SAE, as verified by the Student Data- Career Plan (FS 10.2, 10.3)

First year students are introduced to SAE projects during the fall of their freshman year. They are expected to plan their project by the end of October. They then must start their SAE in order to meet the hours requirement by the spring.

3C. A minimum of $80 \%$ of continuing students are engaged in SAE project(s) as verified by department records. (FS 4.0, 5.0, 6.0, 7.0, 8.0, 9.0, 10.0, 11.0)

The department records we use to verify that $80 \%$ of our students are engaged in an SAE project is their FFA record books. Every student in our department has a record book that is kept is an e-recordbook it is kept on our department harddrive or an iRecord Book that is kept on the California FFA website.

3D. Students with SAE projects are visited by their agriculture teacher at least twice per year as documented by Department records.

SAE visits are documented with a carbon copy project supervision form with one copy given to the student and the other copy being put in the department files. My records show that livestock projects are more frequently than any other SAE project.

3E. A school vehicle is readily available to each agriculture teacher for all SAE activities associated with the program, or each teacehr is adequately compensated for using their own personal vehicle.

I can check out the school truck regularly and always have access to the school trailer. The district provides all the maintenance for the truck and trailer. Also, vans can also be checked out very easily. 


\section{Quality Criteria 4: Qualified \& Competent Personnel}

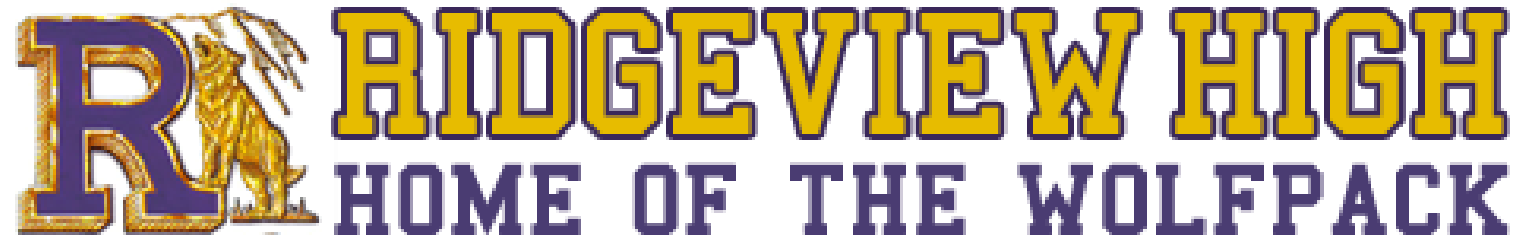




\section{Quality Criteria 4: Qualified \& Competent Personnel}

4A. Every agriculture teacher has the appropriate credential for teaching the subject(s) assigned. Copy of authorizing credential(s) is in the Comprehensive Program Plan.

I hold my single subject credential and specialist instruction credential in agriculture. Additionally, I have supplementary credentials in Biological Sciences, Introductory Science, and Chemistry.

4B. Based on the previous year's records, every agriculture teacehr, teaching at least $1 / 2$ time agriculture, attends a minimum of four professional development activities

I completed the following last year:

- Fall Region Meeting

- Region In-Service

- Section In-Service

- Section In-Service

- Summer Conference

- Kern Farm Bureau Ag Teachers Summer Seminar

- BTSA with an Ag Teacher Support Provider

My long term sub, Kati Chamberlin completed the following last year:

- $\quad$ Spring Region Meeting

- Section In-Service

4C. The agriculture staff meets a minimum of twice a month (This criteria does not apply to single person departments - mark column N/A = Not Applicable)

$\mathrm{N} / \mathrm{A}$

4D. A written record of minutes is kept of action taken during agriculture staff meetings and is kept in Department files or the Comprehensive Program Plan. (This criteria does not apply to single person departments - mark column N/A = Not Applicable)

$\mathrm{N} / \mathrm{A}$ 


\section{E. Teachers are reimbursed for personal expenses they incur while participating in all approved integral activities associated with FFA, SAE, and professional CAT in-service activities.}

On our request to be absent forms we just have to list all personal expenses that we might incur, including food, hotels, conference registration, and mileage on our cars. All expenses come out of our pocket at first and then we have to fill out the reimbursement form and the district will send us a reimbursement check. For hotels we have to provide the invoice and a copy of our bank statement showing the amount we paid. However, we do not need to provide receipts for our meals. If it is supplies for our FFA meetings it is protocol to ask first for petty cash, but if we bring her a receipt and fill out the reimbursement form we will have a check issued to us. 


\section{Quality Criteria 5: Facilities, Equipment, \& Materials}

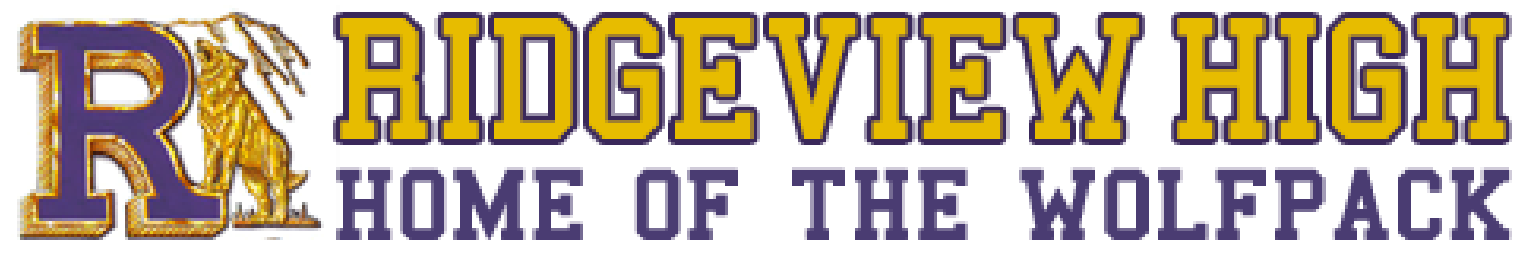




\section{Quality Criteria 5: Facilities, Equipment, \& Materials}

\section{A. Modification of facilities and equipment has occurred when necessary, based on the needs of students, including special departments.}

Over the summer, the school remodeled my new classroom. In the fall I started using my new classroom. It was an important move to get a better lab facility, and to have a lot more room. The classroom is much more functional for the classes I teach. Any major changes that need to be addressed for students needs are completed with the help of the special education department along with the administration.

5B. There is adequate storage space for materials, records, equipment, and supplies.

I have a lot of storage. I have many cabinets and 2 workrooms in my classroom. My classroom was originally a home-economics room, so there are kitchens with plenty of storage. Additionally, I have a c-train and a tool shed for larger equipment.

5C. At least one of the below listed community or school-based laboratory facilities has been provided to accommodate students who have no place for their SAE project(s).

- School Farm Laboratory

- Growing Area
- Greenhouse

- Agriculture Shop

There is a school garden that has a rose bed, citrus trees, tool shed, and 12 raised beds. I have the supplies to make a shade house and plans to complete it soon. There have also been talks to get a greenhouse. Additionally, our district has a farm that is used to house their livestock projects at. I have had talks with administration about getting a farm for the students that is much closer.

\section{D. The Agriculture Department has E-Mail capabilities.}

Our school has email capabilities from all computers on campus. We also have the ability to access our email from our personal computers and cell phones.

5E. The reviewer verifies by visual observation that the agriculture facilities are neat, clean, and orderly.

It is my responsibility to make sure that the agriculture department is kept neat and orderly. The students help in this task. I go through files one to two times per year to get rid of anything we do not use to keep the areas clean and orderly.

5F. Facilities and equipment are regularly maintained, repaired, or replaced. 
Any of our on campus facilities can be repaired or replaced by us filling out a work order request or contacting our grounds manager. Computer issues are maintained by our IT department and our school makes sure our technology is as up to date as possible. For example, I got 10 new computers this summer, and in the fall we got wi-fi and chrome books in the science department. Our truck and trailer are taken to the district maintenance yard for all repairs and maintenance. 


\section{Quality Criteria 6: \\ Community, Business \& Industry Involvement}

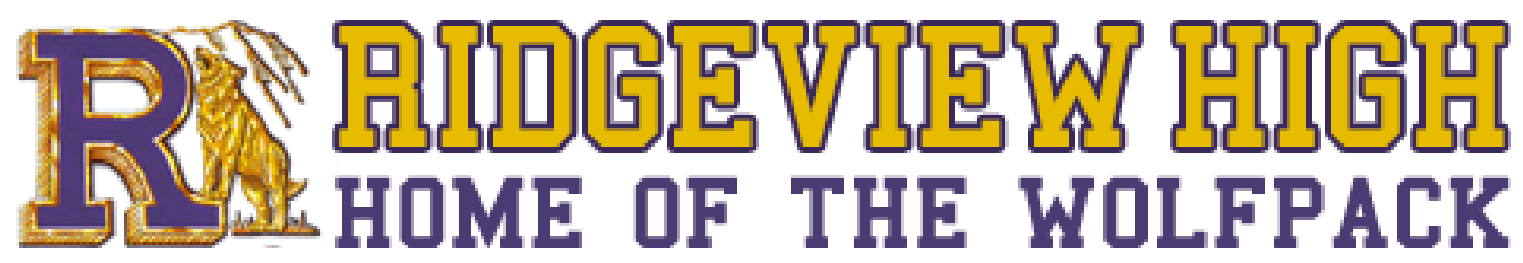




\section{Quality Criteria 6: Community, Business \& Industry Involvement}

\section{A. The Advisory Committee is operational and reflects the committee memberships as outlined in the "Agricultural Education Advisory Committee Manual."}

Our advisory committee consists of the following members:

Ralph Mendes - KHSD Ag Coordinator

Mike Poncetta - Partner-Poncetta Farms Inc.

Kristi Harrer - PCA/Agronomist

Ross Keith - Maintenance/Facility Supervisor at Get Bus

Chris Persons - School Administrator

6B. The Agricultural Advisory Committee meets at least twice each year. (Minutes are available to verify meetings).

The advisory committee meets 2-3 times per year.

\section{Ridgeview FFA Advisory Committee Meeting Minutes 9/3/14}

- Welcome, in attendance: Ross Keith, Ralph Mendes, Michael Poncetta, Jenny Potterton

- 2014 Fair Update: Kern County Fair numbers: 13 pig exhibitors, 15 head of pigs; 2 sheep exhibitors, 3 head of sheep; 2 rabbit exhibitors, 2 rabbits; 3 still exhibit exhibitors with garden-related projects. Target for next year: 5 goats, 2 dairy cattle (talk to Julie Beechinor about financing projects, Mike will help find farmers). Desert empire Fair numbers: 1 sheep

- Garden update: working on getting a shade house, Ralph suggests sterilizing the area to reduce weeds, and putting gravel as flooring. Need to get tables, Ralph will look into a vendor, also could have Matt Riley's students at Mira Monte build tables

- School farm update: gave Ross the insurance, will be working with Chris Persons to get everything worked out so we can use it this summer

- FFA update: Welcome AGtivities had over 70 kids in attendance, 1 American Degree, started a leadership team. Ralph suggests starting a Greenhand officer Team 
- 2014-2015 Planned Calendar: went over planned calendar for the year and how to execute it. Ralph suggests: starting BIG early, will help coach banking, use the 3 greenhands on leadership team as creed competitors, publicize an include administration a community service projects, Ross' Dad $\mathrm{n}$ the board at the homeless shelter

- Advisory Committee structure, etc.: we expect a visit from regional supervisor, Charles Parker in December (4 ${ }^{\text {th}}$ ?), Ross volunteered to be committee chair and will be there to meet with Mr. Parker, review AIG application, need to review checklist next meeting

- Suggested Committee Members: Leticia Davidson (veterinarian), and Dr. Pipkin (Panama equine hospital), Andrea/Tom Price (Bugni's owners, Mike will work on contacting them), Rebecca Bullier (Grimway, 699-2384)

- Questions, Comments, Suggestions: work with administration to get a second teacher

- Next meeting: Nov. 5, 6pm at Molly J's

\section{Ridgeview FFA Advisory Committee Meeting Minutes}

\section{$11 / 5 / 14$}

- Welcome, in attendance Ross Keith and Jenny Potterton

- 2014 Fair Results: Reserve champion AOB lamb, good placings in the hog barn, still exhibits judges choice award and champion vegetable. Already have increased interest for next year, have some students doing cattle projects

- Garden update: shade house Is here, applied for PG\&E grant for gravel, weed cloth, tables, an planting supplies

- Working on a grant for chromebooks for the ag department, AIG specialized grant. Ross's mom may be able to help with the application

- School farm update: haven't heard anything from the administration on what there is left to do for approval, Ross is going to talk to Mr. Persons about it

- FFA update: opening and closing is coming up, planning on taking 6 teams. Public speakers: 3 prepared, 3 extemp, 3 job interview, 2 creed, 1 
impromptu. Ross offered to help with job interview, his mom may be able to proof read the speeches.

- On Site Ag Incentive Grant Review: the on-site review is on December 4 at $1 \mathrm{pm}$. Ross will be in attendance. Went over the AIG checklist. We meet most criteria except 10 and 11. Plan to schedule a meeting between Ross, Ralph Mendes, and Jenny before the review to make sure we know what to expect. Plan out what we need money for.

- Suggested Committee Members: continue trying to recruit the people mentioned at the last meeting

- Questions, Comments, Suggestions: Spoke about how to get better meeting attendance: maybe do an online meeting forum, don't plan dates so far in advance, suggest 3 dates and let the committee members choose

6C. The Agricultural Advisory Committee has assisted in the development or revision of the following components of the Comprehensive Program Plan, as evidenced in the Ag. Advisory Committee minutes

- Job Market Description

- Total Program Goals \& Objectives

- Course Subject Matter Outlines

- 5 Year Facility \& Equipment Acquisition

- Targeted Occupations

- Program Description Courses, SAE, FFA

- Program Completion Standards

- Current Year Budget

- Graduate Follow Up

- List of Active placement Sites

The Advisory Committee has assisted in the development and/or revision of the course of study, program goals and objectives, and program calendar.

6D. The contact information of the Advisory Committee Chair has been provided on the cover of this checklist.

Our current Advisory Chair is Ross Keith. 


\section{Quality Criteria 7: Career Guidance}

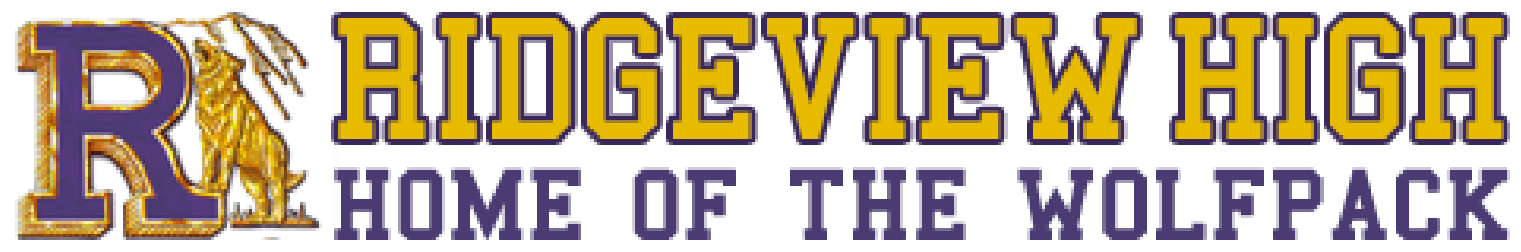




\section{Quality Criteria 7: Career Guidance}

7A. Students are counseled regarding (FS 3.0)

- Career opportunities in Agriculture and Agribusiness

- Agriculture and academic courses necessary to complete career pathway offerings

- Post-secondary education and training options

Career opportunities are presented to each class in the agriculture department. Whenever we go to a college for a contest we always try to take a tour so students can see what is available to them. I also put college information into FFA trips. For instance, I make sure all the kids go to the college building at the World Ag Expo in Tulare, and tour the campuses at Colleges when we have competitions.

7B. All students have a completed career plan (Student Data Sheet) and it is updated annually. (F.S. 3.3)

Students complete a student data sheet every year prior to the October $15^{\text {th }}$ deadline.

7C. Efforts have been made, or completed, to articulate with Community Colleges and/or Universities (ie., 2+2+2 articulation agreements).

Our counseling staff works closely with the local community college to complete articulation agreements with Bakersfield College. 


\section{Quality Criteria 8: Program Promotion}

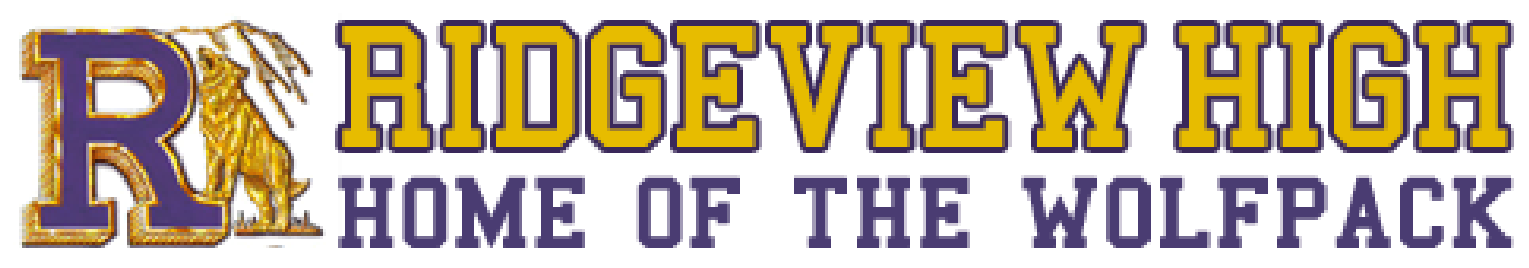




\section{Quality Criteria 8: Program Promotion}

\section{A. An Agriculture Education program recruitment brochure or similar document is used to promote the program.}

A traditional brochure is used for program recruitment. Also, we have a department Instagram to promote the program as well. Larger pictures and awards are used to promote the program and draw interest, particularly at $8^{\text {th }}$ grade recruitment.

\section{B. Students have alternative means of overcoming financial barriers to participate in program activities. (Includes FFA, SAE, Leadership Activities.)}

All students are allowed to take out a loan with local banks for all SAE projects. They are responsible for obtaining their loan and paying it off at the end of their project. For all FFA activities with a cost we give students enough advanced notice that they are able to fundraise to pay for their trip, students are also able to work off parts of their expenses by working in the ag department. I can also supply students with items to start garden projects at their own house.

\section{C. The Agriculture Department conducts recruitment activities with local feeder school.}

Our school has a freshman recruitment night. The agriculture department is featured at the event that enables students to see their high school opportunities. This also enables them to ask questions about the department to teachers and students alike. 


\section{Quality Criteria 9: Program Accountability \& Planning}

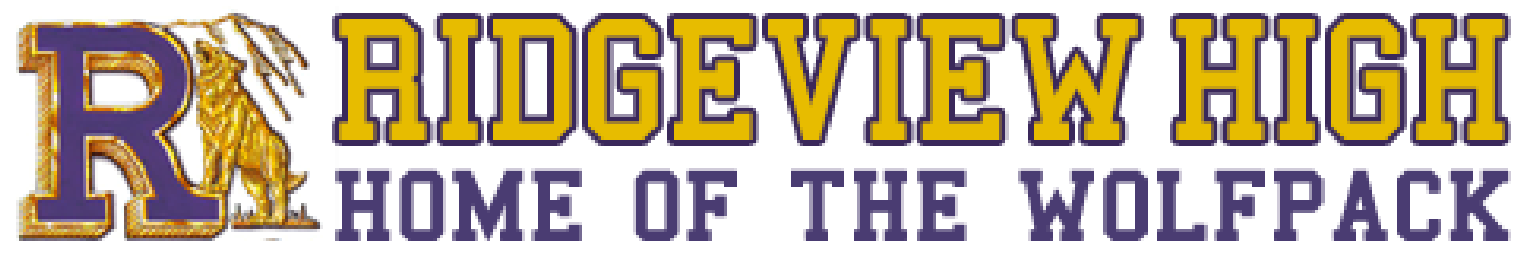




\section{Quality Criteria 9: Program Accountability \& Planning}

9A. A Comprehensive Program Plan is on file with the Regional Supervisor and a copy is retained in the local department files.

Mr. Parker has a Program Plan on file for the Ridgeview FFA program.

9B. Updates of the Program Plan are sent to the Regional Supervisor by November $15^{\text {th }}$. These updates include: (1) Five Year Equipment Acquisition Schedule; (2) Chart of Staff Responsiblities; (3) FFA Program of Work; (4) Advisory Committee Roster; and (5) Advisory Committee Minutes.

9C. A follow-up system is used which gathers the following information from program

- Status of employment or school enrolled within

- Opinion regarding the value and relevance of the agriculture program

- Suggestions for improving the agriculture program

We ask students at the end of their Seniro year what plans they have for the next year. As part of the survey we also ask what their favorite part of the agriculture program was and what we could do to improve the program. So far our senior classes have been small that it has been easy to keep track of students and their future plans. As the department grows, I will have to be more proactive with tracking the graduates.

9D. The Graduate Follow Up data collected was entered with the On-line R2/FFA Roster Data Entry by October $15^{\text {th }}$.

Our graduate follow up data was entered by the October $15^{\text {th }}$ deadline.

9E. The Agriculture Department analyzes their student retention numbers each year and develops strategies to help increase retention within the program.

With it being my $2^{\text {nd }}$ year at the school, I am able to analyze the retention a lot better. I have seen greatly increased retention, with a junior/senior class that had about 20 kids my first year, now having about 40 kids this year. In the future, I do hope to offer more courses for the students to reduce the amount of conflicts with courses like advanced chior and strong body for football. Each year retention is improving. 


\section{Quality Criteria 10: \\ Student-Teacher Ratio}

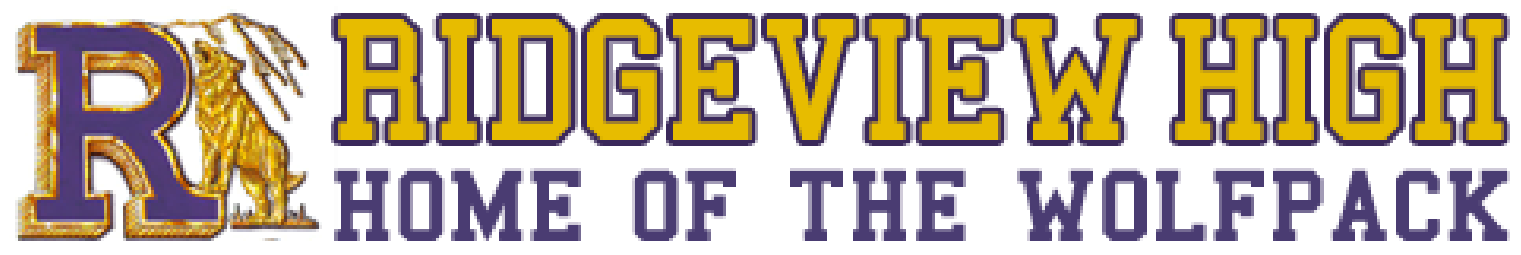




\section{Quality Criteria 10: Student-Teacher Ratio}

10A. Shop and laboratory-based classes have no more than 20 students enrolled, Classroom-based classes have no more than 25 students enrolled.

I don't meet classroom size. I have 30-40 students in each class.

10B. The total number of students enrolled in agriculture classes does not exceed 75 students per teacher. First year students enrolled in agriculture courses will be counted as .5 for purpose of determining the total count only.

I do not meet classroom size. I have about 175 total students. 


\section{Quality Criteria 11: Full Time Employment}

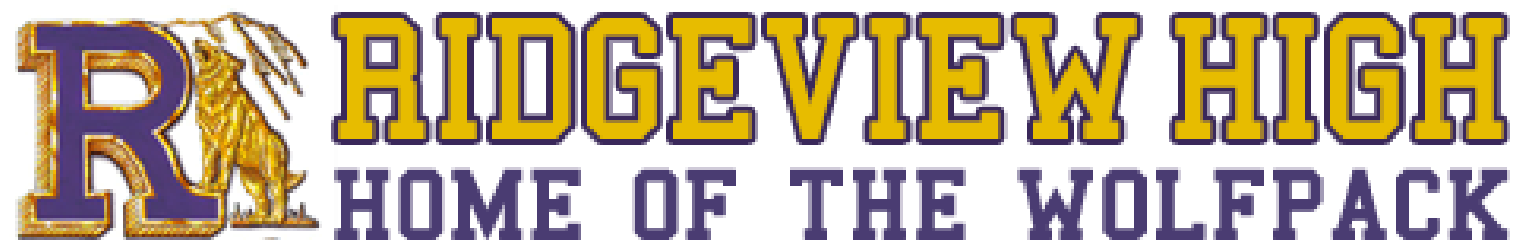




\section{Quality Criteria 11: Full Time Employment}

11A. A full-time teacher is employed year-round for each 75 students enrolled in the agriculture program and is compensated no less than $\$ 2,000$.

Each teacher in the district is given an extra 36 days of pay in the summer to supervise SAE projects.

11B. During the school year, one teaching period for Supervision is assigned to each agriculture teacher. This project supervision period is in addition to the preparation period normally assigned to all teachers in the school. This requirement may also be met if a period is not available by financially compensating the agriculture teacher(s) at the equivalent cost of providing one period for supervision.

I am not compensated for project supervision. 


\section{Quality Criteria 12: \\ Program Achievement}

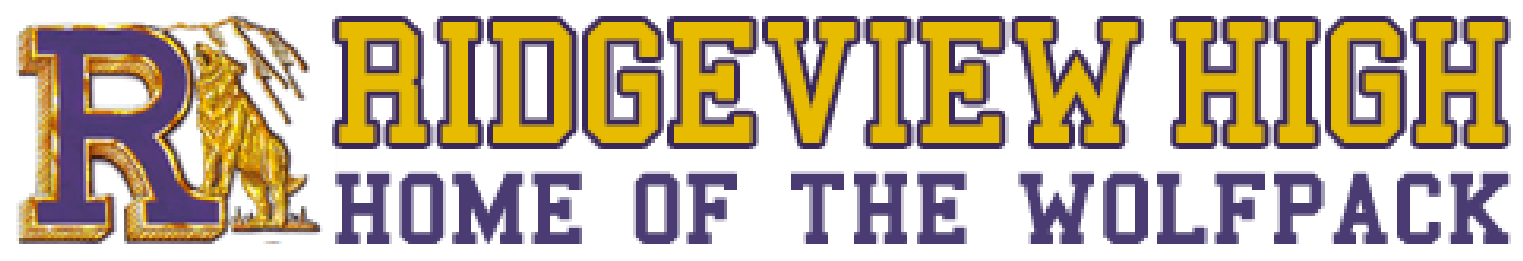




\section{Quality Criteria 12: Program Achievement}

12A. The agriculture Program meets the requirement of Program Achievement

I did not meet Quality Criteria 12 due to State Degree numbers. 


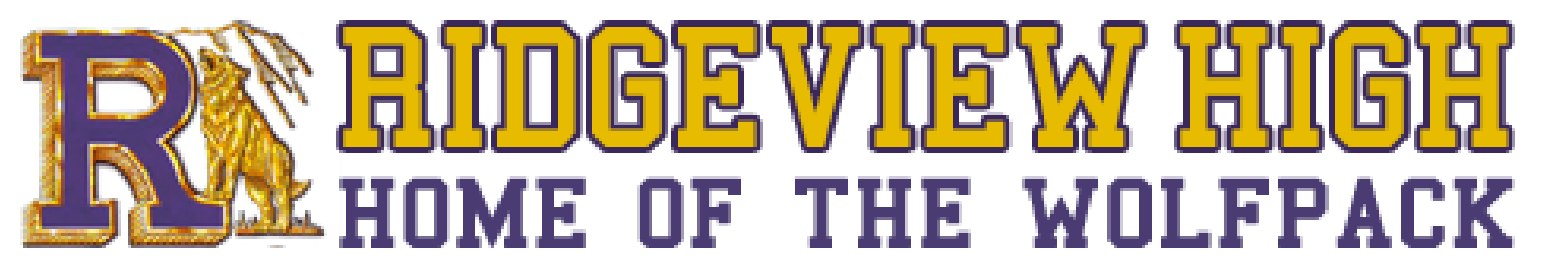

\section{III}

Supporting

Completion

Materials 


\section{Supporting Completion Materials}

\section{Table of Contents}

A. Student Data Sheets

B. Permanent Vo-Ag Student Records

C. Course Outlines

D. Daily Grade Sheets

E. SAE Supervision Form

F. Wall Chart of SAE Visits

G. SAE School Board Approved Policy Statement

H. Program of Work

I. FFA School Board Approved Policy Statement

J. Agriculture Department Recruitment

K. FFA Chapter Scrapbook

L. Summer Activities Calendar

M. Graduate Follow-Up Survey

N. Graduate Status

O. Vo-Ag Comprehensive Plan

P. Advisory Committee Agendas \& Minutes

Q. Student Program Plan

R. Proficiency Standards

S. Teaching Credentials

T. Department Calendar

U. Daily Logs

V. Professional Growth Activities

W. R2 Report

X. Extended Contract Rationale

Y. Completed Travel Plan

Z. CATA Membership Card

AA. Reports Submitted to Administration

BB. Wish List 
CC. Agriculture Department Operating Budget \& Synopsis

DD. Duties \& Responsibilities Synopsis

EE. Chart of Responsibilities

FF. Substitute Teacher Procedures and Plans

GG. Program Completers

HH. Dual Enrollment

II. Department Reimbursements 
Supporting Completion

Materials A: Student Data Sheets

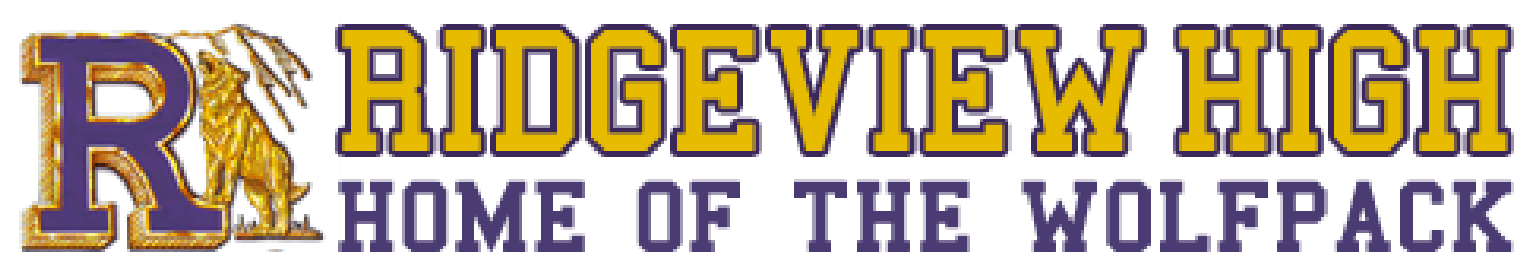




\section{A. Student Data Sheets}

Student Data Sheets are filled out at the beginning of each school year, and per recommendation from Mr. Parker, they are used as enrollment instruction for current students in the Spring. They are stored in the student files in my classroom.

Supporting Completion Material Attached 

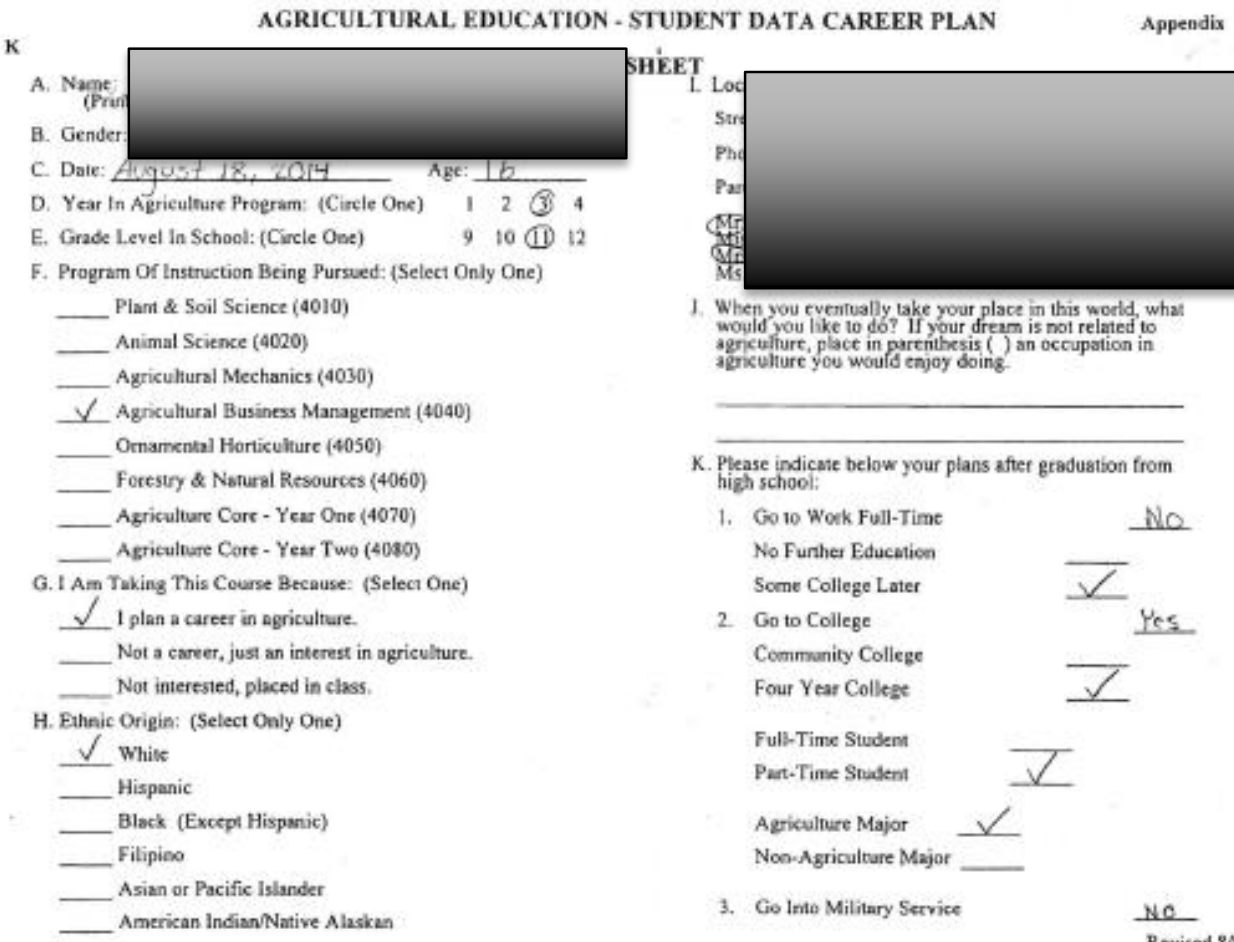

K. Please indicate below your plans after graduation from
high school:

1. Go 10 Work Full-Time No Furher Education Some College Later

2. Go to College Community College Four Year College Full-Time Studen Part-Time Student Agriculture Major Nos-Agriculture Major

3. Go Inte Military Service

\section{STUDENT PROGRAM PLANNING FORM}

L. Planned course of study so meet occupational goal. By scheol year, list all classes pervieusly taken, currently being taken, and planned to be taken in

the future.

FRESHMAN YEAR

$(20) \cdot 13)$

SOPHOMORE YEAR

$(20) 3=\mathrm{M})$

INIOR YEAR

$(20)-15)$

SENIOR YEAR

\begin{tabular}{|c|c|c|c|c|c|c|c|c|c|c|c|}
\hline ans & & & & & & $20 \mathrm{H}-1$ & & & $(20) 5-16)$ & & \\
\hline & Traber & 20 & 2008 & Hectaen & $s$ & cur & renous & 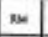 & cus & twones & m \\
\hline Farth Srien. & - Mertz & & Biology & $M 5 . P$ & & $A G$ Buishests & $M=P$ & 107 & Ag Vet Scion & ce Msp & \\
\hline & & & & & & Diama & Mus, haseluse & & & & \\
\hline & & & & & & Chemistry & Mr. Yoon & & & & \\
\hline & & & & & & English II & Mr. Staver & & & & \\
\hline & & & & & & Spornish 1 i & Mis Duthel & & & & \\
\hline & & & & & & Adv. Algebra & Mr.Smith & & & & \\
\hline & & & & & & & & & & & \\
\hline
\end{tabular}

M. Supervised Practical Experience Plan (Project program should be relsted to career goal).

\begin{tabular}{|c|c|c|c|c|c|c|c|}
\hline sos & 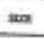 & soc & $\operatorname{six}$ & 302 & $\operatorname{ma}$ & soc & ma \\
\hline Garden & 1 & Morket Animel & 1 & Market Animal & 1 & & \\
\hline & & & & & & & \\
\hline & & & & & & & \\
\hline & & & & & & & \\
\hline
\end{tabular}

N. Planned Departmental Aetivities (FFA)

\begin{tabular}{|l|l|l|l|}
\hline Chapter Mectings & & & \\
\hline & & & \\
\hline & & & \\
\hline & & & \\
\hline & & & \\
\hline
\end{tabular}




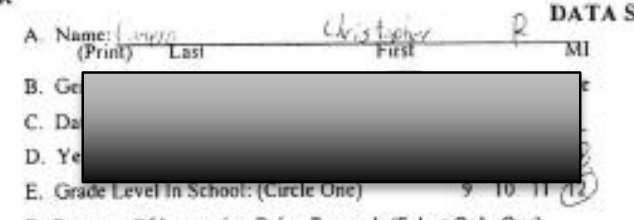

F. Program of Inseruction Being Pursued (Select Only One) Plant \& Soil Science (4010)

Animal Science (4020)

Agricultural Mechanics (4030)

$X$ Agricultaral Business Management (4040)

Omamental Herticulture (4050)

Forestry \& Natural Resources (4060)

Agriculture Core - Year One (4070)

Agricullure Core - Year Two (4080)

G. 1 Am Taking This Course Because: (Select One) I plan a career in agriculture.

Not a career, just a interest in agriculture. Not interested, placed in class.

H. Ethnic Origin: (Select Only One)

White

Haspanic

B_ack (Except Hispanic)

Filipino

_ Asian or Pacific Islander

American Indian/Native Alaskan

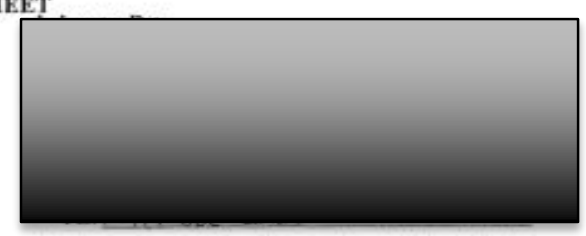

1. When you eventually take your place in this world, what agriculture, place in parenthesis ( $)$ an occupaticn in serieulture you would enjay doing.

Gexis atobuer (

K. Please indicale below your plans after graduation from high school:

1. Go to Work Full-Time No Further Edoxation Some Coliege Later

2. Go 10 Coliege Community College Four Year College

Full-Time Student Part-Time Student

Agriculture Major Non-Agriculture Msjor $\longrightarrow$

3. Go Into Military Service

annjob and torf 10 ilfipld Tevised 8103

STUDENT PROGRAM PLANNING FORM

L. Planned course of study to meet eecupational goal. By achool year, tist all classes previously taken, currensly being taken, and planned to be taken in

the future.

FRESHMAN YEAR $(2011-\pi)$

SOPHOMORE YFAR

\begin{tabular}{|c|c|c|c|c|c|c|c|c|c|c|c|}
\hline \multicolumn{3}{|c|}{$(201-\pi)$} & \multicolumn{3}{|c|}{$\left(20^{2}-1\right)$} & \multicolumn{2}{|c|}{$\begin{array}{l}\text { JUNIOR YEAR } \\
(2012-10)\end{array}$} & \multicolumn{4}{|c|}{$\begin{array}{l}\text { SENIOR YEAR } \\
(201 \%-5)\end{array}$} \\
\hline auss & nuom & $\omega$ & ous & meas & $=0$ & moss & rescoss & $\infty$ & ans & retase & sas \\
\hline og vartis & nertz & & $456 \ldots$ & areste & & $v_{\text {est }}$ & fortator & & $4,28 v$ & atacien & \\
\hline & & & & & & & & & & & \\
\hline & & & & & & & & & & & \\
\hline & & & & & & & & & & & \\
\hline & & & & & & & & & & & \\
\hline & & & & & & & & & & & \\
\hline & & & & & & & & & & & \\
\hline
\end{tabular}

M. Supervised Practical Experience Plan (Project program should be related to career goal).

\begin{tabular}{|c|c|c|c|c|c|c|c|}
\hline $\tan$ & $2 x$ & ser & ane & $\operatorname{ses}$ & $s z x$ & sos & sne \\
\hline Groyen & & Garden & & SZute was & & Shots an 2 ay & \\
\hline & & & & & & & \\
\hline & & & & & & & \\
\hline & & & & & & & \\
\hline
\end{tabular}

\begin{tabular}{|l|l|l|l|}
\hline & & & \\
\hline & & & \\
\hline & & & \\
\hline & & & \\
\hline & & & \\
\hline
\end{tabular}


K

A.

B.

C.

D. Year In Agriculture Program: (Circle One)

E. Grade Level In School: (Circle One) 9 (10) 1112

F. Program Of Instruction Being Pursued: (Select Only One) Plant \& Sail Science (4010)

Animal Seience (4020)

Agricultural Mectanics (4030)

Agricultural Business Masagement (4040)

Ormamental Horticalture (4050)

Forestry a Narural Resources (4060)

Agriculture Core - Year One (4070)

Agricultuen Core - Year Two (4080)

G. I Am Taking This Course Because: (Seleet Ose)

1 plan a career in agriculture.

$\checkmark$ Nol a careet, just an interest in agriculture.

Not inierested, placed in class.

H. Ethnix Origin: (Select Only One)

$\checkmark$ White

Hispanic

Black (Except Hispanic)

Filipino

Asian or Pacific Islander

American Indian/Native Alaskm

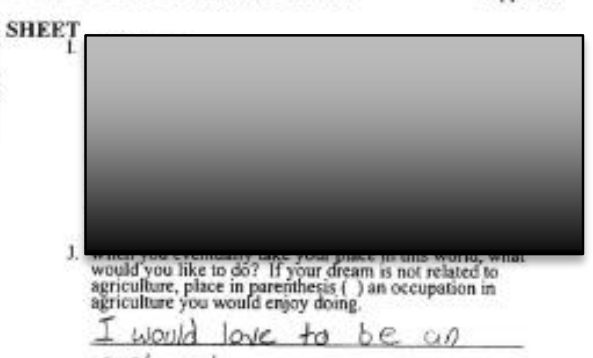

I would lovic to be an esst. vet.

K. Please indicate below your plans after graduation from
high sehool:

1. Go 10 Work Full-Time No Further Edacation Some College Later

2. Go to College Commusity College

Four Year College

Full-Time Studen

Part-Time Student

Agriculture Major

Non-Agriculture Major

3. Go Into Military Service

Revised $8 / 03$

STUDENT PROGRAM PLANNING FORM

L. Planned course of study to meet occupational goal. By school year, list all classes previously taken, currently being taken, and planned to be taken in

the future.

\begin{tabular}{|c|c|c|c|c|c|c|c|c|c|c|c|}
\hline \multicolumn{2}{|c|}{$\begin{array}{l}\text { FRESHMAN YEAR } \\
(20)\end{array}$} & \multicolumn{3}{|c|}{$\begin{array}{l}\text { SOPHOMORE YEAR } \\
(2014-15\end{array}$} & \multicolumn{3}{|c|}{$\begin{array}{l}\text { RUNIOR YEAR } \\
(20+1)\end{array}$} & \multicolumn{4}{|c|}{ SENIOR YEAR } \\
\hline ouss & mease & 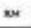 & ouss & mons & $\approx$ & oust & moom & su & aser & munes. & m \\
\hline & & & History & Reison & & vet $5 c$ en & & & & & \\
\hline & & & English & Lames & & Englishil & & & & & \\
\hline & & & Biabgysy & Pottalion & & Adv Algehas & & & & & \\
\hline & & & Lunch & & & & & & & & \\
\hline & & & Spaish I & Dutweel & & & & & & & \\
\hline & & & Geametry & Stair & & & & & & & \\
\hline & & & $\mathrm{Pe}$ & Tuckes & & & & & & & \\
\hline
\end{tabular}

M. Supervised Practical Experience Plan (Project program should be related to career goal).

\begin{tabular}{|c|c|c|c|c|c|c|c|}
\hline $2 a x$ & $\operatorname{sex}$ & - & In & 208 & sa & 206 & sat \\
\hline & & & & & & & \\
\hline & & & & & & & \\
\hline & & & & & & & \\
\hline & & & & & & & \\
\hline
\end{tabular}

N. Planned Departmental Activities (FFA)

\begin{tabular}{|l|l|l|l|}
\hline & & & \\
\hline & & & \\
\hline & & & \\
\hline & & & \\
\hline & & & \\
\hline
\end{tabular}

Parencs/Guardians Signature: 
K
A. $\operatorname{Nam}_{\mathrm{P}}$

B. Gend

C. Date

D. Year In Agriculture Program: (Circle One)

E. Grade Level In School: (Circle One) Plans \& Soin Science $(4010)$ Animal Science (4020)

Agricuhural Mechenics (4030)

Agricultural Business Management (4040)

_Omanental Hertieulure (40SO)

Forestry \& Natural Resoures (4060)

Agriculture Core - Year One (4070)

Agriculture Core - Year Two (4080)

G. I Am Taking This Course Becnuse: (Select One)

Y. 1 plas a carcer in agriculture.

Not a career, just an interest in agriculture.

Not interested, placed in class.

H. Ethnic Origin: (Select Oaly Ore)

White

Y Hispanis

Btack (Except Hispanic)

Filipine

Asian or Pacific Islander

American Indian/Native Alaskan
SHEET

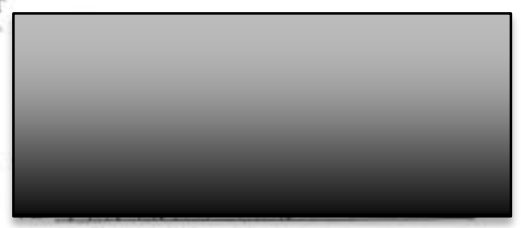

1. When you evectually take your place is this world, what agreulure, place in parenthesis ( ) an occuppatica in sgriculture you would enjoy doing. vet

K. Please indicate below your plans after graduation from

1. Ge so Work Full-Time

No Further Education

Some College Lsiet

2. Go to Collegt

Communisy College

Four Year Colliege

Full-Time Student

Part-Time Student

Agriculture Major

Non-Agriculture Major

3. Go Into Military Service

Revised 8/03

STUDENT PROGRAM PLANNING FORM

L. Plannod course of study to meet occupational gosL. By school year, list all classes previously taken, currenaly being ukiken, and planned to be taken in

the forure

\begin{tabular}{|c|c|c|c|c|c|c|c|c|c|c|c|}
\hline \multicolumn{2}{|c|}{$\begin{array}{c}\text { FRESHMAN YEAR } \\
(203-14)\end{array}$} & \multicolumn{4}{|c|}{$\begin{array}{l}\text { SOPHOMORE YEAR } \\
(201--5)\end{array}$} & \multicolumn{2}{|c|}{$\begin{array}{l}\text { UNIOR YEAR } \\
(2015.45\end{array}$} & \multicolumn{4}{|c|}{$\begin{array}{l}\text { SENIOR YEAR } \\
(204-17)\end{array}$} \\
\hline a.st & reacon & 24 & oxs & machen & (2) & ensen & reveras & $n$ & cuss & menoses & $=$ \\
\hline Dis lefin & Putilay & & an howar & 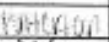 & & Vet SIV & 4if 16162 & & & & \\
\hline & & & spanihse & M100 & & & & & & & \\
\hline & & & Hivley & Difiry & & & & & & & \\
\hline & & & thaish & Lomos & & & & & & & \\
\hline & & & Alsibra 2 & smilh & & & & & & & \\
\hline & & & Wropuly a & 1 smith & & & & & & & \\
\hline & & & & & & & & & & & \\
\hline
\end{tabular}

M. Supervised Practical Experience Plan (Project program should be related to carcer goal).

\begin{tabular}{|c|c|c|c|c|c|c|c|}
\hline tor. & $\mathrm{mox}$ & $\operatorname{son}$ & $\cos$ & ser & sta & sas & $\operatorname{sex}$ \\
\hline Pribits & 1 & Fonl & 1 & & & & \\
\hline & & brids rabbit & & & & & \\
\hline & & & & & & & \\
\hline & & & & & & & \\
\hline
\end{tabular}

N. Planned Departmental Activities (FFA)

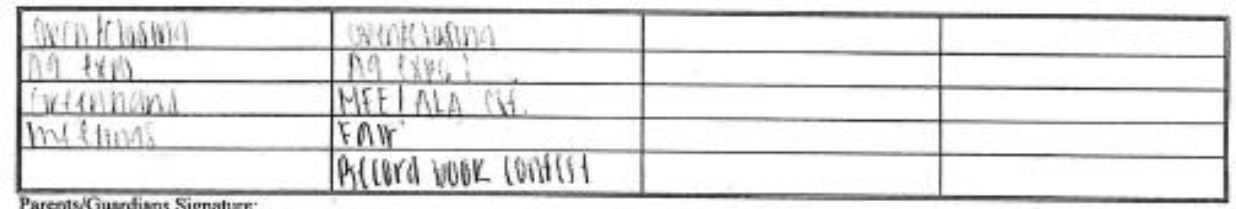


B.

D. Year In Agriculture Program: (Circle One) 1 (2) 344

E. Grade Level In Scheol: (Circle One) 9 (10)11 12

F. Program Of Instruction Being Pursued: (Select Only One) Plant \& Soil Science $(4010)$

Animal Science (4020)

Agricultural Mechanics (4030)

Agricultural Business Management (4040)

Omamental Horticulture (4050)

Forestry \& Natural Resources (4060)

Agriculture Core - Year One (4070)

Agriculture Core - Year Two (4080)

G. I Am Taking This Course Because: (Select One)

I plan a carcer in agriculture.

Not a career, just an isnerest in agriculture. Not interested, placed in class.

H. Ethnic Origin: (Select Only One)

$\checkmark$ White

Hispanic

Black (Except Hispanic)

Filipino

Asian of Pacific Islander

American Indian/Native Alaskan
DENT DATA CAREER PLAN

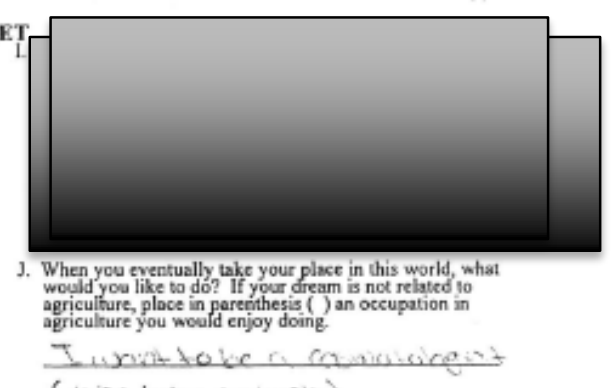

K. Please indicate below your plans affer graduation from

1. Go to Work Full-Time No Further Education Some College Later

2. Go to College Community College Four Year College

Full-Time Student Par-Time Student

Agriculture Major

Non-Agriculture Major $\square$

3. Go Into Military Service

STUDENT PROGRAM PLANNING FORM

L. Planned course of study to meet occupational goal. By school year, list all classes previously taken, eurrenily being taken, and planned to be taken in

the future.

\begin{tabular}{|c|c|c|c|c|c|c|c|c|c|c|c|}
\hline \multicolumn{2}{|c|}{$\begin{array}{c}\text { FRESHMAN YEAR } \\
(20 \text { ) }\end{array}$} & \multicolumn{3}{|c|}{$\begin{array}{c}\text { SOPHOMORE YEAR } \\
(20 \mathrm{k}-15)\end{array}$} & \multicolumn{3}{|c|}{$\begin{array}{l}\text { JNIOR YEAR } \\
(20)\end{array}$} & \multicolumn{4}{|c|}{$\begin{array}{l}\text { SENIOR YEAR } \\
(20 \quad-)\end{array}$} \\
\hline ares & revere & m & soss & manex & $\mathrm{su}$ & coses & Trose & kes & aven & Thacise & $=4$ \\
\hline ge resith & 2 cheldon & & 20 bicares & Achiteula & & Veriscions & Polvelo & & & & \\
\hline & & & Genglish 10 & Kimbie & & Sromalsur & & & & & \\
\hline & & & squenise 2 & Gunetic & & & & & & & \\
\hline & & & Alegbral & wiven & & & & & & & \\
\hline & & & Wrand hiotores & dons & & & & & & & \\
\hline & & & Pe & Truker & & & & & & & \\
\hline & & & & & & & & & & & \\
\hline
\end{tabular}

M. Supervised Practical Experience Plan (Project program should be related to career goal).

\begin{tabular}{|c|c|c|c|c|c|c|c|}
\hline sos & $=$ & $\operatorname{sen}$ & $\sin$ & soc & $=\pi$ & 202 & $\mathrm{mox}$ \\
\hline deut tut $5 x$ & & gavelowing & & & & & \\
\hline & & & & & & & \\
\hline & & & & & & & \\
\hline & & & & & & & \\
\hline
\end{tabular}

N. Planned Departmental Activities (FFA)

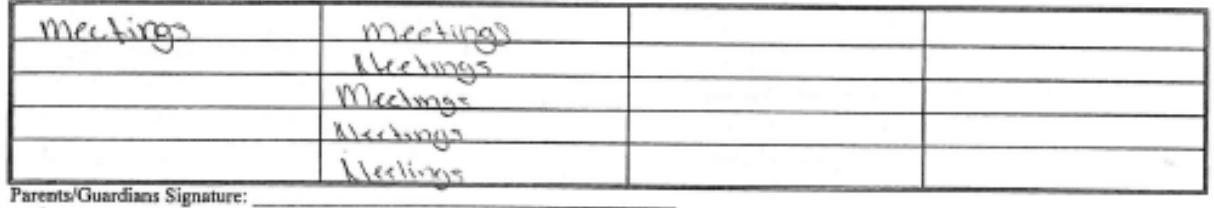


к

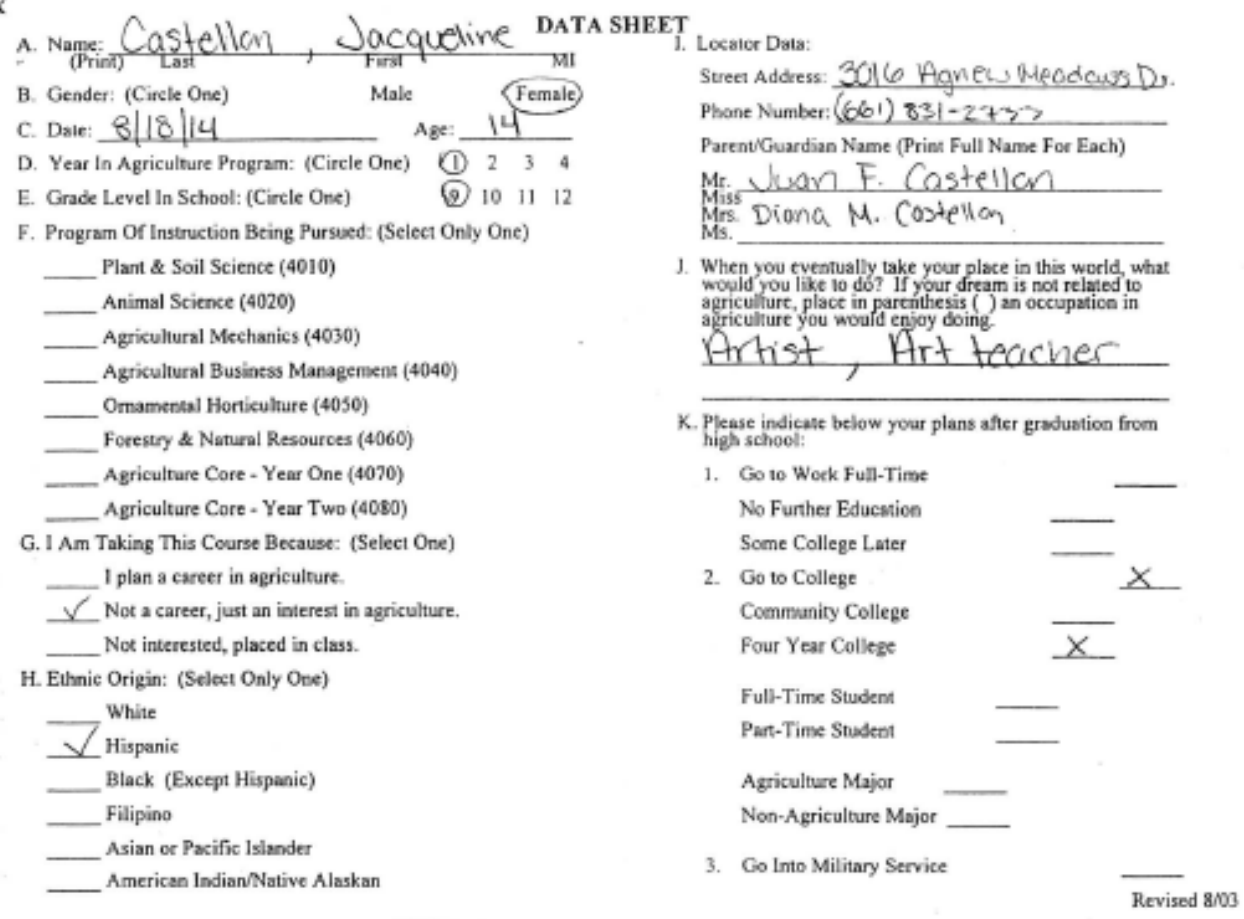

\section{STUDENT PROGRAM PLANNING FORM}

L. Planned course of study to meet occupational goal. By school year, liss all classes previously taken, currently being taken, and planned to be taken in

3.

the future.

FRESHMAN YEAR

$(20+4-15)$

\begin{tabular}{|c|c|c|c|c|c|c|c|c|c|c|c|}
\hline \multicolumn{2}{|c|}{$(2014-15)$} & \multicolumn{3}{|c|}{$(20=-1)$} & \multicolumn{2}{|c|}{$(20$} & \multicolumn{5}{|c|}{$(20--1)$} \\
\hline 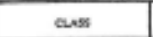 & meren & $=4$ & \begin{tabular}{l|l|l|} 
conss & \\
\end{tabular} & mecese & $m$ & coss & roose & $m$ & $\cos$ & Twowex & $m$ \\
\hline 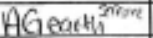 & Potterten & & Geoustly. & & & & & & & & \\
\hline PF & Borenates & & w. Histan & & & & & & & & \\
\hline Mathe & Davis & & Sponith 2 & & & & & & & & \\
\hline Somarsh & Sorrdad & & Afrindo: & & & & & & & & \\
\hline Fondish & Rawas & & Englisin & & & & & & & & \\
\hline Orchestra & Martens & & Orclustra & & & & & & & & \\
\hline & & & & & & & & & & & \\
\hline
\end{tabular}

M. Supervised Practical Experience Plan (Project program should be related to career goal).

\begin{tabular}{|c|c|c|c|c|c|c|c|}
\hline $\operatorname{sog}$ & סטw & sos & $\operatorname{sex}$ & ser & sर्र & soe & sराष \\
\hline \multicolumn{8}{|c|}{ Iandeca peruderyunt } \\
\hline & & & & & & & \\
\hline & & & & & & & \\
\hline & & & & & & & \\
\hline
\end{tabular}

N. Planned Departmental Activities (FFA)

\begin{tabular}{|l|l|l|l|}
\hline Winter Banquet & & & \\
\hline Sock fighd & & & \\
\hline & & & \\
\hline & & & \\
\hline
\end{tabular}

Parents/Guardians Signature: 
K

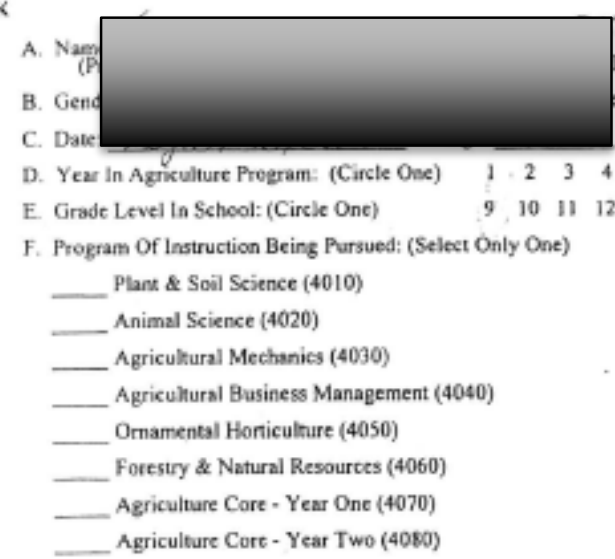

G. I Am Taking This Course Because: (Select One) I plan a career in agriculnure.

1 Not a career, just an interest in sgriculture. Not imerested, placed in class.

H. Ethnic Origin: (Select Only One)

White

Hispanic

1. Black (Excepx Hispanic)

Filapino

Asian or Pacific Islander

American lndian/Native Aleskan
A SHEET

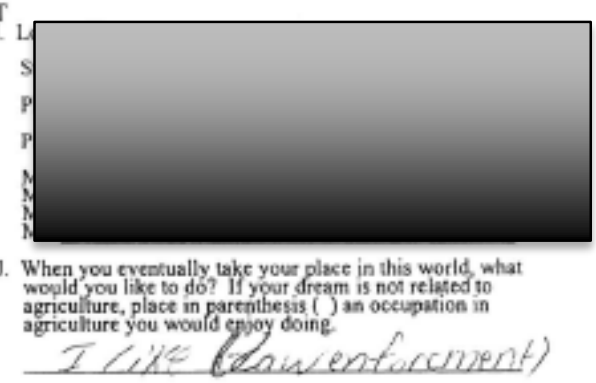

K. Please indicune below your plans after graduation from high school:

1. Go to Work Full-Time

No Furtber Education

Some College Later

2. Go to Colkze

Consmunity College

Four Year College

Full-Time Student

Part-Time Student

Agriculture Major

Non-Agriculture Msjor

3. Go Inte Military Service

Revised $8 / 03$

STUDENT PROGRAM PLANNING FORM

L. Planned ecurse of study to meet occupational gabl. By school year, list all classes previously taken, currently teing taken, and planned to be taken in

the future.

FRESHMAN YEAR

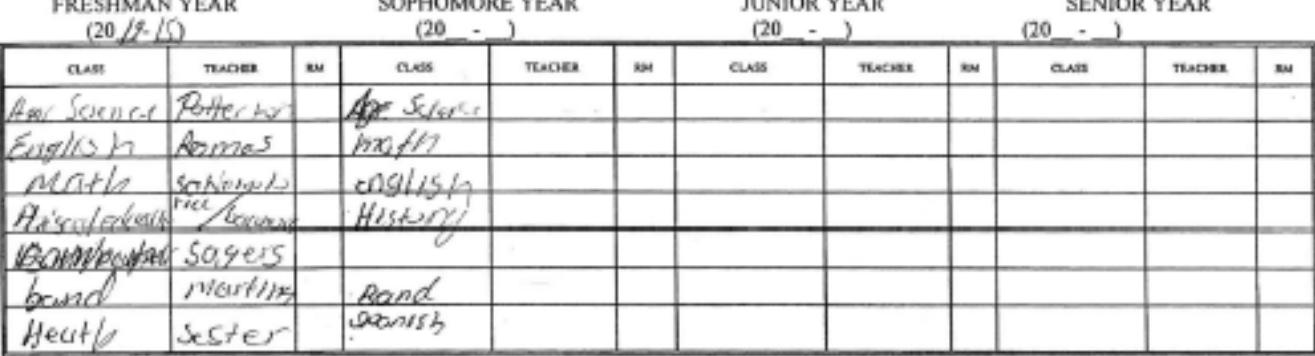

M. Supervised Practical Experience Plan (Project program should be related to career goal).

\begin{tabular}{|c|c|c|c|c|c|c|c|}
\hline 206 & $\operatorname{six}$ & $80 x$ & $\cos x$ & soe & $\operatorname{siz}$ & sot & saz \\
\hline & & & & & & & \\
\hline & & & & & & & \\
\hline & & & & & & & \\
\hline & & & & & & & \\
\hline
\end{tabular}

N. Planned Departmental Activities (FFA)

\begin{tabular}{|l|l|l|l|}
\hline & & & \\
\hline & & & \\
\hline & & & \\
\hline & & & \\
\hline & & & \\
\hline
\end{tabular}


K

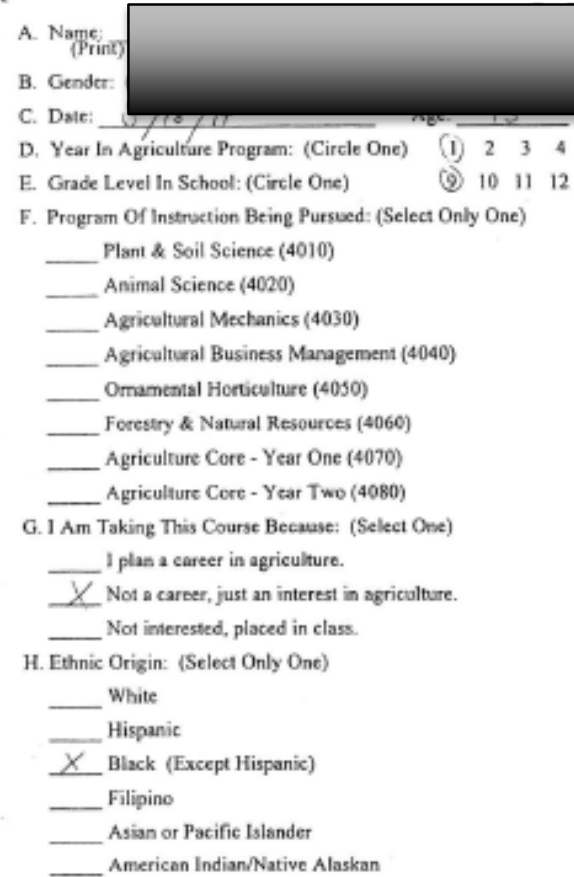

\section{EET}

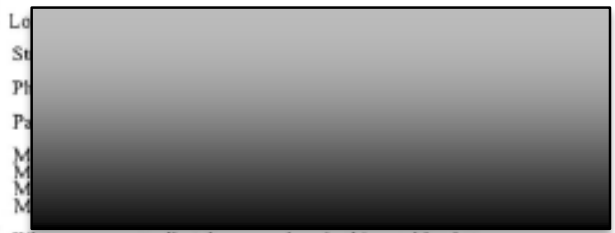

1. When you eventually take your place in this world, what
would you like in do? If your dream is nol related io agriculiture, place in parenthesis ( ) an occuption in agriculture you would enjoy doing.

I

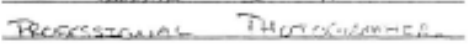
K. Please indicate below your plans after graduation from
high school:

1. Go to Work Full-Time No Furtier Education Some College Later

2. Go to College Community College Four Year College

Full-Time Student Part-Time Student

Agriculture Major

Non-Agriculture Major

3. Go Into Military Service

STUDENT PROGRAM PLANNING FORM

L. Planned course of study to meet oceupational goal. By school year, list all classes previously taken, currently being taken, and planned to be taken in

the future.

FRESHMAN YEAR

SOPHOMORE YEAR

JUNIOR YEAR

SENIOR YEAR

\begin{tabular}{|c|c|c|c|c|c|c|c|c|c|c|c|}
\hline \multicolumn{12}{|c|}{$(2014=15)$} \\
\hline and & merant & $n$ & curst & manem & $m$ & 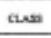 & reaser & $m$ & exs & rewoer. & $=4$ \\
\hline$\Delta G$ actet $S_{1}=$ & Remeneons & 107 & AG Breposy & & & & & & & & \\
\hline ALETESRA & Daurs & noa & Eakactsot 10 & & & & & & & & \\
\hline Euglash & MAS. $0 \alpha$ & pos & $v_{2}$ suates/anes & & & & & & & & \\
\hline RuxicALEO & TUCKER. & & 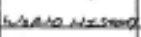 & & & & & & & & \\
\hline$S_{P A N}=5 H$ & Guso-267x & & Spanssy2 & & & & & & & & \\
\hline Compurer & Melavensa & & Geoverisy & & & & & & & & \\
\hline & & & & & & & & & & & \\
\hline
\end{tabular}

M. Supervised Practical Experience Plan (Project program should be related to career goal).

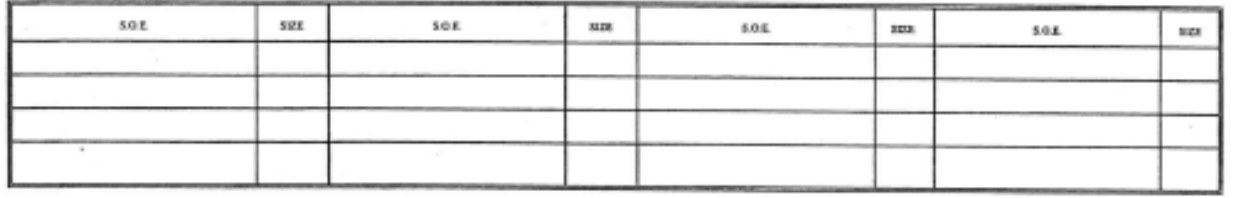

N. Planned Departmental Activities (FFA)

\begin{tabular}{|l|l|l|l|}
\hline Hozse Rescue & & & \\
\hline MeEtrauss & & & \\
\hline & & & \\
\hline & & & \\
\hline & & & \\
\hline
\end{tabular}




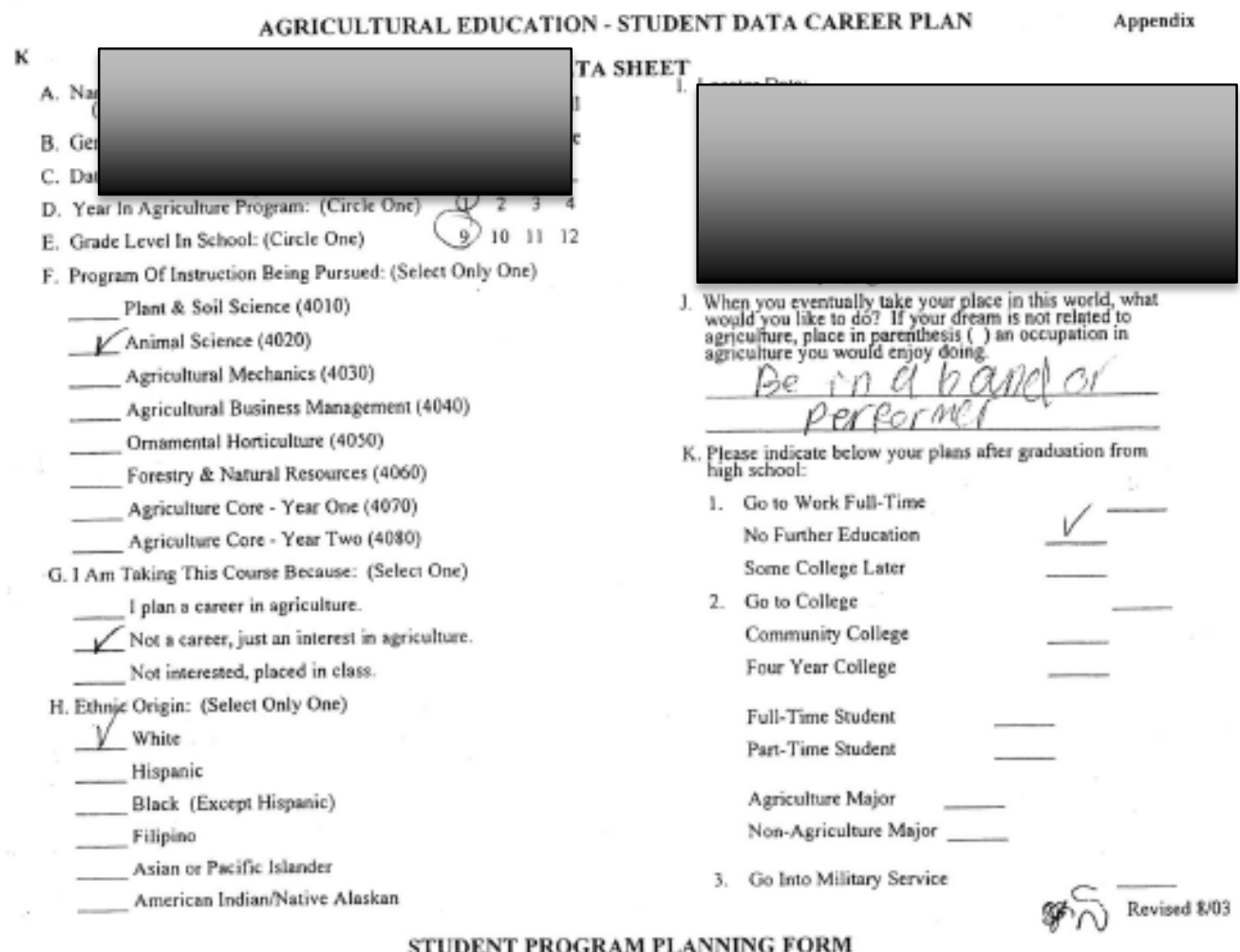

L. Planned course of siudy to meet oceupational goal. By school year, list all classes previously taken, currently being taken, and planned to be taken in

\begin{tabular}{|c|c|c|c|c|c|c|c|c|c|c|c|}
\hline \multicolumn{2}{|c|}{$\begin{array}{l}\text { the future. } \\
\text { FRESHMAN YEAR } \\
(2014-15)\end{array}$} & \multicolumn{4}{|c|}{$\begin{array}{l}\text { SOPHOMORE YEAR } \\
(20),\end{array}$} & \multicolumn{2}{|c|}{$\begin{array}{l}\text { JUNIOR YEAR } \\
(20 \quad 0)\end{array}$} & \multicolumn{4}{|c|}{ SENIOR YEAR } \\
\hline cuss & тиския & $\infty$ & auss & renese & 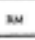 & chese & Thoes & $m$ & ans & тия & $m$ \\
\hline Ag thather & Pottertor & & A, B.meri & & & & & & & & \\
\hline Drama & Ressime & & Enomish 10 & & & & & & & & \\
\hline Englisha.6 & Kinule & & Geapetry & & & & & & & & \\
\hline P.E & Hunsaber & & Vord kaswry & & & & & & & & \\
\hline Health & Setser & & Orama & & & & & & & & \\
\hline Math & Aans & & & & & & & & & & \\
\hline CompApp & & & & & & & & & & & \\
\hline
\end{tabular}

M. Supervised Practical Experience Plan (Project program should be related to career goal).

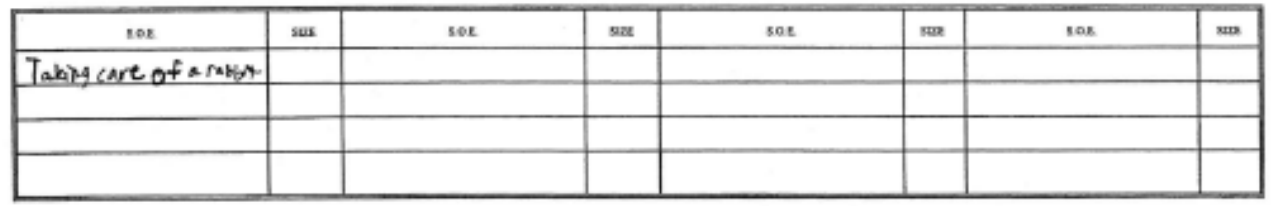

N. Planned Departmental Activities (FFA)

\begin{tabular}{|l|l|l|l|}
\hline & & & \\
\hline & & & \\
\hline & & & \\
\hline & & & \\
\hline & & & \\
\hline
\end{tabular}


Supporting Completion

Materials B: Permanent VoAg Student Records

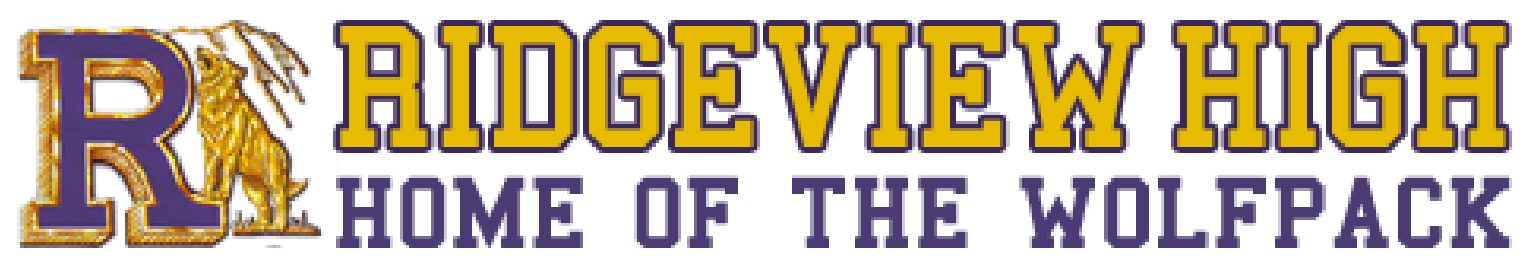




\section{B. Permanent Vo-Ag Student Records}

Permanent student files are kept in this filing cabinet in my classroom. All students in the program have a file, along with past students. Files are organized by class period and contain student data sheets, SAE records, paper record books, etc.

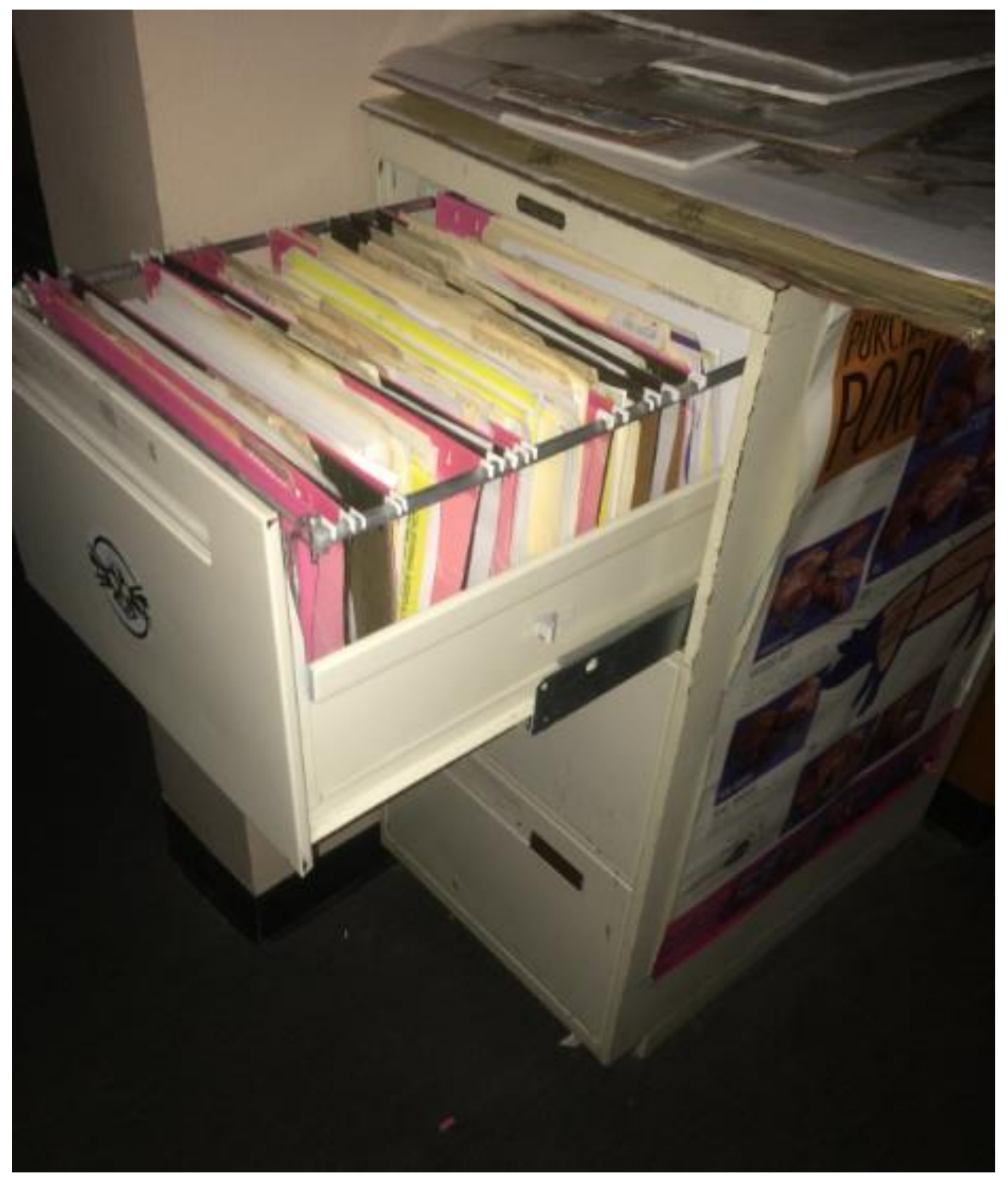




\section{Supporting Completion Materials C: Course Outlines}

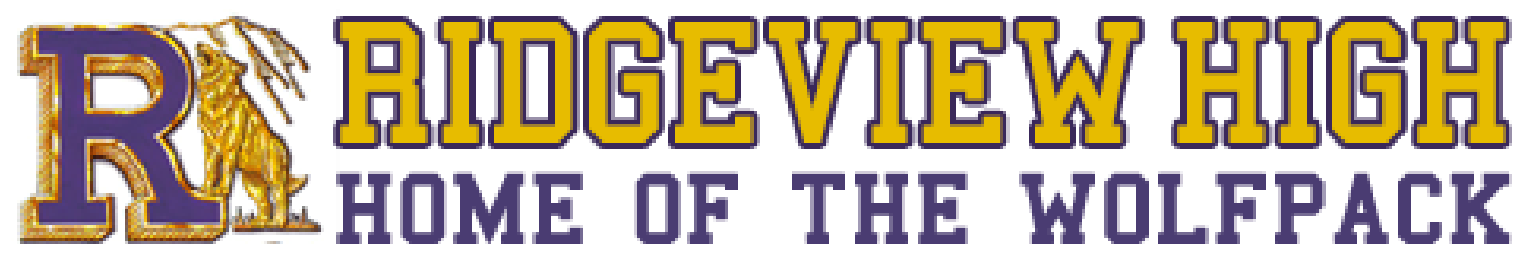




\section{Course Outlines}

Agriculture courses at my school are taken as follows:

9th grade: Ag Eath Science

$10^{\text {th }}$ grade: Ag Biology

$11^{\text {th }} \& 12^{\text {th }}$ grade (rotates every other year): Ag Sales

$11^{\text {th }} \& 12^{\text {th }}$ grade (rotates every other year): Ag Vet Science

Supporting Completion Material Attached 


\section{Agriculture Earth Resources CP}

A. COURSE INFORMATION

Grade Level:

Length of Course:

Maximum Credit:

Type:

Recommendation for Enrollment: $9^{\text {th }}$ Grade

2 semesters

10

Highly recommended for Freshman lev students

B. COURSE DESCRIPTION (Include a brief explanation of the course; mention any prerequisites, including standardized test scores; and indicate whether the course satisfies a specific gradation requirement.)

Agriculture Earth Resources is a course that meets the graduation requirement for Earth Science and is the first phase for students interested in the agriculture education program. The purpose of this course is to introduce students to the world of agriculture through the exploration of Earth Science. Students enrolled in this course will gain a deep understanding of scientific investigation and experimentation while exploring such topics as California Agriculture, Plate Tectonics, California Geology, Bio-geochemical Cycles, Earth's Atmosphere, Energy, Heat, Climate, and Astronomy. This course will also focus on leadership development, business management through the principles of accounting and computer applications, and an overview of the seven industry sectors of agricultures. Students enrolled in this course will be encouraged to participate in leadership training activities, public speaking events and become active members in the California Association Future Farmers of America (FFA). Participation in the FFA is part of their overall semester grade.

C. INSTRUCTIONAL MATERIALS (List the basic text - include title, edition, author, and copyright - and other essential supplementary materials or instructional resources/materials used in the course.) 
Miller and Levine, EARTH SCIENCE, Prentice Hall, copyright (2007)

Earth Science; Geology, the Environment, and the Universe,

Glencoe/McGraw Hill, 2002

LEADERSHIP: Personal Development and Career Success, Second Addition, Thompson Delmar publishing, 2003

DeVere Burton and Cooper, AGRISCIENCE, $4^{\text {th }}$ Edition, Thomson Delmar Publishing, 2007

\section{Supplemental Materials}

Additional materials that are not limited to, but may include:

- Computer hardware and software

- California State Agriculture Core Curriculum

- National FFA Official Manual

- FFA Student Handbooks

- California Agriculture Teacher's Association (CATA) Curricular Code

- Internet access for scientific journals and research information and interactive agriculture sites.

- Prentice Hall Earth Science Website

- Calculators

- School Farm Laboratories

- Audio / visual materials (Materials used will be those that accompany the text as well as publications by NOVA, Discovery, National Geographic, PBS, etc.)

D. COURSE OUTLINE (List the major content areas of the course an divide them according to the semester in which they fall. Designate the approximate amount of time given to each of the content areas. Suggested length: one page.)

AFS $=$ Agriculture Foundation Standards

APS = Agriculture Pathway Standards

California Adopted Earth Science Standards

\begin{tabular}{|c|l|c|l|}
\hline Unit & \multicolumn{1}{|c|}{ Topic } & Time Frame & \multicolumn{1}{c|}{$\begin{array}{c}\text { Standards } \\
\text { Addressed }\end{array}$} \\
\hline I. & California Agriculture & 2 week & APS C1.1-6 \\
\hline II. & Scientific Process & 2 weeks & $\begin{array}{l}\text { APS C13.1-3, AFS } \\
1.2 \mathrm{a}-\mathrm{m}, \text { I IE 1. a-h }\end{array}$ \\
\hline III. & $\begin{array}{l}\text { late Tectonics: ocean } \\
\text { floor features, plate } \\
\text { boundaries, rocks }\end{array}$ & 5 weeks & AFS 4.4, ES 3.a-c \\
\hline
\end{tabular}




\begin{tabular}{|c|c|c|c|}
\hline IV. & FFA & 3 week & $\begin{array}{l}\text { AFS 1.1(10.0), AFS } \\
2.1-4, \text { AFS } 7.0-6, \\
\text { AFS 9.0-6, AFS } 10.1\end{array}$ \\
\hline V. & $\begin{array}{l}\text { Plate Tectonics: } \\
\text { earthquakes, volcanoes }\end{array}$ & 2 weeks & ES 3.d, e \\
\hline VI. & Record Keeping & 2 weeks & $\begin{array}{l}\text { AFS 1.1(10.0), APS } \\
\text { C3.1-4, AFS } 10.3\end{array}$ \\
\hline VII. & California Geology & 2 week & $\begin{array}{l}\text { APS C10.1, ES 9. a- } \\
\text { c }\end{array}$ \\
\hline VIII. & Agriculture Resources & 1 week & $\begin{array}{l}\text { APS C2.1-5, ES 9. 9- } \\
\text { C }\end{array}$ \\
\hline IV. & Bio-Geochemical Cycles & 2 week & $\begin{array}{l}\text { APS C11.5-6, ES } 7 . \\
\text { a-c }\end{array}$ \\
\hline $\mathbf{X}$. & Earth's Atmosphere & 2 weeks & $\begin{array}{l}\text { APS C10.3, ES 8. a- } \\
\text { c }\end{array}$ \\
\hline $\mathbf{X I .}$ & Energy and Agriculture & 1 week & $\begin{array}{l}\text { APS E6.1\&4, ES } 4 . \\
\text { b-c }\end{array}$ \\
\hline XII. & $\begin{array}{l}\text { Heating the Earth's } \\
\text { Surface }\end{array}$ & 2 weeks & ES 5. a-e \\
\hline XIII. & Earth's Climate & 1 week & $\begin{array}{l}\text { APS E2.1-6, APS } \\
\text { F2.4, ES 6. a-c }\end{array}$ \\
\hline XIV. & Astronomy & 2weeks & ES 1.a-f \& 2.a-d \\
\hline $\mathbf{X V}$. & $\begin{array}{l}\text { Soils: formation, texture, } \\
\text { structure, erosion, and } \\
\text { conservation of }\end{array}$ & 4 weeks & APS E3.2\&4 \\
\hline XVII & $\begin{array}{l}\text { Supervised Agriculture } \\
\text { Experience }\end{array}$ & 2 weeks & $\begin{array}{l}\text { APS C4.1-5, C6.1-2, } \\
\text { C8.1-3, C9.1-5, } \\
\text { C11.1, AFS 10.2 }\end{array}$ \\
\hline XVIII & $\begin{array}{l}\text { Agricultural Careers and } \\
\text { Development Events }\end{array}$ & 2 weeks & $\begin{array}{l}\text { AFS 3.1-6, AFS 5.0- } \\
3, \text { AFS } 11.0\end{array}$ \\
\hline
\end{tabular}

E. COURSE OBJECTIVES FOR (The objectives area to include the specific, major skills or understandings which students will be able to demonstrate or acquire instruction in the course. A minimum of eight to twelve objectives should be identified for each semester of the course. Each objective is to be clearly linked to the Board adopted standards for the course or subject area; indicate the link by placing the number of the appropriate standards (s) after each objective. Minimum length: one page)

- Specific applications of Algebra I standards (grades eight through twelve): AFS 1.1

B. $\quad 10.0 \quad$ Students add, subtract, multiply, and divide monomials and polynomials. Students solve multistep problems, including word problems, by using these techniques.

- Specific applications of Investigation and Experimentation standards (grades nine through twelve): AFS 1.2 
1.a Select and use appropriate tools and technology (such as computer-linked probes, spreadsheets, and graphing calculators) to perform tests, collect data, analyze relationships, and display data.

1.c Identify possible reasons for inconsistent results, such as sources of error or uncontrolled conditions.

1.d Formulate explanations by using logic and evidence.

1.f Distinguish between hypothesis and theory as scientific terms.

1.j Recognize the issues of statistical variability and the need for controlled tests.

1.I Analyze situations and solve problems that require combining and applying concepts from more than one area of science.

1.m Investigate a science-based societal issue by researching the literature, analyzing data, and communicating the findings. Examples of issues include irradiation of food, cloning of animals by somatic cell nuclear transfer, choice of energy sources, and land and water use decisions in California.

- Students understand the principles of effective oral, written, and multimedia communication in a variety of formats and contexts. AFS 2.0-2.4

- Students understand how to make effective decisions, use career information, and manage personal career plans: AFS 3.0

3.1 Know the personal qualifications, interests, aptitudes, information, and skills necessary to succeed in careers.

3.2 Understand the scope of career opportunities and know the requirements for education, training, and licensure.

3.3 Develop a career plan that is designed to reflect career interests, pathways, and postsecondary options.

3.4 Understand the role and function of professional organizations, industry associations, and organized labor in a productive society.

3.5 Understand the past, present, and future trends that affect careers, such as technological developments and societal trends, and the resulting need for lifelong learning.

3.6 Know important strategies for self-promotion in the hiring process, such as job applications, résumé writing, interviewing skills, and preparation of a portfolio.

- Students understand how to create alternative solutions by using critical and creative thinking skills, such as logical reasoning, analytical thinking, and problem-solving techniques: AFS 5.0 
5.1 Apply appropriate problem-solving strategies and critical thinking skills to work-related issues and tasks.

5.2 Understand the systematic problem-solving models that incorporate input, process, outcome, and feedback components.

5.3 Use critical thinking skills to make informed decisions and solve problems.

- Students know the behaviors associated with the demonstration of responsibility and flexibility in personal, workplace, and community settings: AFS 7.0

7.1 Understand the qualities and behaviors that constitute a positive and professional work demeanor.

7.2 Understand the importance of accountability and responsibility in fulfilling personal, community, and workplace roles.

7.3 Understand the need to adapt to varied roles and responsibilities.

7.4 Understand that individual actions can affect the larger community.

7.5 Understand the importance of time management to fulfill responsibilities.

7.6 Know how to apply high-quality craftsmanship to a product or presentation and continually refine and perfect it.

- Students understand effective leadership styles, key concepts of group dynamics, team and individual decision making, the benefits of workforce diversity, and conflict resolution: AFS 9.0

9.1 Understand the characteristics and benefits of teamwork, leadership, and citizenship in the school, community, and workplace settings.

9.2 Understand the ways in which pre-professional associations, such as the Future Farmers of America (FFA), and competitive career development activities enhance academic skills, promote career choices, and contribute to employability.

9.3 Understand how to organize and structure work individually and in teams for effective performance and the attainment of goals.

9.4 Know multiple approaches to conflict resolution and their appropriateness for a variety of situations in the workplace.

9.5 Understand how to interact with others in ways that demonstrate respect for individual and cultural differences and for the attitudes and feelings of others. 
9.6 Understand leadership, cooperation, collaboration, and effective decision-making skills applied in group or team activities, including the student organization.

- Students understand the essential knowledge and skills common to all pathways in the Agriculture and Natural Resources sector: AFS 10.0

10.1 Understand the aims, purposes, history, and structure of the FFA student organization, and know the opportunities it makes available.

10.2 Manage and actively engage in a career-related, supervised agricultural experience.

10.3 Understand the importance of maintaining and completing the California Agricultural Record Book.

10.4 Maintain and troubleshoot equipment used in the agricultural industry.

- Students understand the role of agriculture in the California economy: APS C1.0

C1.1 Understand the history of the agricultural industry in California.

C1.2 Understand how California agriculture affects the quality of life.

C1.3 Understand the interrelationship of California agriculture and society at the local, state, national, and international levels.

C1.4 Understand the economic impact of leading California agricultural commodities.

C1.5 Understand the economic impact of major natural resources in California.

C1.6 Know the economic importance of major agricultural exports and imports.

- Students understand the inter-relationship between agriculture and the environment: APS C2.0

C2.1 Understand important agricultural environmental impacts on soil, water, and air.

C2.2 Understand current agricultural environmental challenges.

C2.3 Understand how natural resources are used in agriculture.

C2.4 Compare and contrast practices for conserving renewable and nonrenewable resources.

C2.5 Understand how new energy sources are developed from agricultural products (e.g., gas-cogeneration and ethanol).

- Students understand the importance of animals, the domestication of animals, and the role of animals in modern society: APS C4.0 
C4.1 Understand the evolution and roles of domesticated animals in society.

C4.2 Know the differences between domestication and natural selection.

C4.3 Understand the modern-day uses of animals and animal by-products.

C4.4 Understand various points of view regarding the use of animals.

C4.5 Understand unique and alternative uses of animals (e.g., Handi-Riders and companion animals).

- Students understand animal anatomy and systems: APS C6.0

C6.1 Know the names and locations of the external anatomy of animals.

C6.2 Know the anatomy and major functions of vertebrate systems, including digestive, reproductive, circulatory, nervous, muscular, skeletal, respiratory, and endocrine systems.

- Students understand fundamental animal nutrition and feeding: APS C8.0

C8.1 Know types of nutrients required by farm animals (e.g., proteins, minerals, vitamins, carbohydrates, fats/oils, water).

C8.2 Analyze suitable common feed ingredients, including forages, roughages, concentrates, and supplements, for ruminant, monogastric, equine, and avian digestive systems.

C8.3 Understand basic animal feeding guidelines and evaluate sample feeding programs for various species, including space requirements and economic considerations.

- Students understand basic animal health: APS C9.0

C9.1 Assess the appearance and behavior of a normal, healthy animal.

C9.2 Understand the ways in which housing, sanitation, and nutrition influence animal health and behavior.

C9.3 Understand the causes and control of common animal diseases.

C9.4 Understand how to control parasites and why.

C9.5 Understand the legal requirements for the procurement, storage, methods of application, and withdrawal times of animal medications and know proper equipment handling and disposal techniques.

- Students understand soil science principles: APS C10.0

C10.1 Recognize the major soil components and types. 
C10.3 Understand water delivery and irrigation system options.

- Students understand plant growth and development: APS C11.0

C11.1 Understand the anatomy and functions of plant systems and structures.

C11.5 Understand the photosynthesis process and the roles of the sun, chlorophyll, sugar, oxygen, carbon dioxide, and water in the process.

C11.6 Understand the respiration process in the breakdown of food and organic matter.

- Students understand the scientific method: APS C13.0

C13.1 Understand the steps of the scientific method.

C13.2 Analyze an animal or plant problem and devise a solution based on the scientific method.

C13.3 Use the scientific method to conduct agricultural experiments.

- Students understand air and water use, management practices, and conservation strategies: APS E2.0

E2.1 Understand the government's role in regulating air, soil, and water use management practices and conservation strategies.

E2.2 Understand air and water conservation issues.

E2.3 Understand appropriate water conservation measures.

E2.4 Understand the component of a plan that monitors water quality.

E2.5 Understand the component of a plan that monitors air quality.

E2.6 Analyze the way in which water management affects the environment and human needs.

- Students understand soil composition and soil management: APS E3.0

E3.2 Understand the reasons for and importance of soil conservation.

E3.4 Understand how to develop and implement a soil management plan for a natural resource management area.

- Students understand aquatic resource use and management: APS E6.0

E6.1 Understand the different types of aquatic resources.

E6.4 Analyze the relationship between water quality and aquatic species habitat.

- Students understand plant physiology and growth principles: APS F2.0 
F2.4 Understand the factors that influence plant growth, including water, nutrients, light, soil, air, and climate.

- The evidence from Earth and moon rocks indicates that the solar system was formed from a nebular cloud of dust and gas approximately 4.6 billion years ago. ES 1.b

- The evidence from geological studies of Earth and other planets suggest that the early Earth was very different from Earth today. ES 1.c

- The evidence indicating that the planets are much closer to Earth than the stars are. ES 1.d

- The Sun is a typical star and is powered by nuclear reactions, primarily the fusion of hydrogen to form helium. ES 1.e

- The evidence for the dramatic effects that asteroid impacts have had in shaping the surface of planets and their moons and in mass extinctions of life on Earth. ES 1.f

- The solar system is located in an outer edge of the disc-shaped Milky Way galaxy, which spans 100,000 light years. ES 2.a

- Galaxies are made of billions of stars and comprise most of the visible mass of the universe. ES 2.b

- The evidence indicating that all elements with an atomic number greater than that of lithium have been formed by nuclear fusion in stars. ES 2.C

- That stars differ in their life cycles and that visual, radio, and X-ray telescopes may be used to collect data that reveal those differences. ES 2.d

- Features of the ocean floor (magnetic patterns, age, and sea-floor topography) provide evidence of plate tectonics. ES 3.a

- The principal structures that form at the three different kinds of plate boundaries. ES 3.b

- How to explain the properties of rocks based on the physical and chemical conditions in which they formed, including plate tectonic processes. ES 3.c

- Why and how earthquakes occur and the scales used to measure their intensity and magnitude. ES 3.d

- There are two kinds of volcanoes: one kind with violent eruptions producing steep slopes and the other kind with voluminous lava flows producing gentle slopes. ES 3.e

- The relative amount of incoming solar energy compared with Earth's internal energy and the energy used by society. ES 4.a

- The fate of incoming solar radiation in terms of reflection, absorption, and photosynthesis. ES 4.b 
- The different atmospheric gases that absorb the Earth's thermal radiation and the mechanism and significance of the greenhouse effect. ES 4.c

- How differential heating of Earth results in circulation patterns in the atmosphere and oceans that globally distribute the heat. ES 5.a

- The relationship between the rotation of Earth and the circular motions of ocean currents and air in pressure centers. ES 5.b

- The origin and effects of temperature inversions. ES 5.C

- Properties of ocean water, such as temperature and salinity, can be used to explain the layered structure of the oceans, the generation of horizontal and vertical ocean currents, and the geographic distribution of marine organisms. ES 5.d

- Rain forests and deserts on Earth are distributed in bands at specific latitudes. ES 5.e

- Weather (in the short run) and climate (in the long run) involve the transfer of energy into and out of the atmosphere. ES 6.a

- The effects on climate of latitude, elevation, topography, and proximity to large bodies of water and cold or warm ocean currents. ES 6.b

- How Earth's climate has changed over time, corresponding to changes in Earth's geography, atmospheric composition, and other factors, such as solar radiation and plate movement. ES 6.c

- The carbon cycle of photosynthesis and respiration and the nitrogen cycle. ES 7.a

- The global carbon cycle: the different physical and chemical forms of carbon in the atmosphere, oceans, biomass, fossil fuels, and the movement of carbon among these reservoirs. ES 7.b

- The movement of matter among reservoirs is driven by Earth's internal and external sources of energy. ES 7.c

- The thermal structure and chemical composition of the atmosphere. ES 8.a

- How the composition of Earth's atmosphere has evolved over geologic time and know the effect of outgassing, the variations of carbon dioxide concentration, and the origin of atmospheric oxygen. ES 8.b

- The location of the ozone layer in the upper atmosphere, its role in absorbing ultraviolet radiation, and the way in which this layer varies both naturally and in response to human activities. ES 8.C

- The resources of major economic importance in California and their relation to California's geology. ES 9.a

- The principal natural hazards in different California regions and the geologic basis of those hazards. ES 9.b 
- The importance of water to society, the origins of California 's fresh water, and the relationship between supply and need. ES 9.c

- Select and use appropriate tools and technology (such as microscopes, computer-linked probes, computer software, and scientific calculators) in a safe manner.I \& $E$ 1a

- Select and use appropriate tools and technology (such as microscopes, computer-linked probes, computer software, and scientific calculators) in a safe manner.I \& $\mathrm{E} 1 \mathrm{a}$

- Develop hypotheses, perform tests, collect data, display data, analyze relationships, and draw conclusions in order to solve problems. I \& $\mathrm{E}$ 1a, d, l, j

- Analyze situations and solve problems that require combining concepts from more than one area of science. I \& E 1 I

- How the differences and similarities among the sun, the terrestrial planets, and the gas planets may have been established during the formation of the solar system. ES 1.a

F. STUDENT EVALUATION STANDARDS (List the criteria on which students will be graded in the course. Give the approximate weight for each of the grading criteria in determining the student's grade, such as tests, homework, labs, class participation. Also indicate the weight given to quarter grades and semester final in tabulating the final grade.)

C.

Assignments and labs $\begin{array}{ll}\text { Assessments } & 30 \% \\ \text { FFA Participation } & 10 \%\end{array}$

$60 \%$

G. SUGGESTED INSTRUCTIONAL ACTIVITIES (This item is optional and is not required of the course of study. If it is completed, it should include teacher and/or student activities such as field trips, demonstrations, speakers, or special procedures that will assist the students in learning the course objectives.)

Prepared by Craig Davidson, Ralph Mendes, Travis Wyrick, and Ric Lemucchi 
District Wide Course of Study Title:

\section{Agriculture (AG) Biology}

\section{H. COURSE INFORMATION}

Grade Level: 9-10

Length of Course: One Year (two semesters)

Maximum Credit: 10 hours

Type: CP/GATE

Recommendation for Enrollment: Freshman level science course or equivalent.

\section{B. COURSE DESCRIPTION}

Biology is a one-year laboratory-based course designed to provide students with a basic understanding of the characteristics and functions of living organisms as well as provide students with the opportunity to develop their skills in scientific investigation, which will include projects requiring research. This course satisfies one half of the science requirements for graduation as well as the UC "AG" requirement for laboratory science. It is designed to give advanced students a greater in-depth look into the characteristics and functions of living organisms. Topics include scientific methodology, cellular structure and function, evolutionary processes, genetics, ecology, anatomy and physiology. The Agriscience Pathway helps students acquire a broad understanding of a variety of agricultural areas, develop an awareness of the many career opportunities in agriculture, participate in occupationally relevant experiences, and work cooperatively with a group to develop and expand leadership abilities. Students study California agriculture, agricultural business, agricultural technologies, natural resources, and animal, plant, and soil sciences.

\section{E. C. BOARD-ADOPTED TEXTBOOKS}


Miller and Levine, Biology, Prentice Hall, copyright (2007)

McLaren, Rotundo, and Gurley, Heath Biology (1991), Danville, Illinois:

Interstate Publishers.

\section{F. D. SUPPLEMENTARY INSTRUCTIONAL MATERIALS}

Osborne, Edward, Agriscience: Fundamentals and Applications (1990), Albany, New York: Delmar Publishers. Osborne, Edward, Biological Science Applications in Agriculture (1994) Danville, Illinois: Interstate Publishers. Prentice Hall Lab Manual, study guides, charts, models, supplemental reading materials (including magazines and journals), multiple audio-visual materials, computer hardware and software, Internet access, demonstration materials, living and preserved specimens, various laboratory equipment, and PowerPoint Presentations.

\section{G. E. COURSE OUTLINE}

\begin{tabular}{|c|c|c|c|}
\hline Unit/Quarter & Topic & $\begin{array}{c}\text { California } \\
\text { State Standards }\end{array}$ & $\begin{array}{l}\text { CTE/Agriculture } \\
\text { Standards }\end{array}$ \\
\hline Quarter 1 & $\begin{array}{l}\text { Scientific } \\
\text { Process }\end{array}$ & $\begin{array}{l}\text { I\&E c,d,f,j,j,; Bio } \\
4 \mathrm{e}, 5 \mathrm{a}, 9 \mathrm{a}\end{array}$ & $\begin{array}{l}\text { 1.a,c, d,f,j,l,m } \\
\mathrm{C} 13.0 ; \mathrm{C} 13.1 ; \mathrm{C} 13.2 ; \mathrm{C} 13.3\end{array}$ \\
\hline Quarter 3 & Ecology & Bio 6a-f & $\begin{array}{l}\text { C2.1; C2.3; G1.0; G1.4; } \\
\text { D7.0; D7.2; E1.0 }\end{array}$ \\
\hline III. Quarter 1 & $\begin{array}{l}\text { Cellular Structure } \\
\text { and Process }\end{array}$ & Bio $1 c, e, f, g, h, l, j, 2 a$ & $\begin{array}{l}\text { C5.0; C5.1; C5.2; C5.3; C5.4 } \\
\text { C11.5; C11.4; C11.0 }\end{array}$ \\
\hline IV. Quarter 2 & Genetics & $\begin{array}{l}\text { Bio 1d, 2a-g, 3a-b, 4a- } \\
\text { e, 5a-c, 7b }\end{array}$ & $\begin{array}{l}\text { C7.0; D5.0; D5.4; D5.5; } \\
\text { C3.3; C3.4 }\end{array}$ \\
\hline V. $\quad$ Quarter 3 & Evolution & Bio 7a-d, 8a-e & C4.1; C4.2 \\
\hline X. Quarter 3-4 & $\begin{array}{l}\text { Human Anatomy } \\
\text { and Physiology }\end{array}$ & Bio 9b-e, 10a-e & C6.0; C11.5; \\
\hline Quarter 4 & $\begin{array}{l}\text { Independent Lab } \\
\text { Based Projects, } \\
\text { CTE }\end{array}$ & $\begin{array}{l}\text { Bio 9a-l, } \\
\text { I\&Ea,b,c,d,f,g,k,l,m }\end{array}$ & $\begin{array}{l}\text { 2.0 - Communication:2.1, } \\
2.2,2.3,2.7,2.8, \text { Writing: } \\
1.3,1.5,2.3,2.5,2.6, \\
\text { Writing Strategies \& } \\
\text { Applications: } 1.6,2.6 \\
\text { Listening \& Speaking Skills: } \\
1.1,1.7 \\
\text { 9.0 - Leadership \& Team } \\
\text { Work: } 9.1,9.2,9.3, \\
10.0 \text { - Technical Knowledge } \\
\text { \& Skills: } 10.2,10.3,\end{array}$ \\
\hline
\end{tabular}

\section{H. F. COURSE OBJECTIVES FOR Biology}

As part of the California State Standards for Biology/Life Science and Investigation and Experimentation, students will: 
- Select and use appropriate tools and technology (microscopes, computerlinked probes, computer software, and scientific calculators) in a safe manner. I\&E 1a.

- Develop hypotheses, perform tests, collect data, display data, analyze relationships, and draw conclusions in order to solve problems. I\&E 1a,d,l,j.

- Analyze situations and solve problems that require combining concepts from more than one area of science. I\&E 11 .

- Use the Periodic Table of the Elements to develop models of the atoms important to living organisms. Chem1a,d, Cell Bio KHSD.

- Know that the bonding characteristics of carbon lead to a large variety of structures ranging from simple hydrocarbons to complex biological molecules such as carbohydrates, proteins, enzymes, lipids, and nucleic acids. Bio 1h, Chem 2b.

- Know that the cell is the structural unit of life. Bio 1a-h, 5a-b.

- Know that fundamental life processes of plants and animals depend on a variety of chemical reactions that are carried out by various organelles in cells. Bio 1b,f,g,i.

- Know that cells are enclosed within semi-permeable membranes that regulate their interaction with their surroundings. Bio 1a.

- Know how energy is obtained and utilized by the cell and how the processes of photosynthesis and respiration are important to living organisms. Bio 1a.

- Know how prokaryote and eukaryote cells and viruses differ in complexity, and how plant and animal cells and bacteria differ in their general structure. Bio 1f,g.

- Know that there are important differences between bacteria and viruses, with respect to their requirements for growth and replication, the primary defense of host organisms against them, and the effective treatment of infections they cause. Bio 1c, 10a-e.

- Understand that genes are a set of instructions, encoded in the DNA sequence of each organism, which specify the sequence of amino acids in proteins characteristic of that organism. Bio 1d, 4a-e, 5a-b.

- Know hoe cells grow and reproduce through the processes of mitosis and meiosis. Bio 2a-b.

- Understand basic DNA technology, such as recombination DNA procedures, forensic science, and gel electrophoresis. Bio 1d, 4a-f.

- Analyze both the positive and negative impacts of genetic engineering on society. Bio 2a-b, 5c. 
- Know the basic principles of Mendelian genetics used to predict the phenotype and genotype of offspring in genetic crosses. Bio 2a-g, a-b.

- Know hoe living organisms interrelate with one another and their non-living environment. Bio 6a-f.

- Know how water, carbon, and nitrogen cycle between abiotic resources and organic matter in the ecosystem and how oxygen cycles via photosynthesis and respiration. Bio 1f, 6d-f.

- Know how to analyze changes in an ecosystem as a result of ecological disrupters such as natural disasters, human activity, and introduction of nonnative species. Bio 6b-c.

- Know how changing environments and evolutionary processes result in genetic change. Bio 6b, 7a-d, 8a-e.

- Know how to determine if genetic change had occurred in a population using the Hardy-Weinberg equation. Bio 7e-f.

- Know the interrelationships among tissues, organs, and systems of plants and animals. Bio 9a-e, 10a-e.

- Know the structure and function of the major systems, their role in homeostasis, and comparisons between organisms. Bio 9a-e.

- Understand the various mechanisms organisms have for combating disease by developing a knowledge of the human immune response. Bio 10a-e.

- Know the biodiversity is the sum total of different kinds of organisms and is affected by alterations of habitats. Bio 6 a.

\section{CTE Course Objectives for AG Biology}

\section{Foundation Standards}

2.0 Communications: Students understand the principles of effective oral, written, and multimedia communication in a variety of formats and contexts.

2.1 Analyze the structure and format of functional workplace documents, including the graphics and headers, and explain how authors use the features to achieve their purposes.

2.2 Prepare a bibliography of reference materials for a report using a variety of consumer, workplace, and public documents.

2.3 Generate relevant questions about readings on issues that can be researched

2.6 Demonstrate use of sophisticated learning tools by following technical directions (e.g., those found with graphic calculators and specialized software programs and in access guides to World Wide Web sites on the Internet). 
2.7 Critique the logic of functional documents by examining the sequence of information and procedures in anticipation of possible reader misunderstandings.

2.8 Evaluate the credibility of an author's argument or defense of a claim by critiquing the relationship between generalizations and evidence, the comprehensiveness of evidence, and the way in which the author's intent affects the structure and tone of the text (e.g., in professional journals, editorials, political

\subsection{Writing:} speeches, primary source material).

1.3 Use clear research questions and suitable research methods (e.g., library, electronic media, personal

interview) to elicit and present evidence from primary and secondary sources.

1.5 Synthesize information from multiple sources and identify complexities and discrepancies in the

information and the different perspectives found in each medium (e.g., almanacs, microfiche, news

sources, in-depth field studies, speeches, journals, technical documents).

2.3 Write expository compositions, including analytical essays and research reports:

a. Marshal evidence in support of a thesis and related claims, including information on all relevant perspectives.

b. Convey information and ideas from primary and secondary sources accurately and coherently.

c. Make distinctions between the relative value and significance of specific data, facts, and ideas.

d. Include visual aids by employing appropriate technology to organize and record information on charts, maps, and graphs.

e. Anticipate and address readers' potential misunderstandings, biases, and expectations.

f. Use technical terms and notations accurately.

2.5 Write business letters:

a. Provide clear and purposeful information and address the intended audience appropriately.

b. Use appropriate vocabulary, tone, and style to take into account the nature of the relationship with, and the knowledge and interests of, the recipients.

c. Highlight central ideas or images.

2.6 Write technical documents (e.g., a manual on rules of behavior for conflict resolution, procedures for

conducting meeting, minutes of a meeting): Report information and convey ideas logically and correctly.

Offer detailed and accurate specifications. Include scenarios, definitions, and examples to aid

comprehension (e.g., troubleshooting guide). Anticipate readers' problems, mistakes, and 
misunderstandings.

\subsection{Listening and Speaking:}

1.1. Formulate judgments about the ideas under discussion and support those judgments with convincing evidence.

1.7 Use props, visual aids, graphs, and electronic media to enhance the appeal and accuracy of presentations.

9.0 Leadership and Teamwork: Students understand effective leadership styles, key concepts of group dynamics, team

and individual decision making, the benefits of workforce diversity, and conflict resolution:

9.1 Understand the characteristics and benefits of teamwork, leadership, and citizenship in the school, community, and workplace settings.

9.2 Understand the ways in which pre-professional associations, such as the Future Farmers of America (FFA), and competitive career development activities enhance academic skills, promote career choices, and contribute to employability.

9.3 Understand how to organize and structure work individually and in teams for effective performance and the attainment of goals.

10.0 Technical Knowledge and Skills: Students understand the essential knowledge and skills common to all pathways in

the Agriculture and Natural Resources sector:

10.1 Understand the aims, purposes, history, and structure of the FFA student organization, and know the opportunities it makes available.

10.2 Manage and actively engage in a career-related, supervised agricultural experience. 10.3 Understand the importance of maintaining and completing the California Agricultural Record Book.

\section{Agriculture Standards}

The Agriscience Pathway helps students acquire a broad understanding of a variety of agricultural areas, develop an awareness of the many career opportunities in agriculture, participate in occupationally relevant experiences, and work cooperatively with a group to develop and expand leadership abilities. Students study California agriculture, agricultural business, agricultural technologies, natural resources, and animal, plant, and soil sciences. 
C2.0 Students understand the interrelationship between agriculture and the environment:

C2.1 Understand important agricultural environmental impacts on soil, water, and air.

C2.3 Understand how natural resources are used in agriculture.

\section{C3.0 Students understand the effects of technology on agriculture:}

C3.3 Understand public concern for technological advancements in agriculture, such as genetically

modified organisms.

C3.4 Understand the laws and regulations concerning biotechnology.

C4.0 Students understand the importance of animals, the domestication of animals, and the role of

animals in modern society:

C4.1 Understand the evolution and roles of domesticated animals in society.

C4.2 Know the differences between domestication and natural selection.

C5.0 Students understand the cell structure and function of plants and animals:

C5.1 Understand the purpose and anatomy of cells.

C5.2 Know how cell parts function.

C5.3 Understand various cell actions, such as osmosis and cell division.

C5.4 Understand how plant and animal cells are alike and different.

\section{C6.0 Students understand animal anatomy and systems:}

C6.1 Know the names and locations of the external anatomy of animals.

C6.2 Know the anatomy and major functions of vertebrate systems, including digestive, reproductive,

circulatory, nervous, muscular, skeletal, respiratory, and endocrine systems

\section{C7.0 Students understand basic animal genetics:}

C7.1 Differentiate between genotype and phenotype, and describe how dominant and recessive genes function.

C7.2 Compare genetic characteristics among cattle, sheep, swine, and horse breeds.

C7.3 Understand how to display phenotype and genotype ratios (e.g., by using a Punnett Square).

C7.4 Understand the fertilization process.

C7.5 Understand the purpose and processes of mitosis and meiosis.

\section{C11.0 Students understand plant growth and development}

C11.4 Examine plant sexual and asexual reproduction.

C11.5 Understand the photosynthesis process and the roles of the sun, chlorophyll, sugar, oxygen, carbon dioxide, and water in the process.

C11.6 Understand the respiration process in the breakdown of food and organic matter.

C13.0 Students understand the scientific method:

C13.1 Understand the steps of the scientific method. 
C13.2 Analyze an animal or plant problem and devise a solution based on the scientific method.

C13.3 Use the scientific method to conduct agricultural experiments.

D5.0 Students understand animal inheritance and selection principles, including the structure and role of DNA:

D5.4 Understand how to predict phenotypic and genotypic results of a dominant and recessive gene pair.

D5.5 Understand the role of mutations (both naturally occurring and artificially induced) and hybrids in animal

genetics.

D7.0 Students understand common rangeland management practices and their impact on a balanced ecosystem.

D7.2 Know how rangeland management practices affect pasture production, erosion control, and the general

balance of the ecosystem.

E1.0 Students understand the importance of energy and energy cycles:

E1.1 Understand the oxygen, carbon, nitrogen, and water cycles.

G1.0 Students understand plant classification principles:

G1.4 Understand the differences between and uses of native and nonnative plants.

G2.0 Students understand cell biology:

G2.1 Understand the differences between prokaryotic cells and plant and animal eukaryotic cells and how viruses differ from them in complexity and general structure.

\section{J. G. STUDENT EVALUATION STANDARDS}

a. Common unit pre/post testing

b. KHSD benchmark assessments

c. Teacher's tests and quizzes

d. Standardized tests (state, federal)

e. Class assignments, activities, and research projects

f. Check lists

g. Homework/classwork

h. Laboratory assessment and analysis

i. Audio/visual media presentations

\section{Assessment Criteria}

Grading Scale

$A=90-100 \%$

$B=80-90 \%$

C $=70-79 \%$ 


$$
\begin{aligned}
& D=60-69 \% \\
& F=0-59 \%
\end{aligned}
$$

\section{Quarter Grade Determination}

All class activities and assignments $\quad 30 \%$

CTE (FFA, SAE)

Labs and research projects

Quizzes/Tests
$10 \%$

$30 \%$

$30 \%$

\section{Semester Grade Determination}

Quarter 1 Grade

$40 \%$

Quarter 2 Grade

$40 \%$

Semester Exam

$20 \%$

\begin{tabular}{ll} 
& $\begin{array}{l}\text { LeAnn Riley } \\
\text { Jason Ferreira } \\
\text { Jessica Helm }\end{array}$ \\
\hline
\end{tabular}

Date: $\underline{02 / 01 / 2012}$ 
DPC\#:

Approved by Board:

\section{COURSE OF STUDY}

Agriculture 3 - Veterinary Science

Course Title

(Title must corr
What A-G Requirement met?

Does this course satisfy a graduation requirement in another subject area?

\section{$11 / 12$ Grade Level} de Catalog)
10 uni Max Cre
Prepared by: $\quad$ Amie Mertz School:
U.C. Approved ? College Prep, U.C. Approved

If so, what subject area?

\section{D}

No

Approval of Site Administrator:

Date:

I. COURSE INFORMATION

Grade Level:

Length of Course:

Maximum Credit:

Type:

Recommendation for Enrollment:
$11 / 12$

1 year

Ten Units

Miscellaneous

Enrollment in Agriculture Program with prior completic of Agriculture Biology with Algebra 1 strong recommended.

\section{K. BRIEF DESCRIPTION OF THE COURSE}

The Veterinary Science course is designed to provide students with an opportunity to investigate different aspects of the animal health and care occupations, or to continue on in post-secondary education in the animal science field. This content of this course will include: job-search skills, comparative anatomy and physiology, animal reproduction, animal inheritance and selection principles, basic pet grooming skills, animal restraint, nutrition and housing, medical terminology, animal welfare 
concerns, production practices for large and small animals, production of small animals, how animal products and by-products are processed and marketed, species and breed identification, and disease control/management. This course will also combine fundamentals of academics to include communications, career planning and management, technology, problem solving and critical thinking, health and safety practices, ethics as well as legal responsibilities, leadership development and team work through active participation in the FFA, personal responsibility and flexibility as it applies to specific job skills.

This course carries five units of Life Science credit that meets the high school graduation requirements for each semester completed.

\section{BOARD-ADOPTED TEXTBOOKS}

None

M. SUPPLEMENTARY INSTRUCTIONAL MATERIALS

- Modern Livestock and Poultry Production, $7^{\text {th }}$ Edition, James R. Gillespie

- Various trade magazines and literature

- Various newspapers, magazines and internet research

- Field trips to World Ag Expo and local livestock facilities

- Laboratory manuals and laboratory equipment

- Additional materials that are not limited to, but may include:

- All supplemental material utilized by pet groomers, veterinary assistants, and veterinary clinics including; principle tools used in surgery, dissection, necropsy, and postmortem exams. Grooming sheers, and biohazard waste removal.

- Computer hardware and software, calculators, probe ware.

- Internet access for scientific journals and research information and interactive agriculture web sites.

- Audio / visual materials (Materials used will be those that accompany the text as well as publications by NOVA, Discovery, National Geographic, PBS, etc.)

\section{N. BRIEF OUTLINE OF COURSE CONTENT}

\begin{tabular}{|c|c|c|c|}
\hline Unit & Topic & $\begin{array}{c}\text { Bio/Life } \\
\text { Science } \\
\text { Standards } \\
\text { Addressed }\end{array}$ & $\begin{array}{c}\text { CTE/Ag } \\
\text { Standards } \\
\text { Addressed }\end{array}$ \\
\hline \multirow{1}{1.}{} & Careers in Animal Care & & F1.3-12.4.3, F2.1- \\
& & & 2.7, F2.2-2.5, F2.2- \\
& & & 1.3, F2.2-1.7, F2.2- \\
& & & 2.5, F2.4-1.1, F2.4- \\
& & & 2.3, F3.0, F7.0-7.6, \\
\hline
\end{tabular}




\begin{tabular}{|c|c|c|c|}
\hline & & & $\begin{array}{l}\text { F8.3, F9.1, F9.2, } \\
\text { F10.2, F11.0 }\end{array}$ \\
\hline 2. & $\begin{array}{l}\text { Laws and Animal } \\
\text { Regulations }\end{array}$ & & $\begin{array}{l}\text { D1.4, D9.2, D9.3, } \\
\text { D10.2, D12.0, F1.3- } \\
\text { 12.2.3, F6.1, F8.1, } \\
\text { F8.2, F8.3, F9.4, } \\
\text { F11.0, FD8.3, }\end{array}$ \\
\hline 3. & HACCP Ethics Training & & $\begin{array}{l}\text { D6.5, D9.3, D9.4, } \\
\text { D12.1, F6.1, F6.2, } \\
\text { F9.4, F11.0 }\end{array}$ \\
\hline 4. & $\begin{array}{l}\text { Animal Handling: Restraint, } \\
\text { Transport, Disposal and } \\
\text { Harvest }\end{array}$ & & $\begin{array}{l}\text { D1.3, D1.4, D9.2, } \\
\text { D9.4, D12.1, } \\
\text { D12.2, D12.3, } \\
\text { D12.4, D12.5, } \\
\text { D12.6, F1.3-12.2, } \\
\text { F8.1, F8.2,F11.0, } \\
\text { FD8.1, FD8.2 }\end{array}$ \\
\hline 5. & $\begin{array}{l}\text { Animal Behavior and } \\
\text { Observation }\end{array}$ & BI 1.d, BI 1.a & $\begin{array}{l}\text { D5.1, D9.1, D9.2, } \\
\text { D10.1, D11.3, } \\
\text { F5.1, F5.2, F5.3, } \\
\text { F11.0 }\end{array}$ \\
\hline 6. & $\begin{array}{l}\text { Animal Health: } \\
\text { Management, Prevention, } \\
\text { Housing }\end{array}$ & BI 1.I & $\begin{array}{l}\text { D1.1, D1.2, D3.2, } \\
\text { D6.4, D9.2, D10.1, } \\
\text { D11.3, F2.2-2.3, } \\
\text { F5.1, F5.2, F5.3, } \\
\text { F8.1, F11.0 }\end{array}$ \\
\hline 7. & $\begin{array}{l}\text { Animal Breed } \\
\text { Identification }\end{array}$ & & D5.0 \\
\hline 8. & $\begin{array}{l}\text { Introduction to Small \& } \\
\text { Specialty Animals }\end{array}$ & & $\begin{array}{l}\text { D10.1, D10.2, } \\
\text { D11.1, D11.2, } \\
\text { D11.4, F11.0 }\end{array}$ \\
\hline 9. & $\begin{array}{l}\text { Basic Animal Nutrition: } \\
\text { Ruminant, Monogastric, } \\
\text { Avian, Equine }\end{array}$ & $\begin{array}{l}\text { BI 1.g, BI 1.e, BI 4.e, } \\
\text { BI 4.f, }\end{array}$ & $\begin{array}{l}\text { D2.2, D2.3, D2.4, } \\
\text { D3.1, D9.2, D10.1, } \\
\text { D11.2, D11.3, } \\
\text { F11.0 }\end{array}$ \\
\hline 10. & $\begin{array}{l}\text { Animal } \\
\text { Anatomy/Physiology }\end{array}$ & $\begin{array}{c}\text { BI 1.j, BI 2.a, BI 9.a, } \\
\text { BI 9.b, BI 9.c, BI 9.d, } \\
\text { BI 9.e, BI 9.f, BI 9.g, } \\
\text { BI 9.i, }\end{array}$ & $\begin{array}{l}\text { D2.3, D2.4, D3.1, } \\
\text { D3.2 D4.0, F11.0 }\end{array}$ \\
\hline 11. & $\begin{array}{l}\text { Introduction to Basic Pet } \\
\text { Grooming }\end{array}$ & & $\begin{array}{l}\text { D1.4, F2.4-1.1, } \\
\text { F5.1, F5.2, F5.3, } \\
\text { F.6, F8.2, F8.3, } \\
\text { F9.5, F9.3, F11.0 }\end{array}$ \\
\hline 12. & $\begin{array}{l}\text { Introduction to Diseases } \\
\text { and Control , Non Living } \\
\text { and Living Agents, Spread }\end{array}$ & $\begin{array}{l}\text { BI 1.c, BI 10.a, BI } \\
\text { 10.b, BI 10.c, BI } \\
\text { 10.d }\end{array}$ & $\begin{array}{l}\text { D6.1, D6.2, D6.3, } \\
\text { D6.4, D6.5, D6.6, }\end{array}$ \\
\hline
\end{tabular}




\begin{tabular}{|c|c|c|c|}
\hline & $\begin{array}{l}\text { of Disease, Elimination, } \\
\text { Protection Against, First } \\
\text { and Secondary Lines of } \\
\text { Defense }\end{array}$ & & $\begin{array}{l}\text { D6.7, F2.1-2.3, } \\
\text { F5.1, F11.0 }\end{array}$ \\
\hline 13. & $\begin{array}{l}\text { Common Diseases: } \\
\text { Parasites, Viral, Bacterial, } \\
\text { Fungal, Protozoan, } \\
\text { Zoonotic, Nutritional } \\
\text { disease, Poison, Stress and } \\
\text { Heredity }\end{array}$ & $\begin{array}{l}\text { BI 1.c, BI 10.a, BI } \\
\text { 10.b, BI 10.c, BI } \\
\text { 10.d }\end{array}$ & $\begin{array}{l}\text { D2.2, D6.3, D6.6, } \\
\text { F2.4-1.7, F11.0 }\end{array}$ \\
\hline 14. & $\begin{array}{l}\text { Normal Values: } \\
\text { Temperature, Pulse, } \\
\text { Breathing and Respiration, } \\
\text { Skin, Membranes, } \\
\text { Intestinal Discharge }\end{array}$ & BI 1.j, BI 10.a, & $\begin{array}{l}\text { D3.1, F4.0-4.6, } \\
\text { F6.5, F11.0 }\end{array}$ \\
\hline 15. & $\begin{array}{l}\text { Pharmacology: Route of } \\
\text { Administration, } \\
\text { Measurement, Storage, Size } \\
\text { and Shape of Needles and } \\
\text { Syringes, Common Drugs, } \\
\text { Prescription Requirements }\end{array}$ & BI 5.a & $\begin{array}{l}\text { F1.1-10.0, F1.1- } \\
\text { 13.0, F1.1-15.0, } \\
\text { F1.1-8.0, F4.0-4.6, } \\
\text { F6.2, F6.4, F6.5, } \\
\text { F6.6, F8.1-F8.3, } \\
\text { F11.0 }\end{array}$ \\
\hline 16. & $\begin{array}{l}\text { Emergency Procedures: } \\
\text { First Responders Kit, Splint } \\
\text { techniques, Wound packing }\end{array}$ & & $\begin{array}{l}\text { D1.4, F4.0-4.6, } \\
\text { F6.6, F9.6, F11.0 }\end{array}$ \\
\hline 17. & $\begin{array}{l}\text { Common Surgical } \\
\text { Procedures: Aseptic } \\
\text { technique, Surgical } \\
\text { preparation, Castration, } \\
\text { Abscesses, Dentistry, Suture } \\
\text { technique }\end{array}$ & BI 1.a, BI 10.e & $\begin{array}{l}\text { F4.0-4.6, F6.4, } \\
\text { F6.5, F6.6, F8.1- } \\
\text { F8.3, F11.0 }\end{array}$ \\
\hline 18. & $\begin{array}{l}\text { Laboratory Procedures: } \\
\text { Microscope use, Equipment } \\
\text { Identification, Collecting } \\
\text { and Handling Specimens }\end{array}$ & $\begin{array}{l}\text { BI 1.j, BI 1.c, BI 1.d, } \\
\text { BI 1.e, BI 1.f }\end{array}$ & $\begin{array}{l}\text { D1.4, D4.4, D6.1, } \\
\text { D9.3, F1.2-1.a, } \\
\text { F1.2-1.c, F1.2-1.d, } \\
\text { F1.2-1.j, F1.2-1.m, } \\
\text { F2.1-2.8, F2.2-1.1, } \\
\text { F2.2-1.2, F2.3-1.1, } \\
\text { F2.2-1.2, F2.2-1.3, } \\
\text { F6.4, F6.5, F6.6, }\end{array}$ \\
\hline 19. & $\begin{array}{l}\text { Reproductive Processes: } \\
\text { Gestation, Parturition, } \\
\text { Artificial insemination, } \\
\text { Embryo transfer }\end{array}$ & $\begin{array}{c}\text { BI 2.a, BI 2.b, BI } \\
\text { 2.d, BI 2.e, BI 2.f, BI } \\
\text { 2.g, BI 3.a, BI 3.b, BI } \\
\text { 3.c, BI 3.d }\end{array}$ & $\begin{array}{l}\text { D4.1, D4.2, D4.3, } \\
\text { D4.4, D4.5, D5.1, } \\
\text { D5.2, D5.4 }\end{array}$ \\
\hline
\end{tabular}

\section{O. BEHAVIORAL OBJECTIVES FOR AGRICULTURE 3}

The student will:

1. Identify the attitude and behavior of healthy animals 
2. Recognize the normal skin and mucus membrane color of domestic animals and relate them to specific cases.

3. Monitor temperature, pulse and respiration of animals, and compare the monitored animal to that of the normal animal.

4. Interpret the effects of environmental conditions that relate to temperature, pulse, and respiration.

5. Write an essay on the disease concept of animal health.

6. Design a housing facility for small or large animals that provides for a healthy environment.

7. Demonstrate the need for a balanced diet in animals by balancing a ration.

8. Distinguish between sound and unsound animal disease management techniques.

9. Demonstrate the need for proper sanitation and disinfect ion of an animal's environment by taking bacterial cultures and analyzing the samples

10. Develop and apply a sanitation and disinfection regime for large animals.

11. Identify and analyze the specific structures found in cells of the animal body.

12. Recognize the characteristics of epithelium, connective and muscle tissue by microscopic observation.

13. Differentiate among the functions of the digestive, circulatory, respiratory and excretory systems.

14. Distinguish between the living and non-living causes of disease in animals.

15. Identify the life cycles of bacteria, fungi, viruses, and protozoa's.

16. Classify the various means by which diseases are spread in an environment.

17. Cite the way in which an animal develops resistance and immunity to pathogenic organisms.

18. Contrast the primary and secondary defense mechanism of an animal's body.

19. Identify, by structure, the differences among Diplococcic, Staphylococcus and Streptococcus bacteria.

20. Explain how a change in environmental conditions might alter the growth of a bacterium.

21. Compare and contrast Bovine Mastitis and Blackleg in a written review.

22. Identify by classification Poxviruses, Herepsviruses, Reoviruses, and Corona viruses and determine a common disease caused by each virus.

23. Outline a common method by which a livestock producer could control diseases caused by viruses.

24. Prepare a written report on either Rabies or Canine Distemper. 
25. Recognize the effects that internal and external parasites taking in consideration the parasite's life cycle.

26. Identify by gross observation the physical differences among ticks, fleas, flies, lice and mites.

27. Develop a method for control of common external parasites taking in consideration the parasite's life cycle.

28. Discriminate, by gross examination, the characteristics of Nematodes, Cestodes and Trematodes.

29. Outline a viable program for internal parasites and diseases.

30. Extrapolate upon the relationship of internal parasites and diseases.

31. Explain the importance of proteins, vitamins and minerals as they relate to tissue building' by nutritional experimentation.

32. Formulate a balanced ration that can be fed to either small or large animals.

33. Discriminate between symptoms of a poisoned animal and that of a parasitized animal.

34. Calculate the proper dosage of a medication to be prescribed for a large or small animal.

35. Identify environmental, nutritional and pathogenic stressors that might lower an animal's resistance to disease.

36. Develop a management technique for receiving young transported animals.

37. Recognize the importance that heredity plays in congenital diseases by using mathematical equations.

38. Research the hormone and identify where the hormone is released and its target organ.

39. Research a hormone and explain the effects it has on the animals' body.

40. Dissect the reproductive tract of the cow and be able to identify and explain the function of each part.

41. Be able to remove semen from a semen tank and properly thaw the semen and prepare it for artificial insemination.

\section{G. ASSESSMENT PROCEDURES}

Grading Scale:

$A=90-100 \%$

$\mathrm{B}=80-90 \%$

$C=70-79 \%$

$\mathrm{D}=60-69 \%$

$\mathrm{F}=0-59 \%$

Quarter Grade Determination:

\section{ASSIGNMENT CODE \% WEIGHT}




\begin{tabular}{|l|l|l|}
\hline Assignments & ASMT & $20 \%$ \\
\hline Labs/Activities & LBS & $25 \%$ \\
\hline Tests/Quizzes & TST & $45 \%$ \\
\hline FFA & FFA & $10 \%$ \\
\hline & TOTAL & $\mathbf{1 0 0} \%$ \\
\hline
\end{tabular}

Semester Grades are based on the following:

Quarter $1 \quad 50 \%$

Quarter $2 \quad 50 \%$

** Semester final is a project incorporated in the second quarter grade. 
DPC\#:

Approved by Board:

\section{COURSE OF STUDY}

Ag 4- Ag Sales and Marketing

$11 / 12$

Applied Arts

10

Course Title

Grade Level

Max. Credit

(Title must correlate with Course Code Catalog)

Does this course satisfy a graduation

If so, what subject

area?

Requirement in another subject area?

NO

Prepared by: Amie Mertz

Ridgeview

$3 / 1 / 12$

Date

School

Approval of Site Administrator:

Date

\section{Signature}

\section{A. COURSE INFORMATION}

Grade Level:

Length of Course:

Maximum Credit:

Recommendation for Enrollment:
$11 / 12$

1 year

10 units

Completion of two to three agricultu classes.

\section{B. BRIEF DESCRIPTION OF THE COURSE}

This class is a survey and basic understanding of the business of the agriculture industry with a specific emphasis on agriculture sales, marketing and advertising. It is an introduction to agriculture business and its impact on the agricultural producer, consumer and the food system, and how these 
concepts are applied to create, sell and market agriculture products. The management principles encountered in the day to day operation of an agricultural enterprise are stressed as they relate to the decision making process.

\section{BOARD-ADOPTED TEXTBOOKS \\ None}

\section{SUPPLEMENTARY INSTRUCTIONAL MATERIALS}

Marketing Challenge by the National FFA Association

Introduction to Agribusiness, Delmar Publishing, Ricketts \& Rawlins, 2001

CTAP Portfolio Handbook, 2000

Van Fleet and Peterson. Contemporary Management. 3rd Edition, Houghton

Mifflin Publisher,

ISBN 0-395-69216-4

Rue and Byars. Management, Skills and Application. 6th Edition, Irwin Publisher, ISBN 0-256-110002-6

Agricultural Economics and Agribusiness. 9th edition, Cramer, Gail L., Jensen, Clarence W., Wiley and

Sons, Inc.

Prentice Hall. Agribusiness Management.

Selling: Helping Customers Buy, $3^{\text {rd }}$ edition, Ditzenberger \& Kidney, 1992.

Exploring Farm Cooperatives, Agric. Council of CA 2003

Agribusiness Fundamentals and Applications. $2^{\text {nd }}$ Edition, Delmar Cengage

Learning, 2009

ISBN-13: 978-1-41803231-9

Agribusiness: Management, Marketing, Human Resource Development,

Communication and Technology, Gibson/Usry/Hass/Liles/Moore

Various trade magazines and literature

Various newspapers, magazines and internet research

Field trips to World Ag Expo and local agricultural businesses

Guest speakers from local agricultural businesses and the Farm Bureau

\section{E. BRIEF OUTLINE OF COURSE CONTENT}

1. The role and organization of the Agribusiness

a. Agribusiness's place in California, United States, and the global economy

b. Types of agribusiness

c. The organization of an agribusiness 
d. Managing the agribusiness

2. Models of management, organizations, and work.

a. Survey and exploration of careers in the Ag Business industry

b. Managerial work

c. The agriculture organization

d. Working in the agriculture organization

3. Goals in the agribusiness organization

a. The nature of goals

b. Multiple organizational goals

c. Managerial process of goal setting

d. Evaluation of goal attainment

4. The Decision Maker

a. Image formation

b. Behavioral decision methods

c. Quantitative decision methods

5. Financial Management and Control of Agribusiness

a. Overview of financial statements

b. Controlling and managing the agribusiness

c. Sources of financing

6. Communication in the Agribusiness Organization

a. The communication process

b. Communication and organization structure

c. Communication and the managerial process

7. Leadership

a. The nature of leadership

b. Factors that influence the leadership role

c. The role of the agriculture manager

8. Business Etiquette

a. Professional dress for men and women

b. Business conversation

c. Men and women as colleagues

d. Restaurant and table etiquette

e. Telephone etiquette

9. Marketing and Sales

a. Introduction to selling

b. Preparing to sell

c. Developing sales skills

d. Selling

e. Careers in Selling

f. Advertisements

g. Marketing Plan

1. SWOT Analysis

2. Long Term and Short Term Goals

10. Planning in the Agribusiness

a. A model of planning

b. Designing plans 
c. Organizing for planning

11. Ethics in Agriculture
a. Agriculture values
b. Agriculture ethics
c. Personal values

12. Agriculture Employee Motivation
a. Nature of human motivation
b. Equity in the workplace
c. Labor relations

13. Groups and Teams
a. The nature of groups
b. The character of groups
c. Teams and team building

\section{F. BEHAVIORAL OBJECTIVES FOR (TITLE OF COURSE)}

At this conclusion of this course, the student should be able to:

1. Develop a basic understanding of how economic principles relate to commodity marketing sub sectors in agriculture.

2. Develop a sales presentation.

3. Evaluate a customer complaint and handle it correctly within the company's guidelines - customer relations.

4. Analyze information about a company and its products to take telephone orders.

5. Assess a prospective customer, develop rapport and establish a sale or follow up call customer prospecting.

6. Develop a marketing plan as a team based on a company's current situation and proposed goals.

7. Develop an understanding of the kinds of agricultural business organizations, and the principles and functions involved in their organization and operation

8. Assess people behavior in organizations.

9. Design a model plan to allocate resources for an agribusiness organization using a variety of computer software programs.

10. Compare and contrast the four functions of management and how they relate to the agribusiness organization.

11. Develop an awareness of the basic laws, regulations, and regulatory agencies that interact with the agriculture community.

12. Describe the nature of leadership and the role of the manager as a leader.

13. Solve problems in the areas of personnel, ethics and planning.

\section{Foundation Standards}

\subsection{Mathematics : Specific applications of Algebra I standards (grades eight through twelve): \\ (10.0) Students add, subtract, multiply, and divide monomials and polynomials. Students solve multistep problems, including word problems, by using these techniques.}

(12.0) Students simplify fractions with polynomials in the numerator and denominator by factoring both and reducing them to the lowest terms. 
(13.0) Students add, subtract, multiply, and divide rational expressions and functions. Students solve both computationally and conceptually challenging problems by using these techniques.

(15.0) Students apply algebraic techniques to solve rate problems, work problems, and percent mixture problems.

Specific applications of Geometry standards (grades eight through twelve):

(8.0) Students know, derive, and solve problems involving the perimeter, circumference, area, volume, lateral area, and surface area of common geometric figures.

(10.0) Students compute areas of polygons, including rectangles, scalene triangles, equilateral triangles, rhombi, parallelograms, and trapezoids.

(11.0) Students determine how changes in dimensions affect the perimeter, area, and volume of common geometric figures and solids.

(12.0) Students find and use measures of sides and of interior and exterior angles of triangles and polygons to classify figures and solve problems.

\section{Specific applications of Probability and Statistics standards (grades eight} through twelve):

(8.0) Students organize and describe distributions of data by using a number of different methods, including frequency tables, histograms, standard line and bar graphs, stem-and-leaf displays, scatterplots, and box-and-whisker plots.

1.2 Science: Specific applications of Investigation and Experimentation standards (grades nine through twelve):

(1.a) Select and use appropriate tools and technology (such as computer-linked probes, spreadsheets, and graphing calculators) to perform tests, collect data, analyze relationships, and display data.

(1.c) Identify possible reasons for inconsistent results, such as sources of error or uncontrolled conditions.

(1.d) Formulate explanations by using logic and evidence.

(1.f) Distinguish between hypothesis and theory as scientific terms.

(1.j) Recognize the issues of statistical variability and the need for controlled tests.

(1.l) Analyze situations and solve problems that require combining and applying concepts from more than one area of science.

(1.m) Investigate a science-based societal issue by researching the literature, analyzing data, and communicating the findings. Examples of issues include irradiation of food, cloning of animals by somatic cell nuclear transfer, choice of energy sources, and land and water use decisions in California.

1.3 History-Social Science : Specific applications of Principles of Economics standards (grade twelve):

(12.2) Students analyze the elements of America's market economy in a global setting.

(12.2.2) Discuss the effects of changes in supply and/or demand on the relative scarcity, price, and quantity of particular products.

(12.2.3) Explain the roles of property rights, competition, and profit in a market economy.

(12.2.5) Understand the process by which competition among buyers and sellers determines a market price. 
(12.2.6) Describe the effect of price controls on buyers and sellers.

(12.2.7) Analyze how domestic and international competition in a market economy affects goods and services produced and the quality, quantity, and price of those products.

(12.2.10) Discuss the economic principles that guide the location of agricultural production and industry and the spatial distribution of transportation and retail facilities.

(12.4) Students analyze the elements of the U.S. labor market in a global setting. (12.4.3) Discuss wage differences among jobs and professions, using the laws of demand and supply and the concept of productivity.

2.0 Communications: Students understand the principles of effective oral, written, and multimedia communication in a variety of formats and contexts. (The standards listed below retain in parentheses the numbering as specified in the English- language arts content standards adopted by the State Board of Education.) 2.1 Reading: Specific applications of Reading Comprehension standards (grades nine and ten):

(2.1) Analyze the structure and format of functional workplace documents, including the graphics and headers, and explain how authors use the features to achieve their purposes.

(2.2) Prepare a bibliography of reference materials for a report using a variety of consumer, workplace, and public documents.

(2.3) Generate relevant questions about readings on issues that can be researched.

(2.6) Demonstrate use of sophisticated learning tools by following technical directions (e.g., those found with graphic calculators and specialized software programs and in access guides to World Wide Web sites on the Internet).

(2.7) Critique the logic of functional documents by examining the sequence of information and procedures in anticipation of possible reader misunderstandings.

(2.8) Evaluate the credibility of an author's argument or defense of a claim by critiquing the relationship between generalizations and evidence, the comprehensiveness of evidence, and the way in which the author's intent affects the structure and tone of the text (e.g., in professional journals, editorials, political speeches, primary source material).

Specific applications of Reading Comprehension standards (grades eleven and twelve):

(2.1) Analyze both the features and the rhetorical devices of different types of public documents (e.g., policy statements, speeches, debates, platforms) and the way in which authors use those features and devices.

(2.3) Verify and clarify facts presented in other types of expository texts by using a variety of consumer, workplace, and public documents.

(2.4) Make warranted and reasonable assertions about the author's arguments by using elements of the text to defend and clarify interpretations.

2.2 Writing: Specific applications of Writing Strategies and Applications standards (grades 9-10)

(1.1) Establish a controlling impression or coherent thesis that conveys a clear and distinctive perspective on the subject and maintain a consistent tone and focus throughout the piece of writing. 
(1.2) Use precise language, action verbs, sensory details, appropriate modifiers, and the active rather than the passive voice.

(1.3) Use clear research questions and suitable research methods (e.g., library, electronic media, personal interview) to elicit and present evidence from primary and secondary sources.

(1.5) Synthesize information from multiple sources and identify complexities and discrepancies in the information and the different perspectives found in each medium (e.g., almanacs, microfiche, news sources, in-depth field studies, speeches, journals, technical documents).

(2.3) Write expository compositions, including analytical essays and research reports:

a. Marshal evidence in support of a thesis and related claims, including information on all relevant perspectives.

b. Convey information and ideas from primary and secondary sources accurately and coherently.

c. Make distinctions between the relative value and significance of specific data, facts, and ideas.

d. Include visual aids by employing appropriate technology to organize and record information on charts, maps, and graphs.

e. Anticipate and address readers' potential misunderstandings, biases, and expectations.

f. Use technical terms and notations accurately.

(2.5) Write business letters:

a. Provide clear and purposeful information and address the intended audience appropriately.

b. Use appropriate vocabulary, tone, and style to take into account the nature of the relationship with, and the knowledge and interests of, the recipients.

c. Highlight central ideas or images.

d. Follow a conventional style with page formats, fonts, and spacing that contribute to the documents' readability and impact.

(2.6) Write technical documents (e.g., a manual on rules of behavior for conflict resolution, procedures for conducting a meeting, minutes of a meeting):

a. Report information and convey ideas logically and correctly.

b. Offer detailed and accurate specifications.

c. Include scenarios, definitions, and examples to aid comprehension (e.g., troubleshooting guide).

d. Anticipate readers' problems, mistakes, and misunderstandings.

Specific applications of Writing Strategies and Applications standards (grades eleven and twelve):

(1.3) Structure ideas and arguments in a sustained, persuasive, and sophisticated way and support them with precise and relevant examples.

(1.6) Develop presentations by using clear research questions and creative and critical research strategies (e.g., field studies, oral histories, interviews, experiments, electronic sources).

(1.7) Use systematic strategies to organize and record information (e.g., anecdotal scripting, annotated bibliographies). 
(1.8) Integrate databases, graphics, and spreadsheets into word-processed documents.

(2.5) Write job applications and résumés:

a. Provide clear and purposeful information and address the intended audience appropriately.

b. Use varied levels, patterns, and types of language to achieve intended effects and aid comprehension.

c. Modify the tone to fit the purpose and audience.

d. Follow the conventional style for that type of document (e.g., résumé, memorandum) and use page formats, fonts, and spacing that contribute to the readability and impact of the document.

(2.6) Deliver multimedia presentations:

a. Combine text, images, and sound and draw information from many sources

(e.g., television broadcasts, videos, films, newspapers, magazines, CD-ROMs, the Internet, electronic media-generated images).

b. Select an appropriate medium for each element of the presentation.

c. Use the selected media skillfully, editing appropriately and monitoring for quality.

d. Test the audience's response and revise the presentation accordingly.

2.3 Written and Oral English Language Conventions:-Specific applications of English Language Conventions standards (grades eleven and twelve):

(1.1) Demonstrate control of grammar, diction, and paragraph and sentence structure and an understanding of English usage.

(1.2) Produce legible work that shows accurate spelling and correct punctuation and capitalization.

(1.3) Reflect appropriate manuscript requirements in writing.

2.4 Listening and Speaking: Specific applications of Listening and Speaking Strategies and Applications standards (grades nine and ten):

(1.1) Formulate judgments about the ideas under discussion and support those judgments with convincing evidence.

(1.7) Use props, visual aids, graphs, and electronic media to enhance the appeal and accuracy of presentations.

(2.2) Deliver expository presentations:

a. Marshal evidence in support of a thesis and related claims, including information on all relevant perspectives.

b. Convey information and ideas from primary and secondary sources accurately and coherently.

c. Make distinctions between the relative value and significance of specific data, facts, and ideas.

d. Include visual aids by employing appropriate technology to organize and display information on charts, maps, and graphs.

e. Anticipate and address the listener's potential misunderstandings, biases, and expectations.

f. Use technical terms and notations accurately.

(2.3) Apply appropriate interviewing techniques:

a. Prepare and ask relevant questions. 
b. Make notes of responses.

c. Use language that conveys maturity, sensitivity, and respect.

d. Respond correctly and effectively to questions.

e. Demonstrate knowledge of the subject or organization.

f. Compile and report responses.

g. Evaluate the effectiveness of the interview.

\section{Specific applications of Listening and Speaking Strategies and Applications} standards (grades 11-12)

(1.8) Use effective and interesting language, including:

a. Informal expressions for effect

b. Standard American English for clarity

c. Technical language for specificity

(1.14) Analyze the techniques used in media messages for a particular audience and evaluate their effectiveness (e.g., Orson Welles' radio broadcast "War of the Worlds").

(2.4) Deliver multimedia presentations:

a. Combine text, images, and sound by incorporating information from a wide range of media, including films, newspapers, magazines, CD-ROMs, online information, television, videos, and electronic media-generated images.

b. Select an appropriate medium for each element of the presentation.

c. Use the selected media skillfully, editing appropriately and monitoring for quality.

d. Test the audience's response and revise the presentation accordingly

\subsection{Career Planning and Management}

Students understand how to make effective decisions, use career information, and manage personal career plans:

3.1 Know the personal qualifications, interests, aptitudes, information, and skills necessary to succeed in careers.

3.2 Understand the scope of career opportunities and know the requirements for education, training, and licensure.

3.3 Develop a career plan that is designed to reflect career interests, pathways, and postsecondary options.

3.4 Understand the role and function of professional organizations, industry associations, and organized labor in a productive society.

3.5 Understand the past, present, and future trends that affect careers, such as technological developments and societal trends, and the resulting need for lifelong learning.

3.6 Know important strategies for self-promotion in the hiring process, such as job applications, résumé writing, interviewing skills, and preparation of a portfolio.

4.0 Technology: Students know how to use contemporary and emerging technological resources in diverse and changing personal, community, and workplace environments:

4.1 Understand past, present, and future technological advances as they relate to a chosen pathway. 
4.2 Understand the use of technological resources to gain access to, manipulate, and produce information, products, and services.

4.3 Understand the influence of current and emerging technology on selected segments of the economy.

4.4 Understand geographic information systems (G.I.S.).

4.5 Determine the validity of the content and evaluate the authenticity, reliability, and bias of electronic and other resources.

4.6 Differentiate among, select, and apply appropriate tools and technology.

5.0 Problem Solving and Critical Thinking: Students understand how to create alternative solutions by using critical and creative thinking skills, such as logical reasoning, analytical thinking, and problem-solving techniques:

5.1 Apply appropriate problem-solving strategies and critical thinking skills to work-related issues and tasks.

5.2 Understand the systematic problem-solving models that incorporate input, process, outcome, and feedback components.

5.3 Use critical thinking skills to make informed decisions and solve problems.

6.0 Health and Safety: Students understand health and safety policies, procedures, regulations, and practices, including the use of equipment and handling of hazardous materials:

6.1 Know policies, procedures, and regulations regarding health and safety in the workplace, including employers' and employees' responsibilities.

6.2 Understand critical elements of health and safety practices related to storing, cleaning, and maintaining tools, equipment, and supplies.

6.3 Understand how to locate important information on a material safety data sheet. 6.4 Maintain safe and healthful working conditions.

6.5 Use tools and machines safely and appropriately.

6.6 Know how to both prevent and respond to accidents in the agricultural industry.

7.0 Responsibility and Flexibility: Students know the behaviors associated with

the demonstration of responsibility and flexibility in personal, workplace, and community settings:

7.1 Understand the qualities and behaviors that constitute a positive and professional work demeanor.

7.2 Understand the importance of accountability and responsibility in fulfilling personal, community, and workplace roles.

7.3 Understand the need to adapt to varied roles and responsibilities.

7.4 Understand that individual actions can affect the larger community.

7.5 Understand the importance of time management to fulfill responsibilities.

7.6 Know how to apply high-quality craftsmanship to a product or presentation and continually refine and perfect it.

8.0 Ethics and Legal Responsibilities: Students understand professional, ethical, and legal behavior consistent with applicable laws, regulations, and organizational norms:

8.1 Know the major local, district, state, and federal regulatory agencies and entities that affect the industry and how they enforce laws and regulations.

8.2 Understand the concept and application of ethical and legal behavior consistent with workplace standards. 
8.3 Understand the role of personal integrity and ethical behavior in the workplace. 8.4 Understand how to access, analyze, and implement quality assurance information.

9.0 Leadership and Teamwork : Students understand effective leadership styles, key concepts of group dynamics, team and individual decision making, the benefits of workforce diversity, and conflict resolution:

9.1 Understand the characteristics and benefits of teamwork, leadership, and citizenship in the school, community, and workplace settings.

9.2 Understand the ways in which preprofessional associations, such as the Future Farmers of America (FFA), and competitive career development activities enhance academic skills, promote career choices, and contribute to employability.

9.3 Understand how to organize and structure work individually and in teams for effective performance and the attainment of goals.

9.4 Know multiple approaches to conflict resolution and their appropriateness for a variety of situations in the workplace.

9.5 Understand how to interact with others in ways that demonstrate respect for individual and cultural differences and for the attitudes and feelings of others. 9.6 Understand leadership, cooperation, collaboration, and effective decisionmaking skills applied in group or team activities, including the student organization.

10.0 Technical Knowledge and Skills : Students understand the essential knowledge and skills common to all pathways in the Agriculture and Natural Resources sector:

10.1 Understand the aims, purposes, history, and structure of the FFA student organization, and know the opportunities it makes available.

10.2 Manage and actively engage in a career-related, supervised agricultural experience.

10.3 Understand the importance of maintaining and completing the California Agricultural Record Book.

10.4 Maintain and troubleshoot equipment used in the agricultural industry. 11.0 Demonstration and Application : Students demonstrate and apply the concepts contained in the foundation and pathway standards.

Agricultural Business Pathway: In the Agricultural Business Pathway, students learn about agricultural business operation and management. Topics include accounting, finance, economics, business organization, marketing, and sales. A1.0 Students understand decision-making processes within the American free enterprise system:

A1.1 Differentiate among the components of the American free enterprise system and other forms of economic systems.

A1.2 Distinguish among the main characteristics of individual proprietorships, partnerships, corporations, and cooperatives.

A1.3 Understand the advantages and disadvantages of the four types of business ownership.

A1.4 Analyze appropriate decision-making tools and financial records to make key management decisions. 
A1.5 Analyze physical production relationships to determine optimum use levels.

A1.6 Understand how to calculate the fixed and variable costs associated with the production of agricultural products and determine the output level that will yield maximum profit.

A2.0 Students understand the fundamental economic principles of agribusiness and agricultural production:

A2.1 Understand how basic economic factors affect agricultural production and agribusiness management decisions.

A2.2 Know basic agricultural economic terminology.

A2.3 Understand the law of supply and demand as it effects price determination. A2.4 Analyze how agriculture uses scarce resources to meet the needs and demands of its consumers.

A2.5 Differentiate between elastic and inelastic supply and demand.

A2.6 Understand the law of diminishing returns and its impact on agricultural production.

A3.0 Students understand the role of credit in agribusiness and agricultural production:

A3.1 Analyze the factors that determine the cost of credit in order to select optimum credit sources (e.g., the advantages and disadvantages of borrowing from the various types of credit providers and sources for short-, intermediate-, and long-term credit).

A3.2 Know the criteria lenders use to evaluate repayment capacity.

A3.3 Analyze balance sheets and cash-flow statements to determine the ability to repay loans.

A4.0 Students understand proper accounting principles and procedures used in business management and tax planning:

A4.1 Understand the differences between cash and accrual accounting systems.

A4.2 Understand the use and importance of budgets, income statements, balance sheets, and financial statements.

A4.3 Understand the basis of taxation within the tax system and its impact on the economy, including the role of taxes in agribusiness.

A4.4 Analyze the role of depreciation and purchasing in tax planning and liability.

A4.5 Understand how to determine property values and how to complete a depreciation schedule.

A4.6 Understand how to determine the tax obligations for an agribusiness.

A5.0 Students understand basic risk management principles and their impact on economic viability:

A5.1 Understand environmental responsibility and its impact on agribusiness.

A5.2 Understand the concept of liability and the economic impact of being held liable.

A5.3 Understand the concept and process of risk management, including the use of risk management tools such as insurance.

A5.4 Understand how recordkeeping, farm plans, and an analysis of best practices affect risk management decisions. 
A5.5 Understand the role of contingency plans in risk management.

A6.0 Students understand the role and value of agricultural organizations:

A6.1 Understand the benefits of private, public, and governmental organizations, including the value and impact of cooperatives.

A6.2 Understand how participation within organizations would be beneficial in supporting various agricultural operations.

A6.3 Understand how to identify and electronically access public and private agricultural organizations.

A7.0 Students understand agricultural marketing systems:

A7.1 Understand how marketing functions in a free market society.

A7.2 Understand the advantages and disadvantages of the various marketing options for agricultural products and services.

A7.3 Understand how the law of comparative advantage affects agricultural production.

A7.4 Understand the impact of advertising and promotion on the marketing of agricultural products and services.

A7.5 Understand how promotion trends for agricultural products influence individuals.

A7.6 Understand how to develop a marketing plan for an agricultural product or service.

A8.0 Students understand the sales of agricultural products and services:

A8.1 Determine the most effective methods for assessing customer needs and wants.

A8.2 Understand the stages in making a successful sale and the various techniques used to approach potential customers and overcome their objections.

A8.3 Examine the physiological and psychological factors that influence motivation to purchase, including the fundamental steps in making a purchase.

A9.0 Students understand local, national, and international agricultural markets and how trade affects the economy:

A9.1 Understand how the importance of agricultural imports and exports affects state and national economies.

A9.2 Know how governmental, economic, and cultural factors affect international trade.

A9.3 Compare and contrast United States trade policies with those of other important trading partners.

A9.4 Understand how biotechnology affects trade and global economies.

A9.5 Understand how different cultural values affect agricultural production and marketing.

A9.6 Understand how negotiations and bargaining agreements affect trade agreements.

A9.7 Analyze agricultural marketing strategies in other parts of the world.

\section{G. ASSESSMENT PROCEDURES}

Homework/Class work 
Supervised Agriculture Experience Program $\quad 15 \%$

FFA - Leadership $20 \%$

Quizzes \& Tests $25 \%$

Participation $15 \%$

SEMESTER BREAKDOWN:

Semester $1=50 \%$

Semester $2=50 \%$

** Semester final is a project incorporated in the second quarter grade.

BAKERSFIELD ESLRs MET BY THIS COURSE: $\quad$ 1a, b, c, 2a, b, c, d, e, f, 3a, b, c, and $\mathrm{d}$. 
Supporting Completion Materials D: Daily Grade Sheets

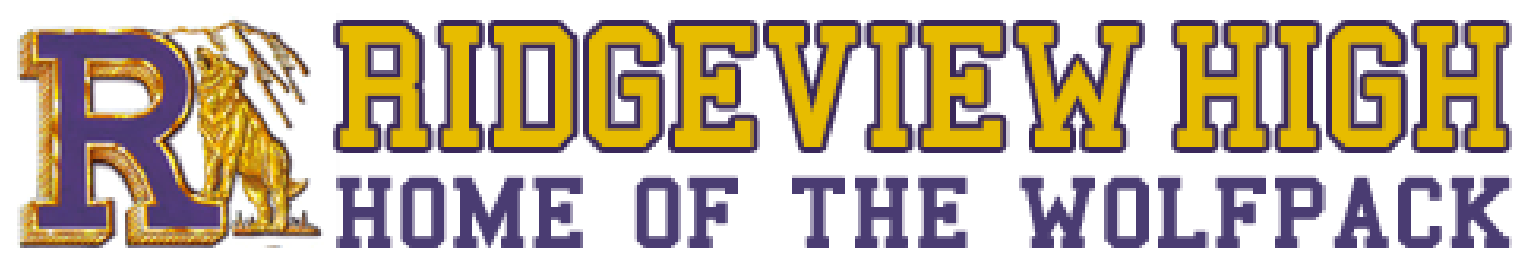




\section{Daily Grade Sheets}

Grades are entered into a district program, Synergy. They are accumulated each quarter and semester according to department policies. Attached are examples of the semester 2 grade calculations for each class.

Supporting Completion Material Attached 
(S2) Potterton, J AGSLES/MKTNG T/A (1) SEC:5811-1-0002

$6 / 30 / 2015$

Page 1 of 1

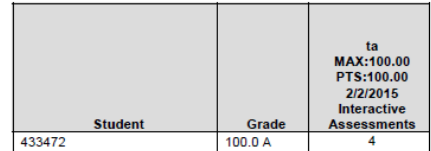

(S2) Potterton, J AGSLES/MKTNG(1) SEC:5811-1-0002 Page 1 of 1

$6 / 30 / 2015$

\begin{tabular}{|c|c|c|c|c|c|c|c|c|}
\hline Student & Grade & $\begin{array}{c}\text { Q3 } \\
\text { MAX:100.00 } \\
\text { PTS: } 40.00 \\
\text { 5/1/12015 } \\
\text { Assessment }\end{array}$ & $\begin{array}{c}\text { Q4 } \\
\text { MAX:100.00 } \\
\text { PTS:40.00 } \\
\text { 5S/1/2015 } \\
\text { Assessment }\end{array}$ & $\begin{array}{c}\text { final } \\
\text { MAX:100.00 } \\
\text { PTS:10.00 } \\
\text { 5/1/12015 } \\
\text { Assessment }\end{array}$ & $\begin{array}{c}\text { SAE } \\
\text { MAX:100.00 } \\
\text { PTS:5.00 } \\
\text { 5T/1/2015 } \\
\text { Assessment }\end{array}$ & $\begin{array}{c}\text { FFA } \\
\text { MAX:100.00 } \\
\text { PTS:5.00 } \\
\text { 5T/112015 } \\
\text { Assessment }\end{array}$ & $\begin{array}{c}\text { XC: } \\
\text { extra FFA } \\
\text { points } \\
\text { MAX:100.00 } \\
\text { PS: } 0.00 \\
\text { 5/1/2015 } \\
\text { Assessment }\end{array}$ & $\begin{array}{c}\text { XC: } \\
\text { more FFA } \\
\text { points } \\
\text { MAX:100.00 } \\
\text { PTS: } 0.00 \\
\text { 5/20/2015 } \\
\text { Assessment }\end{array}$ \\
\hline 451655 & $71.0 \mathrm{C}$ & 82.4 & 66 & 50 & 85 & 47.5 & & \\
\hline 454196 & $51.9 \mathrm{~F}$ & 57 & 52.8 & 50 & 0 & 60 & & \\
\hline 432809 & $84.8 \mathrm{~B}$ & 94.3 & 91.5 & 50 & 0 & 100 & 50 & \\
\hline 454311 & $79.6 \mathrm{C}$ & 72.8 & 77.5 & 90 & 78.5 & 100 & 152.5 & \\
\hline 432860 & $68.6 \mathrm{D}$ & 72.4 & 64.8 & 50 & 76 & 97.5 & & \\
\hline 432865 & $88.7 \mathrm{~B}$ & 97.1 & 92.8 & 75 & 0 & 100 & 27.5 & \\
\hline 432964 & $96.7 \mathrm{~A}$ & 86 & 103 & 95 & 87.5 & 100 & 200 & 22.5 \\
\hline 454423 & $79.6 \mathrm{C}$ & 84 & 76.7 & 85 & 0 & 100 & 180 & \\
\hline 454432 & $77.6 \mathrm{C}$ & 81.7 & 71 & 85 & 92.5 & 67.5 & & \\
\hline 454500 & $76.7 \mathrm{C}$ & 76.7 & 68 & 90 & 90 & 100 & 27.5 & \\
\hline 454525 & $62.7 \mathrm{D}$ & 77.6 & 66.7 & 50 & 0 & 0 & & \\
\hline 464129 & $72.1 \mathrm{C}$ & 67.2 & 77.5 & 90 & 0 & 100 & 22.5 & \\
\hline 454611 & $85.8 \mathrm{~B}$ & 81.5 & 82.3 & 85 & 94 & 100 & 200 & 12.5 \\
\hline 454678 & $82.3 \mathrm{~B}$ & 88.1 & 74.8 & 80 & 90 & 92.5 & & \\
\hline 451888 & $84.8 \mathrm{~B}$ & 83.1 & 86.8 & 95 & 94.5 & 52.5 & & \\
\hline 433300 & $74.0 \mathrm{C}$ & 67.9 & 69.8 & 90 & 90 & 100 & 45 & \\
\hline 454768 & $87.7 \mathrm{~B}$ & 80.8 & 88.3 & 90 & 95.5 & 100 & 127.5 & \\
\hline 454977 & $69.3 \mathrm{D}$ & 72.4 & 64 & 85 & 0 & 125 & & \\
\hline 455026 & $50.6 \mathrm{~F}$ & 65.3 & 46.2 & 50 & 0 & 20 & & \\
\hline 433576 & $82.7 \mathrm{~B}$ & 69.2 & 91.2 & 95 & 45 & 100 & 177.5 & \\
\hline 463271 & $79.2 \mathrm{C}$ & 70.5 & 78.7 & 95 & 0 & 200 & 5 & \\
\hline 455129 & $51.6 \mathrm{~F}$ & 52.7 & 63.8 & 50 & 0 & 0 & & \\
\hline 502432 & $92.3 \mathrm{~A}$ & 82.9 & 104.2 & 95 & 72.5 & 87.5 & & \\
\hline 433756 & $72.2 \mathrm{C}$ & 64.8 & 76.3 & 75 & 45 & 100 & 102.5 & \\
\hline 455399 & $74.9 \mathrm{C}$ & 76 & 77.5 & 85 & 70 & 30 & & \\
\hline 455438 & $70.2 \mathrm{C}$ & 78.1 & 74.5 & 50 & 0 & 82.5 & & \\
\hline 455594 & $79.7 \mathrm{C}$ & 77 & 67.3 & 95 & 96.5 & 152.5 & & \\
\hline 451606 & $72.8 \mathrm{C}$ & 69 & 73.3 & 80 & 82.5 & 75 & & \\
\hline 435004 & $94.7 \mathrm{~A}$ & 87.5 & 84.3 & 95 & 87.5 & 200 & 200 & 11.25 \\
\hline 455743 & $67.2 \mathrm{D}$ & 74.4 & 71.3 & 85 & 0 & 7.5 & & \\
\hline 434737 & $100.5 \mathrm{~A}$ & 92.2 & 99 & 95 & 95 & 195 & & \\
\hline 434369 & $80.1 \mathrm{~B}$ & 80.2 & 87.7 & 95 & 61 & 7.5 & & \\
\hline 434371 & $73.2 \mathrm{C}$ & $\begin{array}{ll}76.3 \\
\end{array}$ & 71.2 & 75 & 41 & 92.5 & & \\
\hline 438648 & $83.0 \mathrm{~B}$ & 77.2 & 76 & 75 & 83 & 200 & 7.5 & \\
\hline
\end{tabular}




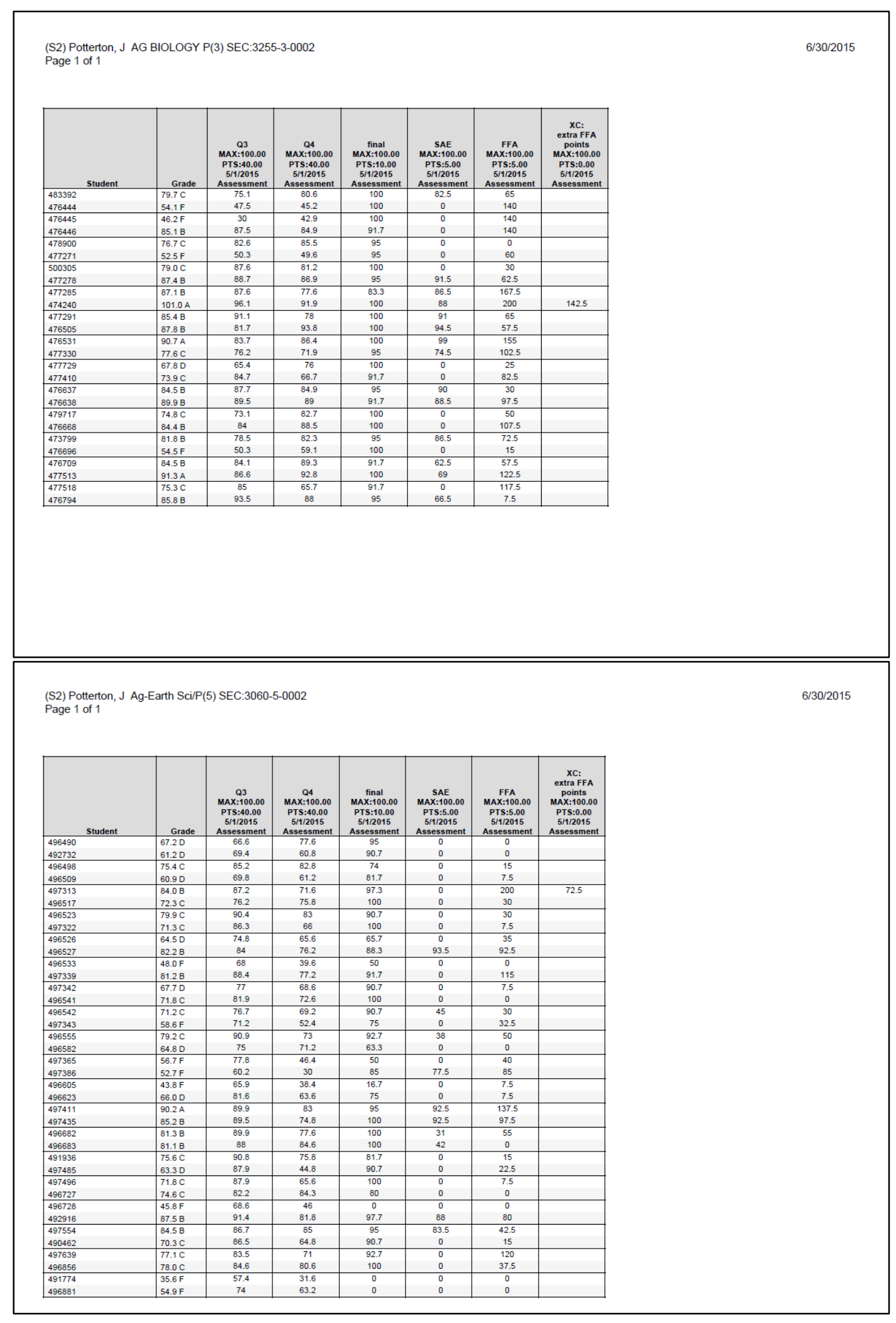




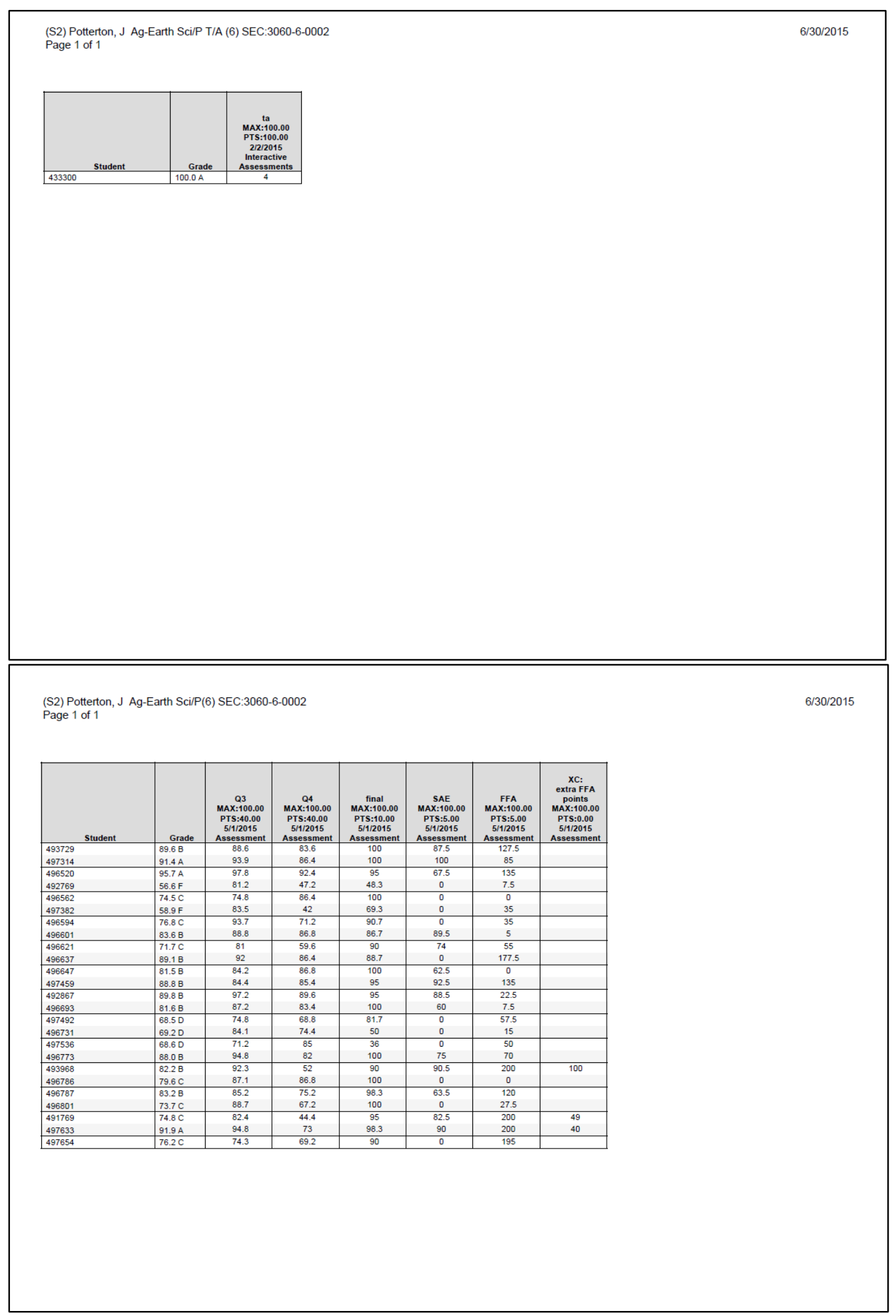




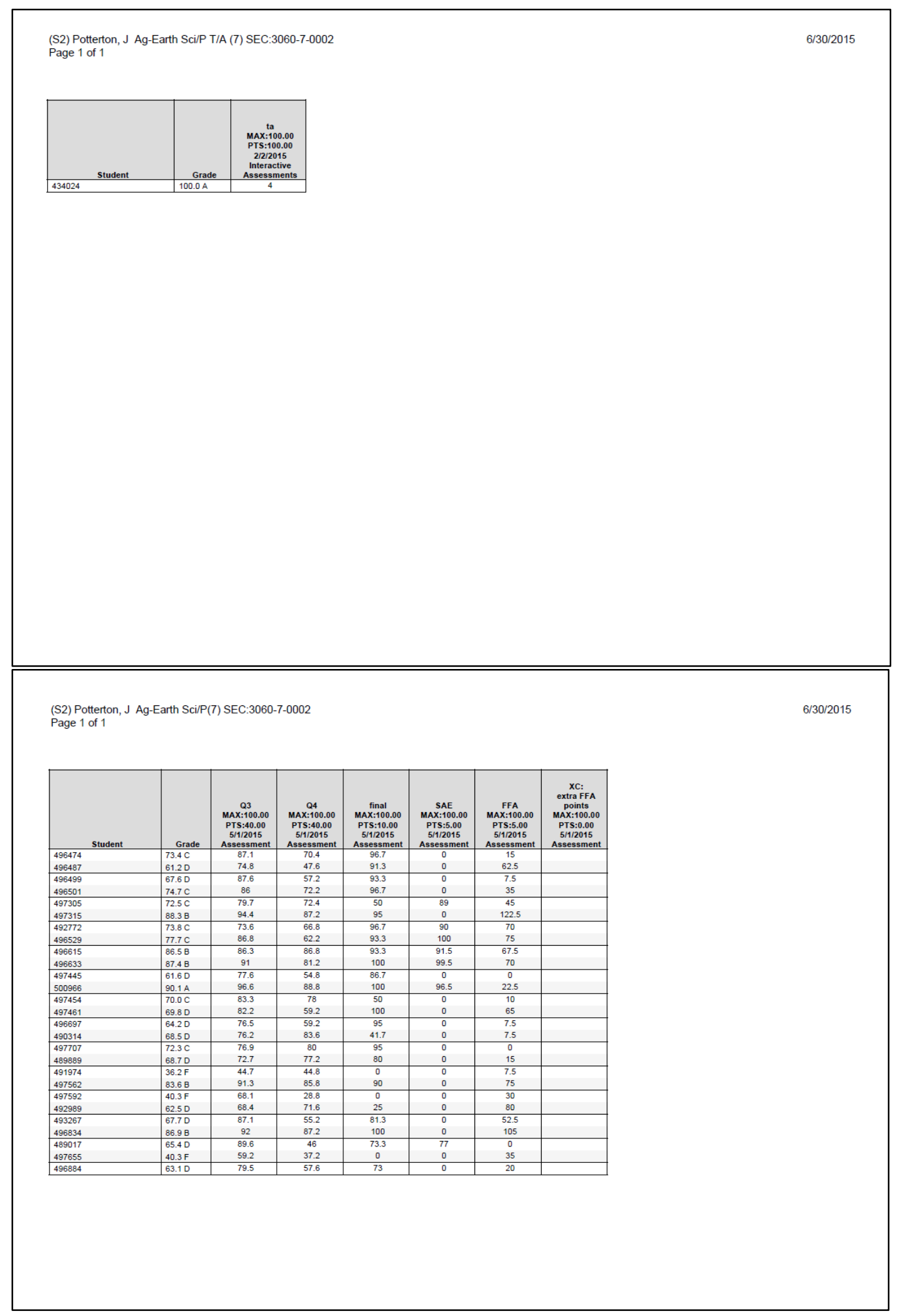


Supporting Completion

Materials E: SAE

Supervision Form

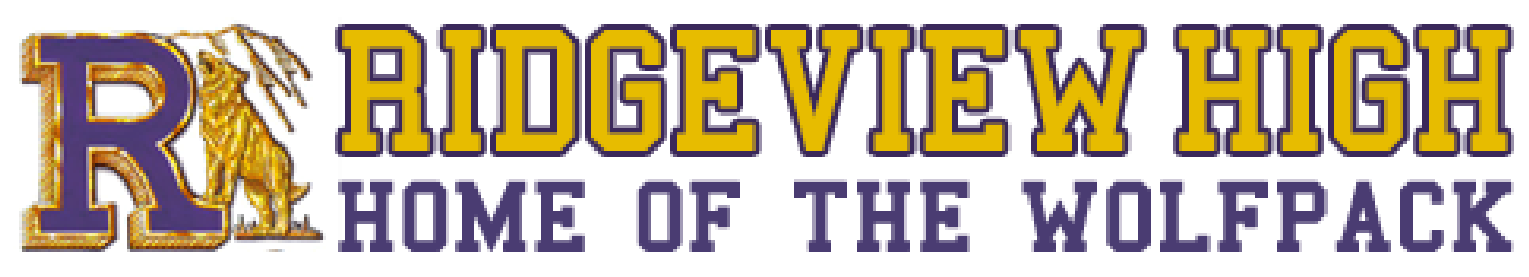




\section{E. SAE Supervision Form}

Project supervision forms are provided as a record between the advisor and student when a project visit occurs. The form is signed at the time of a project visit and a copy is provided to the student while the advisor keeps another copy and kept in the files for the project. The form is essential as a record of instruction to the exhibitor. When I started a similar, but different form was already available, so I am using both forms at this time until the old ones are gone.

Supporting Completion Material Attached 


\section{RIDGEVIEW HIGH SCHOOL AGRICLLTURE DEPARTMENT}

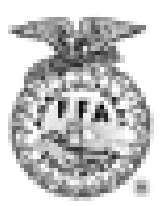

RIDGEVIEW FFA CHAPTER

\section{PROJECT VISIT REPORT}

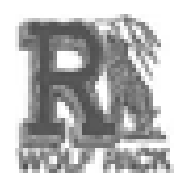

Student Name: Date:

Visiting Advisor: Location:

Species of project:

Project weight[s]: ADG:

Current feeding program:

Medications:

\begin{tabular}{|c|c|c|c|c|c|c|}
\hline Dete & $\begin{array}{l}\text { Animbil } \\
\text { Trested }\end{array}$ & Medicstion & $\begin{array}{c}\text { Amount } \\
\text { Ghen }\end{array}$ & Withdrewel & $\begin{array}{l}\text { Withdressil } \\
\text { End Date }\end{array}$ & 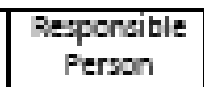 \\
\hline & & & & & & \\
\hline & & & & & & \\
\hline
\end{tabular}

General Comments/Recommendations:

Student Signature:

Advisor signature:

Next Project Visit/showmanship Practice: 


\section{Ridgeview High School Agriculture Department}

Student Name:

Viating Teacher
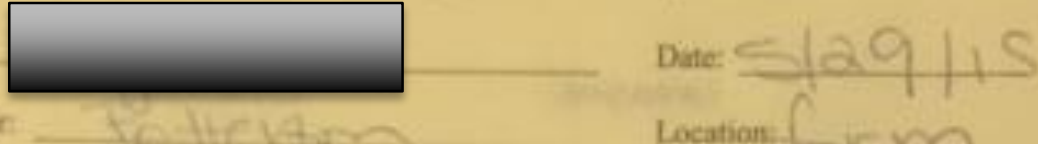

Location:

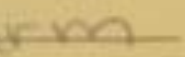

Reserd Book Present and Updated?

If not, *f warning

\section{Proinct Vialifior}

\begin{tabular}{|l|l|l|}
\hline Pig & Dairy Heifer \\
\hline Sheep & & Horse \\
\hline Geat & & Other: \\
\hline Rabhit & & \\
\hline
\end{tabular}

Weights for fair animale Animal ID.

Weight on last visit:
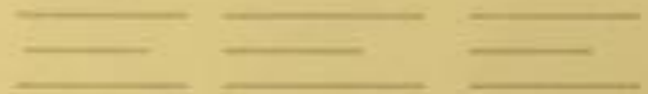

Number of Days aince last visit

Rate of Caise

the day

Itwilay

Ibelday

Boslay

Nimber of days to finir:

Projected Weight Gain

Projected Wt \& Falr:

General Froject Comuments:

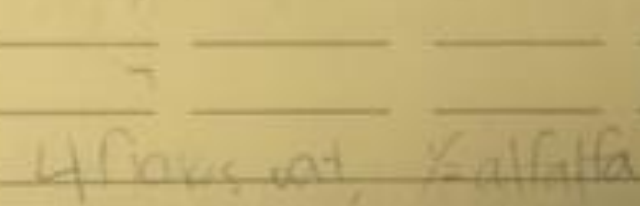

Recounmendatives:

27

ximetion

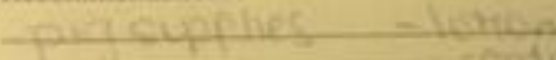

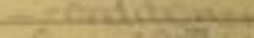

rannitikno

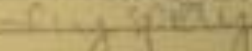

Fair requirements to still meet:

Signed Acknowledjement Sheet

Parent/Fahibiter Meeting

Entries tumed into livestock office Other:
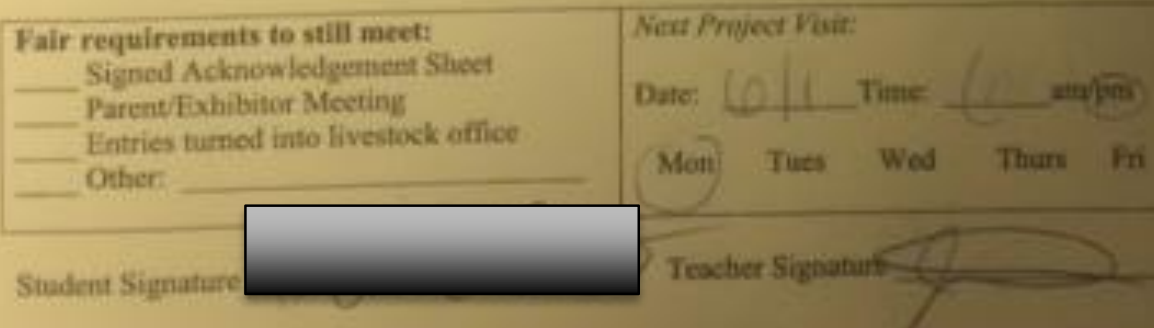


\section{Ridgeview High School Agriculture Department}

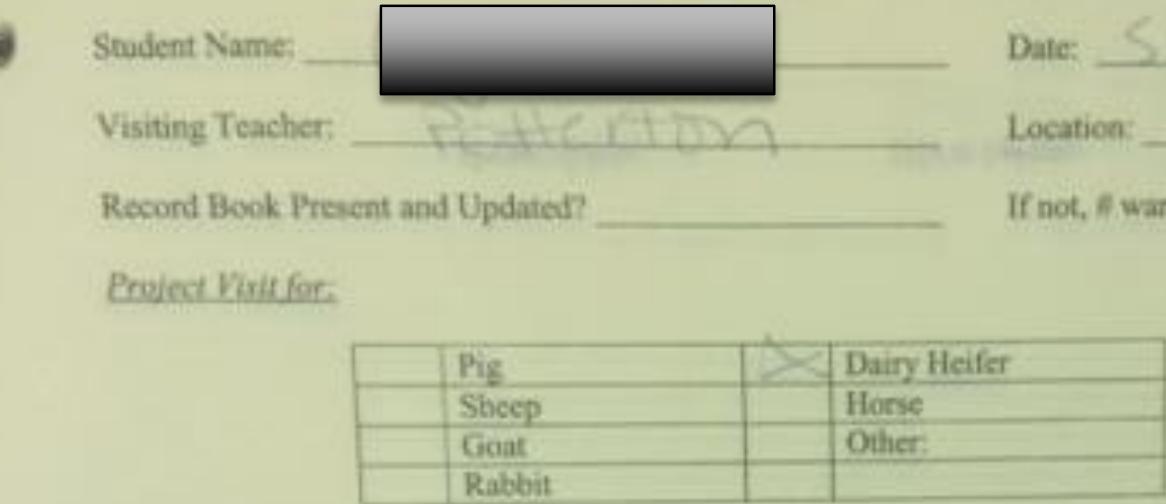

Weighus for fair animals: Anmial ID:

Weight on last vinit:
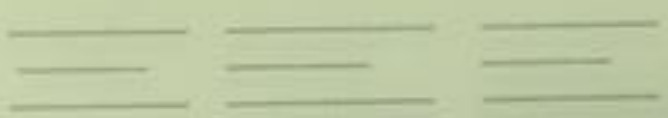

Number of Days since last visit:

Rate of Gain:

losiday

thsiday

fbolday

tosiday

Number of dins to foir:

\section{Projected Weight Gain:}

Projected Wt \& Fair:

General Project Comments:

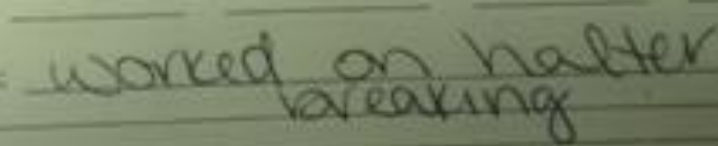

\section{Recommendations:}

\section{Fair reauirements to still mext:} Signed Acknow lodgernent Shect Parent Exhibitor Mecting linaries turnod into livastodk offlce Osher: Student Siguature

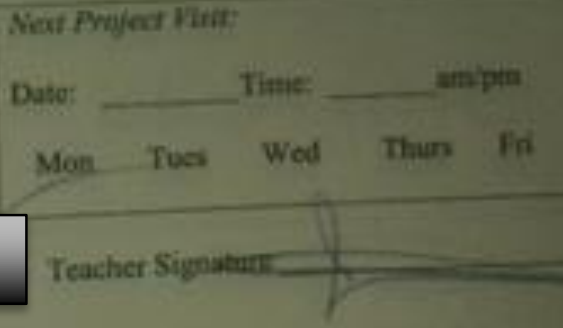




\section{Ridgeview High School Agriculture Department}
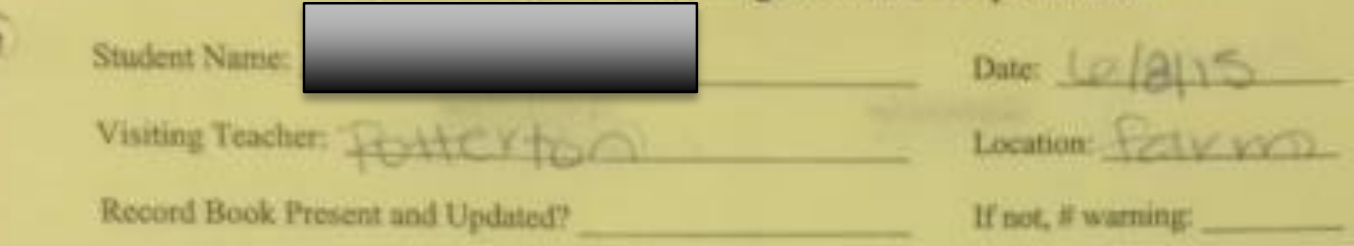

\section{Projet Fisitfor:}

\begin{tabular}{|l|l|l|l|}
\hline Pig & Dairy Heifer \\
\hline Sheep & & Horse \\
\hline Goat & & Other: \\
\hline Rabbit & & \\
\hline
\end{tabular}

Weights for fair animals:

Animal ID

Weight on last visit
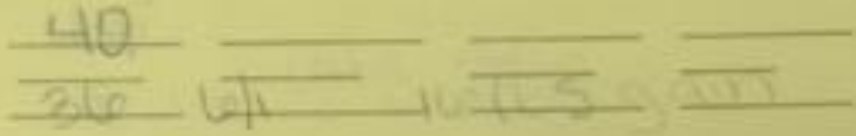

Nimber of Days since last visit:

Rate of Gain:

thaiday

Jowlay

Disay

tbolday

Number af dios bo fair:

Projected Welght Gain:

Projected Wt it Fair:

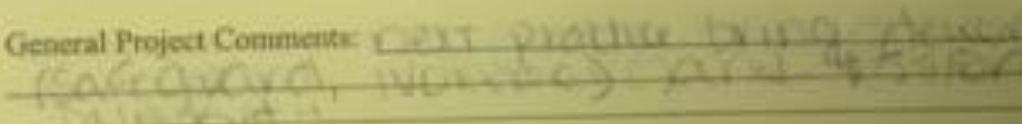

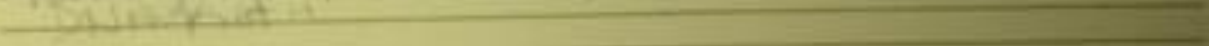

\section{Recommendations:}

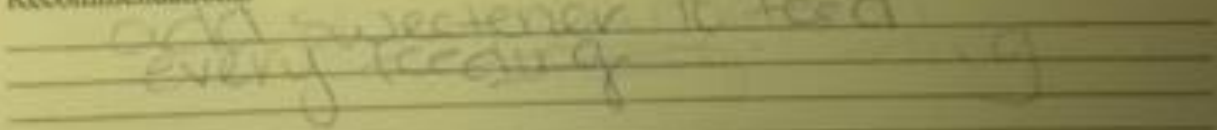

Fair requirements to still meet: Signed Acknow ledgement Sbect Parent/Exhibitor Mcetiny Patries tamed isto Lvestock office Other:

Student Sigmaturt

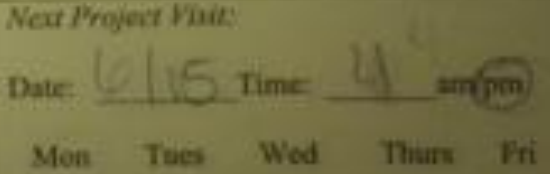

Teacher Sigrititir: 


\section{Ridgeview High School Agriculture Department}
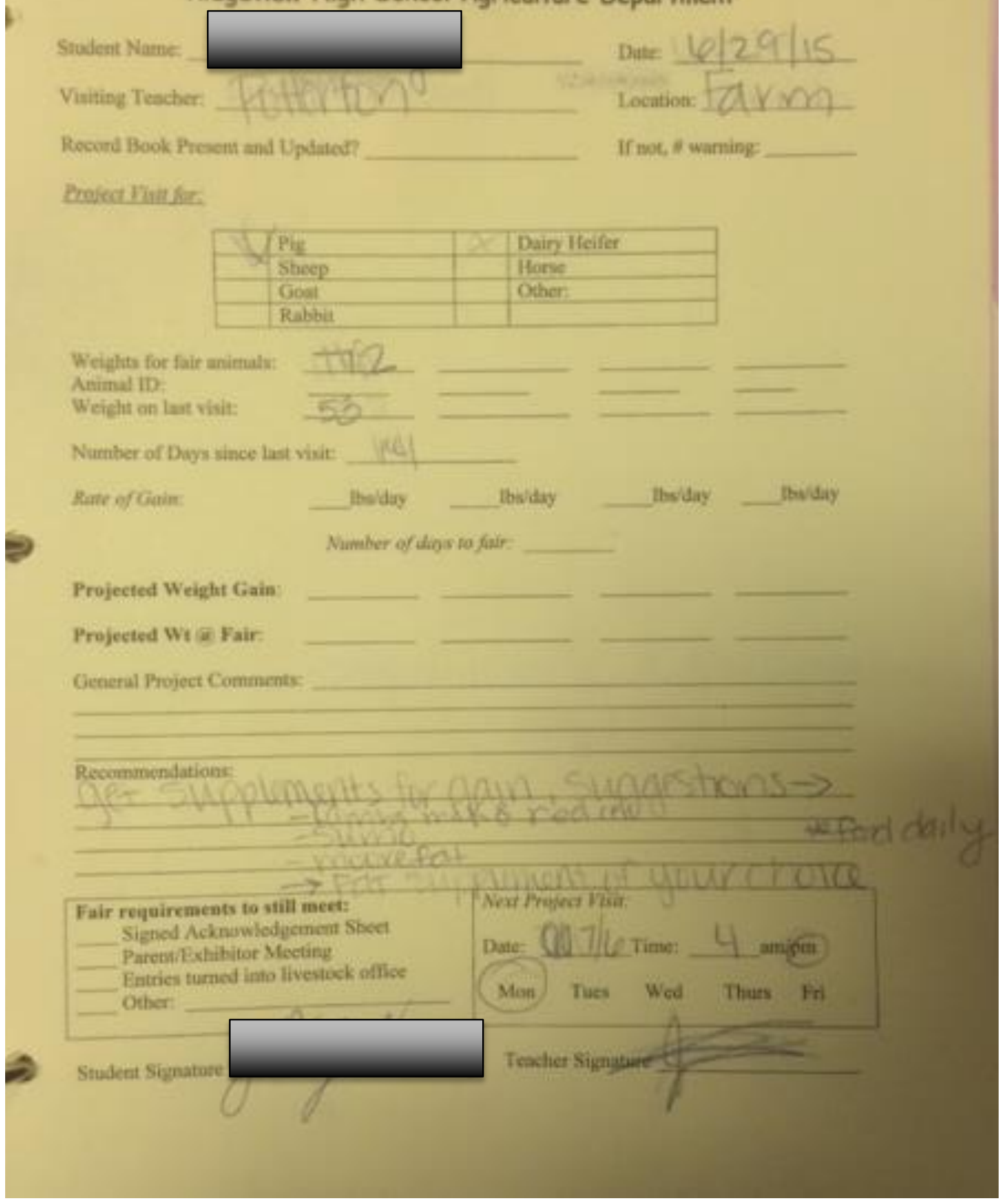


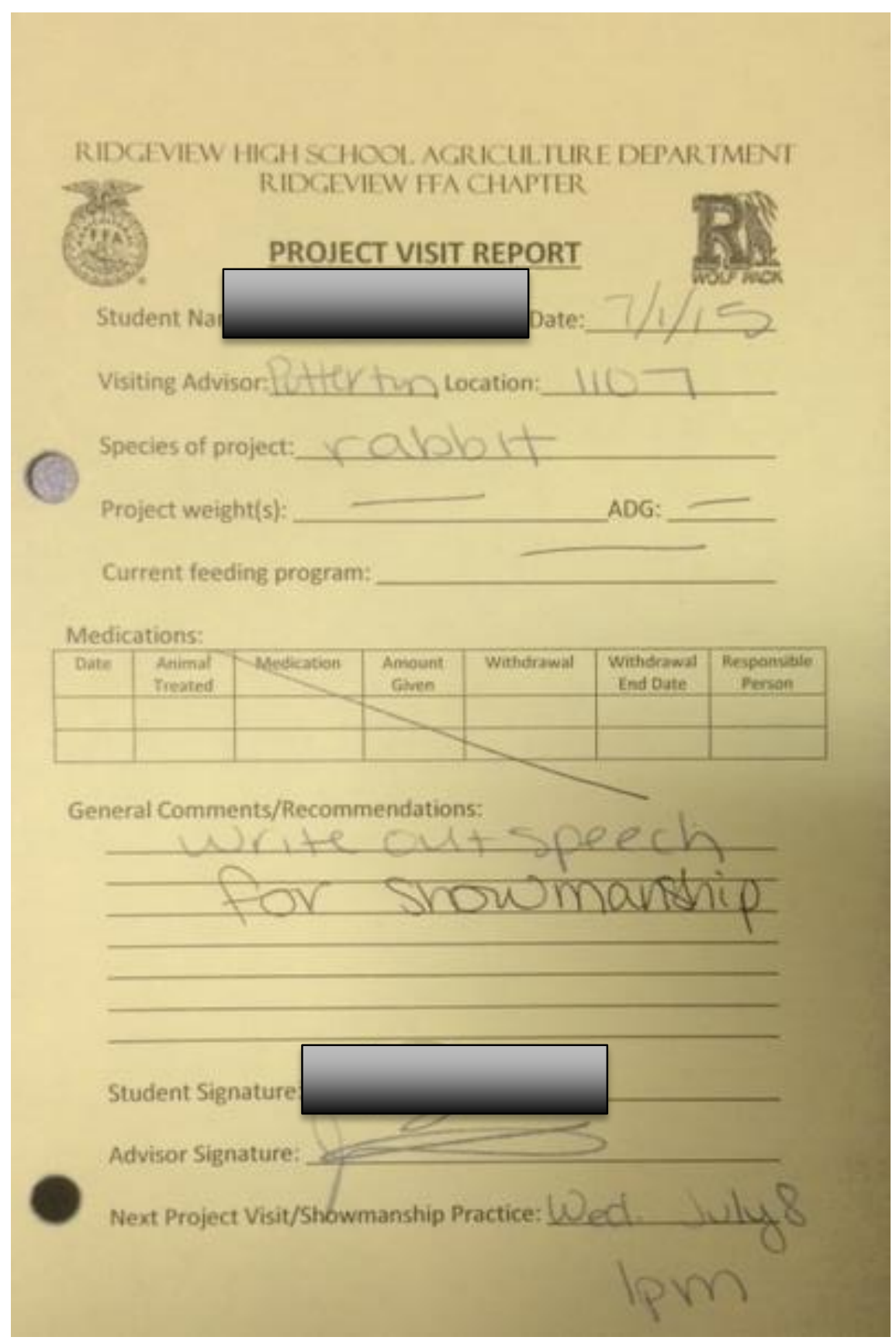


RIDGEVIEW HIGHSCHOOL. AGRICULTURE DEPARTMENT (t) RIDGEVIEW HACHATTER

\section{PROJECT VISIT REPORT}

Student Name

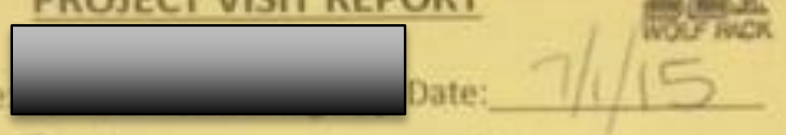

Visiting Advisory Her tun Location:

species of project: CWMCK\&V

Project weight(s):

ADG:

Current feeding program:

Medications:

\begin{tabular}{|c|c|c|c|c|c|c|}
\hline Date & $\begin{array}{l}\text { Animal } \\
\text { Trated }\end{array}$ & Muefication & $\begin{array}{c}\text { Amount } \\
\text { Given }\end{array}$ & Withdeand & $\begin{array}{l}\text { Wahdrowat } \\
\text { Enst Date }\end{array}$ & $\begin{array}{l}\text { Respomible } \\
\text { Perion }\end{array}$ \\
\hline & & & 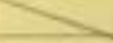 & & & \\
\hline & & & & & & \\
\hline
\end{tabular}

General Comments/Recommendations:

Wr tee Dust suesed for showmanship

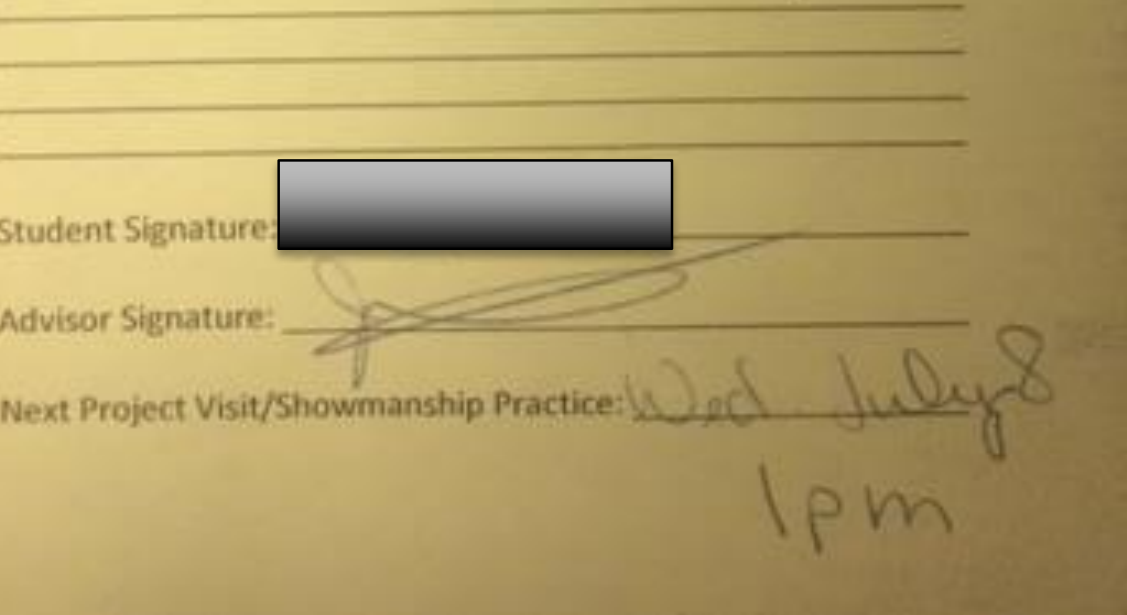




\section{Ridgeview High School Agriculture Department}

Student Name:

Visiting Teacher

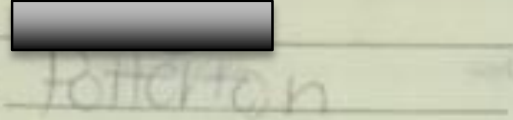

Recond Book Present and Updated?

Date:

Location:

If not, sf waming:

\section{Proiect Visit for}

\begin{tabular}{|l|l|l|}
\hline Pig & & Dairy Heifer \\
\hline Sheep & & Hone \\
\hline Gout & & Other \\
\hline Rubbit & & \\
\hline
\end{tabular}

Weights for fair animals:

Animal ID

Weight on last visit
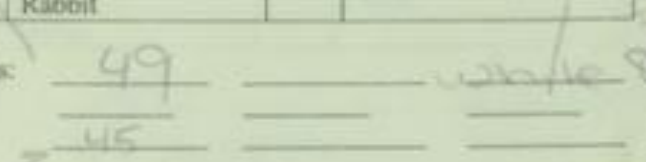

Number of Days since last visit:

Rute of Guin:

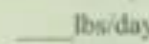

Ibsiday

lbsiday

Tbsiday

Number of dayor to fair.

Projected Weight Gain

Projected Wt (a) Fair

General Project Comments:
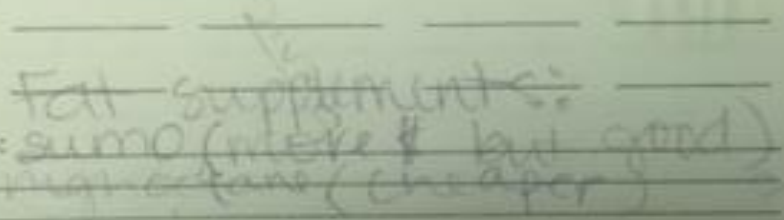

\section{Recommendations:}

Fair requirements to still meet: Signed Acknowledgemient Sheet Parent Exhibitor Meeting Entries turned into livestock office Other:

Student Signature
Nexd Project Vaisit

Date: LoV The Time:

Mon Tues Wed Thurs (Fri

Teacher Signature 
Supporting Completion

Materials F: Wall Chart of SAE Visits

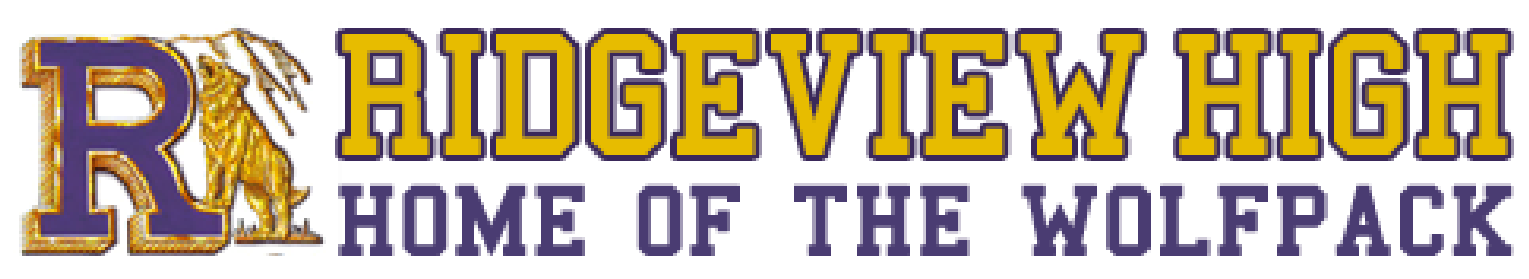




\section{F. Wall Chart of SAE Visits}

Student SAE Visits are assigned by me. I have created a google calendar that is linked to my website so students can access it and expect a visit. Visit locations vary, for example, the small stock owners bring their animals in to the classroom, and the large livestock are visited at the location of their animal. The calendar attached is my fair and summer calendar.

Supporting Completion Material Attached 
X potterton | Fair

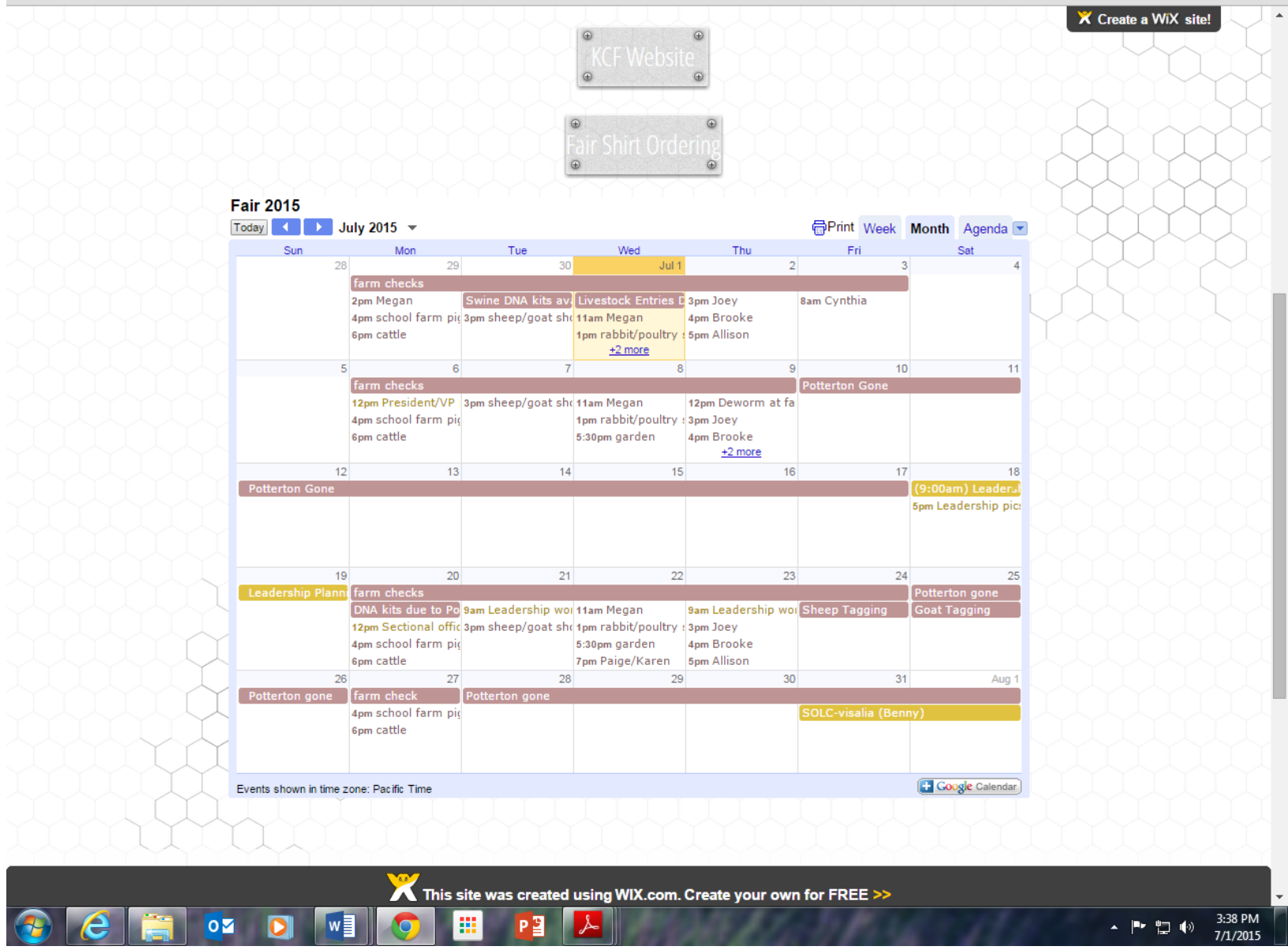


Supporting Completion

Materials G: SAE School

Board Approved Policy

Statement

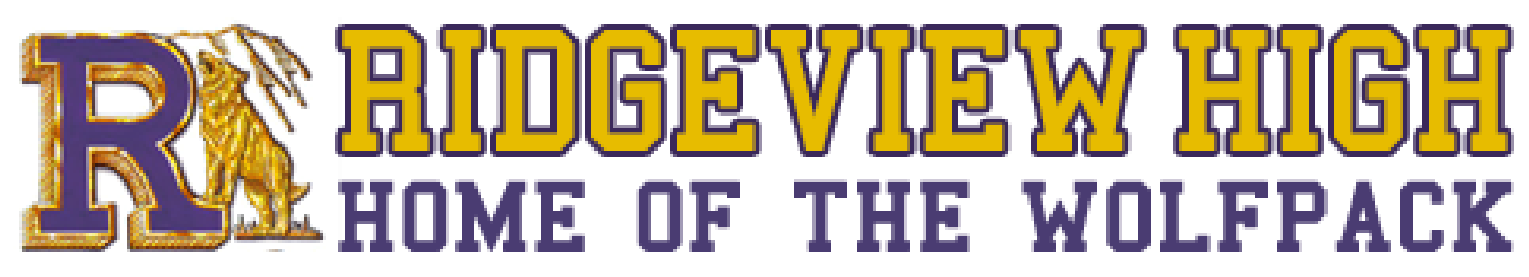




\section{G. SAE School Board Approved Policy Statement}

ACTIVITY/ATHLETIC CODE OF ETHICS

Preamble And Statement of Philosophy

The Activity and Athletic program in the Kern High School District shall be a part of the school curriculum. The programs shall be conducted in a way which provides an opportunity for worthwhile competition for all students.

It is the high school district's desire to see that proper ideals of sportsmanship, ethical conduct, and fair play, are realized by all school personnel, students, and spectators. Everyone concerned should realize that the purpose of a strong co-curricular program is to promote the physical, mental, moral, social, and emotional well being of the individual participant and other members of the student body. It must be remembered that, for all concerned, any organized event is only a contest and not a matter of life and death.

\section{CO-CURRICULAR ACTIVITIES}

Co-curricular activities, popularly known as extra-curricular activities, are privileges made available to students who agree to follow certain prerequisites and standards of conduct. These standards are in addition to the regular discipline rules for students attending regular classes.

It is important to note that the law grants authority to school officials to supervise students when they are participating in cocurricular activities or other supervised school-sponsored events held away from the school grounds. The Board of Trustees specifically notes that where students act or behave to diminish the efficiency of the school system of the State and exert a harmful influence upon the younger pupils of the school, they are subject to being disciplined and/or subject to loss of privileges of participation in co-curricular activities.

The District Superintendent and his staff shall develop and publish two lists of district-approved cocurricular activities annually, which are subject to higher standards, which are imposed by this code.

A. One list will include those activities in which participants are under the jurisdiction of all prerequisites, rules, and regulations at any time and at any place during the school year or calendar year, as outlined in the athletic section of this code.

The enforcement of regulations shall be the same as those listed in the athletic section of this code.

B. The second list will include those activities in which participants only come within these prerequisites, rules, and regulations under the following conditions:

1. The students are actually participating in, rehearsing for, or traveling to and from such activity while under the jurisdiction of school authorities; and

2. Such participation, etc., is outside of the regular classroom environment and/or regular instructional program.

The regulations which shall prevail for activities on this list shall be the same as those listed under "KHSD Regulations Governing Violations/Offenses by Students On and Off Campus" except that school jurisdiction is limited to the conditions listed under 1 and 2 above. 
The enforcement of regulations shall be the same as those listed in the athletic section of this code except that the forfeiture of privileges apply only to eligibility for performances, appearances, special rehearsals, competitions, etc., and not to the removal from the classroom instructional program.

\section{AGREEMENT TO FOLLOW ALL PREREQUISITES, RULES, AND REGULATIONS}

The agreement to follow all prerequisites, rules, and regulations will be the same as for athletics except that all references to "athletics" shall read "activities."

\section{SUMMARY}

In addition to regular discipline rules, the athletic and cocurricular activities codes apply additional standards to all students involved in certain school affairs, on or off campus.

(b) Summer school courses that are not repeat classes from the prior semester may be averaged with the second semester courses taken and a student may gain academic eligibility.

(c) If a student fails a class in the fall semester, he/she may re-take the class in summer school and use the grade/credits to gain athletic eligibility.

4. Grades earned in courses taken through Bakersfield Adult School will be used to determine a student's eligibility in the same manner as any other course taught in the KHSD. It is the responsibility of the high school to verify quarter grades for students enrolled in evening classes.

617.7.3 Eligibility for a freshman student will be determined at the end of the student's first grading period in high school.

617.8 Activities Exempt from Eligibility Standards A co-curricular program that has as its primary goal the improvement of academic or educational achievements of students is not subject to these eligibility requirements.

617.8.1 Programs in choral music, instrumental music, drama, forensics, journalism, dance, art, and agriculture are not subject to these eligibility requirements, except as noted in

617.8.2.

1. Any additional activities exempted from eligibility standards shall be determined by the Associate Superintendent for Instruction.

2. All such exempt activities must be a graded part of a class which receives course credit and which includes preparation for public performance as a major portion of course content.

617.8.2 A student enrolled in programs indicated in 617.8.1 who does not meet the extra-co-curricular academic eligibility requirements in 617.7 .2 shall not be allowed to participate in any part of that program which causes him/her to miss any class time during the regular instructional day.

1. Exceptions to this provision include:

(a) Future Farmers of America showing projects at fair only on show day.

(b) Fine Arts rating activities sponsored by the Kern High School District or other agency within Kern County.

2. Questions relating to exception (s) should be referred to the Associate Superintendent of Instruction.

617.8.3 Arrangements for assisting ineligible students to regain their eligibility status should be developed at the local school and should be publicized to students and parents. 617.8.4 A student's grade in a class shall not be adversely affected if that student is denied the right to attend field trips on co-curricular activities due to a G.P.A. of less than 2.0 . 
Supporting Completion Materials H: Program of Work

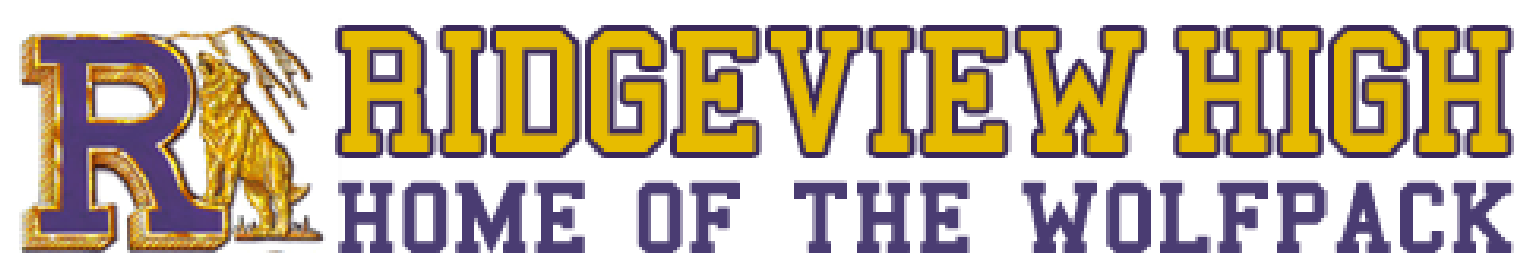




\section{H. Program of Work}

At this time, our chapter does not have a current POW. As my master's project, I have developed a POW for the current school. I hope that in the future, the officer team will be able to update and make adjustments to the POW now that there is a starting point. 
Supporting Completion

Materials I: FFA School

Board Approved Policy

Statement

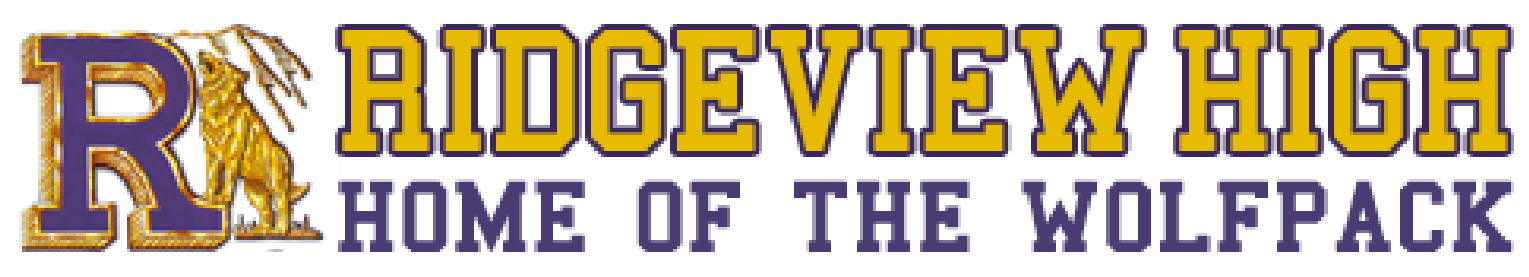




\section{FFA School Board Approved Policy Statement}

\section{ACTIVITY/ATHLETIC CODE OF ETHICS}

Preamble And Statement of Philosophy

The Activity and Athletic program in the Kern High School District shall be a part of the school curriculum. The programs shall be conducted in a way which provides an opportunity for worthwhile competition for all students.

It is the high school district's desire to see that proper ideals of sportsmanship, ethical conduct, and fair play, are realized by all school personnel, students, and spectators. Everyone concerned should realize that the purpose of a strong co-curricular program is to promote the physical, mental, moral, social, and emotional well being of the individual participant and other members of the student body. It must be remembered that, for all concerned, any organized event is only a contest and not a matter of life and death.

\section{CO-CURRICULAR ACTIVITIES}

Co-curricular activities, popularly known as extra-curricular activities, are privileges made available to students who agree to follow certain prerequisites and standards of conduct. These standards are in addition to the regular discipline rules for students attending regular classes.

It is important to note that the law grants authority to school officials to supervise students when they are participating in cocurricular activities or other supervised school-sponsored events held away from the school grounds. The Board of Trustees specifically notes that where students act or behave to diminish the efficiency of the school system of the State and exert a harmful influence upon the younger pupils of the school, they are subject to being disciplined and/or subject to loss of privileges of participation in co-curricular activities.

The District Superintendent and his staff shall develop and publish two lists of district-approved cocurricular activities annually, which are subject to higher standards, which are imposed by this code.

A. One list will include those activities in which participants are under the jurisdiction of all prerequisites, rules, and regulations at any time and at any place during the school year or calendar year, as outlined in the athletic section of this code.

The enforcement of regulations shall be the same as those listed in the athletic section of this code.

B. The second list will include those activities in which participants only come within these prerequisites, rules, and regulations under the following conditions:

1. The students are actually participating in, rehearsing for, or traveling to and from such activity while under the jurisdiction of school authorities; and

2. Such participation, etc., is outside of the regular classroom environment and/or regular instructional program.

The regulations which shall prevail for activities on this list shall be the same as those listed under "KHSD Regulations Governing Violations/Offenses by Students On and Off Campus" except that school jurisdiction is limited to the conditions listed under 1 and 2 above. 
The enforcement of regulations shall be the same as those listed in the athletic section of this code except that the forfeiture of privileges apply only to eligibility for performances, appearances, special rehearsals, competitions, etc., and not to the removal from the classroom instructional program.

\section{AGREEMENT TO FOLLOW ALL PREREQUISITES, RULES, AND REGULATIONS}

The agreement to follow all prerequisites, rules, and regulations will be the same as for athletics except that all references to "athletics" shall read "activities."

\section{SUMMARY}

In addition to regular discipline rules, the athletic and cocurricular activities codes apply additional standards to all students involved in certain school affairs, on or off campus.

(b) Summer school courses that are not repeat classes from the prior semester may be averaged with the second semester courses taken and a student may gain academic eligibility.

(c) If a student fails a class in the fall semester, he/she may re-take the class in summer school and use the grade/credits to gain athletic eligibility.

4. Grades earned in courses taken through Bakersfield Adult School will be used to determine a student's eligibility in the same manner as any other course taught in the KHSD. It is the responsibility of the high school to verify quarter grades for students enrolled in evening classes.

617.7.3 Eligibility for a freshman student will be determined at the end of the student's first grading period in high school.

617.8 Activities Exempt from Eligibility Standards A co-curricular program that has as its primary goal the improvement of academic or educational achievements of students is not subject to these eligibility requirements.

617.8.1 Programs in choral music, instrumental music, drama, forensics, journalism, dance, art, and agriculture are not subject to these eligibility requirements, except as noted in

617.8.2.

1. Any additional activities exempted from eligibility standards shall be determined by the Associate Superintendent for Instruction.

2. All such exempt activities must be a graded part of a class which receives course credit and which includes preparation for public performance as a major portion of course content.

617.8.2 A student enrolled in programs indicated in 617.8.1 who does not meet the extra-co-curricular academic eligibility requirements in 617.7 .2 shall not be allowed to participate in any part of that program which causes him/her to miss any class time during the regular instructional day.

1. Exceptions to this provision include:

(a) Future Farmers of America showing projects at fair only on show day.

(b) Fine Arts rating activities sponsored by the Kern High School District or other agency within Kern County.

2. Questions relating to exception (s) should be referred to the Associate Superintendent of instruction.

617.8.3 Arrangements for assisting ineligible students to regain their eligibility status should be developed at the local school and should be publicized to students and parents.

617.8.4 A student's grade in a class shall not be adversely affected if that student is denied the right to attend field trips on co-curricular activities due to a G.P.A. of less than 2.0 . 
Supporting Completion

Materials J: Agriculture

Department Recruitment

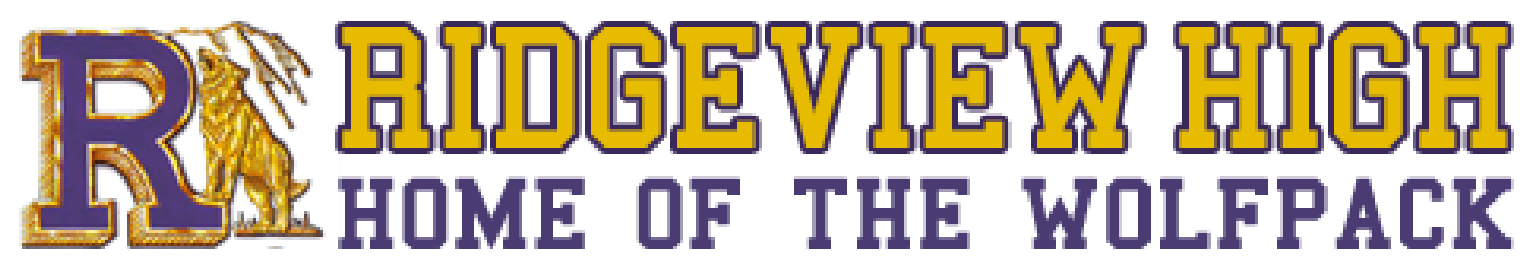




\section{J. Agriculture Department Recruitment}

The primary recruitment that occurs for the program is during Freshman Orientation. At this, I briefly speak to the incoming students and their parents. We also set up a booth where the current students are able to talk to potential students. At the booth we had a board of pictures, flowers from our garden, awards, brochures, and a card to contact me about fair information. Students also walked around with the brochure. I created the brochure this year and made sure to focus on including information about credits earned and how ag fits with the rest of their courses.

Within the school, we hosted daily lunch activities, dress up days, and a staff and member breakfast during FFA week. Also, students are included in the daily announcements to celebrate successes within our chapter.

Supporting Completion Material Attached 
FFA Week

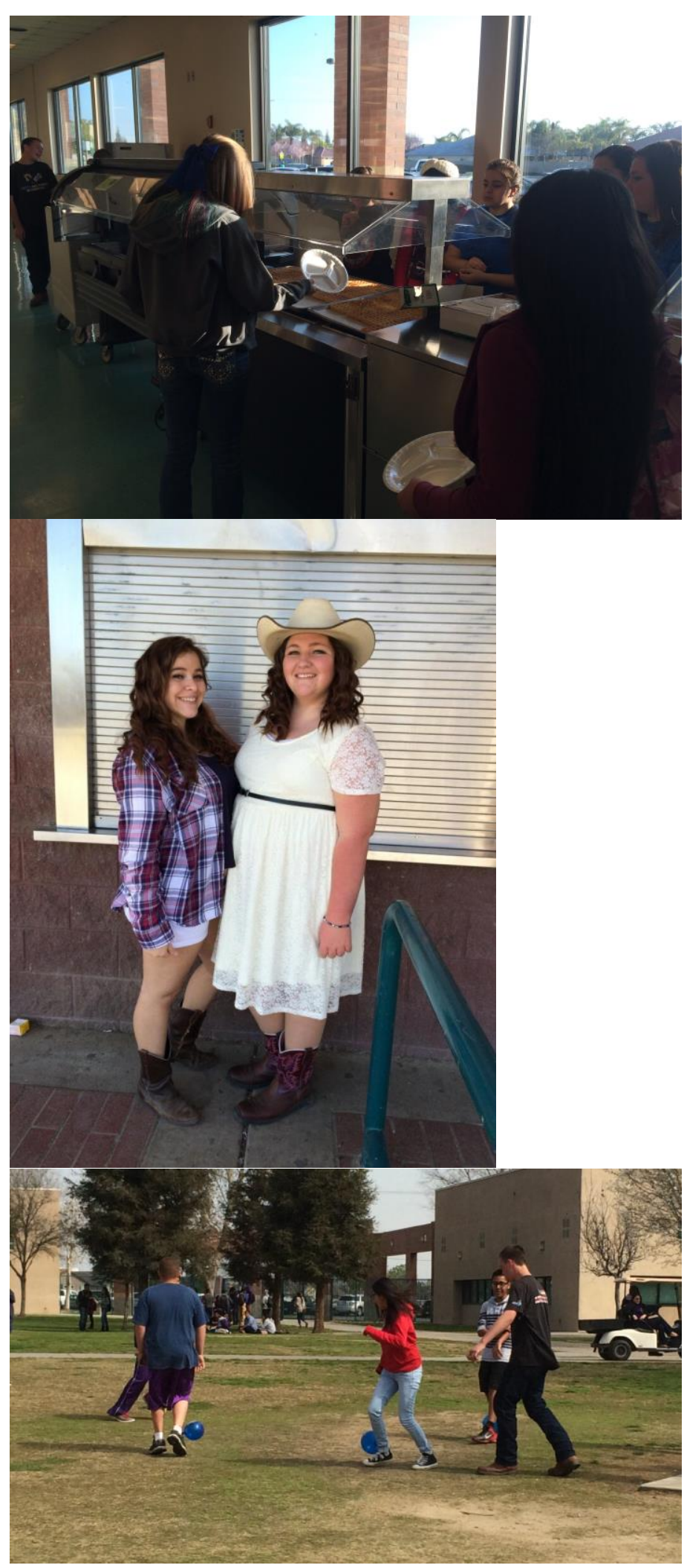


Freshman Orientation

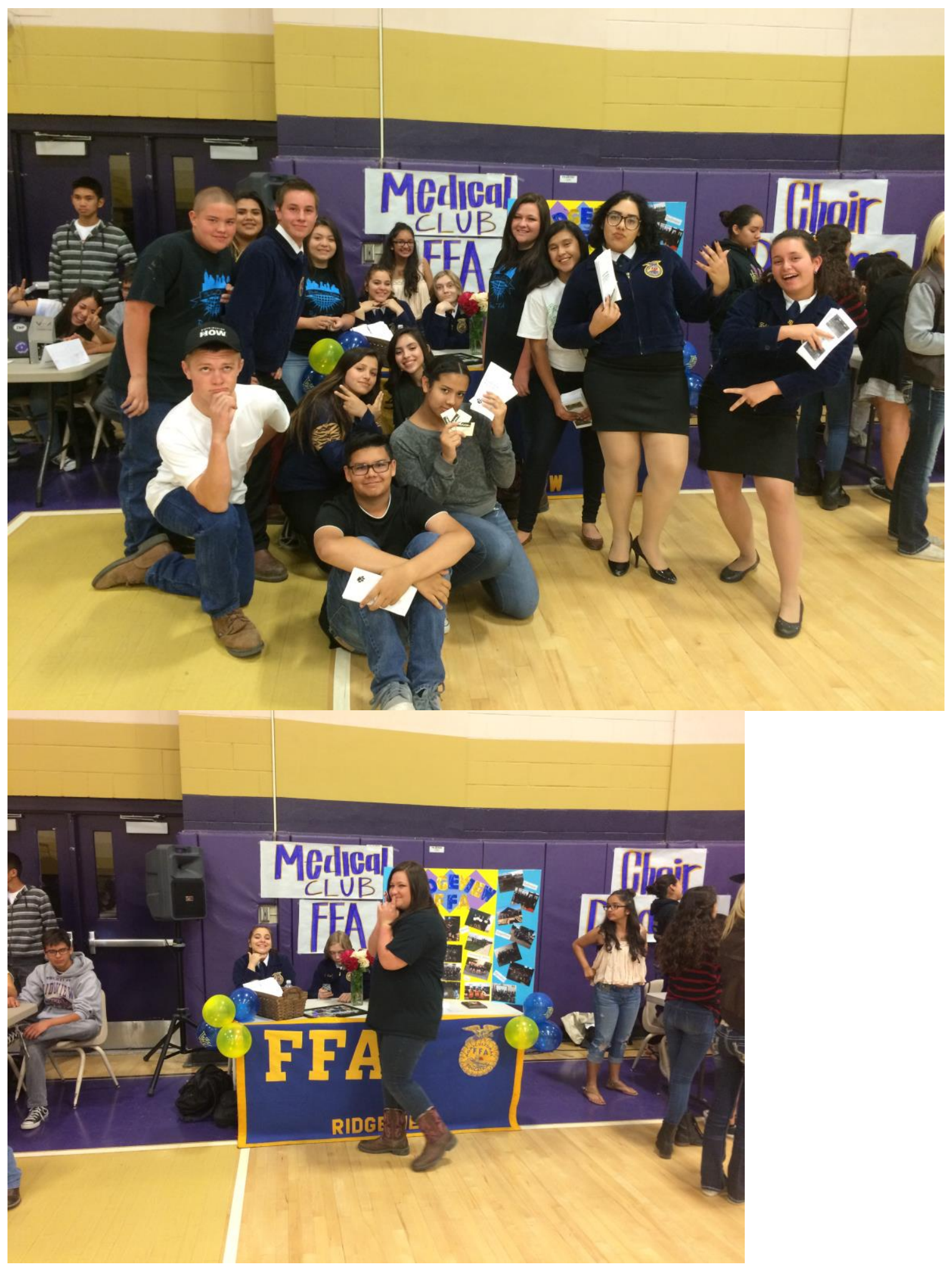




\section{Brochure}

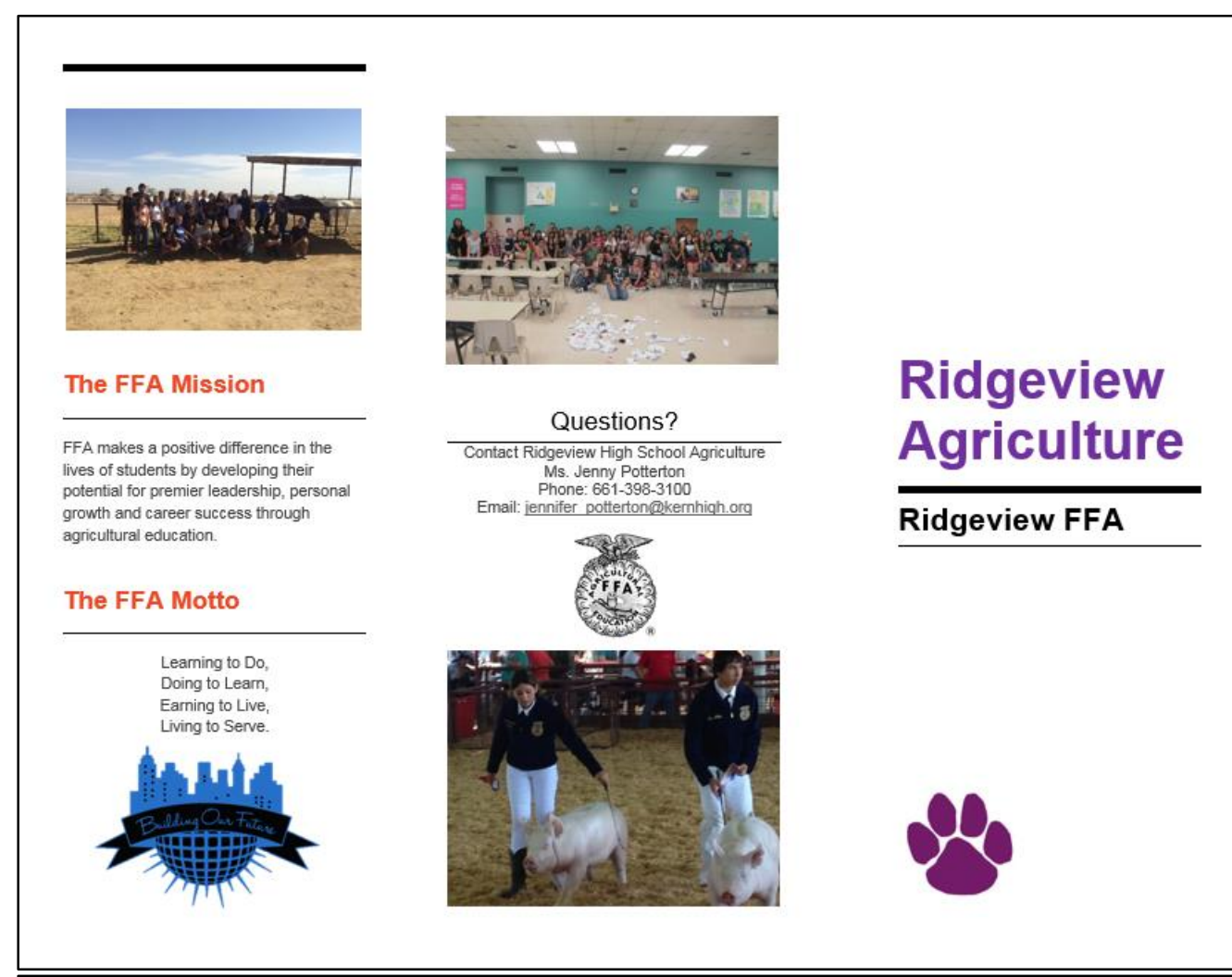

What Does Ag Education Offer Me?

- Agricultural education prepares students for successful careers and a lifetime of informed choices in the global agriculture, food, fiber and natural resources systems.

- Through agricultural education, students are provided opportunities for leadership development, personal growth and career success. Agricultural education instruction is delivered through three major components:

- Classroom/Laboratory instruction
(contextual learning)

- Supervised Agricutural Experience programs (work-based leaming) Student leadership organization (National FFA Organization).
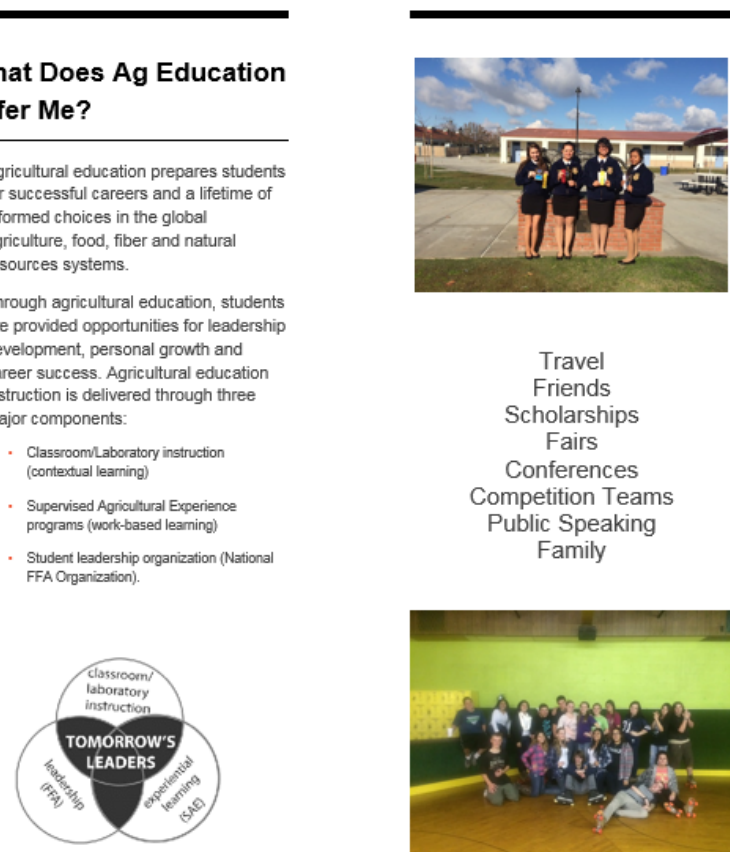

How Does Agriculture Fit In Your Schedule?

$9^{\text {th }}$ Grade

-Ag Earth Sciencers

English 9

-Algebra/Geometry

-Spanish

-Health

$10^{\text {th }}$ Grade

$-\mathrm{Ag}$ Biology

Friends

Scholarships

Fairs

Conferences

Competition Teams

Public Speaking

Family

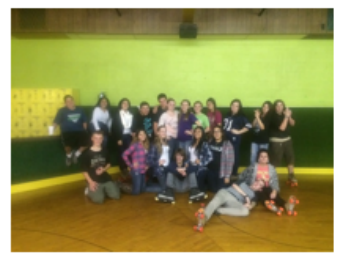

-Spanish 2

-Visual or Performing Arts

$11^{\text {th }}$ Grade

-Vet Science ${ }^{\star / A g}$ Sales

-Vet Science*//Ag Sales
-English 11
-Adv. Algebra/Math Anslysis

-Spanish 3

-US History

$12^{\text {th }}$ Grade

-Vet Science
- English 12

-Geometry/Adv. Algebra

Spanish AP

- Government'Economics
Anatomy/Geology/AP BiologyiPhysics

-UC Lab Scienoe Credit ("व") 
Supporting Completion Materials K: FFA Chapter Scrapbook

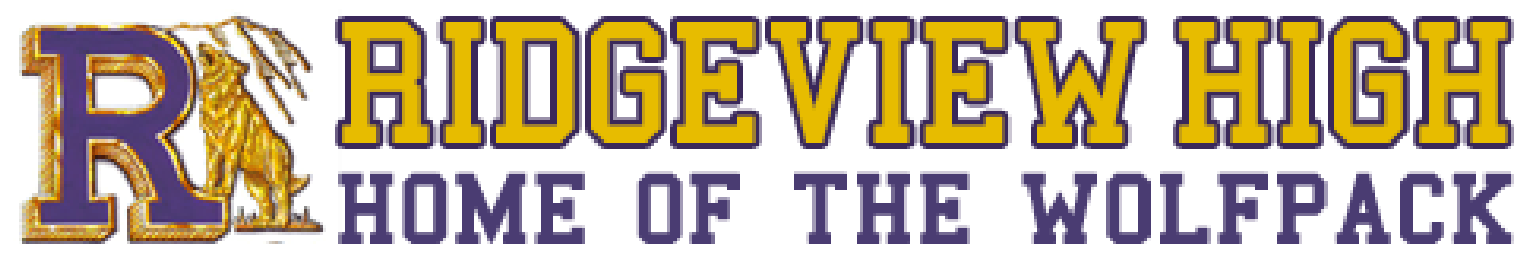




\section{K. FFA Chapter Scrapbook}

The Ridgeview FFA Chapter doesn't have an up to date scrapbook that is maintained on a regular basis. This is an area where improvement could happen, but at this point in time, the decision has been made that money could be saved on supplies for the scrapbook and into other areas of the FFA. However, there are records of the successes and activities of the Ridgeview FFA Chapter kept on file in the agriculture department. Additionally, there are banquet slide shows presented bi-annually and a collage of pictures posted in the room at the beginning of the year as a year in review. I have found that student gravitate towards this more than the scrapbook.

Supporting Completion Material Attached 
Winter Banquet Slide Show
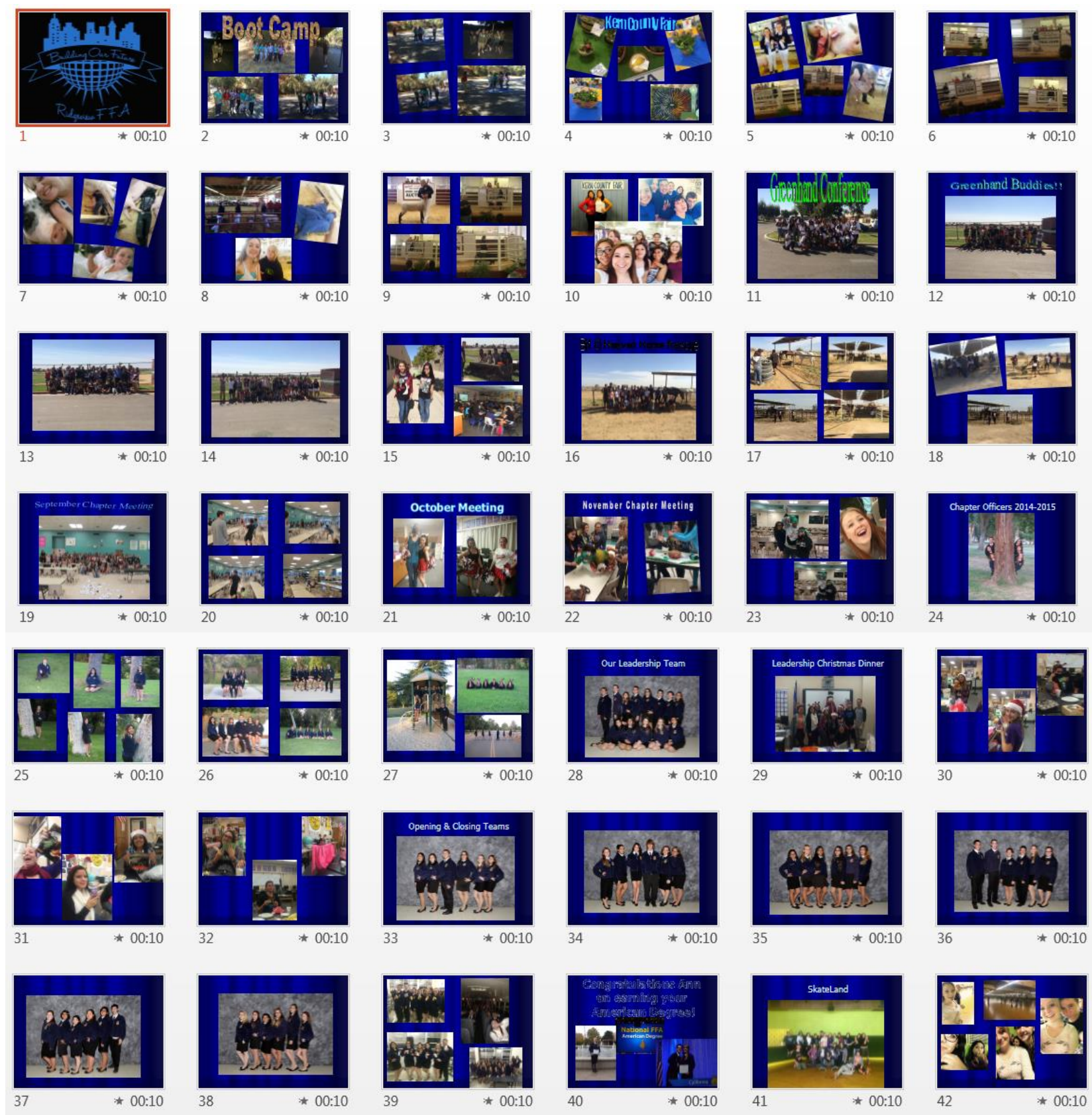
Spring Banquet Slide Show
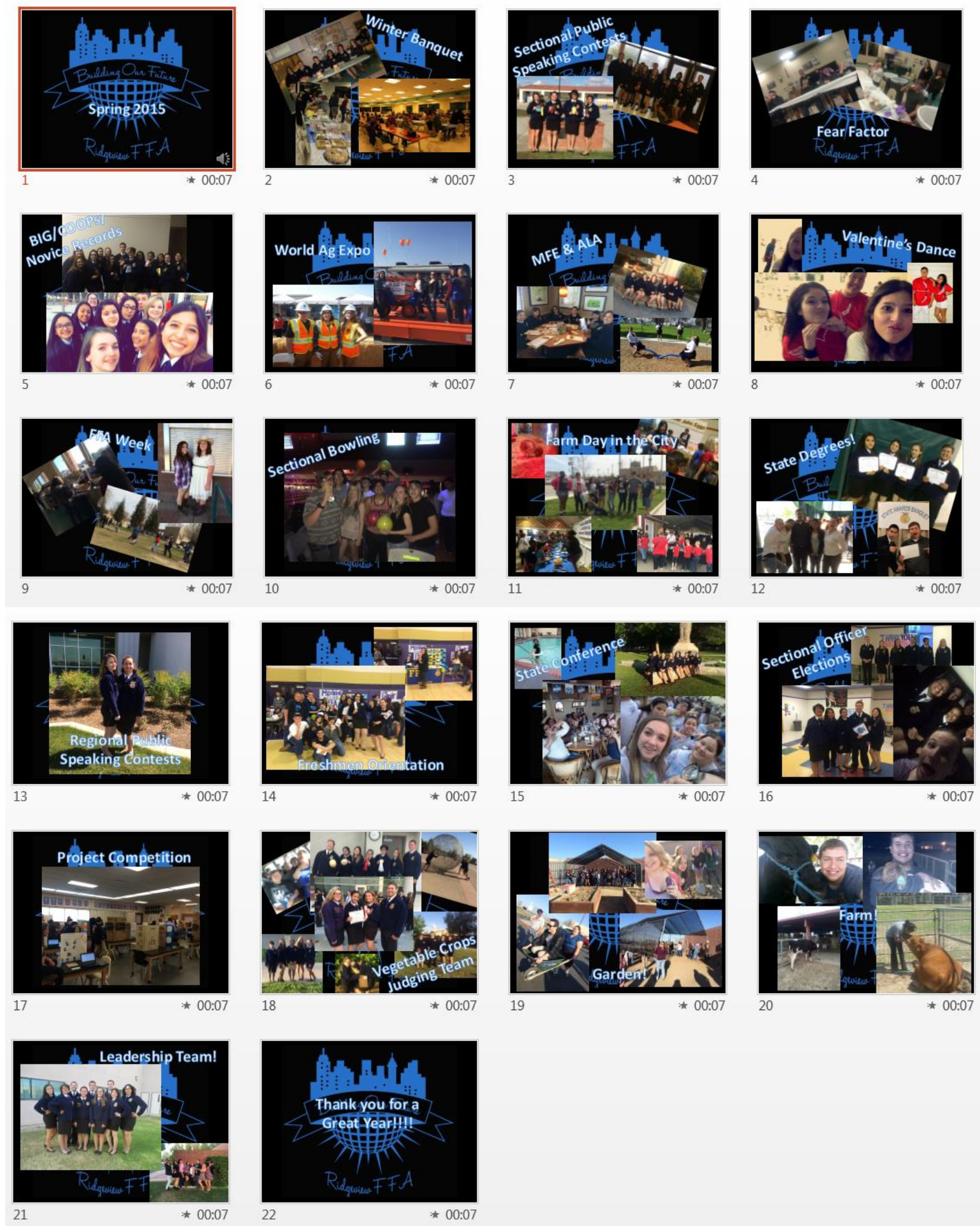
End of the Year Collage

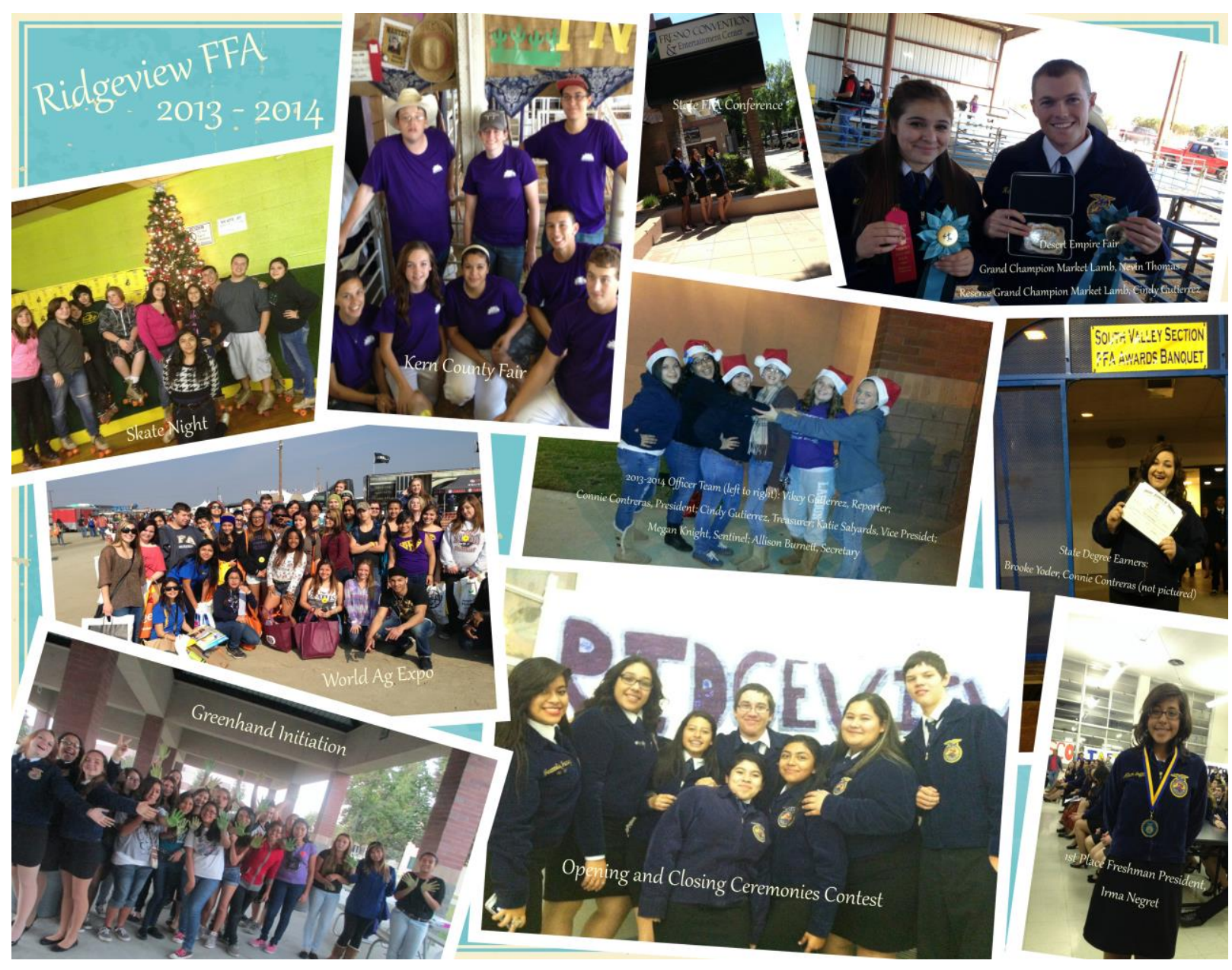


Supporting Completion

Materials L: Summer

Activities Calendar

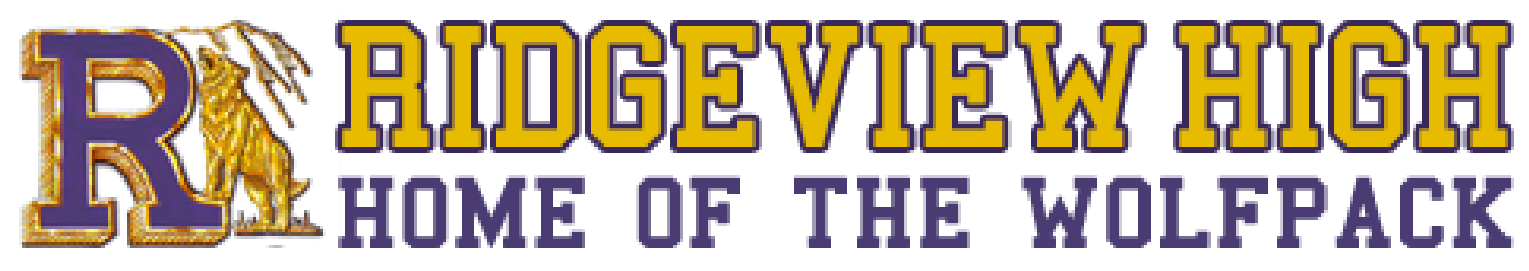




\section{Summer Activities Calendar}

My summer activities calendar is posted on my website and sent to the district. The one on my website is updated as the summer goes because of schedule changes that may occur.

Supporting Completion Material Attached 

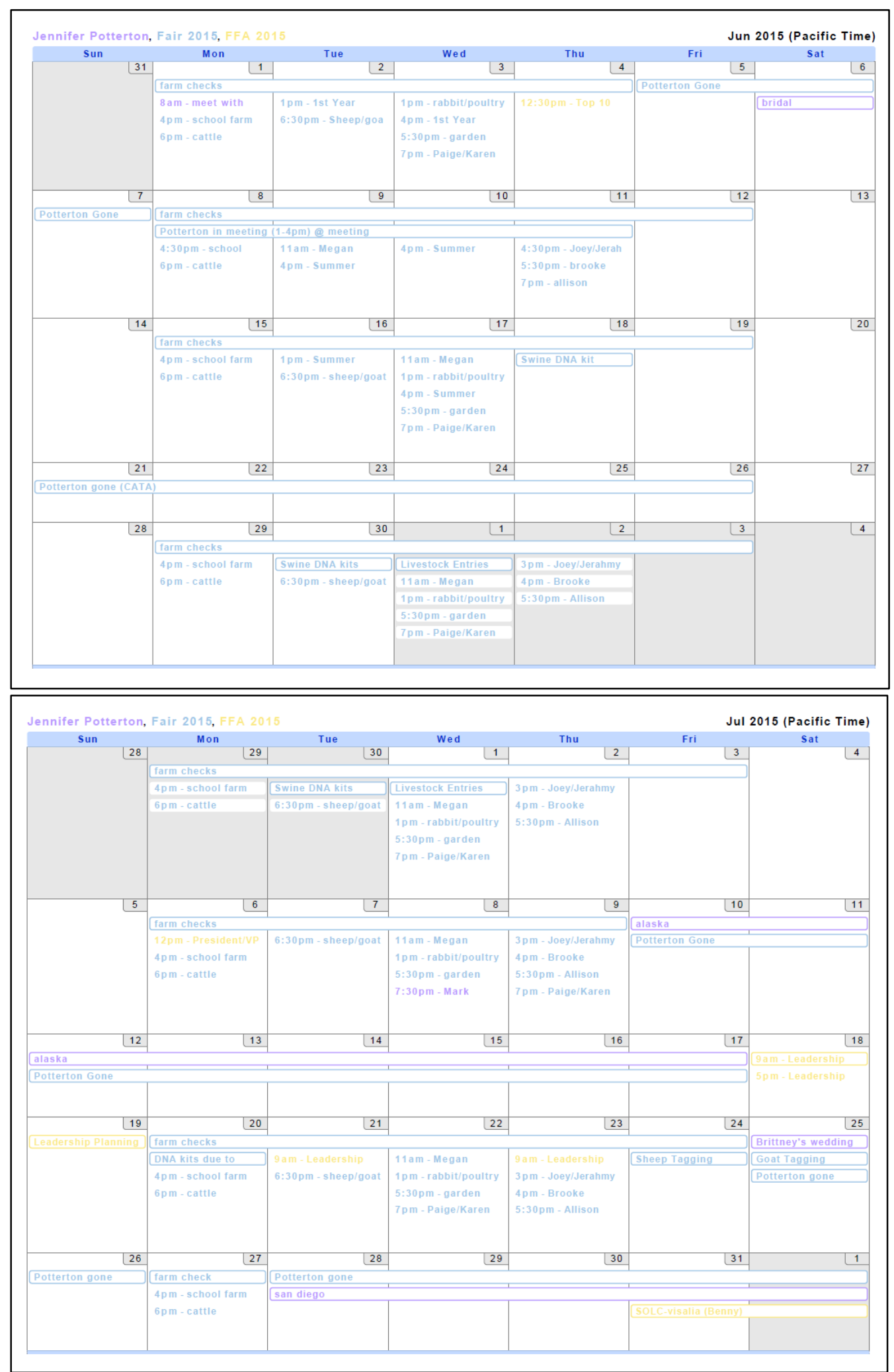


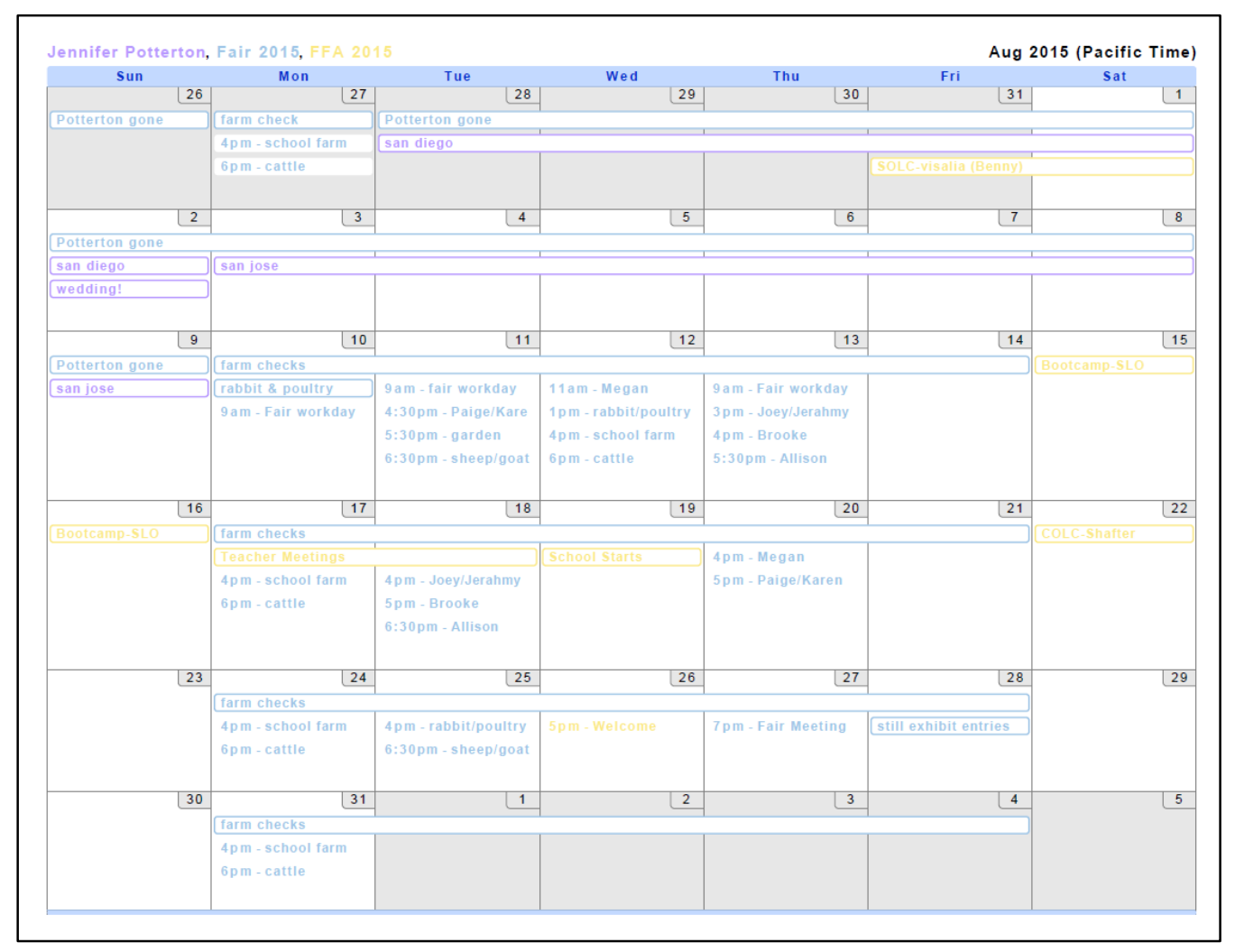


Supporting Completion

Materials M: Graduate

Follow-Up Survey

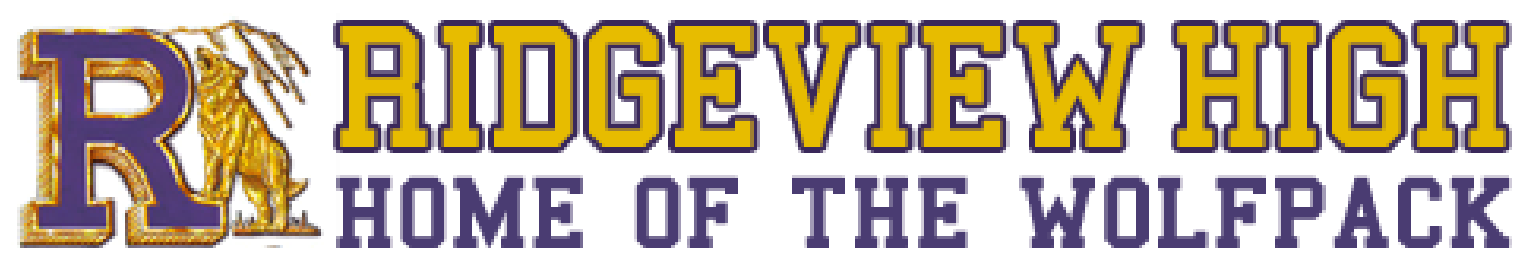




\section{Graduate Follow-Up Survey}

At the end of the school year when I was using the student data sheets to instruct the students on registering for their next ag class, I had the students update the information on their Student Data Sheets to reflect their post-graduation plans. This was my first time using this method, so it is a test run this time. In the future, I may create a worksheet or Google Form for them to complete for the information.

Supporting Completion Material Attached 


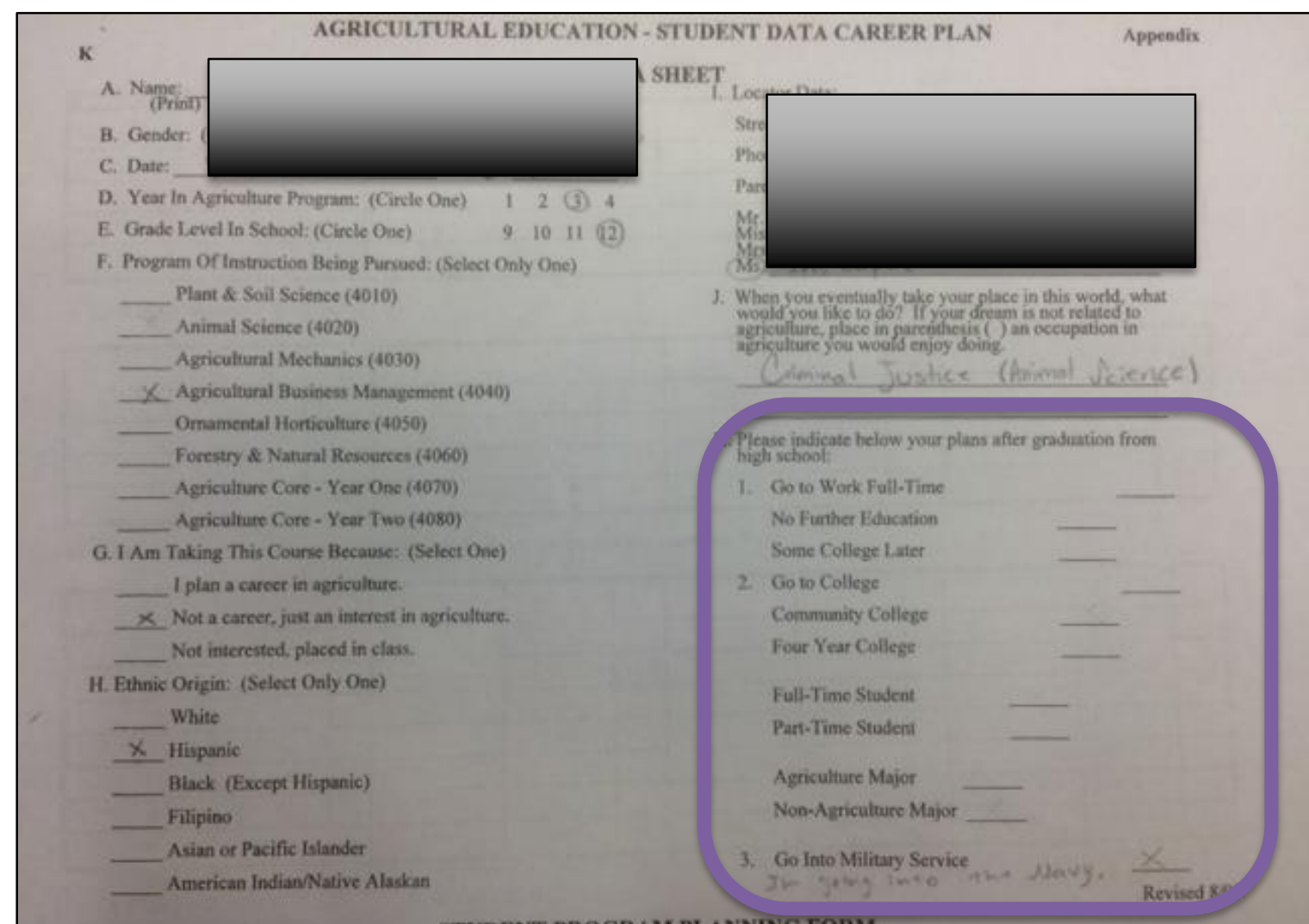

STUDENT PROGRAM PL.ANNING FORM

L. Planned coune of study to mect occupational goal. By school year, list all claseses peeviously taken, currently being taken, and planned to be taken in

K

A. Nanne:

B. Giende

C. Date

D. Year in Agricultare Program: (Circle One) (T) 234

E. Grade Level in School: (Circle Onc) $\quad(9)(10) / 11$ i2

F. Program Or Instruction Being Prarsised: (Select Only One) Plant \& Soil Science (4010) Animal Science (4020)

Agricultural Mechanics (4039)

___ Agricultural Business Management (4040) Omamental Honticuliure (4050)

Forretry \& Natural Resources (4060)

7 Apriculture Caro - Year One (4070) Agriculture Cote - Year Two (4080)

G. I Am Taking This Coune Because: (Seloct One)

I glan a career in apriculture

X. Not a carcer, just an interest in agriculture. Not interested, placod in class.

H. Ethnic Origin: (Select Only One)

White
Hispanic
Hlack IEx

Black (Except Hispatic)

Filipino

__ Asian or Pacific Blandet

Americas Indian/Native Alaskan
Pease indicate below your plans after graduation froes

Co to Wark Full-Time

No Funthet Bdacation

Full-Time Stadent

Agriculture Major

Go Into Military Service 
A. Name:

B. Gender

C. Daie:

D. Year In

E. Grade Level In Sehool-(Cincle One)

Frogram Or Instruction Being Pursued: (Select Only One) Plant \& Soil Seience (4010) Animal Science (4020)

Agricultural Mechanics (4030)

$\checkmark$ Agricultural Business Management (4040)

Omamental Horticulture (4050)

Forestry \& Natural Resources (4060)

Agriculture Core - Year One (4070)

Agriculture Core - Year Two (4080)

G. I Am Taking This Course Because: (Select One)

I plan a career is apriculture.

$\checkmark$ Not a career, just an intereat in agriculture.

H. Ethnic Origin: (Select Oaly One)

H. Ethnic Origin: (Select Oaly One)

Whise
$\checkmark$ Hispanic
Black (Execpt Hispanic)
Filipino
Asian or Pacifie Islander
American Indian/Native Alaskan

SHEET

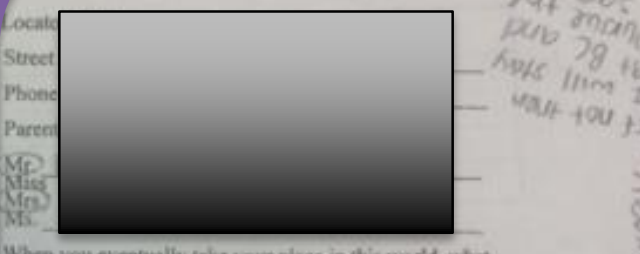

When you cventually gake your place in this worlid, what

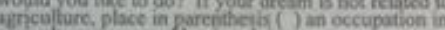

finculture you would enjoy dome

I. unin wie in be placed

in this worid as a

Pease indicate below your plass ather maduation from

1. Ge to Woek Full-Time No Further Edocation Some College L Later

2. Co to College Community College Four Year College Full-Time Student Pan-Tiues Student Agricultare Major Non-Agriculture Major

3. Go Into Military Service

\section{as}

STUDENT PROGRAM PLANA VG FORM 
Supporting Completion

Materials N: Graduate

Status

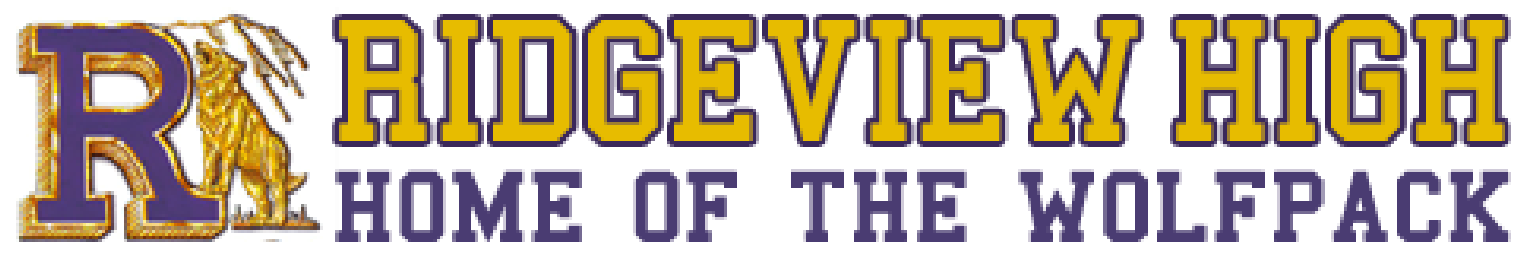




\title{
N. Graduate Status
}

\author{
Graduate Follow-up
}

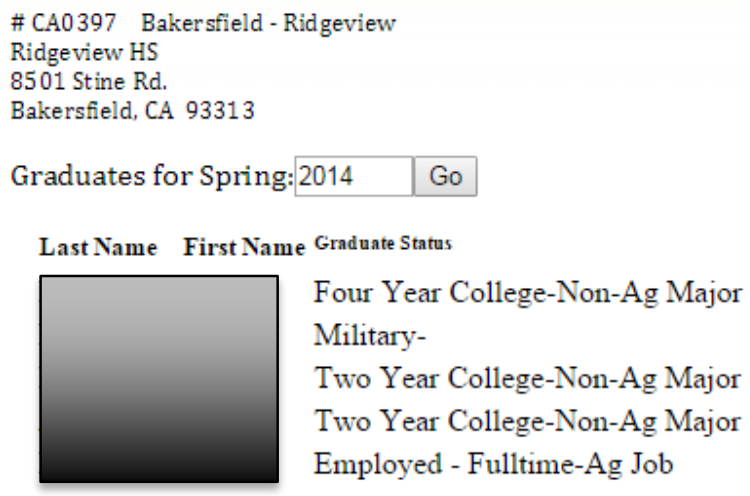

Printed: 7/2/2015 11:31:48 AM

Count: 5 
Supporting Completion

Materials 0: Vo-Ag

Comprehensive Plan

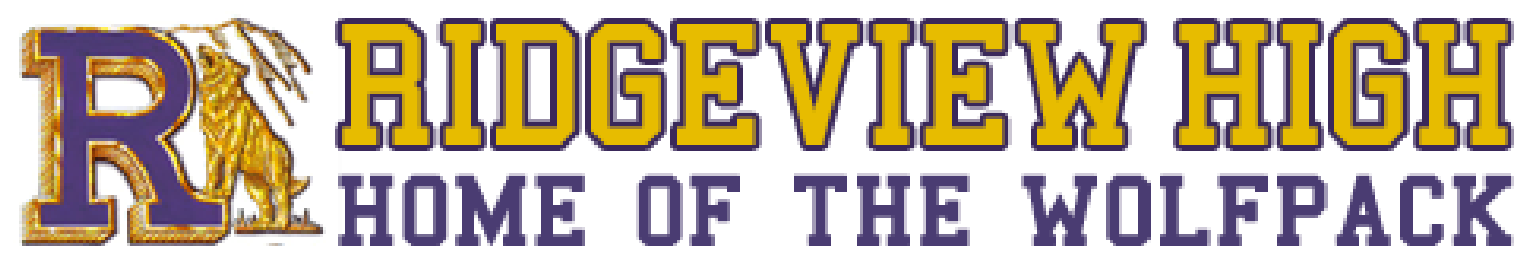




\section{Vo-Ag Comprehensive Plan}

The Program Plan is a mixture of out of date materials and updated information. I have slowly been working to update information as I go. Annually information related to the plan is submitted to Mr. Parker.

Supporting Completion Material Attached 


\section{Program Plan Part A}

\section{Job Market Description}

Ridgeview High School is located approximately 5 miles south west of the city of Bakersfield. Our occupational area would consist of Kern County. The major production in our occupational area consists largely of: cotton, grapes, cattle and calves, tomatoes, milk, alfalfa hay, oranges, plums, nectarines, seed cotton, turkeys, seed alfalfa, carrots, dairy, wheat, and peaches. This occupational area is also extremely rich in agriculture mechanics, ornamental horticulture, agricultural supplies and service, agricultural products and processing, agricultural resources and recreation, and forestry.

California is a major producer of agricultural products and is recognized as the leading state in the production of many products. The top ten California commodities are milk and cream, grapes, cattle and calves, nursery products, cotton lint, flowers and foliage, almonds, head lettuce, hay and strawberries. This is all done on 38 million acres.

The United States has land resources that for surpass that of many other countries. More than one billion acres of the United States land mass are established farms and ranches, with another seven hundred and fifty million acres covered with forest lands. The United States produces all of the 10 major crops grown in the world. Some include corn, soybeans, wheat, barley, cotton, potatoes, sorghum, sugar(cane \& beets), and rice. 


\section{Program Plan Part B}

\section{Targeted Occupations in Agriculture Production}

\section{Animal Science}

Animal Science in the San Joaquin Valley consists of beef, dairy, sheep, swine, poultry, goats and horses. Operations consist of breeding to feedlot and processing of animals and products. Some of the lambs are shipped north for feeding, but many are used to graze on alfalfa fields in the area. Cattle are often shipped to the foothills for winter grazing. Many of the locally produced animals are fed out here in the valley.

\section{Job Titles}

1.) Manager or Shepherd

3.) Veterinarian

5.) Cowboy Farmhand

7.) Truck Driver

9.) 4-H County Advisor

11.) Auctioneer

13.) Marketing Agent

15.) Animal Transporter

17.) Insurance Sales

19.) U.C. Extentionist
2.) Nutritionist

4.) Veterinarian Technician

6.) Equipment Technician

8.) Feed Mill Operator

10.) Custom Meat Cutter

12.) Sales Yard Manager

14.) Cattle Broker

16.) Meat Inspector

18.) Geneticist

\section{Plant Science}

Plant Science consists of the production of crops such as: cotton, grapes, alfalfa hay and seed, tomatoes, lettuce, melons, wheat, oranges, plums, peaches, nectarines, and a variety of other crops. The San Joaquin Valley offers many jobs in the field of plant science.

Job Titles

1.) Manager Supervisor

3.) Truck Driver

5.) Economist

7.) Entomologist

9.) USDA Inspector

11.) Truck Dispatcher

13.) Vegetable Broker

15.) Chemical Advisor

17.) Packing Shed Worker

19.) Weigh Master
2.) Owner Operator

4.) Custom Tractor Operator

6.) Chemical Applicator

8.) U.C. Research Specialist

10.) U.C. Extentionist

12.) Marketing Consultant

14.) Sales Representative

16.) Labor Contractor

18.) Irrigation Scheduler

20.) Plant Supervisor 


\title{
Program Plan Part C
}

\author{
Ridgeview High Agriculture
}

Department Goals

1.) Prepare young people for Agriculture Occupations.

2.) Advance agriculture education and give people an appreciation for agriculture.

3.) Train young people to become leaders in the community.

4.) Be a positive influence in a young person's life.

\section{Department Objectives}

1.) Each student will prepare a plan of career development with the program area of major agriculture interest.

2.) Each student will engage in Supervised Occupational Experience program by the end of the first year in agriculture.

3.) Each student shall participate in FFA activities and shall receive a grade for such.

4.) All graduates will be surveyed within a year after their graduation.

5.) Instruction offered by the Ridgeview Agriculture Department will reflect skills, knowledge and attitudes required for employment as determined from information gathered from graduate follow-up surveys and the Ag Advisory Committee.

6.) Staff members will continue to update their skills and competencies by attending professional development activities sponsored by the CATA and industry.

7.) All freshman students will receive a comprehensive course of study exposing them to the different aspects of agriculture education.

8.) The agriculture staff will assess the facilities and equipment to insure students of an education that is technologically intensive. 


\section{Program Plan Part D}

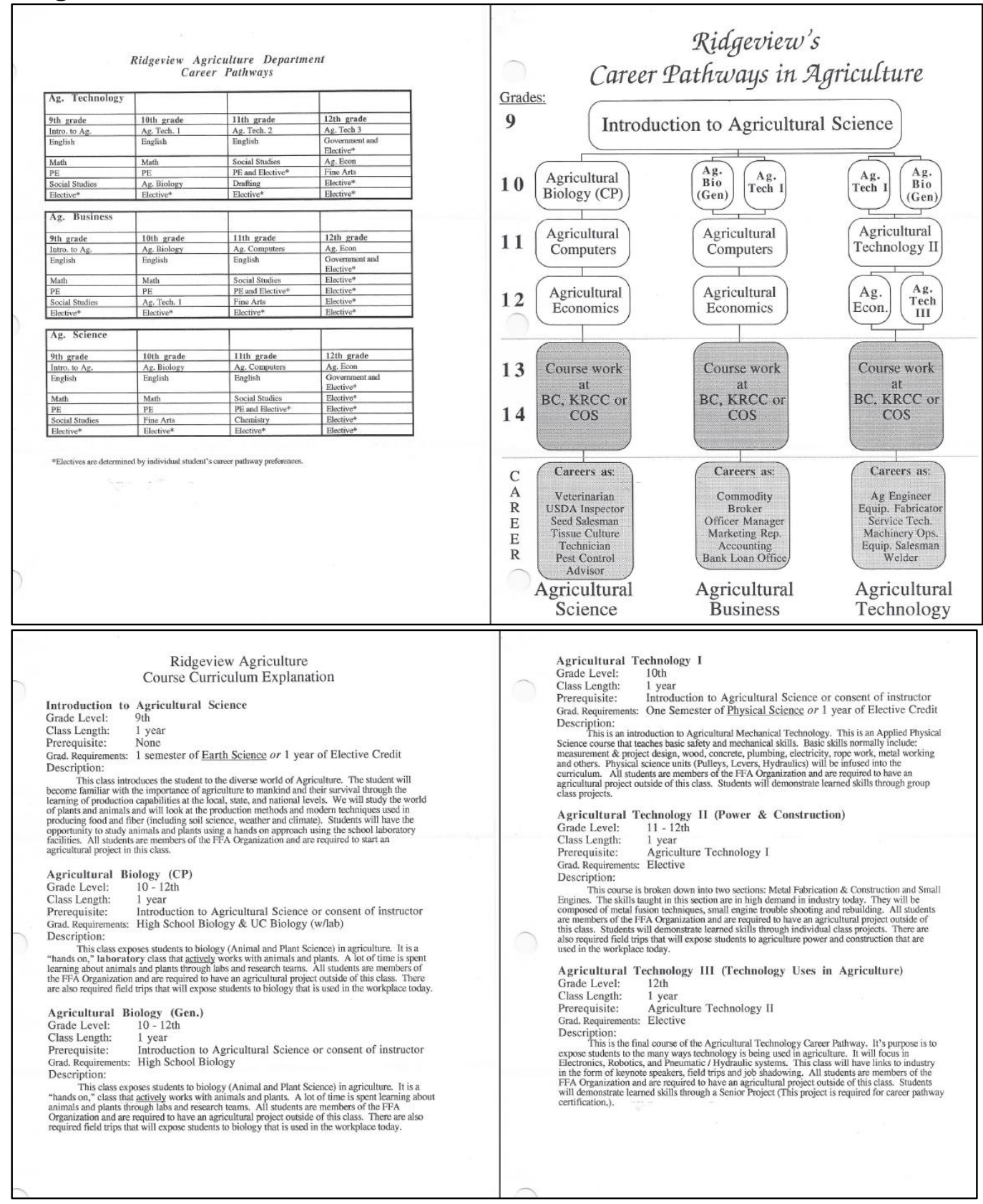




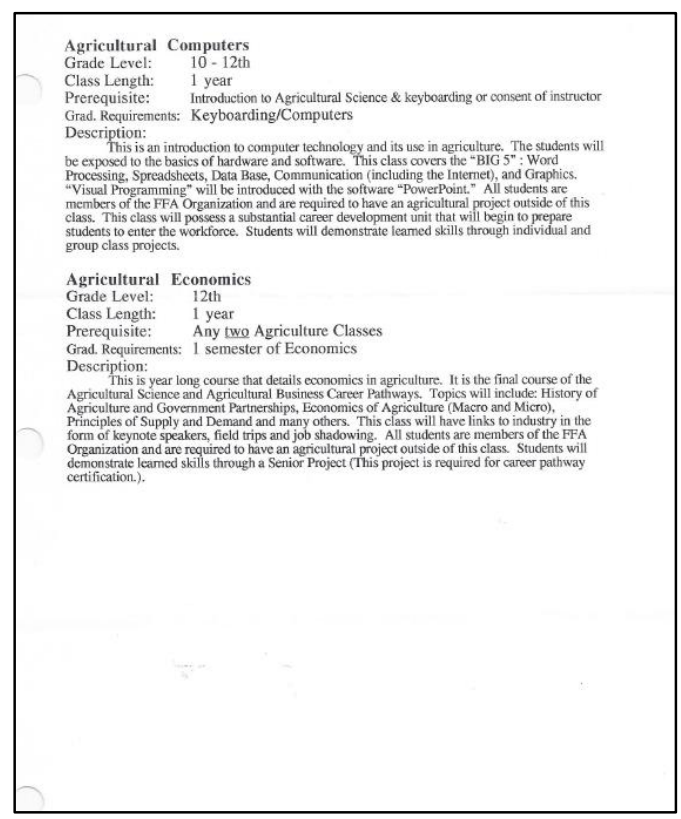




\section{Program Plan Part E}

Ag. Earth Science Course Outline

\begin{tabular}{|c|c|c|c|}
\hline Unit & Topic & Time Frame & Standards Addressed \\
\hline I. & California Agriculture & 2 woek & APS Cl.1.6 \\
\hline II. & Scientific Process & 2 weeks & $\begin{array}{l}\text { APS C13.1-3, AFS 1.2a-m, } \\
\text { I\&E 1, a-h }\end{array}$ \\
\hline III. & $\begin{array}{l}\text { Plate Tectonics: ocean floor } \\
\text { features, plate boundaries, rocks }\end{array}$ & 5 weeks & $\operatorname{AFS} 4,4, \operatorname{ES} 3 . a-c$ \\
\hline IV. & $\mathrm{FFA}$ & 3 week & $\begin{array}{l}\text { AFS } 1.1(10.0) \text {, AFS } 2.1-4 \text {, } \\
\text { AFS } 7.0-6, \text { AFS } 9.0-6 \text {, } \\
\text { AFS } 10.1\end{array}$ \\
\hline V. & $\begin{array}{l}\text { Plate Tectonics: earthquakes, } \\
\text { volcanoes }\end{array}$ & 2 weeks & ES $3, \mathrm{~d}_{4} \mathrm{e}$ \\
\hline V1. & Record Keeping & 2 weeks & $\begin{array}{l}\text { AFS } 1.1(10.0) \text {, APS C3.1- } \\
4, \text { AFS } 10.3\end{array}$ \\
\hline VII. & Califormia Gcology & 2 week & APS C10.1, ES 9, a-c \\
\hline VIII. & Agriculture Resources & 1 week & APS C2.1-5. ES 9.9. c \\
\hline IV. & Bio-Geochemical Cycles & 2 week & APS CI1.5-6, ES 7, a-c \\
\hline $\mathrm{X}$. & Earth's Atmosphere & 2 weeks & APS C10.3, ES 8. a-c \\
\hline $\mathbf{X I}$. & Energy and Agriculture & I week & APS E6.184, ES 4. b-c \\
\hline XII. & Heating the Earth's Surface & 2 weeks & ES 5. a-e \\
\hline XIII. & Earth's Climate & I week & $\begin{array}{l}\text { APS E2.1-6, APS F2,4, ES } \\
6 . \text { a-c }\end{array}$ \\
\hline XIV. & Astronomy & 2weeks & ES 1.a-f \& 2a-d \\
\hline $\mathrm{XV}$. & $\begin{array}{l}\text { Soils: formation, texture, } \\
\text { structure, erosion, and } \\
\text { conservation of }\end{array}$ & 4 weeks & APS E3.284 \\
\hline XVII & $\begin{array}{l}\text { Supervised Agriculture } \\
\text { Experience }\end{array}$ & 2 weeks & $\begin{array}{l}\text { APS C4.1-5, C6.1-2, C8.1- } \\
3, \text { C9.1-5, CII.1, AFS } 10.2\end{array}$ \\
\hline XVIII & $\begin{array}{l}\text { Agricultural Careers and } \\
\text { Development Events }\end{array}$ & 2 weeks & $\begin{array}{l}\text { AFS } 3.1-6, \text { AFS } 5.0-3 \\
\text { AFS } 11.0\end{array}$ \\
\hline
\end{tabular}

Ag. Biology Course Outline

\begin{tabular}{|c|c|c|c|}
\hline Unit/Quarter & Topic & $\begin{array}{c}\text { California } \\
\text { State Standards }\end{array}$ & $\begin{array}{c}\text { CTE/Agriculture } \\
\text { Standards }\end{array}$ \\
\hline I. Quarter 1 & Scientific Process & $\begin{array}{l}18 \mathrm{E} \text { c,d.f.j.l; Bio } \\
4 \mathrm{e}, 5 \mathrm{a}, 9 \mathrm{a}\end{array}$ & $\begin{array}{l}\text { 1.a,c,d,f,j,1,m } \\
\mathrm{C} 13,0 ; \mathrm{C} 13.1 ; \mathrm{C} 13.2 ; \mathrm{Cl} 3.3\end{array}$ \\
\hline II. Quarter 3 & Ecology & Bio $6 a-f$ & $\begin{array}{l}\mathrm{C} 2.1 ; \mathrm{C} 2.3 ; \mathrm{G} 1.0 ; \mathrm{G} 1.4 ; \mathrm{D} 7.0 ; \\
\mathrm{D} 7.2 ; \mathrm{E} 1.0\end{array}$ \\
\hline III. Quarter 1 & $\begin{array}{l}\text { Cellular Structure } \\
\text { and Process }\end{array}$ & Bio $1 c, e, f, g, h, l, j, 2 u$ & $\begin{array}{l}\mathrm{C} 5.0 ; \mathrm{C5} .1 ; \mathrm{C} 5.2 ; \mathrm{C} 5.3 ; \mathrm{C} 5.4 \\
\mathrm{C} 11.5 ; \mathrm{C} 11.4 ; \mathrm{C} 11.0\end{array}$ \\
\hline IV. Quarter 2 & Genetics & $\begin{array}{l}\text { Bio 1d, 2a-g, 3a-b, 4a-e, } \\
5 \mathrm{a}-\mathrm{c}, 7 \mathrm{~b}\end{array}$ & $\begin{array}{l}\mathrm{C} 7.0 ; \mathrm{D} 5.0 ; \mathrm{D} 5.4 ; \mathrm{D} 5.5 ; \\
\mathrm{C} 3.3 ; \mathrm{C} 3.4\end{array}$ \\
\hline V. Quarter 3 & Evolution & Bio 7a-d, 8a-e & $\mathrm{C} 4.1: \mathrm{C} 4.2$ \\
\hline X. Quarter 3-4 & $\begin{array}{l}\text { Human Anatomy } \\
\text { and Physiology }\end{array}$ & Bio 9b-e, 10a-e & $\mathrm{C} 6.0 ; \mathrm{C} 11.5 ;$ \\
\hline Quarter 4 & $\begin{array}{l}\text { Independent Lab } \\
\text { Based Projects, } \\
\text { CTE }\end{array}$ & $\begin{array}{l}\text { Bio 9a-l, } \\
\text { I\&Ea,b,c.d,f,g,k,1,m }\end{array}$ & $\begin{array}{l}2.0 \text { - Communication:2.1, } 2.2 \text {, } \\
2.3,2.7,2.8 \text {, Writing: } 1.3,1.5 \text {, } \\
2.3,2.5,2.6 \text {, Writing }\end{array}$ \\
\hline
\end{tabular}




\begin{tabular}{|l|l|l|}
\hline & & Strategies \& Applications: 1.6, \\
& & 2.6 \\
& Listening \& Speaking Skills: \\
& $1.1,1.7$, & $9.0-$ Lendership \& Team \\
& Work: $9.1,9.2,9.3$, \\
& $10.0-$ Technical Knowledge \& \\
& Skills: 10.2, 10.3, \\
\hline
\end{tabular}

Vet Science

\begin{tabular}{|c|c|c|c|}
\hline Unit & Topic & $\begin{array}{c}\text { Bio/Life Science } \\
\text { Standards } \\
\text { Addressed }\end{array}$ & $\begin{array}{c}\text { CTE/Ag Standards } \\
\text { Addressed }\end{array}$ \\
\hline 1. & Careers in Animal Care & & $\begin{array}{l}\text { F1.3-12.4.3, F2.1-2.7, } \\
\text { F2.2-2.5, F2.2-1.3, F2.2- } \\
1.7, \text { F2.2-2.5, F2.4-1.1, } \\
\text { F2.4-2.3, F3.0, F7.0-7.6, } \\
\text { F8.3, F9.1, F9.2, F10.2, } \\
\text { F11.0 }\end{array}$ \\
\hline 2. & Laws and Animal Regulations & & $\begin{array}{l}\text { D1.4, D9.2, D9.3, D10.2, } \\
\text { D12.0, F1.3-12.2.3, F6.1, } \\
\text { F8.1, F8.2, F8.3, F9.4, } \\
\text { F11.0, FD8.3, }\end{array}$ \\
\hline 3. & HACCP Ethics Training & & $\begin{array}{l}\text { D6.5, D9.3, D9.4, D12.1, } \\
\text { F6.1, F6.2, F9.4, F11.0 }\end{array}$ \\
\hline 4. & $\begin{array}{l}\text { Animal Handling: Restraint, } \\
\text { Transport, Disposal and Harvest }\end{array}$ & & $\begin{array}{l}\text { D1.3, D1.4, D9.2, D9.4, } \\
\text { D12.1, D12.2, D12.3, } \\
\text { D12.4, D12.5, D12.6, } \\
\text { F1.3-12.2, F8.1, } \\
\text { F8.2,F11.0, FD8.1, } \\
\text { FD8.2 }\end{array}$ \\
\hline 5. & $\begin{array}{l}\text { Animal Behavior and } \\
\text { Observation }\end{array}$ & BI 1.d, BI 1.a & $\begin{array}{l}\text { D5.1, D9.1, D9.2, } \\
\text { D10.1, D11.3, F5.1, } \\
\text { F5.2, F5.3, F11.0 }\end{array}$ \\
\hline
\end{tabular}




\begin{tabular}{|c|c|c|c|}
\hline 6. & $\begin{array}{l}\text { Animal Health: Management, } \\
\text { Prevention, Housing }\end{array}$ & BI 1.1 & $\begin{array}{l}\text { D1.1, D1.2, D3.2, D6.4, } \\
\text { D9.2, D10.1, D11.3, } \\
\text { F2.2-2.3, F5.1, F5.2, } \\
\text { F5.3, F8.1, F11.0 }\end{array}$ \\
\hline 7. & Animal Breed Identification & & D5.0 \\
\hline 8. & $\begin{array}{l}\text { Introduction to Small \& } \\
\text { Specialty Animals }\end{array}$ & & $\begin{array}{l}\text { D10.1, D10.2, D11.1, } \\
\text { D11.2, D11.4, F11.0 }\end{array}$ \\
\hline 9. & $\begin{array}{l}\text { Basic Animal Nutrition: } \\
\text { Ruminant, Monogastric, Avian, } \\
\text { Equine }\end{array}$ & $\begin{array}{c}\text { BI 1.g, BI 1.e, BI 4.e, BI } \\
4 . f,\end{array}$ & $\begin{array}{l}\text { D2.2, D2.3, D2.4, D3.1, } \\
\text { D9.2, D10.1, D11.2, } \\
\text { D11.3, F11.0 }\end{array}$ \\
\hline 10. & Animal Anatomy/Physiology & $\begin{array}{c}\text { BI 1.j, BI 2.a, BI 9.a, BI } \\
\text { 9.b, BI 9.c, BI 9.d, BI } \\
\text { 9.e, BI 9.f, BI 9.g, BI 9.i, }\end{array}$ & $\begin{array}{l}\mathrm{D} 2.3, \mathrm{D} 2.4, \mathrm{D} 3.1, \mathrm{D} 3.2 \\
\mathrm{D} 4.0, \mathrm{~F} 11.0\end{array}$ \\
\hline 11. & $\begin{array}{l}\text { Introduction to Basic Pet } \\
\text { Grooming }\end{array}$ & & $\begin{array}{l}\text { D1.4,F2.4-1.1, F5.1, } \\
\text { F5.2, F5.3, F.6, F8.2, } \\
\text { F8.3, F9.5, F9.3, F11.0 }\end{array}$ \\
\hline 12. & $\begin{array}{l}\text { Introduction to Diseases and } \\
\text { Control, Non Living and Living } \\
\text { Agents, Spread of Disease, } \\
\text { Elimination, Protection Against, } \\
\text { First and Secondary Lines of } \\
\text { Defense }\end{array}$ & $\begin{array}{l}\text { BI 1.c, BI 10.a, BI 10.b, } \\
\text { BI 10.c, BI 10.d }\end{array}$ & $\begin{array}{l}\text { D6.1, D6.2, D6.3, D6.4, } \\
\text { D6.5, D6.6, D6.7, F2.1- } \\
\text { 2.3, F5.1, F11.0 }\end{array}$ \\
\hline 13. & $\begin{array}{l}\text { Common Diseases: Parasites, } \\
\text { Viral, Bacterial, Fungal, } \\
\text { Protozoan, Zoonotic, Nutritional } \\
\text { disease, Poison, Stress and } \\
\text { Heredity }\end{array}$ & $\begin{array}{l}\text { BI 1.c, BI 10.a, BI 10.b, } \\
\text { BI 10.c, BI 10.d }\end{array}$ & $\begin{array}{l}\mathrm{D} 2.2, \mathrm{D} 6.3, \mathrm{D} 6.6, \mathrm{~F} 2.4- \\
1.7, \mathrm{~F} 11.0\end{array}$ \\
\hline 14. & $\begin{array}{l}\text { Normal Values: Temperature, } \\
\text { Pulse, Breathing and } \\
\text { Respiration, Skin, Membranes, } \\
\text { Intestinal Discharge }\end{array}$ & BI 1.j, BI 10.a, & $\begin{array}{l}\text { D3.1, F4.0-4.6, F6.5, } \\
\text { F11.0 }\end{array}$ \\
\hline 15. & $\begin{array}{l}\text { Pharmacology: Route of } \\
\text { Administration, Measurement, } \\
\text { Storage, Size and Shape of } \\
\text { Needles and Syringes, Common } \\
\text { Drugs, Prescription } \\
\text { Requirements }\end{array}$ & BI 5.a & $\begin{array}{l}\text { F1.1-10.0, F1.1-13.0, } \\
\text { F1.1-15.0, F1.1-8.0, F4.0- } \\
4.6, \text { F6.2, F6.4, F6.5, } \\
\text { F6.6, F8.1-F8.3, F11.0 }\end{array}$ \\
\hline 16. & $\begin{array}{l}\text { Emergency Procedures: First } \\
\text { Responders Kit, Splint } \\
\text { techniques, Wound packing }\end{array}$ & & $\begin{array}{l}\text { D1.4,F4.0-4.6, F6.6, } \\
\text { F9.6, F11.0 }\end{array}$ \\
\hline 17. & $\begin{array}{l}\text { Common Surgical Procedures: } \\
\text { Aseptic technique, Surgical } \\
\text { preparation, Castration, Abscesses, } \\
\text { Dentistry, Suture technique }\end{array}$ & BI 1.a, BI 10.e & $\begin{array}{l}\text { F4.0-4.6,F6.4, F6.5, } \\
\text { F6.6, F8.1-F8.3, F11.0 }\end{array}$ \\
\hline 18. & $\begin{array}{l}\text { Laboratory Procedures: } \\
\text { Microscope use, Equipment } \\
\text { Identification, Collecting and } \\
\text { Handling Specimens }\end{array}$ & $\begin{array}{l}\text { BI 1.j, BI 1.c, BI 1.d, BI } \\
\text { 1.e, BI 1.f }\end{array}$ & $\begin{array}{l}\text { D1.4, D4.4, D6.1, D9.3, } \\
\text { F1.2-1.a, F1.2-1.c, } \\
\text { F1.2-1.d, F1.2-1.j, F1.2- } \\
\text { 1.m, F2.1-2.8, F2.2-1.1, } \\
\text { F2.2-1.2, F2.3-1.1, } \\
\text { F2.2-1.2, F2.2-1.3, }\end{array}$ \\
\hline & & & $\mathrm{F} 6.4, \mathrm{~F} 6.5, \mathrm{~F} 6.6$ \\
\hline 19. & $\begin{array}{l}\text { Reproductive Processes: } \\
\text { Gestation, Parturition, Artificial } \\
\text { insemination, Embryo transfer }\end{array}$ & $\begin{array}{l}\text { BI 2.a, BI 2.b, BI 2.d, BI } \\
\text { 2.e, BI 2.f, BI 2.g, BI 3.a, } \\
\text { BI 3.b, BI 3.c, BI 3.d }\end{array}$ & $\begin{array}{l}\text { D4.1, D4.2, D4.3, D4.4, } \\
\text { D4.5, D5.1, D5.2, D5.4 }\end{array}$ \\
\hline
\end{tabular}




\section{Ag Sales}

1. The role and organization of the Agribusiness

a. Agribusiness's place in California, United States, and the global economy

b. Types of agribusiness

c. The organization of an agribusiness

d. Managing the agribusiness

2. Models of management, organizations, and work.

a. Survey and exploration of careers in the Ag Business industry

b. Managerial work

c. The agriculture organization

d. Working in the agriculture organization

3. Goals in the agribusiness organization

a. The nature of goals

b. Multiple organizational goals

c. Managerial process of goal setting

d. Evaluation of goal attainment

4. The Decision Maker

a. Image formation

b. Behavioral decision methods

c. Quantitative decision methods

5. Financial Management and Control of Agribusiness

a. Overview of financial statements

b. Controlling and managing the agribusiness

c. Sources of financing

6. Communication in the Agribusiness Organization

a. The communication process

b. Communication and organization structure

c. Communication and the managerial process

7. Leadership

a. The nature of leadership

b. Factors that influence the leadership role

c. The role of the agriculture manager

8. Business Etiquette

a. Professional dress for men and women

b. Business conversation

c. Men and women as colleagues

d. Restaurant and table etiquette

e. Telephone etiquette

9. Marketing and Sales

a. Introduction to selling

b. Preparing to sell

c. Developing sales skills

d. Selling

e. Careers in Selling

f. Advertisements 
g. Marketing Plan

1. SWOT Analysis

2. Long Term and Short Term Goals

10. Planning in the Agribusiness

a. A model of planning

b. Designing plans

c. Organizing for planning

11. Ethics in Agriculture
a. Agriculture values
b. Agriculture ethics
c. Personal values

12. Agriculture Employee Motivation

a. Nature of human motivation

b. Equity in the workplace

c. Labor relations

13. Groups and Teams
a. The nature of groups
b. The character of groups
c. Teams and team building 


\section{Program Plan Part F}

\section{Program Completer Standards}

To insure the greatest possible standards of excellence in the Ridgeview Agriculture Program, the following areas must be completed in order for a student to be considered as a "Program Completer":
* 1.) A student must maintain a "grerage in all Agriculture course work.
2.) A student must be involved for four (4) years in the FFA Program.*
- $\quad$ The student must be proficient in Parliamentary Procedure.
- The student must have given a six minute speech or led a group discussion for 40 minutes on a topic relating to the FFA.
- They must have taken part in 16 FFA activities. $\sqrt{ } 5$ of these activities must be above the chapter level.
3.) A student must be involved in an expanding SOE Project for three years.*
*
The student must have earned or productively invested at least $\$ 1000.00$, or worked at least $\$ 00$ hours in excess of scheduled class time.

4.) Maintain an Agriculture Portfolio containing:

- Letter of Introduction

- Career Development Package.

$\sqrt{ } \quad$ Application for employment

$\sqrt{ }$ Letter of recommendation

$\sqrt{ }$ Resume

- Four work samples with summaries.

- Writing Sample.

- $\quad$ Supervised Occupational Experience Evaluation.

*This information must be verified in the student's FFA Recordbook. 


\section{Program Plan Part G}

\section{Description of Facilities and Major Equipment}

\section{Facility Description}

Construction on Ridgeview High School started in 1994 and was completed in 1995. The $\mathrm{Ag}$ Department utilizes five different areas on the campus.

1.) The $\mathrm{Ag}$ Classroom/ $\mathrm{Ag}$ Office currently gets the most use. The classroom has 35 student stations, student storage, ample material storage, eight computer network hubs as well as teacher use equipment (Computer, desk, filing cabinets). There is also a small office located in the back of the class for teacher use. Most classes (Agriculture Science I \& Ag Biology) are taught here.

2.) The Agriculture Technology Shop is next door to the classroom. It is a small shop with three storage areas, roll up doors and an area to paint small projects. Electrical outlets are lowered from the ceiling and an exhaust system is installed. Currently, the shop is being utilized as a campus storage area and has not been approved by the administration as a teaching laboratory. In the future, Introduction to Agriculture Technology shops may be taught there.

3.) The Ag Biology Laboratory is across the hall from the Ag Classroom. This lab, is the same lab that biology classes are currently being taught from. The lab is managed by the Science Department and used 1 period a day by the Ag Biology Class to perform laboratories to reinforce learning.

4.) The Agriculture Computer Lab is located in room 1217. It includes thirty five (35) IBM (Pentium II) Compatible machines with sound cards, five (5) Hewlett Packard Ink Jet Printers, a teacher station with an internal CD ROM, sound and video cards, a video projection Screen, and several software packages including: Windows 95, MicroSoft Office 97, Aldus Page Maker \& Photo Shop, California FFA Record Book, MicroSoft Internet Explorer and MicroSoft Front Page. All machines are linked to the schools main network and are able to access other computer networks (including the world wide web).

5.) To the south of the cafeteria, lies a $1 / 4$ acre fenced area, which will be used to house the greenhouse, plant propagation and horticultural area. The area should be completed by the year 2000 . 


\title{
Program Plan Part H
}

\author{
Ridgeview Agriculture Department \\ Five-Year Plan for Facility and Equipment \\ Acquisition Schedule
}

\section{$\underline{2014-2015}$}

Shade house

Move into larger classroom

Get approval for use of off-site farm

$\underline{2015-2016}$

Tables for the shade house

$2^{\text {nd }}$ teacher

Make improvements on off-site farm

2016-2017

Make improvements on off-site farm

Acquire new fair equipment

$\underline{2017-2018}$

New fair storage equipment area

Make improvements on off-site farm

$\underline{2018-2019}$

Get our own class set of chromebooks

Make improvements on off-site farm 
Program Plan Part I

\section{RIDGEVIEW AGRICULTURE DEPARTMENT CHART OF RESPONSIBILITIES 2014-2015}

\begin{tabular}{|l|c|c|}
\hline \hline A. COURSES & Potterton & \\
\hline 1. Agriculture Science & & \\
\hline a. AgEarth Science & $\times$ & \\
\hline b. AgBiology & $\times$ & \\
\hline c. Ver Soience & & \\
\hline d. Ag Sales & $\times$ & \\
\hline
\end{tabular}

\begin{tabular}{|c|c|c|}
\hline B. DEPARTHENT MANAGEMENT & Potterton & \\
\hline 1. Department Chairperson & $x$ & \\
\hline a. Agriculture Incentive Grant & $x$ & \\
\hline 1.) Ag Incentive Grant Application & $x$ & \\
\hline 2.) Ag Incentive Grant Expenditure Report & $x$ & \\
\hline 3.) Ag Incentive Grant Review Materials & $\mathrm{x}$ & \\
\hline 4.) AgDepartment Program Plan & $x$ & \\
\hline b. AgDepartment Purchase Drders & $x$ & \\
\hline 1.) Ag Incentive Grant Account & $x$ & \\
\hline 2.J Perkins Grant Acoount & $x$ & \\
\hline 3.J FFA ASB account & $x$ & \\
\hline c. Agriculture Curriculum & $x$ & \\
\hline d. Agriculture Department Parents Club & $x$ & \\
\hline 1.) Parents Club Meetings & $x$ & \\
\hline a.) AgDepartment Report & $x$ & \\
\hline b.) FFA Advisor Report & $x$ & \\
\hline & Potterton & \\
\hline c.) FFA Member Participation & $x$ & \\
\hline e. AgDepartment Advisory Committee & $x$ & \\
\hline 1.) Advisory Committee Meetings & $\mathrm{x}$ & \\
\hline 2.) Advisory Committee Meeting Announcement & $x$ & \\
\hline 3.) Advisory Committee Meeting Agenda & $x$ & \\
\hline 4.) Advisory Committee Meeting Minutes & $x$ & \\
\hline f. Agriculture Department Meetings & $\mathrm{x}$ & \\
\hline 1.) Agriculture Department Meeting Agenda & $x$ & \\
\hline 2.) Agriculture Department Meeting Minutes & $x$ & \\
\hline 3.) Agriuclture Department Calendar & $x$ & \\
\hline
\end{tabular}




\begin{tabular}{|c|c|c|}
\hline 4.) Agriculture Department Records & $x$ & \\
\hline 5.) Agriculture Department Supply Requisitions & $x$ & \\
\hline \multicolumn{3}{|l|}{ g. Miscellaneous } \\
\hline 1.) Agriculture Teacher Inservice Record & $x$ & \\
\hline 2.) Agriculture Teacher Summer Hours & $x$ & \\
\hline 2. FFA Advisor & $x$ & \\
\hline a. Student Data Sheets & $x$ & \\
\hline 1.) Agriculture Department State R-2Report & $x$ & \\
\hline a.) Graduate Follow- $\bigsqcup_{p}$ Survey & $x$ & \\
\hline 2.) Ag Department State FFA Roster Update & $x$ & \\
\hline b. Activity Requests & $x$ & \\
\hline 1.) Transportation Requests & $x$ & \\
\hline 2.J Facility Requests & $x$ & \\
\hline 3.) Master Calendar Requests & $x$ & \\
\hline 4.) Fundraiser Requests & $x$ & \\
\hline \multicolumn{3}{|l|}{ 3. Miscellaneous } \\
\hline a. Qther Grant Applications & $x$ & \\
\hline b. Qther Scholarship Applications & $x$ & \\
\hline \multicolumn{3}{|l|}{ 4. Equipment and Facilities } \\
\hline a. Garden & $x$ & \\
\hline 1.) Maintenance and Repair of Facility & $x$ & \\
\hline b. Agriculture Department Storage Facility $(\mathrm{H}-1)$ & $x$ & \\
\hline 1.) Agriculture Department Vehicle & $x$ & \\
\hline a.) Vehicle Maintenance and Service & $x$ & \\
\hline 2.) Agriculture Department Stock Trailer & $x$ & \\
\hline 3.) Agriuclture Department Tractor & $x$ & \\
\hline a.) Agriculture Department Equipmentilmpliments & $x$ & \\
\hline b. Degrees & $x$ & \\
\hline 1.) Greenhand Degree Applications & $x$ & \\
\hline 2.) Chapter Degree Applications & $x$ & \\
\hline 3.) State Degreee Applications & $x$ & \\
\hline 4.) American Degree Applications & $x$ & \\
\hline \multicolumn{3}{|l|}{ c. Leadership Conferences } \\
\hline 1.) Chapter Officer Leadership Conference & $x$ & \\
\hline 2.) GreenhandDegree Conference & $x$ & \\
\hline 3.) Made For Excellence Conference & $x$ & \\
\hline 4.) Advanced Leadership Conference & $x$ & \\
\hline 5.) State FFA Leadership Conference & $x$ & \\
\hline d. FFA Chapter Meetings & $x$ & \\
\hline
\end{tabular}




\begin{tabular}{|c|c|c|}
\hline 1.) FFA Chapter Officers & $X$ & \\
\hline a.) Chapter Officer M eetings & $X$ & \\
\hline 1.) Chapter Officer A pplications & $X$ & \\
\hline 2.) Chapter Officer Retreat & $X$ & \\
\hline b.) Chapter Program Of Work & $X$ & \\
\hline c.) Chapter Point Awards Program & $X$ & \\
\hline d.) Chapter Recruitment Program & $X$ & \\
\hline 2.) Chapter Banquets & $X$ & \\
\hline a.) Banquet Awards & $X$ & \\
\hline b.) Banquet Program & $X$ & \\
\hline c.) Banquet Slide Show & $X$ & \\
\hline 3.) Community Service & $X$ & \\
\hline 4.) Fundraisers & $X$ & \\
\hline 5.) Miscellaneous & & \\
\hline a.) FFA Welcome BBQ & $X$ & \\
\hline b.) FFA Week Activites & $X$ & \\
\hline c.) FFA Reward Trip (End of the Year) & $X$ & \\
\hline d.) Ag Expo (Tulare Farm Show) & $X$ & \\
\hline e.) FFA Jacket Orders & & \\
\hline f.) FFA State Finals Judging Contest Trip & $X$ \\
\hline
\end{tabular}

\begin{tabular}{|l|c|c|}
\hline D. SUPERVISED AG RICULTURAL EXPERIENCE & POTTERTON \\
\hline 1. SAE Project Supervision & $\mathrm{X}$ & \\
\hline a. Livestock & $\mathrm{X}$ & \\
\hline 1.) Beef Cattle & $\mathrm{X}$ & \\
\hline 2.) Dairy Cattle & $\mathrm{X}$ & \\
\hline 3.) Goats & $\mathrm{X}$ & \\
\hline 4.) Sheep & $\mathrm{X}$ & \\
\hline 5.) Swine & $\mathrm{X}$ & \\
\hline b. Small Animals & $\mathrm{X}$ & \\
\hline 1.) Poultry & $\mathrm{X}$ & \\
\hline 2.) Rabbits & $\mathrm{X}$ & \\
\hline c. Agriculture Mechanics & $\mathrm{X}$ & \\
\hline d. Ornamental Horticulture & $\mathrm{X}$ & \\
\hline e. Agricultural Work Experience & $\mathrm{X}$ & \\
\hline 2. Fairs and Shows & & \\
\hline a. SAE Project Supervision and Transportation & $\mathrm{X}$ & \\
\hline b. Student Supervission and Transportation & $\mathrm{X}$ & \\
\hline c. Chapter Supply and Equipment Transportation & $\mathrm{X}$ & \\
\hline
\end{tabular}




\begin{tabular}{|l|l|l|}
\hline d. Fair and Show Entries and Fees & $X$ & \\
\hline e. Fair and Show Entry Tagging & $X$ & \\
\hline 1.) Kern County Fair & $X$ & \\
\hline 2.) Desert Empire Fair & $X$ & \\
\hline 7.) M iscelaneous Other Exhibits & $X$ & \\
\hline 3. SAE Project Proficiencies & & \\
\hline a. Chapter Proficiencies & $X$ & \\
\hline 1.) Chapter Proficiency Scoring & $X$ & \\
\hline b. Sectional Proficiencies & $X$ & \\
\hline 1.) Sectional Proficiency Scoring & $X$ & \\
\hline c. Regional Proficiencies & $X$ & \\
\hline 1.) Regional Proficiency Scoring & $X$ & \\
\hline d. State Proficiencies & $X$ & \\
\hline 1.) State Proficiency Scoring & $X$ & \\
\hline e. National Proficiencies & $X$ & \\
\hline 1.) National Proficiency Scoring & $X$ & \\
\hline
\end{tabular}

\begin{tabular}{|l|c|c|}
\hline E. JUDG ING TEAMS & Potterton & \\
\hline 1. Leadership Teams & & \\
\hline a. FFA Opening and Closing Ceremonies & & \\
\hline 1.) Officer Opening and Closing Ceremonies & $X$ & \\
\hline 2.) Open Opening and Closing Ceremonies & $X$ & \\
\hline 3.) Freshmen Opening and Closing Ceremonies & $X$ & \\
\hline b. FFA Public Speaking & & \\
\hline 1.) FFA Creed Speakers & $X$ & \\
\hline 2.) Prepared Public Speaking & $X$ & \\
\hline 3.) Job Interview & $X$ & \\
\hline \multicolumn{3}{|l|}{ POTTERTON } \\
\hline
\end{tabular}

\begin{tabular}{|l|l|l|}
\hline 5. Miscellaneous & & \\
\hline a. Banking & $X$ & \\
\hline b. Coops & $X$ & \\
\hline c. Novice Records & $X$ & \\
\hline e. Best Informed Greenhand & $X$ & \\
\hline
\end{tabular}


Program Plan Part J

Program of Work. See masters project. 


\section{Program Plan Part K}

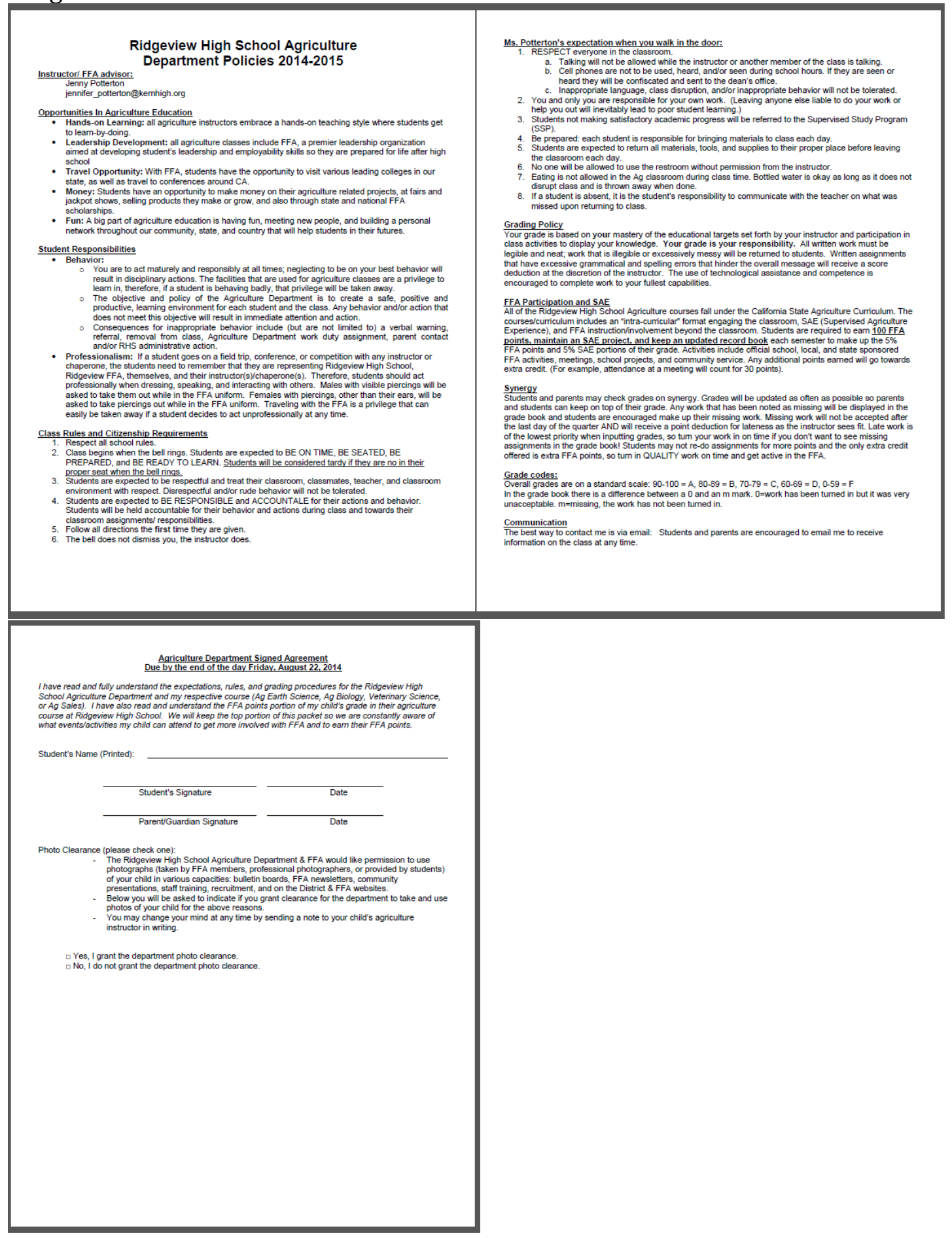




\section{Program Plan Part L}

A program completer of the Ridgeview High School is a student who has taken 4 years of coursework in Agriculture Science, including their senior year. Program completers will receive a sash and be recognized at the high school graduation ceremony. 


\section{Program Plan Part M}

R2 Teacher Information

Ridgeview HS,Bakersfield

Year: 2014

\begin{tabular}{|l|l|l|l|l|c|c|c|c|c|c|c|}
\hline $\begin{array}{l}\text { Last } \\
\text { Name }\end{array}$ & $\begin{array}{l}\text { First } \\
\text { Name }\end{array}$ & MI & Gender & Ethnicity & $\begin{array}{c}\text { Total } \\
\text { Years } \\
\text { Teaching } \\
\text { Ag. }\end{array}$ & Credential Type & $\begin{array}{c}\text { 9-Month } \\
\text { Salary }\end{array}$ & $\begin{array}{c}\text { Extended } \\
\text { Contract } \\
\text { Stipend }\end{array}$ & $\begin{array}{c}\text { FFA } \\
\text { Stipend }\end{array}$ & $\begin{array}{c}\text { Department } \\
\text { Head } \\
\text { Stipend }\end{array}$ & $\begin{array}{c}\text { SOE } \\
\text { Period }\end{array}$ \\
\hline Potterton & Jennifer & R & Female & White & 3 & $\begin{array}{c}\text { Agriculture } \\
\text { Specialist }\end{array}$ & 53936 & 10330 & 0 & 0 & $\mathrm{~N}$ \\
\hline
\end{tabular}

\begin{tabular}{|l|l|l|l|l|l|}
\hline Potterton, Jennifer \\
\hline Schedule & Period & Beginning Time & Course Title & Enrollment & Type \\
\hline 1 & 1 & 0825 & ag sales & 37 & Ag Bus Mgt \\
\hline 1 & 3 & 1030 & ag biology & 31 & Ag Biology \\
\hline 1 & 5 & 1206 & ag earth science & 36 & Agriscience I \\
\hline 1 & 6 & 0115 & ag earth science & 33 & Agriscience I \\
\hline 1 & 7 & 0220 & ag earth science & 30 & Agriscience I \\
\hline
\end{tabular}

Printed: 7/2/2015 11:38:09 AM 


\section{Program Plan Part N}

Ralph Mendes - KHSD Ag Coordinator

Mike Poncetta - Partner-Poncetta Farms Inc.

Kristi Harrer - PCA/Agronomist

Ross Keith - Maintenance/Facility Supervisor at Get Bus

Chris Persons - School Administrator 


\section{Program Plan Part 0}

\section{Ridgeview FFA Advisory Committee Meeting Minutes}

\section{$9 / 3 / 14$}

- Welcome, in attendance: Ross Keith, Ralph Mendes, Michael Poncetta, Jenny Potterton

- 2014 Fair Update: Kern County Fair numbers: 13 pig exhibitors, 15 head of pigs; 2 sheep exhibitors, 3 head of sheep; 2 rabbit exhibitors, 2 rabbits; 3 still exhibit exhibitors with gardenrelated projects. Target for next year: 5 goats, 2 dairy cattle (talk to Julie Beechinor about financing projects, Mike will help find farmers). Desert empire Fair numbers: 1 sheep

- Garden update: working on getting a shade house, Ralph suggests sterilizing the area to reduce weeds, and putting gravel as flooring. Need to get tables, Ralph will look into a vendor, also could have Matt Riley's students at Mira Monte build tables

- School farm update: gave Ross the insurance, will be working with Chris Persons to get everything worked out so we can use it this summer

- FFA update: Welcome AGtivities had over 70 kids in attendance, 1 American Degree, started a leadership team. Ralph suggests starting a Greenhand officer Team

- 2014-2015 Planned Calendar: went over planned calendar for the year and how to execute it. Ralph suggests: starting BIG early, will help coach banking, use the 3 greenhands on leadership team as creed competitors, publicize an include administration a community service projects, Ross' Dad $n$ the board at the homeless shelter

- Advisory Committee structure, etc.: we expect a visit from regional supervisor, Charles Parker in December (4th?), Ross volunteered to be committee chair and will be there to meet with Mr. Parker, review AIG application, need to review checklist next meeting

- Suggested Committee Members: Leticia Davidson (veterinarian), and Dr. Piokin (Panama equine hospital), Andrea/Tom Price (Bugni's owners, Mike will work on contacting them), Rebecca Bullier (Grimway, 699-2384)

- Questions, Comments, Suggestions: work with administration to get a second teacher

- Next meeting: Nov. 5, 6pm at Molly J's 
Ridgeview FFA Advisory Committee Meeting Agenda

5/19/14 6:06-6:47

- Welcome: present-Ross Keith, Jenny Potterton

- End of the Year banquet review-banquet was very successful, about 70 kids and most families came, which is over $1 / 2$ the membership

- 2014 Fair Update-current numbers: 1-beef, 3-sheep, 1-goat, 1-chicken, 5/6-rabbits, 10-pigs. Up from 7 exhibitors last year.

- Garden update-no greenhouse because of state regulations, trying to get shade. Ross suggested triangle shades from Costco and corrugated plastic over the beds

- School farm-trying to push new administration to get information so we could use a farm next year.

- 2014-2015 Planned Calendar-similar to last year, additions include: winter banquet (maybe use as a fundraiser), judging team field days, team building boot camp)

- Fundraiser ideas- it's still unknown whether or not we will have the Ag Incentive Grant next year. Last year we received about $\$ 7000$ from AIG, so we are pushing fundraising. We have a panda express fundraiser Thursday and will be getting Cruz Thru carwash coupons soon, also we do the poinsettias (didn't make as much as we could have this year, but now have better marketing ideas). Other ideas: graduation gladiolas from the garden, wreaths from grapevine or Christmas tree trimmings, mixed bags, tahoe joes/outback, sarulash with a better location (fresh and easy, kern schools parking lot). Having students write a paper about what ag ed means to them to send in to help encourage the funding of the AIG.

- Suggested Committee Members: try the 2 earlier proposed:

- Ryan Fechon, Tejon Ranch Manager 340-5979

- Rebecca Bulliec Grimway 699-2384

Also try:

- Bugni's owners

- Vet?

- Drew Parker, Assistant principal

- Questions, Comments, Suggestions- new classroom next year, bigger. 


\begin{tabular}{|c|c|c|c|c|c|}
\hline & & & A & $B$ & $\mathrm{C}$ \\
\hline Line & $\begin{array}{c}\text { Acct. } \\
\text { No. }\end{array}$ & Classification & $\begin{array}{l}\text { Description of Item for } \\
\text { Which Funds Will be } \\
\text { Expended }\end{array}$ & \begin{tabular}{|c|} 
Incentive \\
Grant Funds
\end{tabular} & $\begin{array}{l}\text { Matching } \\
\text { Funds }\end{array}$ \\
\hline 1 & 4000 & Books \& Supplies & & $6,336.00$ & $6,336.00$ \\
\hline 2 & & & Subtotal for 4000 & $\$ 6,336.00$ & $\$ 6,336.00$ \\
\hline 3 & \multirow[t]{6}{*}{5000} & \multirow{6}{*}{$\begin{array}{l}\text { Services and Other Operating } \\
\text { Expenses such as: Services of } \\
\text { Consultants, Staff Travel, and } \\
\text { Conference; Rentals, Leases, and } \\
\text { Repairs; Bus Transportation }\end{array}$} & 1. Travel and Conf & $1,000.00$ & $1,000.00$ \\
\hline 4 & & & 2. & & \\
\hline 5 & & & 3. & & \\
\hline 6 & & & 4. & & \\
\hline & & & 5. & & \\
\hline 7 & & & 6. & & \\
\hline 8 & & & Subtotal for 5000 & $\$ 1,000.00$ & $\$ 1,000.00$ \\
\hline 9 & \multirow[t]{5}{*}{6000} & \multirow{5}{*}{$\begin{array}{l}\text { Capital Outlay: Includes Sites and } \\
\text { Improvements of Sites; Buildings } \\
\text { and Improvement of Buildings; } \\
\text { Equipment }\end{array}$} & 1. & & \\
\hline 10 & & & 2. & & \\
\hline 11 & & & 3. & & \\
\hline & & & 4. & & \\
\hline 12 & & & 5. & & \\
\hline 13 & & & Subtotal for 6000 & $\$ 0.00$ & $\$ 0.00$ \\
\hline 14 & & & $\begin{array}{l}\text { Total for } 4000-6000 \\
\text { Lines } 2,8,13\end{array}$ & $\$ 7,336.00$ & $\$ 7,336.00$ \\
\hline
\end{tabular}

TOTAL 2015-16 Incentive Grant Allocation: 


\section{Program Plan Part Q}

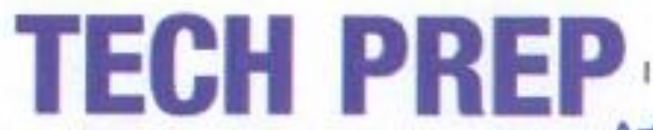

B A KERSFIELD COLLEGE Articulated Courses by BC Career Pathway

\begin{tabular}{|c|c|c|c|c|c|c|c|c|c|c|c|c|c|c|c|c|c|c|}
\hline COURBE RAME and DE SCFIPTIOA & $\frac{\pi}{3}$ & $\frac{y}{2}$ & $\begin{array}{l}\text { o } \\
\frac{1}{3} \\
\end{array}$ & 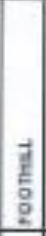 & $\begin{array}{l}\frac{z}{2} \\
\frac{5}{5} \\
\frac{5}{2} \\
\frac{2}{2}\end{array}$ & 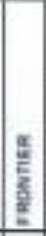 & 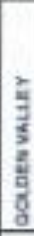 & $\begin{array}{l}9 \\
\frac{9}{2} \\
2 \\
0 \\
0\end{array}$ & 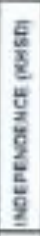 & $\begin{array}{l}\frac{b}{3} \\
\frac{3}{5} \\
\frac{z}{2}\end{array}$ & $\frac{Z}{\frac{Z}{E}}$ & 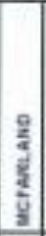 & $\begin{array}{l}\frac{5}{2} \\
\frac{3}{8} \\
\frac{5}{2} \\
\frac{5}{2}\end{array}$ & $\frac{z}{\frac{z}{2}}$ & $\frac{8}{\frac{y}{2}}$ & $\frac{3}{8}$ & $\frac{\frac{5}{2}}{\frac{5}{5}}$ & $\begin{array}{l}8 \\
\frac{1}{1} \\
\end{array}$ \\
\hline 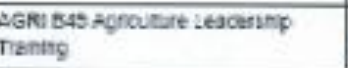 & - & - & - & 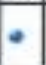 & & $\bullet$ & - & - & & - & & - & $\bullet$ & - & & $\bullet$ & - & - \\
\hline AGES BS2 Economice in Agroulthuse & & $\cdot$ & & - & & - & & $\bullet$ & & $\bullet$ & $\bullet$ & & $\bullet$ & $\bullet$ & $\bullet$ & & & $\bullet$ \\
\hline ANSC BI wes B Aanial scerce & - & & - & $\bullet$ & - & $\bullet$ & $\cdot$ & 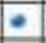 & & $\bullet$ & & 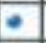 & & & & $\bullet$ & & - \\
\hline CMP S BI Princelet ef Coop Procusticn & & & $\bullet$ & 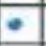 & 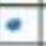 & & & $\cdot$ & & $\bullet$ & & - & & $\bullet$ & & & - & \\
\hline FORE BI rtrosuation Do Furestry & & & & & & & & & & & & & & & & & & \\
\hline 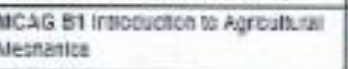 & - & - & & - & & & $\bullet$ & $\bullet$ & & - & & - & • & & & & & $\bullet$ \\
\hline 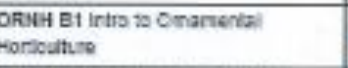 & 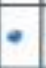 & - & - & & - & & & - & & $\bullet$ & & $\bullet$ & & & - & - & $\bullet$ & - \\
\hline 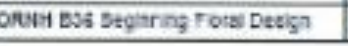 & $*$ & $\bullet$ & & & & & & & & - & & & & & & & & $\bullet$ \\
\hline
\end{tabular}


Program Plan Part R

Students are surveyed at time of graduation at the end of the school year.

Program Plan Part S

\section{S. List of Active Placement Sites}

The following is a list of places students are currently interning
or working at, or are places we could potentially send students.

Mebane Ranch

Famosa Livestock Yard

Rancho Rio

Grisedale Ranch

Granite Oaks Ranch

Granite Station Feed Store

KC Sheep

RKA Rabbitry

Fur and Feathers

Michelle's Custom Cutting

Velo's Equine Training

Fred C. Gilbert Feed Company

Jim's Honey Farm LIC

Olive Drive Veterinarian Hospital

North of the River Veterinarian Hospital

Other Glennville/Woody Ranches

\section{Program Plan Part T}

The primary recruitment that occurs for the program is during Freshman Orientation. At this, I briefly speak to the incoming students and their parents. We also set up a booth where the current students are able to talk to potential students. At the booth we had a board of pictures, flowers from our garden, awards, brochures, and a card to contact me about fair information. Students also walked around with the brochure. I created the brochure this year and made sure to focus on including information about credits earned and how ag fits with the rest of their courses.

Within the school, we hosted daily lunch activities, dress up days, and a staff and member breakfast during FFA week. Also, students are included in the daily announcements to celebrate successes within our chapter. 


\section{Program Plan Part U INCENTIVE GRANT IN-SERVICE ACTIVITIES DOCUMENTATION \\ CRITERIA 4.B School Year 2014-15 School Ridgeview}

Based on the previous year's record, every agriculture teacher, teaching at least $1 / 2$ time agriculture, attends a minimum of four of the following professional development activities:

Qualified and Competent Personnel

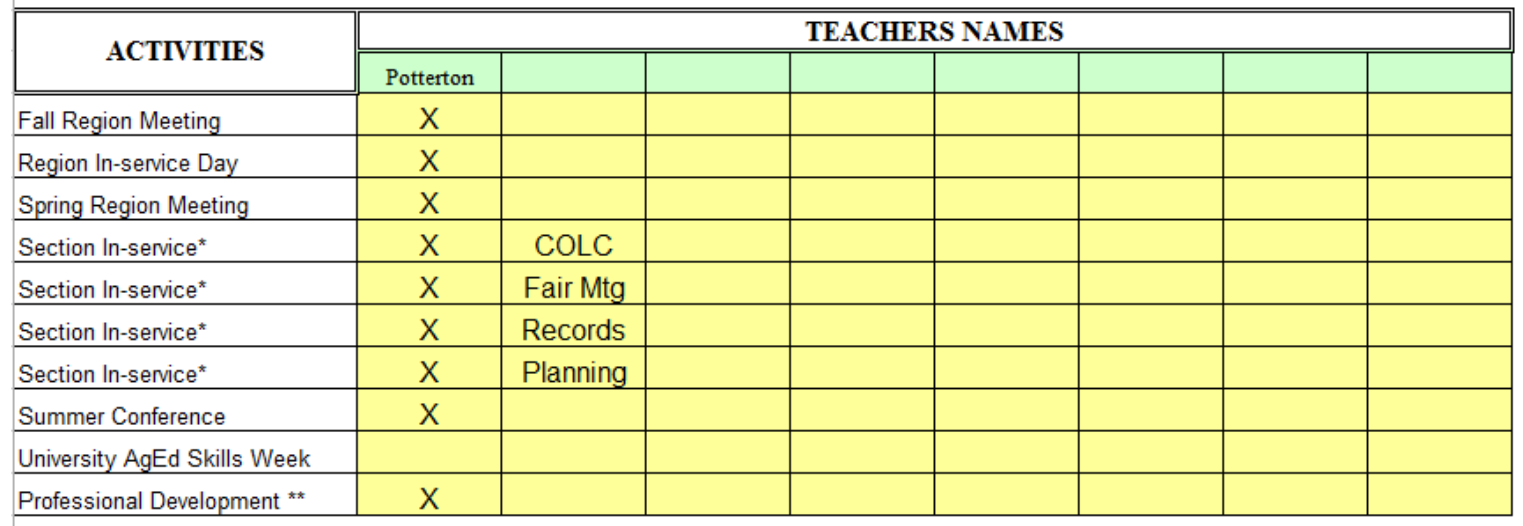

* Four Section In-service Meetings equals one Professional Development Activity

** Can utilize a maximum of two other "Agriculturally Related" Professional Development activities than those listed above. Explain the Professional Development:

1 Professional Learning Community--Science PLC

2 Ridgeview Summer Teacher Institute

3 Google Conference both district and Google

4 New Professional-Cal Poly

5 
Supporting Completion

Materials P: Advisory

Committee Agendas \&

Minutes

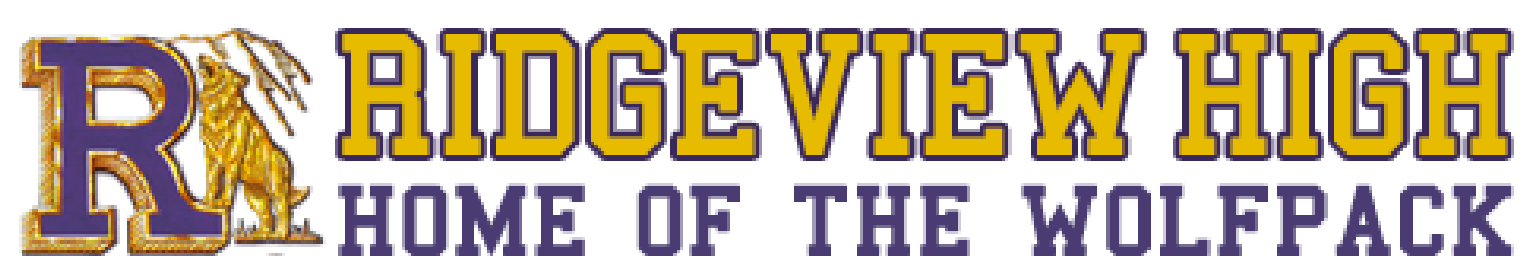




\section{P. Advisory Committee Agendas \& Minutes}

Supporting Completion Material Attached 
Ridgeview FFA Advisory Committee Meeting Agenda

$9 / 3 / 14$

- Welcome

- 2014 Fair Update

- Garden update

- School farm update

- FFA update

- 2014-2015 Planned Calendar

- Advisory Committee structure, etc.

- Suggested Committee Members

- Questions, Comments, Suggestions 


\section{Ridgeview FFA Advisory Committee Meeting Minutes}

\section{$9 / 3 / 14$}

- Welcome, in attendance: Ross Keith, Ralph Mendes, Michael Poncetta, Jenny Potterton

- 2014 Fair Update: Kern County Fair numbers: 13 pig exhibitors, 15 head of pigs; 2 sheep exhibitors, 3 head of sheep; 2 rabbit exhibitors, 2 rabbits; 3 still exhibit exhibitors with gardenrelated projects. Target for next year: 5 goats, 2 dairy cattle (talk to Julie Beechinor about financing projects, Mike will help find farmers). Desert empire Fair numbers: 1 sheep

- Garden update: working on getting a shade house, Ralph suggests sterilizing the area to reduce weeds, and putting gravel as flooring. Need to get tables, Ralph will look into a vendor, also could have Matt Riley's students at Mira Monte build tables

- School farm update: gave Ross the insurance, will be working with Chris Persons to get everything worked out so we can use it this summer

- FFA update: Welcome AGtivities had over 70 kids in attendance, 1 American Degree, started a leadership team. Ralph suggests starting a Greenhand officer Team

- 2014-2015 Planned Calendar: went over planned calendar for the year and how to execute it. Ralph suggests: starting BIG early, will help coach banking, use the 3 greenhands on leadership team as creed competitors, publicize an include administration a community service projects, Ross' Dad $\mathrm{n}$ the board at the homeless shelter

- Advisory Committee structure, etc.: we expect a visit from regional supervisor, Charles Parker in December ( $4^{\text {th }}$ ?), Ross volunteered to be committee chair and will be there to meet with $\mathrm{Mr}$. Parker, review AIG application, need to review checklist next meeting

- Suggested Committee Members: Leticia Davidson (veterinarian), and Dr. Pipkin (Panama equine hospital), Andrea/Tom Price (Bugni's owners, Mike will work on contacting them), Rebecca Bullier (Grimway, 699-2384)

- Questions, Comments, Suggestions: work with administration to get a second teacher

- Next meeting: Nov. 5, 6pm at Molly J's 


\section{Ridgeview FFA Advisory Committee Meeting Agenda}

$11 / 5 / 14$

- Welcome

- 2014 Fair Results

- Garden update

- School farm update

- FFA update

- On Site Ag Incentive Grant Review

- Suggested Committee Members

- Questions, Comments, Suggestions 


\section{Ridgeview FFA Advisory Committee Meeting Minutes}

\section{$11 / 5 / 14$}

- Welcome, in attendance Ross Keith and Jenny Potterton

- 2014 Fair Results: Reserve champion AOB lamb, good placings in the hog barn, still exhibits judges choice award and champion vegetable. Already have increased interest for next year, have some students doing cattle projects

- Garden update: shade house Is here, applied for PG\&E grant for gravel, weed cloth, tables, an planting supplies

- Working on a grant for chromebooks for the ag department, AIG specialized grant. Ross's mom may be able to help with the application

- School farm update: haven't heard anything from the administration on what there is left to do for approval, Ross is going to talk to Mr. Persons about it

- FFA update: opening and closing is coming up, planning on taking 6 teams. Public speakers: 3 prepared, 3 extemp, 3 job interview, 2 creed, 1 impromptu. Ross offered to help with job interview, his mom may be able to proof read the speeches.

- On Site Ag Incentive Grant Review: the on-site review is on December 4 at $1 \mathrm{pm}$. Ross will be in attendance. Went over the AIG checklist. We meet most criteria except 10 and 11 . Plan to schedule a meeting between Ross, Ralph Mendes, and Jenny before the review to make sure we know what to expect. Plan out what we need money for.

- Suggested Committee Members: continue trying to recruit the people mentioned at the last meeting

- Questions, Comments, Suggestions: Spoke about how to get better meeting attendance: maybe do an online meeting forum, don't plan dates so far in advance, suggest 3 dates and let the committee members choose 


\section{Supporting Completion \\ Materials Q: Student \\ Program Plan}

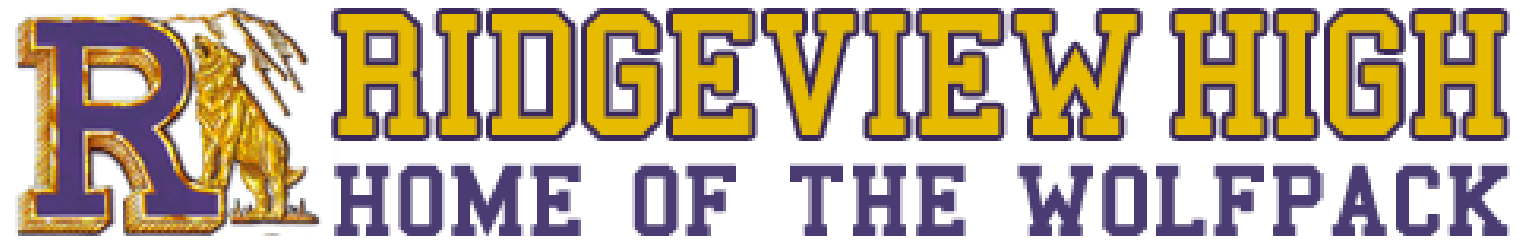




\section{Q. Student Program Plan}

Supporting Completion Material Attached 


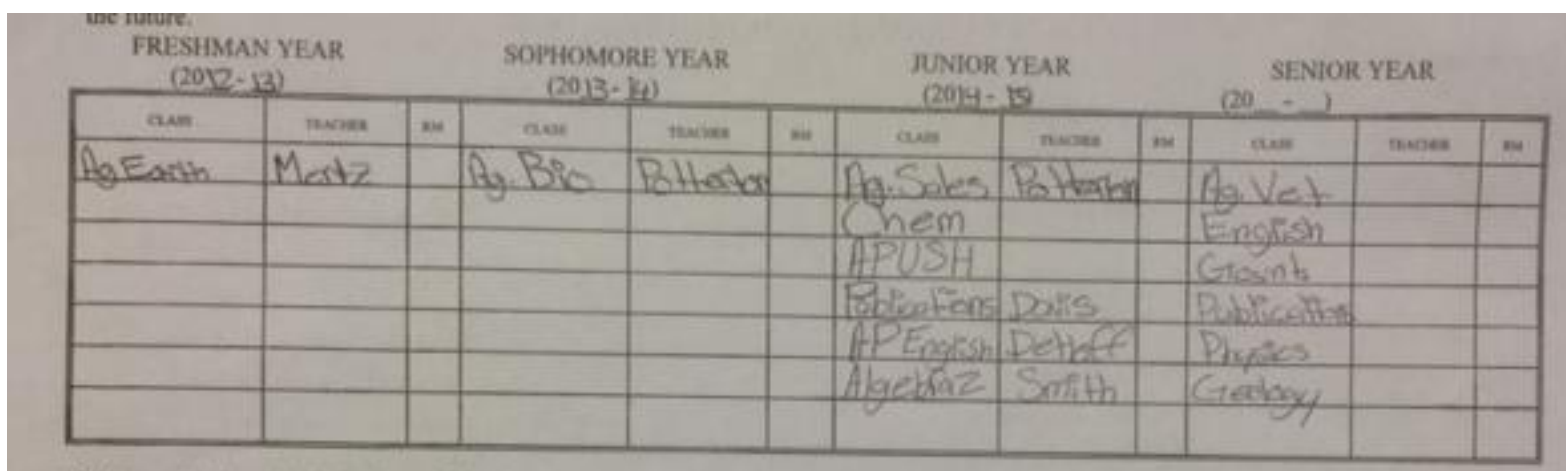

M. Supervised Practical Expericnce Plan (Project program should be related to career goal).

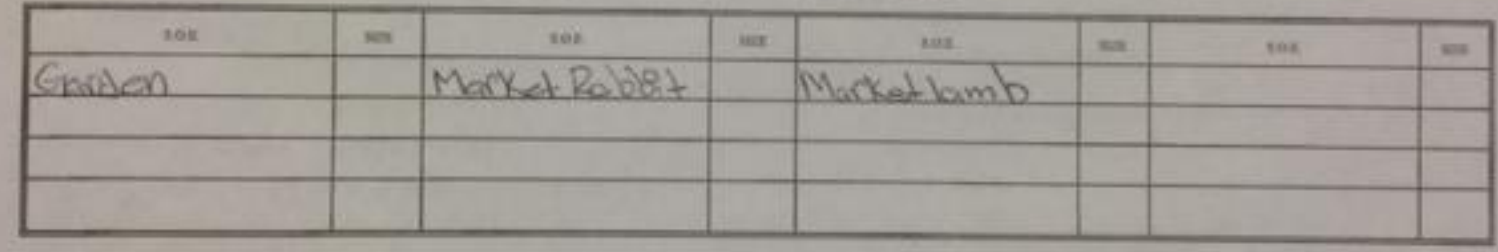

N. Planned Departmental Activities (FFA)

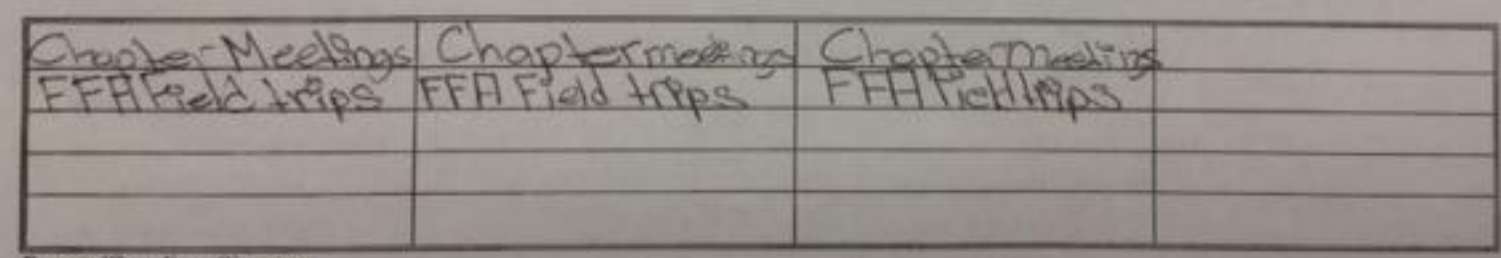

Parents Guardians Signature: 
Supporting Completion Materials R: Proficiency Standards

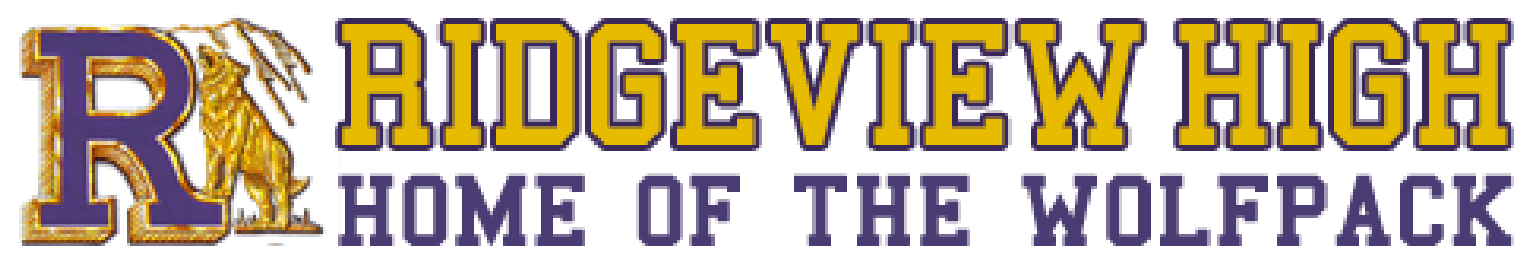




\section{R. Proficiency Standards}

Supporting Completion Materials Attached 


\section{Agriculture Earth Resources CP}

AFS $=$ Agriculture Foundation Standards

APS $=$ Agriculture Pathway Standards

California Adopted Earth Science Standards

\begin{tabular}{|c|c|}
\hline Topic & Standards Addressed \\
\hline California Agriculture & APS C1.1-6 \\
\hline Scientific Process & APS C13.1-3, AFS 1.2a-m, \&E 1. a-h \\
\hline $\begin{array}{l}\text { Plate Tectonics: ocean floor features, } \\
\text { plate boundaries, rocks }\end{array}$ & AFS 4.4, ES 3.a-d \\
\hline FFA & $\begin{array}{l}\text { AFS } 1.1(10.0) \text {, AFS } 2.1-4, \text { AFS } 7.0-6 \text {, AFS } \\
9.0-6 \text {, AFS } 10.1\end{array}$ \\
\hline $\begin{array}{l}\text { Plate Tectonics: earthquakes, } \\
\text { volcanoes }\end{array}$ & ES 3.d E \\
\hline Record Keeping & AFS 1.1(10.0), APS C $3.1-4$, AFS 10.3 \\
\hline California Geology & APS C10.1, ES 9. a-d \\
\hline Agriculture Resources & APS C2.1-5, ES 9 9-6 \\
\hline Bio-Geochemical Cycles & APS C11.5-6, ES 7. a-d \\
\hline Earth's Atmosphere & APS C10.3, ES 8. a-d \\
\hline Energy and Agriculture & APS E6.1\&4, ES A. b-6 \\
\hline Heating the Earth's Surface & ES 5. a-d \\
\hline Earth's Climate & APS E2.1-6, APS F2.4, ES 6. a-d \\
\hline Astronomy & ES 1.a-f \& 2.a-d \\
\hline $\begin{array}{l}\text { Soils: formation, texture, structure, } \\
\text { erosion, and conservation of }\end{array}$ & APS E3.2\&4 \\
\hline Supervised Agriculture Experience & $\begin{array}{l}\text { APS C4.1-5, C6.1-2, C8.1-3, C9.1-5, C11.1, } \\
\text { AFS } 10.2\end{array}$ \\
\hline $\begin{array}{l}\text { Agricultural Careers and Development } \\
\text { Events }\end{array}$ & AFS $3.1-6$, AFS $5.0-3$, AFS 11.0 \\
\hline
\end{tabular}


Agriculture Biology:

\begin{tabular}{|c|c|c|}
\hline Topic & $\begin{array}{c}\text { California } \\
\text { State Standards }\end{array}$ & $\begin{array}{c}\text { CTE/Agriculture } \\
\text { Standards }\end{array}$ \\
\hline $\begin{array}{l}\text { Scientific } \\
\text { Process }\end{array}$ & $\begin{array}{l}\text { I\&E c,d,f,j,l; Bio } \\
4 \mathrm{e}, 5 \mathrm{a}, 9 \mathrm{a}\end{array}$ & $\begin{array}{l}\text { 1.a,c,d,f,j,l,m } \\
\mathrm{C} 13.0 ; \mathrm{C} 13.1 ; \mathrm{C} 13.2 ; \mathrm{C} 13.3\end{array}$ \\
\hline Ecology & Bio 6a-f & $\begin{array}{l}\text { C2.1; C2.3; G1.0; G1.4; } \\
\text { D7.0; D7.2; E1.0 }\end{array}$ \\
\hline $\begin{array}{l}\text { Cellular Structure } \\
\text { and Process }\end{array}$ & Bio $1 c, e, f, g, h, l, j, 2 a$ & $\begin{array}{l}\text { C5.0; C5.1; C5.2; C5.3; C5.4 } \\
\text { C11.5; C11.4; C11.0 }\end{array}$ \\
\hline Genetics & $\begin{array}{l}\text { Bio 1d, 2a-g, 3a-b, 4a- } \\
\text { e, 5a-c, 7b }\end{array}$ & $\begin{array}{l}\text { C7.0; D5.0; D5.4; D5.5; } \\
\text { C3.3; C3.4 }\end{array}$ \\
\hline Evolution & Bio 7a-d, 8a-e & $\mathrm{C} 4.1 ; \mathrm{C} 4.2$ \\
\hline $\begin{array}{l}\text { Human Anatomy } \\
\text { and Physiology }\end{array}$ & Bio 9b-e, 10a-e & C6.0; C11.5; \\
\hline $\begin{array}{l}\text { Independent Lab } \\
\text { Based Projects, } \\
\text { CTE }\end{array}$ & $\begin{array}{l}\text { Bio 9a-l, } \\
\text { I\&Ea,b,c,d,f,g,k,l,m }\end{array}$ & $\begin{array}{l}\text { 2.0 - Communication:2.1, } \\
2.2,2.3,2.7,2.8 \text {, Writing: } \\
1.3,1.5,2.3,2.5,2.6 \text {, } \\
\text { Writing Strategies \& } \\
\text { Applications: } 1.6,2.6 \\
\text { Listening \& Speaking Skills: } \\
1.1,1.7 \text {, } \\
9.0 \text { - Leadership \& Team } \\
\text { Work: } 9.1,9.2,9.3 \text {, } \\
\text { 10.0 - Technical Knowledge } \\
\text { \& Skills: } 10.2,10.3 \text {, }\end{array}$ \\
\hline
\end{tabular}




\section{Agriculture Sales:}

\begin{tabular}{|l|l|}
\hline & Proper dress for the position. \\
\hline & Aunctual \\
\hline & $\begin{array}{l}\text { Pdhering to work schedule and flexibility during the holiday season. } \\
\text { phones. }\end{array}$ \\
\hline $\begin{array}{l}\text { Cashier skills: use of credit card machine, take checks, or take money and give } \\
\text { accurate change. }\end{array}$ \\
\hline Ability to accept constructive criticism. \\
\hline Ability to follow directions \\
\hline Computer Skills \\
\hline Good spelling- as it relates to spelling customer names and typing messages. \\
\hline $\begin{array}{l}\text { Wholesale versus retail markets: ability to identify the different types of } \\
\text { business }\end{array}$ \\
\hline Business types: sole proprietor, partnerships, corporations, and cooperatives. \\
\hline Sales Invoice: how to create and or complete. \\
\hline Business Cards: ability to prepare professional business cards. \\
\hline $\begin{array}{l}\text { Business Flyers: proper use of color, layout and appropriatc quantity of } \\
\text { information }\end{array}$ \\
\hline $\begin{array}{l}\text { Job Acquisition Skills: written application, resume, letter of application, and } \\
\text { references. }\end{array}$ \\
\hline Job Interview Skills- professional dress, interview questions, and follow up. \\
\hline Agriculture Sales- Knowledge of the 7 sales steps. \\
\hline Features and Benefits Presentation of products and or services. \\
\hline SWOT Analysis. (Strengths, Weaknesses, Opportunities, Strengths) \\
\hline Ability to develop a Marketing Proposal \\
\hline $\begin{array}{l}\text { Ability to uəc rc3ourcc3 including, but not limitcd to, the intcrnct, Rcacarch } \\
\text { Journals, and professional contracts to find appropriate information. }\end{array}$ \\
\hline
\end{tabular}


Veterinary Science:

\begin{tabular}{|l|l|}
\hline & Proper dress for the position. \\
\hline & Punctual \\
\hline & Adhering to work schedule and flexibility during the holiday season. \\
\hline Ability to accept constructive criticism. \\
\hline Ability to follow directions \\
\hline $\begin{array}{l}\text { Identification of major livestock breeds: horses, cattle (dairy \& beef), swine, } \\
\text { sheep and goats }\end{array}$ \\
\hline $\begin{array}{l}\text { Common livestock diseases and parasites: prevention, identification and } \\
\text { treatment. }\end{array}$ \\
\hline Internal and external anatomy of livestock \\
\hline Physiology of body systems of all livestock. \\
\hline Dcmonotratc jafc handling of livçtock. \\
\hline Identify wholesale and retail cuts of meat. \\
\hline Identify signs of estrus and approaching parturition. \\
\hline Detcrminc drc3aing pcrccntagc. \\
\hline Calculate average daily gain, the cost per pound of feed, feed efficiency, etc. \\
\hline $\begin{array}{l}\text { Identify common livestock tools and use them: vaccination syringe, dehorner, } \\
\text { burdizzo, hoof trimmcr, dosc oyringc, branding, tattooing, car tagging } \\
\text { equipment. }\end{array}$ \\
\hline Identify visual symptoms of a sick animal \\
\hline Rccognizc dystocia problcms \\
\hline Demonstrate live animal evaluation and provide reasons/justification \\
\hline Ability to read a market report \\
\hline Ability to read a futures market report. \\
\hline
\end{tabular}




\section{Supporting Completion \\ Materials S: Teaching Credentials}

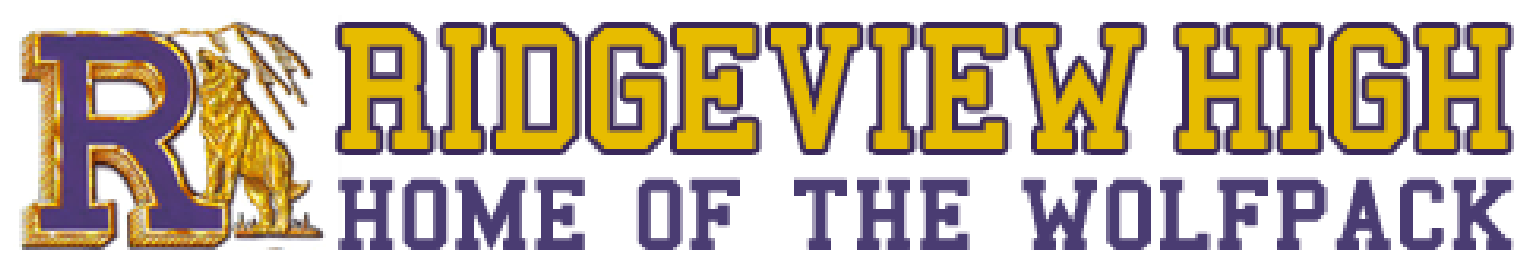




\section{S. Teaching Credentials}

Supporting Completion Materials Attached 


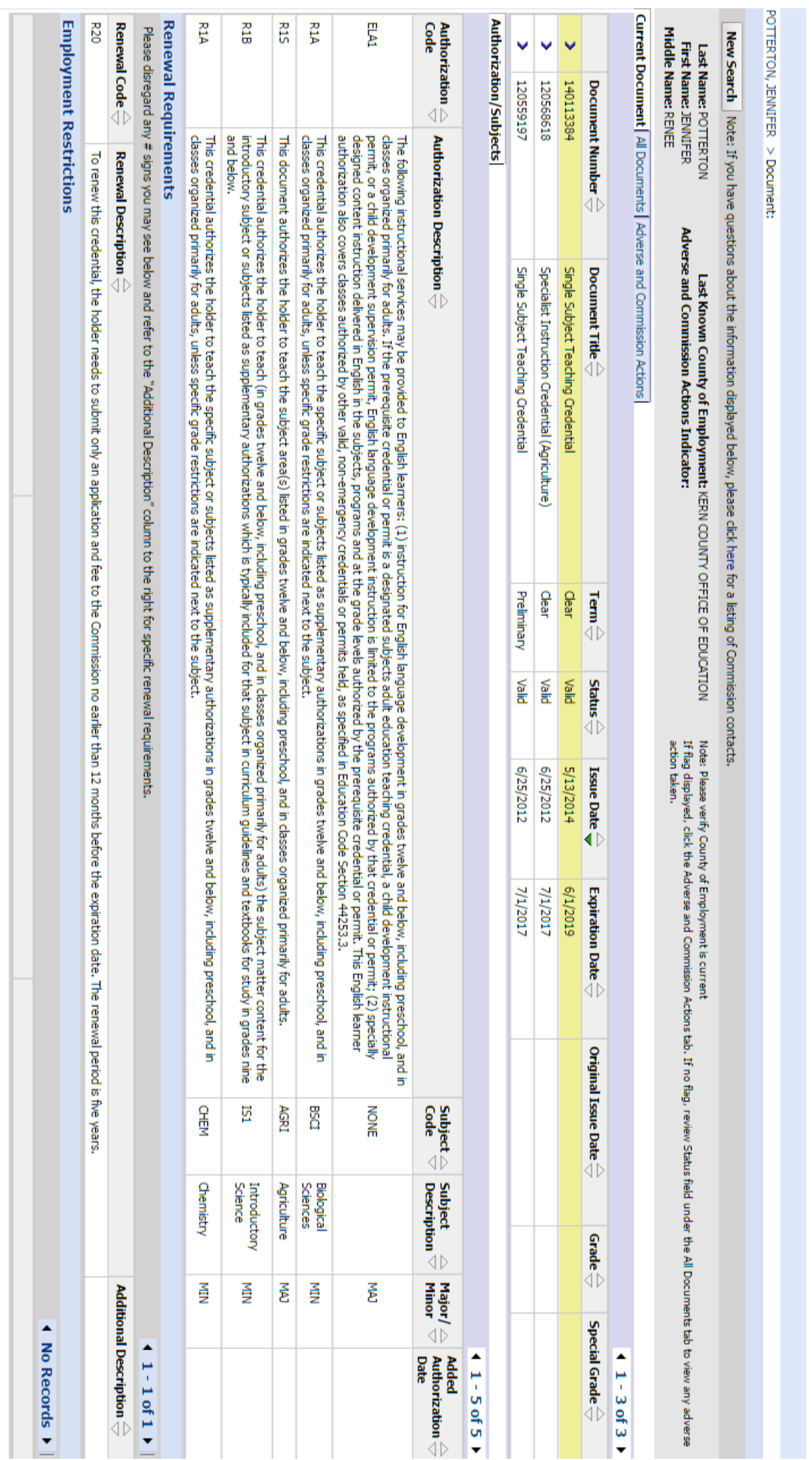


Supporting Completion Materials T: Department Calendar

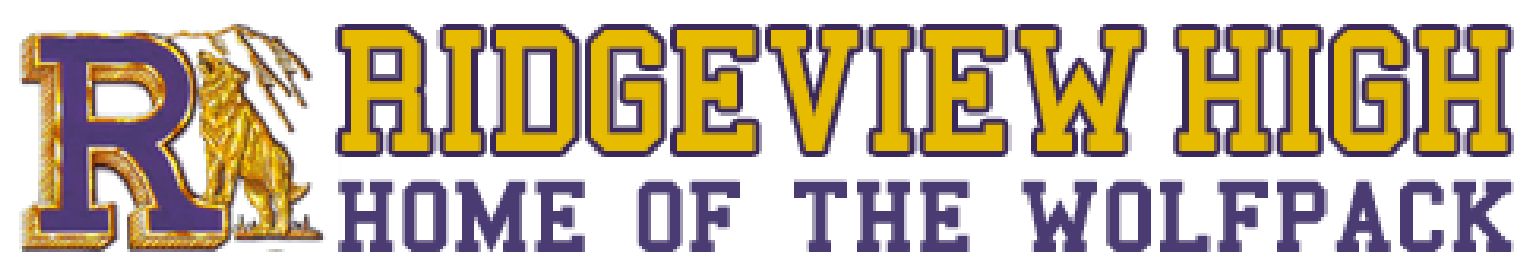




\section{T. Department Calendar}

Starting this year, the department calendar will be kept as a google calendar and will be linked on my website so the students constantly have access to it.

Supporting Completion Materials Attached 


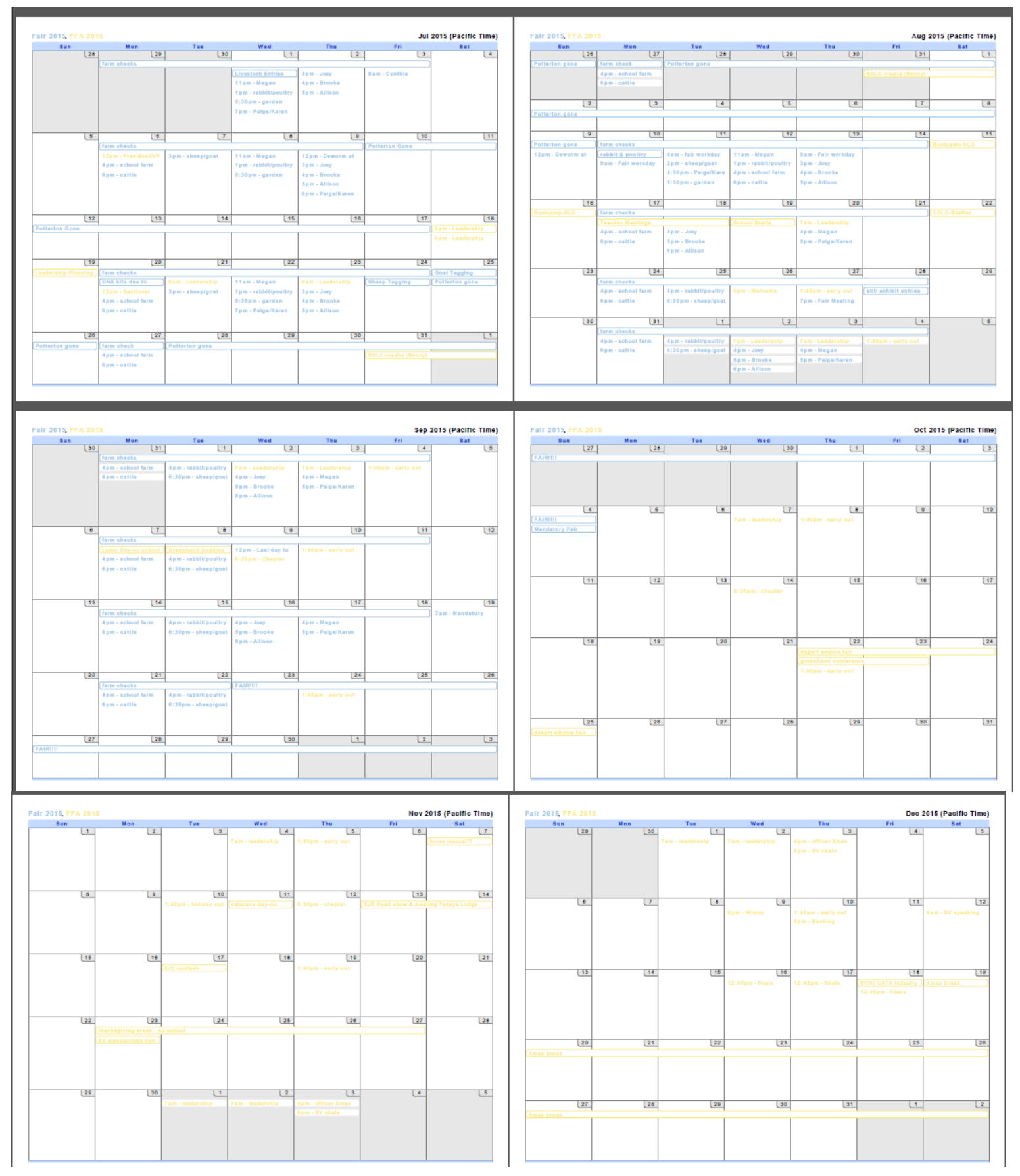



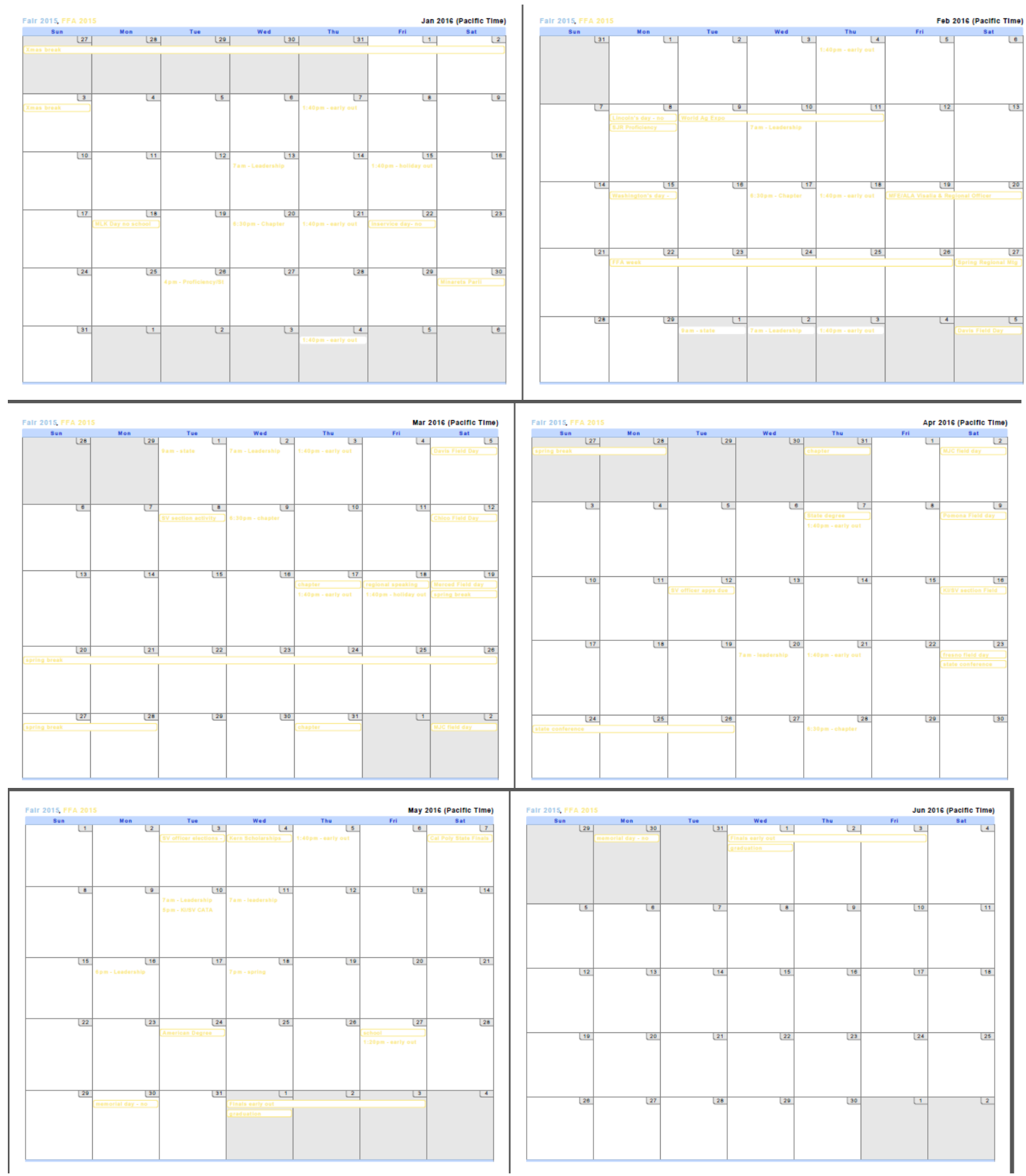


\section{Supporting Completion Materials U: Daily Logs}

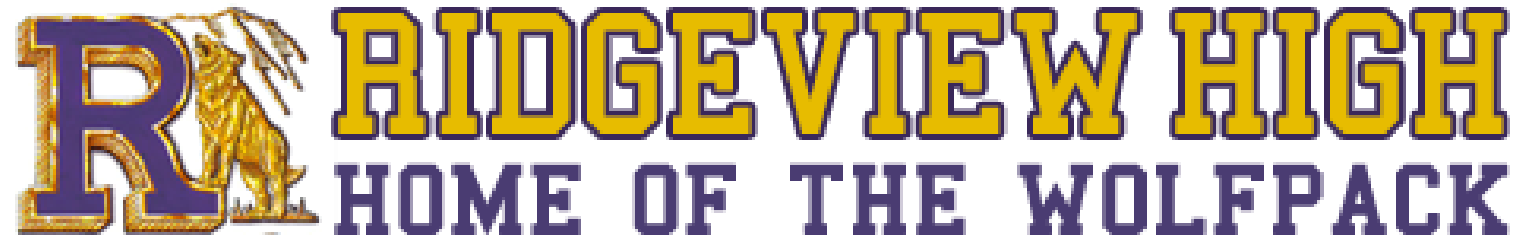




\section{U. Daily Logs}

Supporting Completion Material Attached 


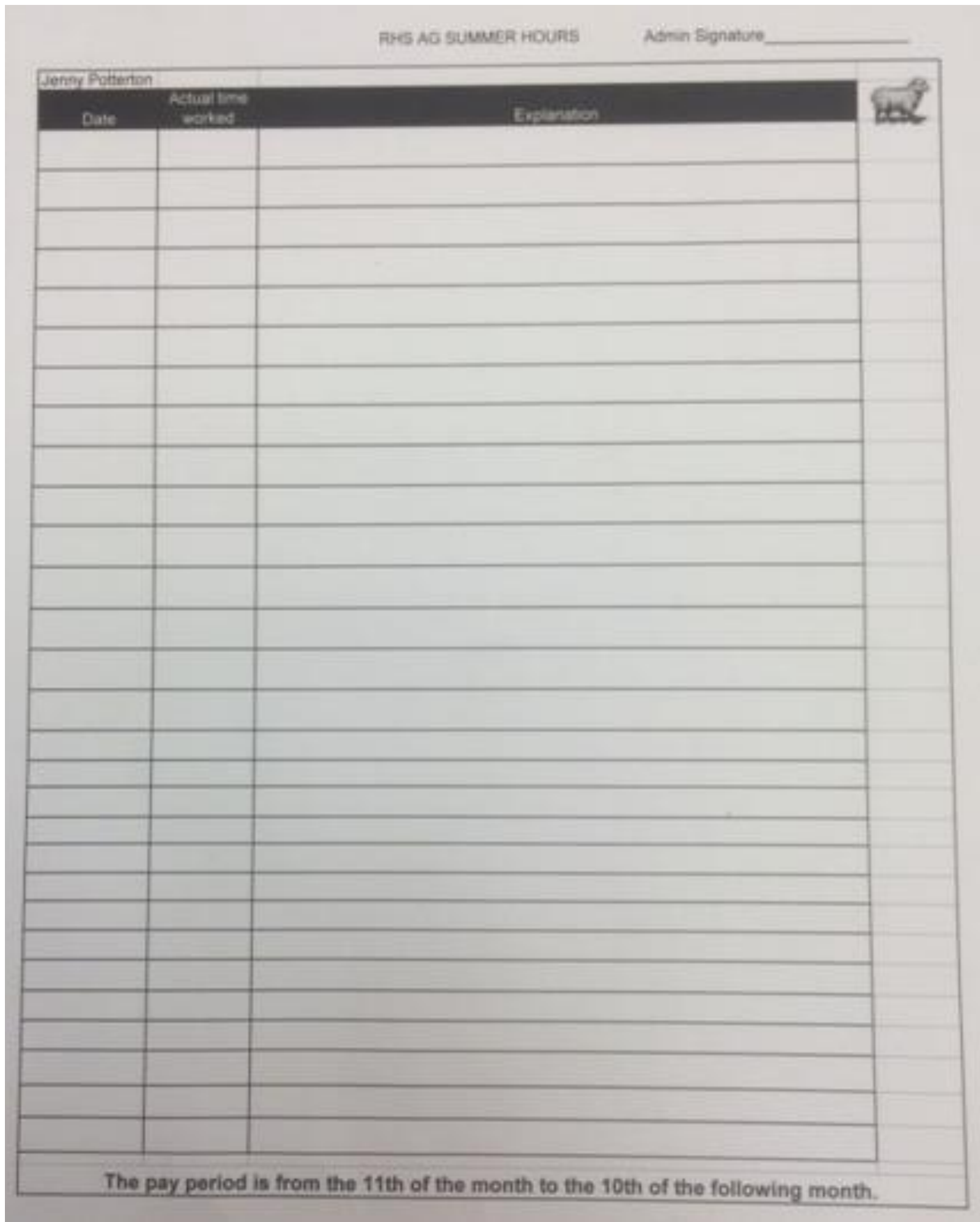


Supporting Completion

Materials V: Professional Growth Activities

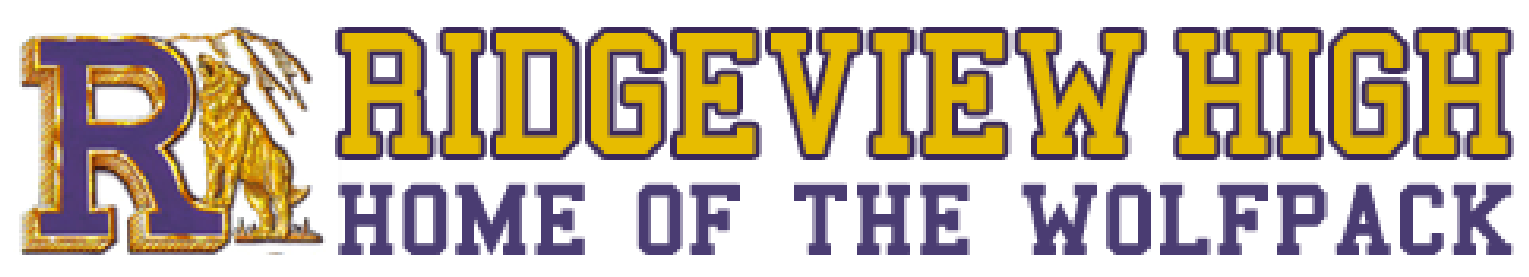




\section{Professional Growth Activities}

Expected Professional Growth Activities for 2015-2016

June 2015: CATA Summer Conference

August 2015: Sectional CATA Inservice Meeting

October 2015: Sectional CATA Inservice Meeting

November 2015: Regional CATA Meetings \& Roadshow

December 2015: Sectional CATA Inservice Meeting

January 2016: Sectional CATA Inservice Meeting

February 2016: Regional CATA Meetings

May 2016: Sectional CATA Inservice Meeting 


\section{Supporting Completion Materials W: R2 Report}

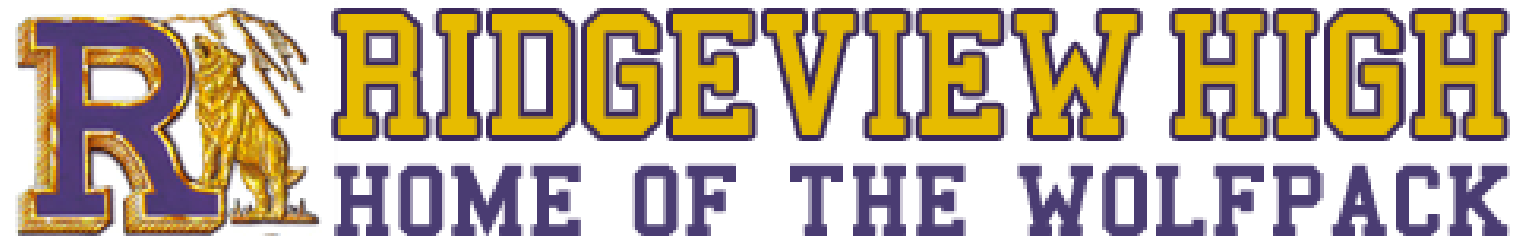




\section{W. R2 Report}

Supporting Completion Material Attached 
Ridgeview HS

R2 Student Report

Year:2014

Gender

\begin{tabular}{|l|l|l|l|}
\hline Schnum & ProgName & Male & Female \\
\hline 352 & Ag Bus Mgt & 0 & 1 \\
\hline 352 & Agriscience & 57 & 108 \\
\hline 352 & An. Science & 0 & 1 \\
\hline
\end{tabular}

Hispanic

\begin{tabular}{|l|l|l|}
\hline ProgName & Hispanic & Non-Hispanic \\
\hline
\end{tabular}

\begin{tabular}{|l|l|l|}
\hline Ag Bus Mgt & 1 & 0 \\
\hline Agriscience & 98 & 67 \\
\hline An. Science & 0 & 1 \\
\hline
\end{tabular}

Race*

\begin{tabular}{|l|l|l|l|l|l|l|l|}
\hline ProgName & White & Black & Hispanic & Americian Indian & Asian & Native Hawaiian/Pacifc Island & 2 or more \\
\hline Ag Bus Mgt & 1 & 0 & 0 & 0 & 0 & 0 & 0 \\
\hline Agriscience & 134 & 7 & 0 & 6 & 6 & 4 & 12 \\
\hline An. Science & 1 & 0 & 0 & 0 & 0 & 0 & 0 \\
\hline
\end{tabular}

Grade Level

\begin{tabular}{|l|r|r|r|r|r|r|r|r|r|}
\hline Year In Ag & Grade9 & Grade10 & Grade11 & Grade12 & Grade13 & Grade14 & Grade15 & Grade16 & Total \\
\hline 1 & 97 & 0 & 2 & 0 & 0 & 0 & 0 & 0 & 99 \\
\hline 2 & 0 & 30 & 3 & 2 & 0 & 0 & 0 & 0 & 35 \\
\hline 3 & 0 & 0 & 19 & 0 & 0 & 0 & 0 & 0 & 19 \\
\hline 4 & 0 & 0 & 0 & 13 & 0 & 0 & 0 & 0 & 13 \\
\hline 6 & 0 & 0 & 0 & 0 & 0 & 1 & 0 & 0 & 1 \\
\hline Total & 97 & 30 & 24 & 15 & 0 & 1 & 0 & 0 & 167 \\
\hline \multicolumn{9}{|c|}{} & \multicolumn{10}{|c|}{ Total 9-12 } & 166 \\
\hline
\end{tabular}

Freshman Persistance:

Cohort Year: 2011-2012

\begin{tabular}{|l|r|r|}
\hline Years in Ag Completed & Count & Percent \\
\hline 1 & 71 & $62 \%$ \\
\hline 2 & 27 & $23 \%$ \\
\hline 3 & 4 & $3 \%$ \\
\hline 4 & 13 & $11 \%$ \\
\hline Freshman Cohort Students & 115 & \multicolumn{1}{|l}{} \\
\cline { 1 - 2 } Average Years Completed & 1.6 & \multicolumn{2}{|l}{} \\
\cline { 1 - 2 }
\end{tabular}

*Prior to 2010 Hispanic is listed as a race.

Printed: 7/2/2015 12:22:06 PM 


\section{Detail FFA Roster}

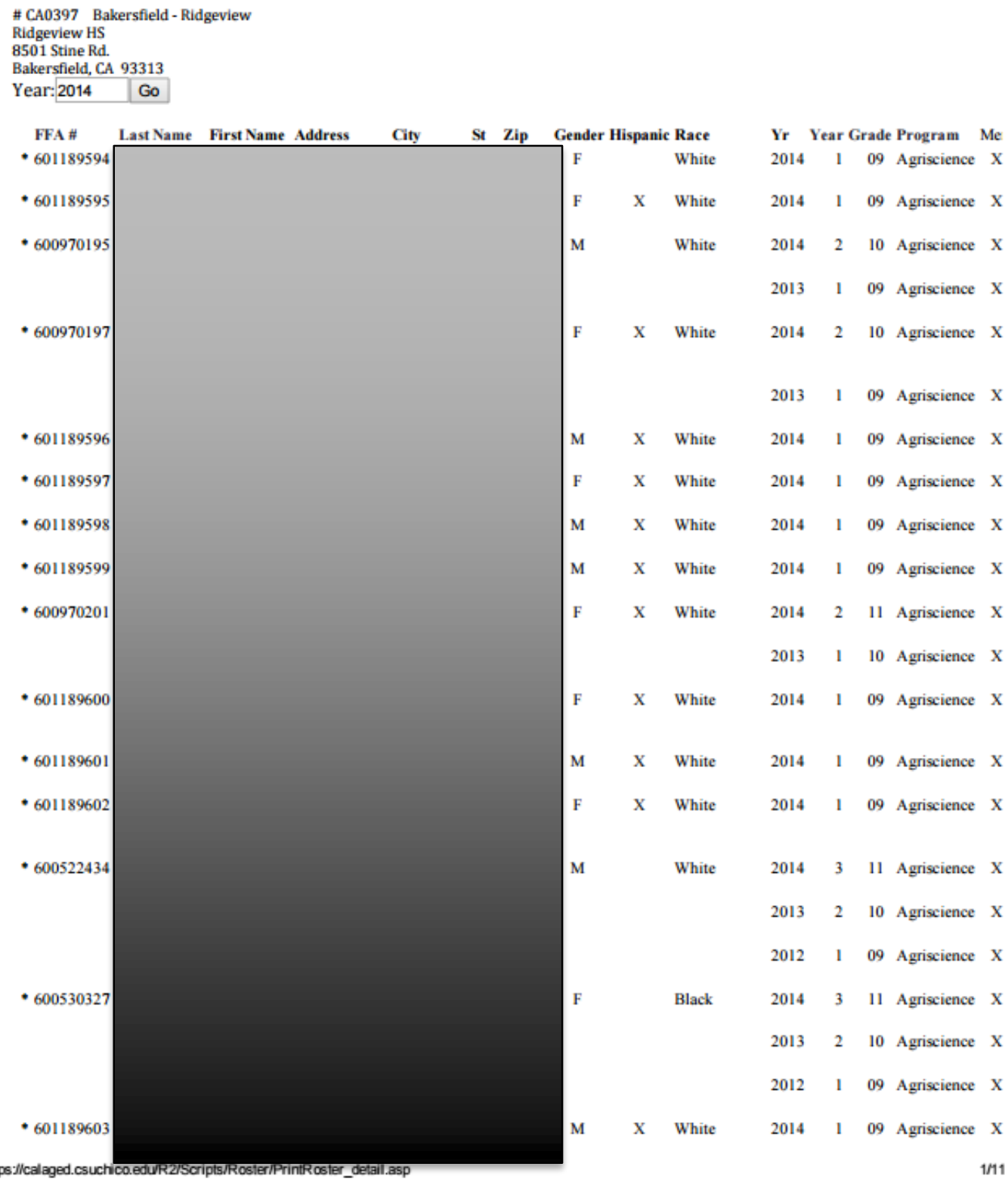




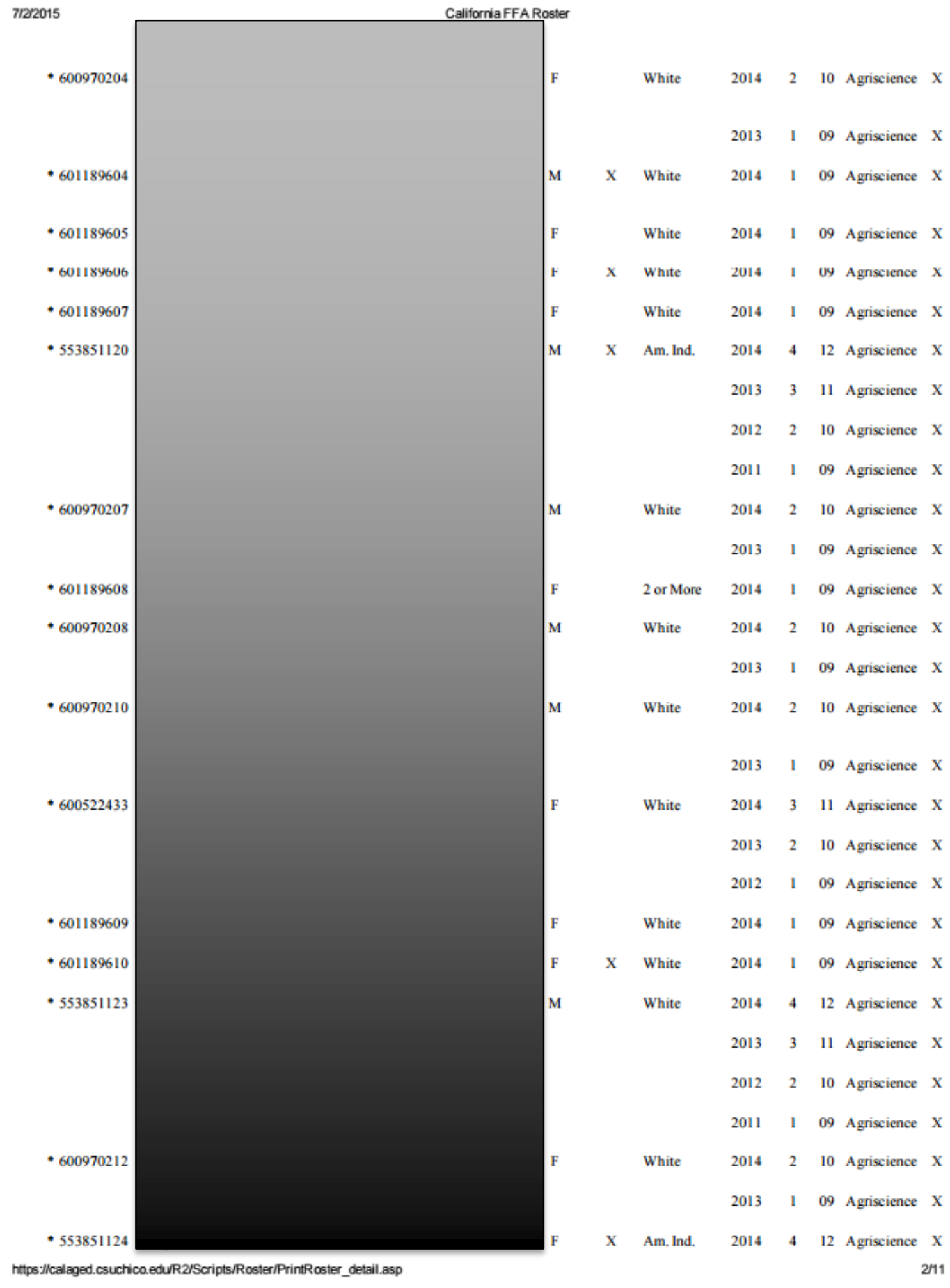




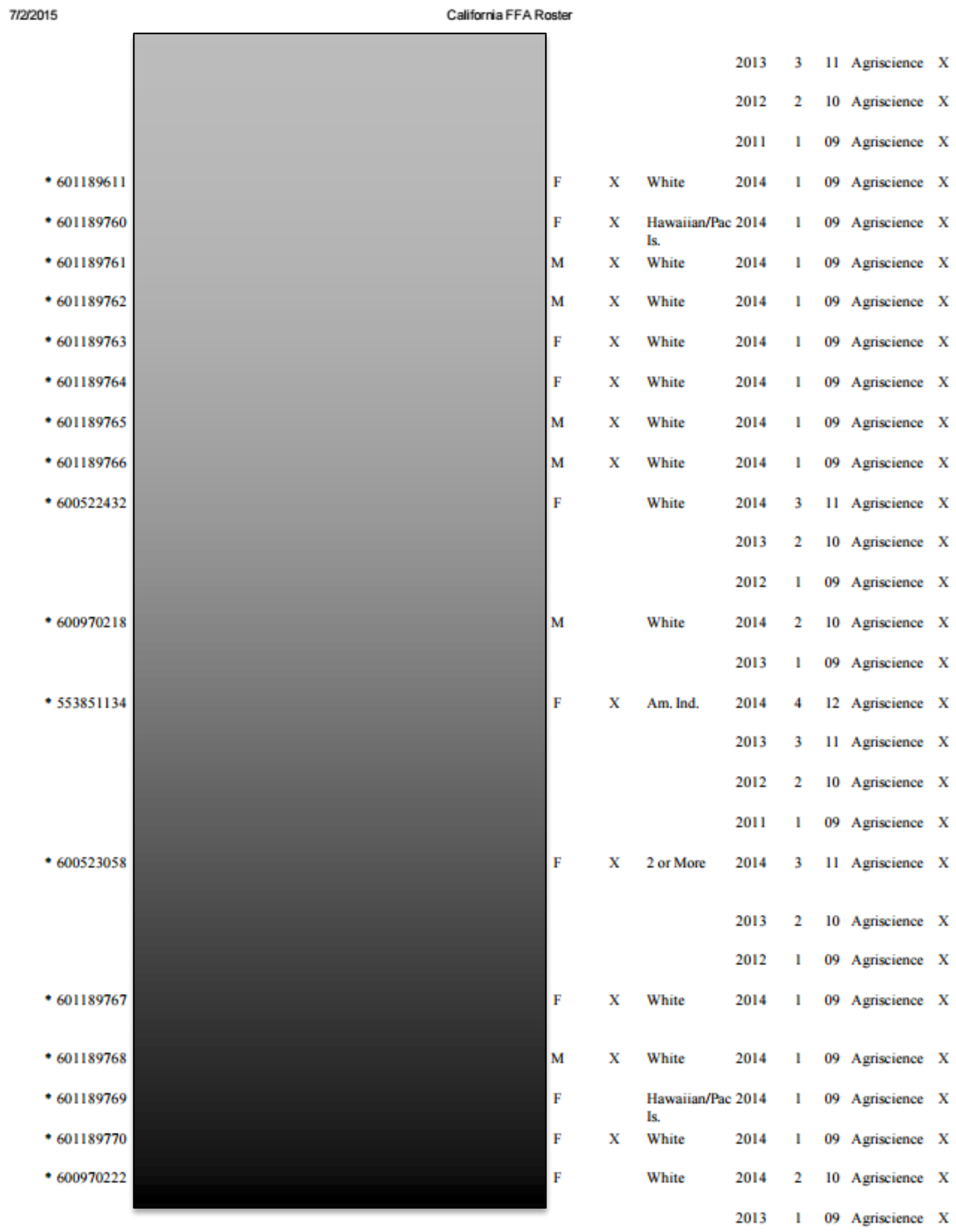

https:/licalaged.csuchico.edu/R2/Scripts/Roster/PrintRoster_detail.asp 

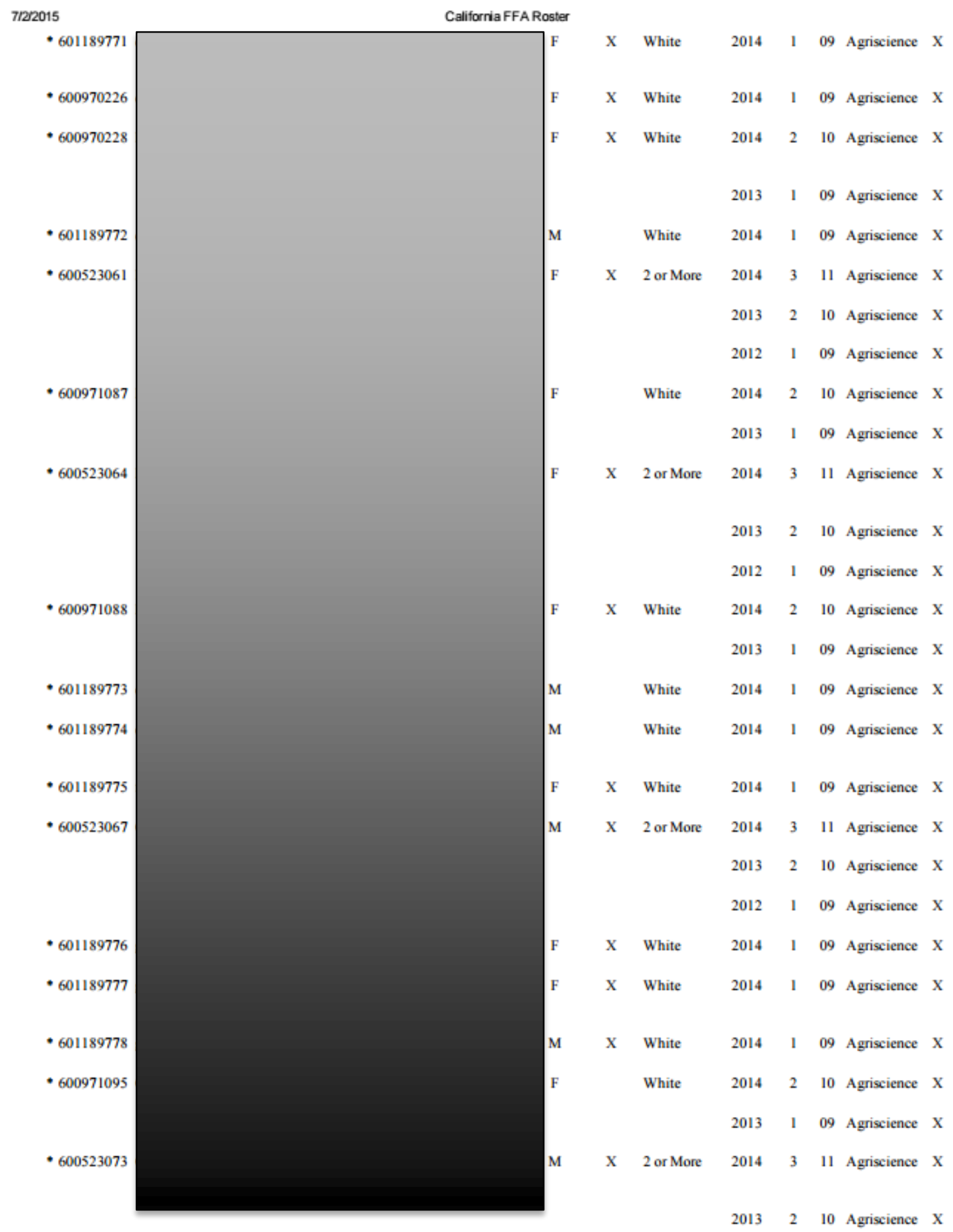

https://calaged.csuchico.edu/R2/Scripts/Roster/PrintRoster_detail.asp 


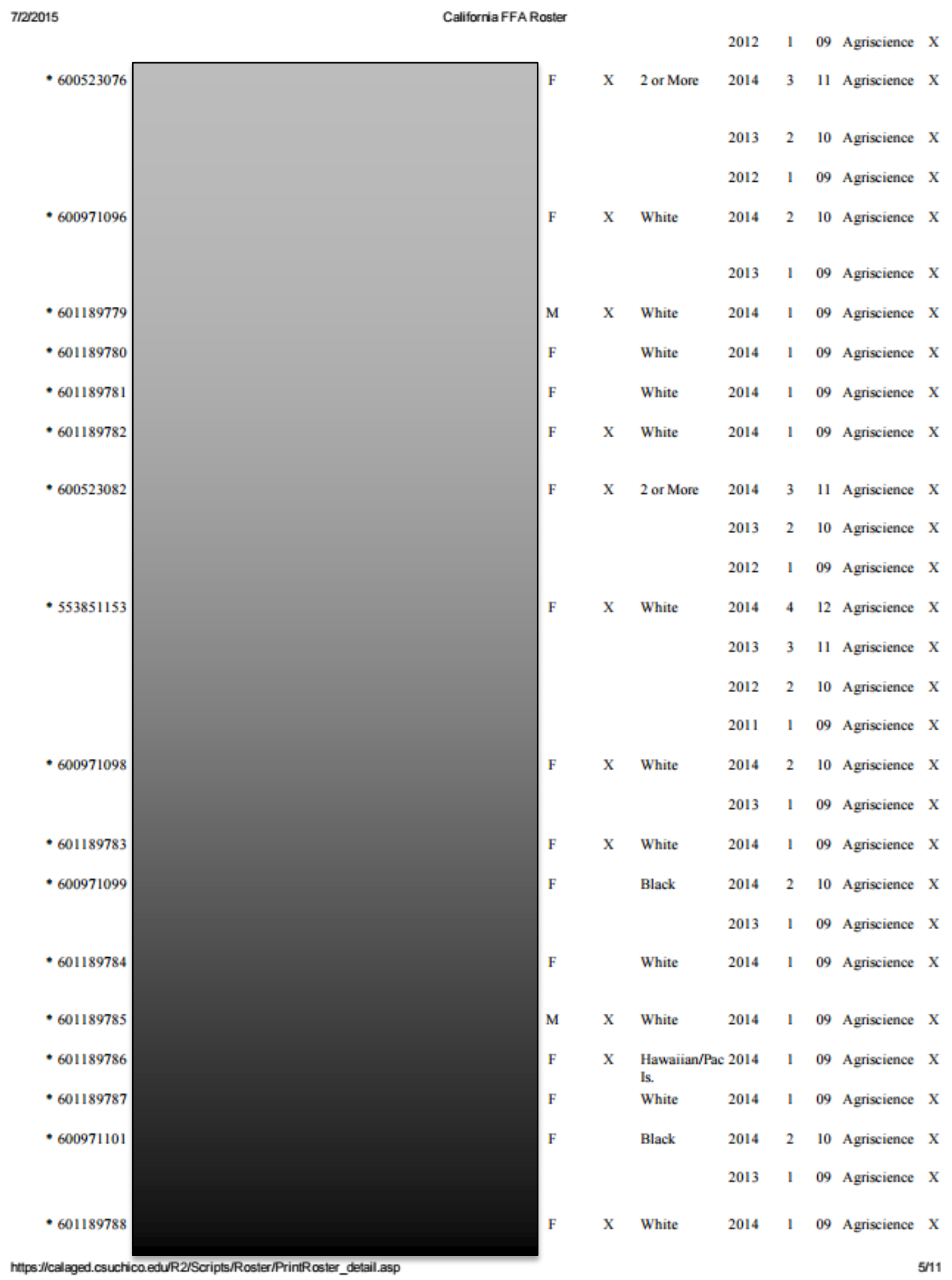




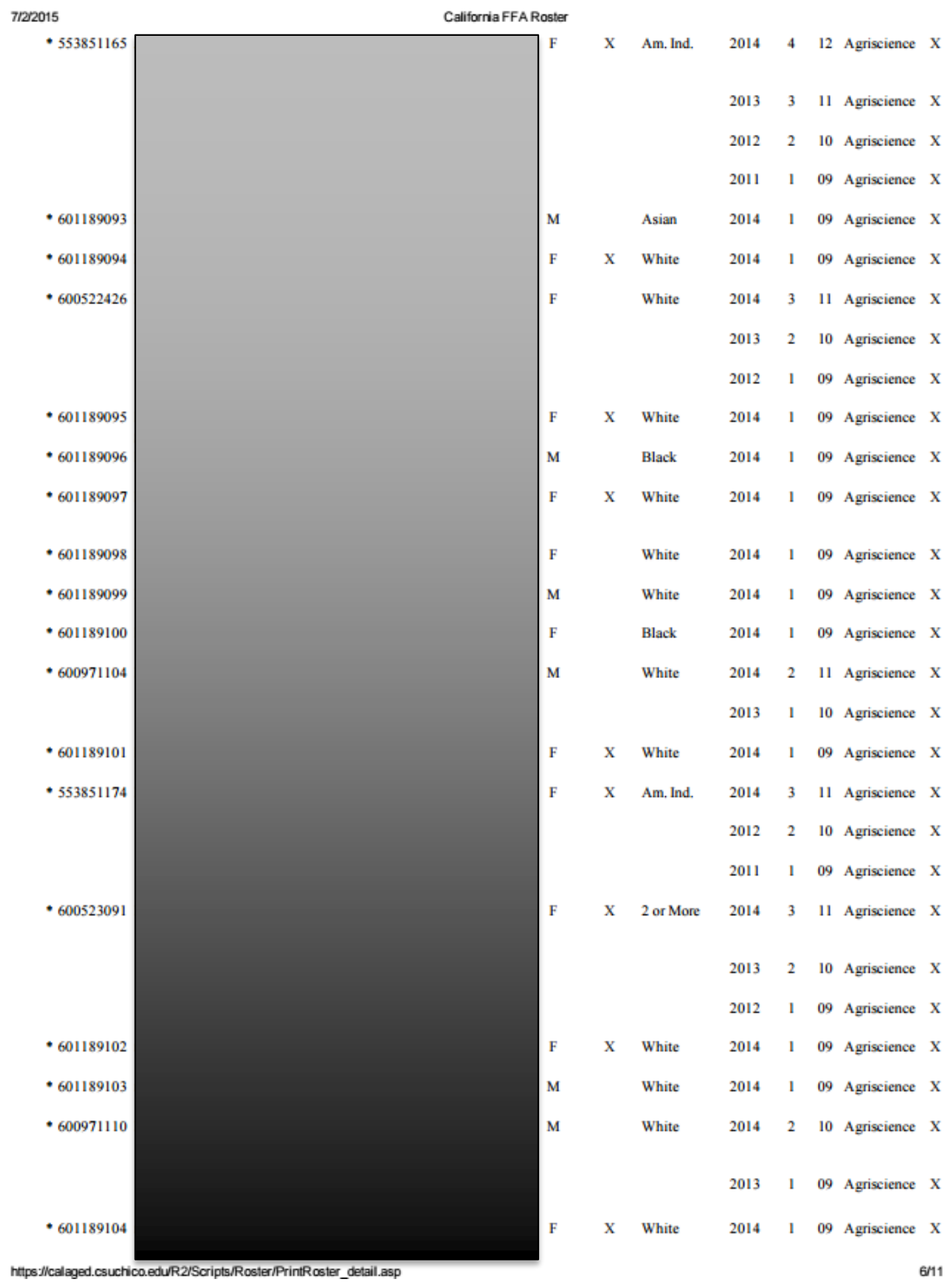




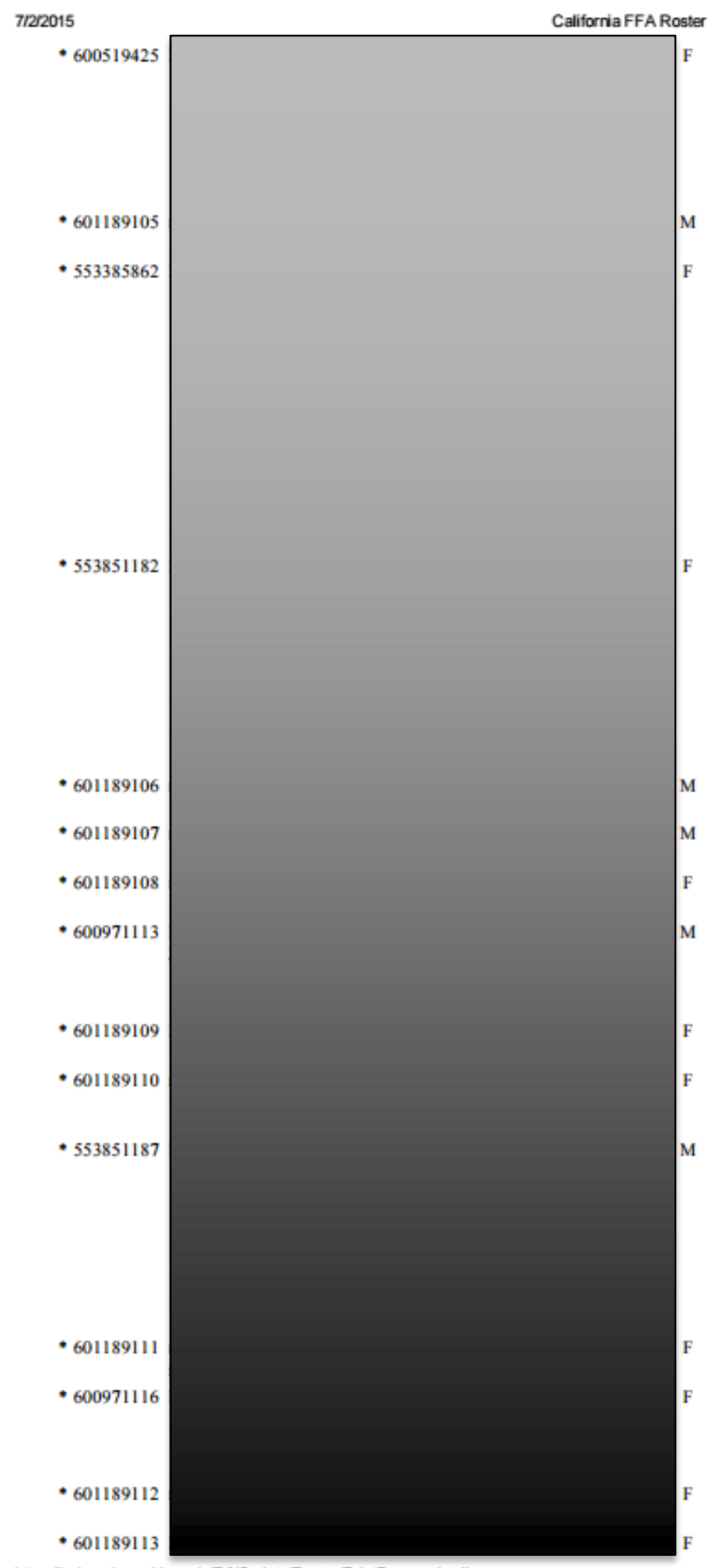

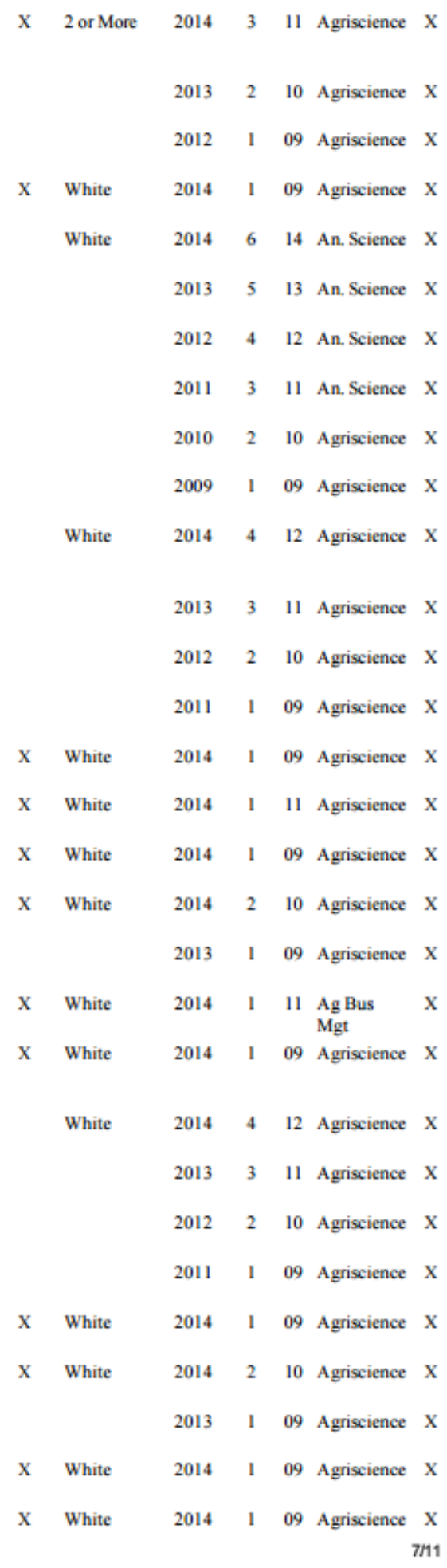




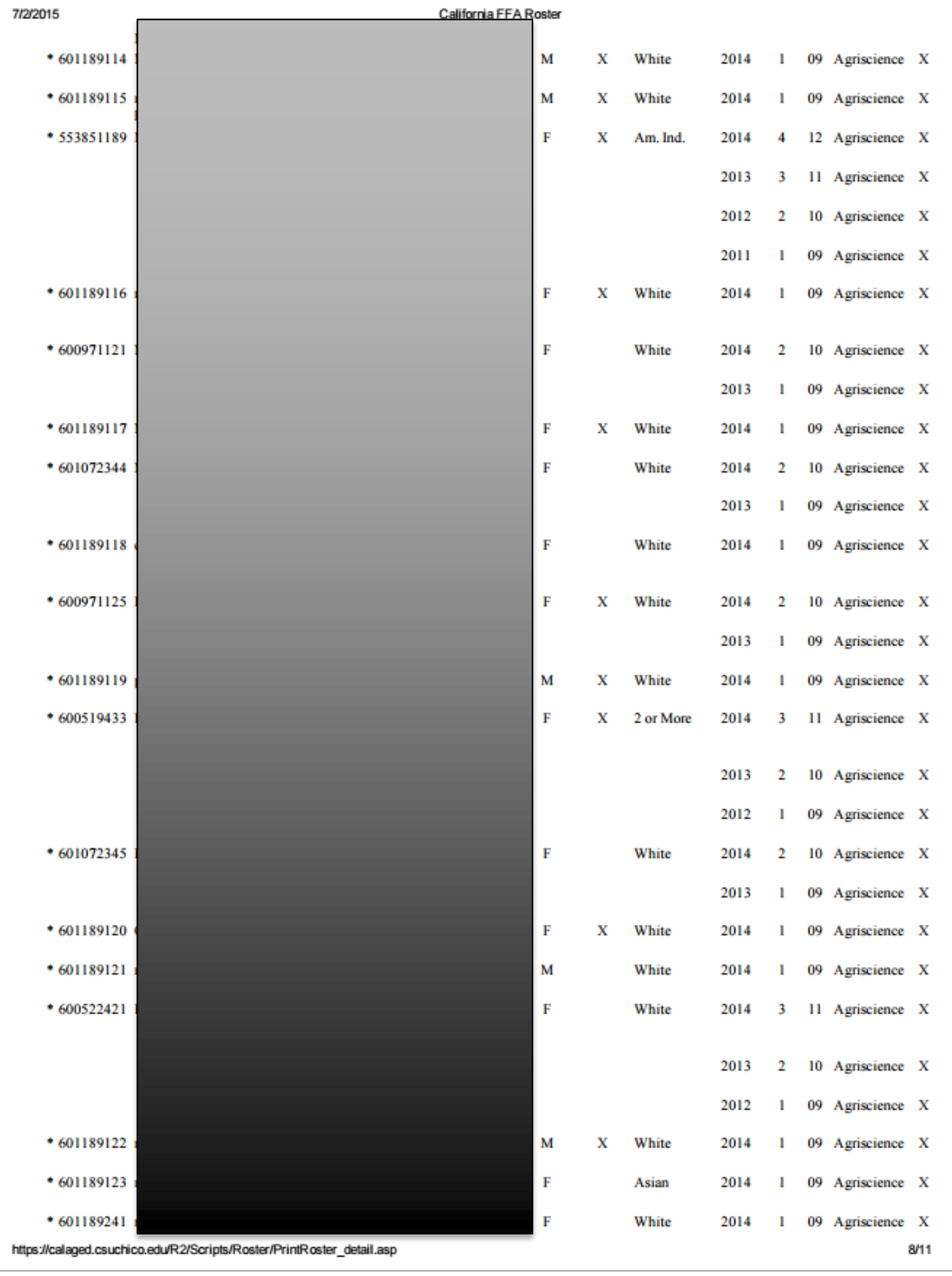




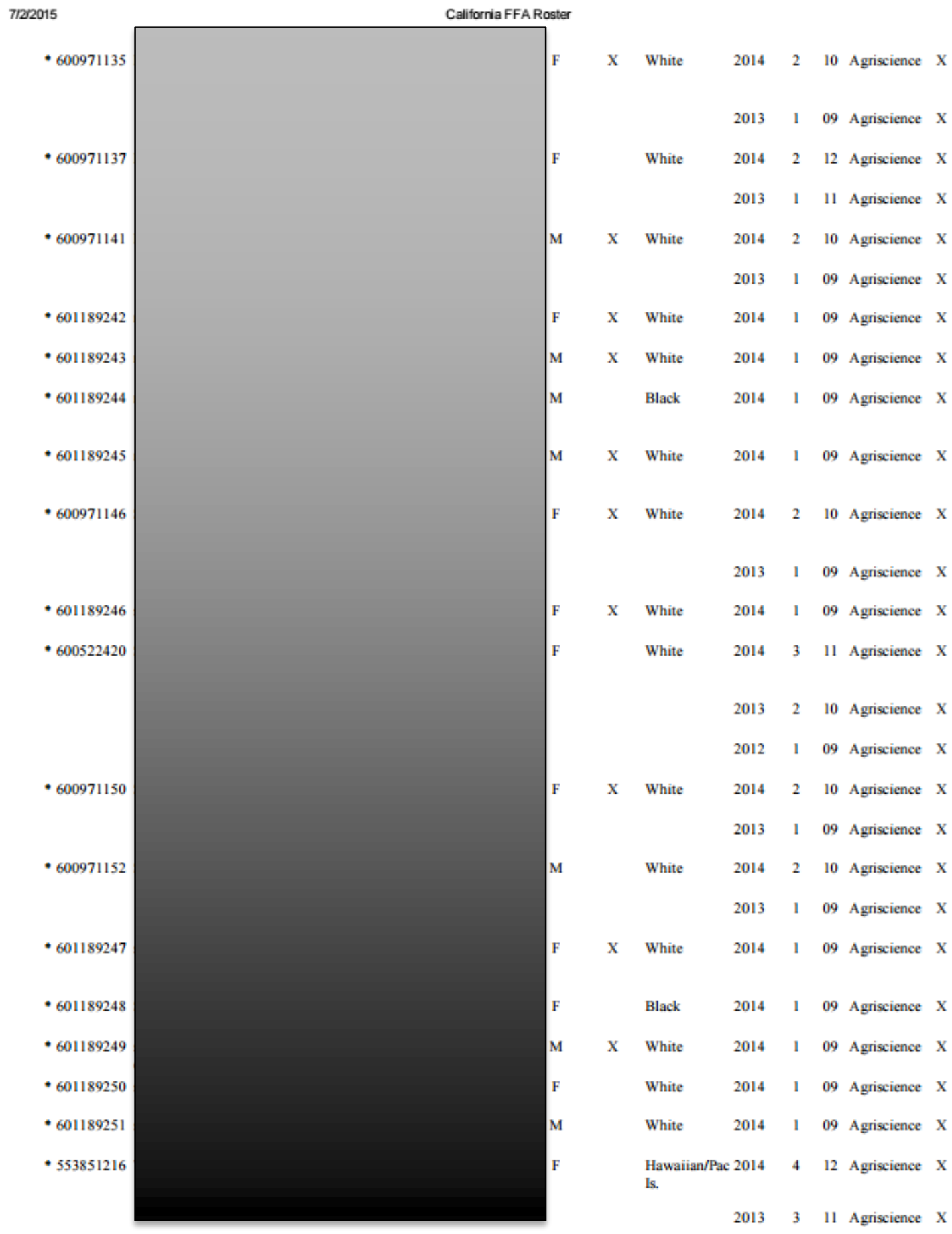

https:/lcalaged.csuchico.edu/R2/Scripts/Roster/PrintRoster_detail.asp

$9 / 11$ 


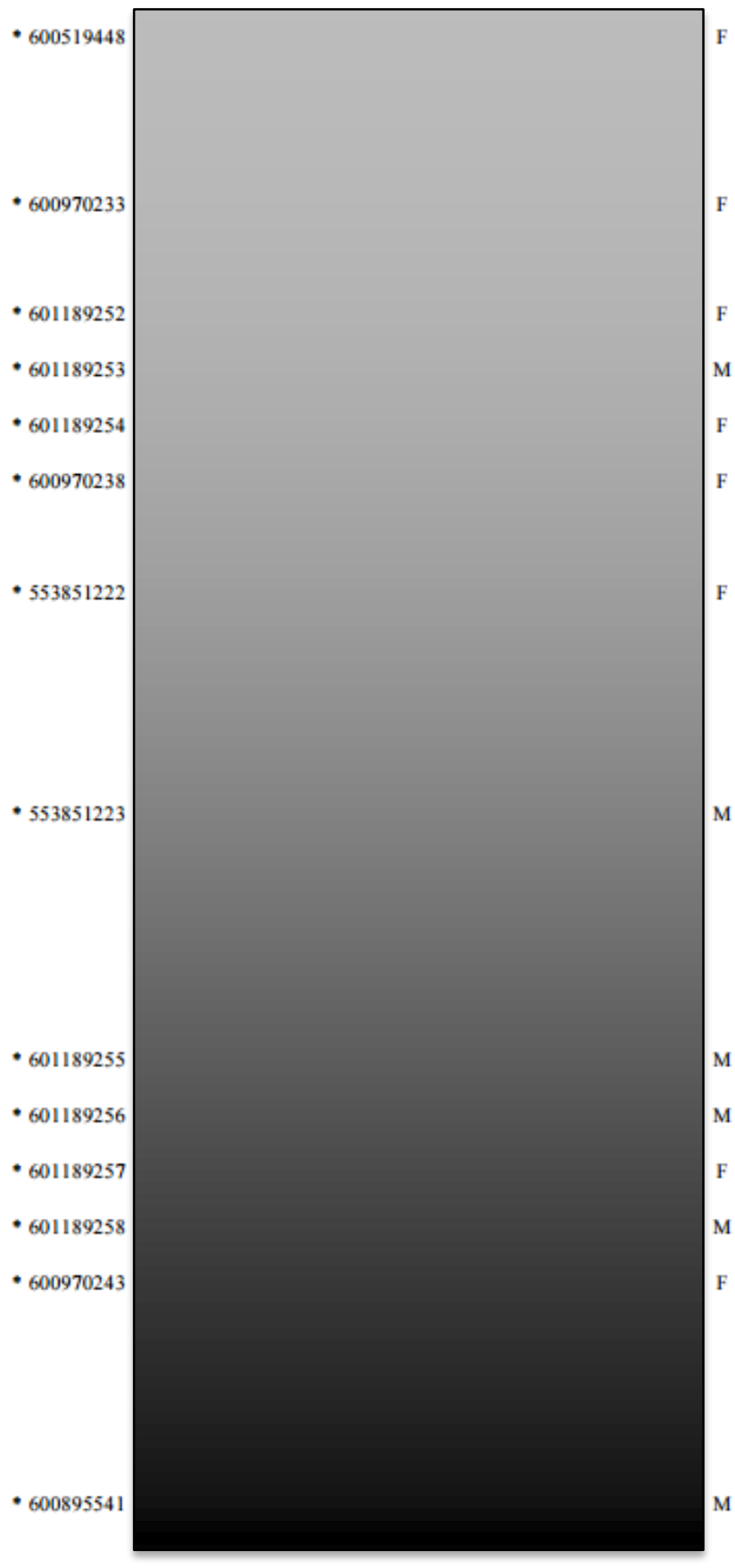

https:/lcalaged.csuchico.edu/R2/Scripts/Roster/PrintRoster_detail.asp

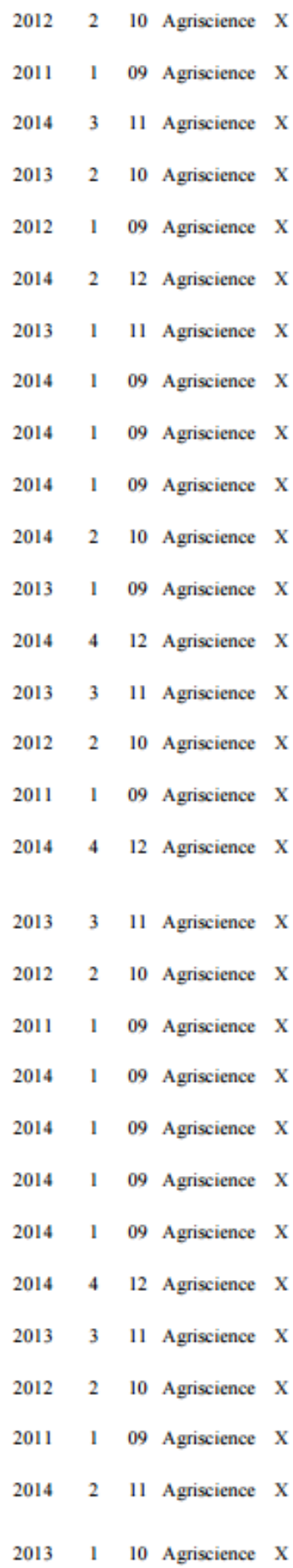




7/22015
$\cdot 601189259$
$\cdot 601189260$

Printed: 7/2/2015 12:23:40 PM

Count: 167

- Postad

Site developed and maintained by the California FFA Association. Page last modified: $3 / 20 / 2012$ 
Supporting Completion

Materials X: Extended Contract Rationale

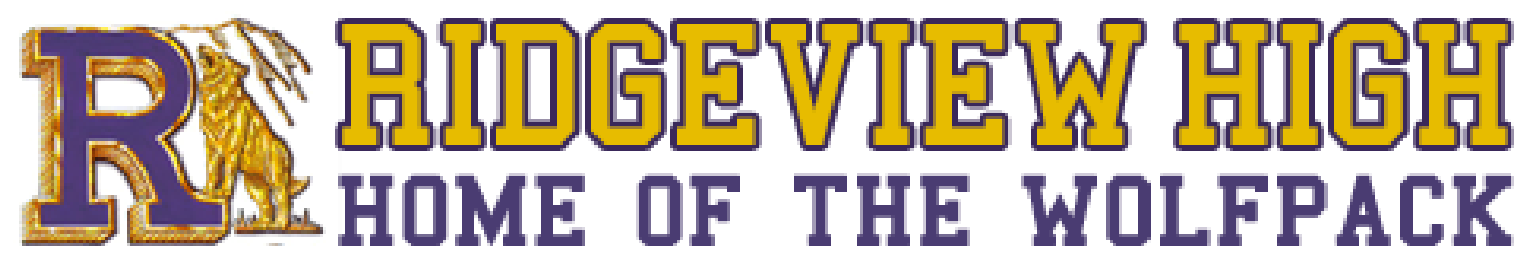




\section{Extended Contract Rationale}

Per our District Ag Coordinator, Mr. Ralph Mendes, there is no extended contract rationale or justification on file. The district provides summer pay to agriculture teachers in the district that is reflective of their agriculture course load. The

maximum days eligible for summer pay is 36 days at your regular salary rate. Any teacher that is not full time receives the percentage of 36 days equivalent to their agriculture coarse load. Throughout my employment in the district, there has been attempts to have the justification for pay written down. At this point, a few teachers in the district have been asked to sign paperwork related to their summer pay, but I have not been asked. 
Supporting Completion Materials Y: Completed Travel Plan

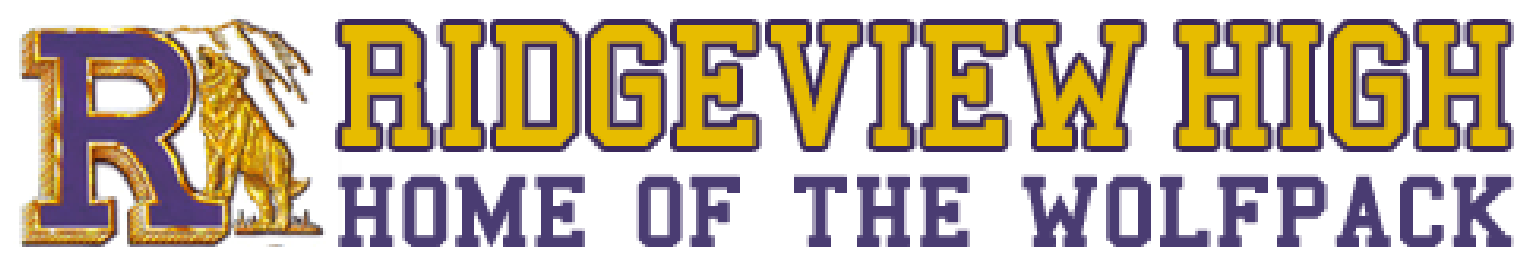




\section{Y. Completed Travel Plan}

The following pages are examples of field trip requests for both local and overnight trips. For out of state trips, a board action is also required. As each individual trip must have its own separate document, a small sampling has been provided in this section to show local and overnight trips. Overnight trips also require an itinerary. The field trip requests are signed by our vice principal who sends them to the board and a copy is sent to our Athletic secretary who reserves all vehicles.

Supporting Completion Material Attached 


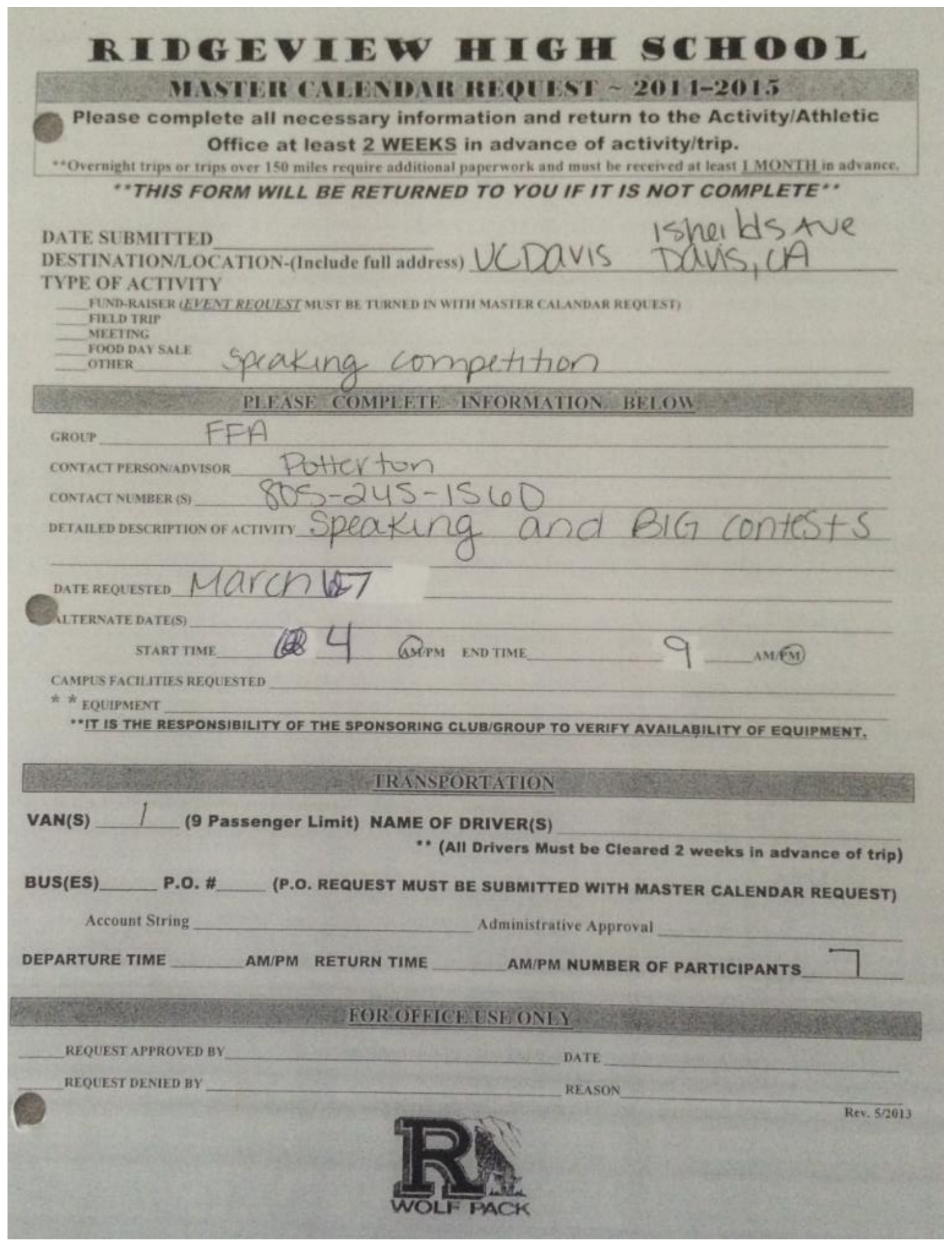




\section{OFFICE OF INSTRUCTION}

REUEST FOR APPROVAL OF FIELD TRIP, OUT OF AREA TRIP. AND/OR OVERNIGHT TRIP
Local Field Trip or Co-Curricular Trip
(School Site Administrative approval)
- Submit this from to local school administration at
least ten (10) days in advance of trip
X Out of Area Trip over 150 miles or overnight
(Assoc. Supt/Director of Insturction approval)
- complete from and return at least thirty $\left(30^{* *}\right)$ school days in advance of trip
Trip Out of State
(incl. Mexico and/or longer than 3 school days)
-Complete form and return at least sixty days in advance of trip
Trip to foreign country (except Mexico)
(Assoc. Supt/Director of Insturction approval)
- Complete form and return at least one year in advance of trip

- See back of sheet for additional forms

* Trips earned in compttition are exempt from this 30 day limit, but should be turned in as soon as possible

(Please Complete All Questions)

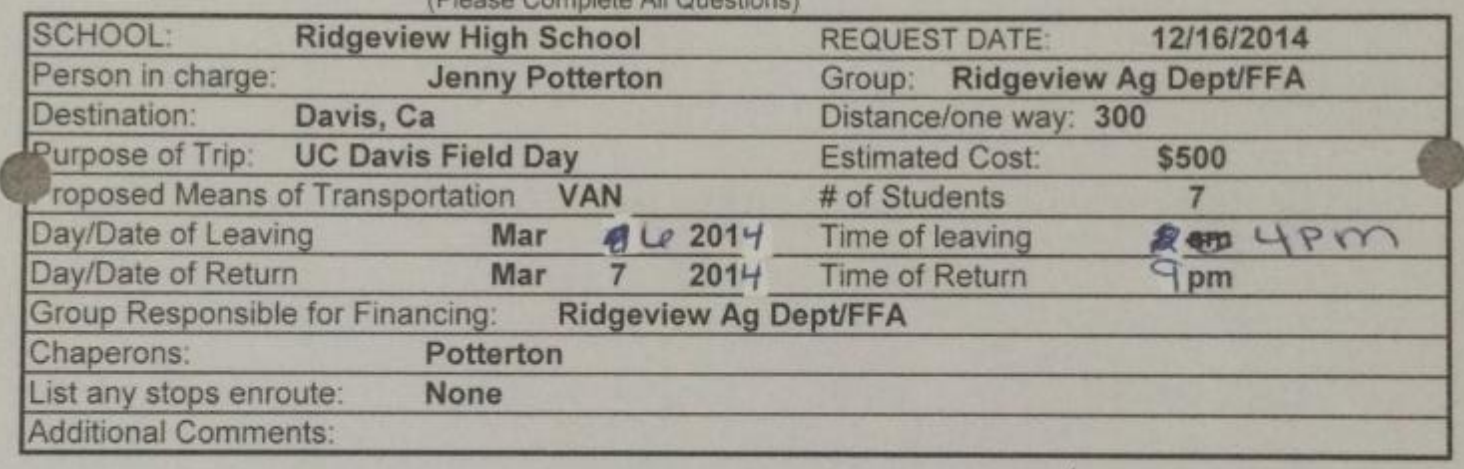

Approved:

\begin{tabular}{l}
\hline Director of Activites/Transportation \\
\hline Principal/Assistant Principal \\
\hline Director/School Support Services, Athletics (if athletics) \\
\hline Associate Superintendent and or Dirctor of Instruction \\
Superintendent/Secretary, Board of Trustees
\end{tabular}

\begin{tabular}{|c|}
\hline Date \\
\hline Date \\
\hline Date \\
\hline Date \\
\hline Date \\
\hline
\end{tabular}

(Submit in Duplicate of Office of Instruction) 
UC Davis Field Day Itinerary

Date: $3 / 6-7 / 15$

$3 / 6 / 15$

4:00 pm - Leave Ridgeview High School

9:00 am - Arrive Davis, CA

Hotel: University Park Inn and Suites

1111 Richards Boulevard, Davis, CA 95616

$10: 00 \mathrm{pm}$ - Lights out

$3 / 7 / 15$

7:30 am - Arrive UC Davis

1 Shields Avenue, Davis, CA 95616

7.45 am - Vegetable Crops contest begins at UC Davis

8:00 am - BIG and creed contests begin

3:00 pm $-1^{\text {st }}$ awards ceremony

$4: 30 \mathrm{pm}-2^{\text {nd }}$ awards ceremony

5:30 pm - Leave UC Davis

10:00 pm - Arrive RHS 


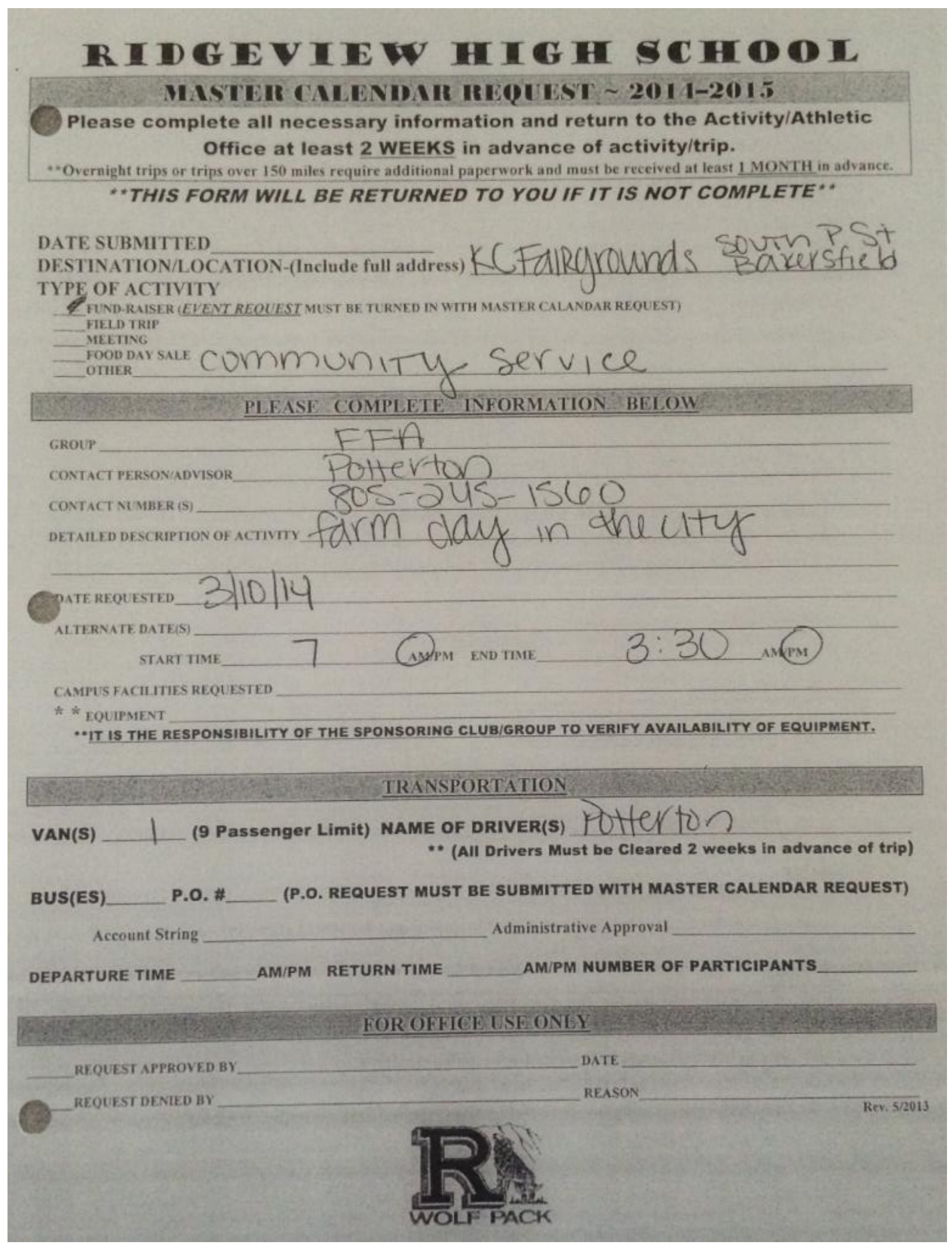




\section{KERN HIGH SCHOOL DISTRICT \\ REQUEST TO BE ABSENT \\ FROM ASSIGNED RESPONSIBILITIES FOR PROFESSIONAL ACTIVITIES}

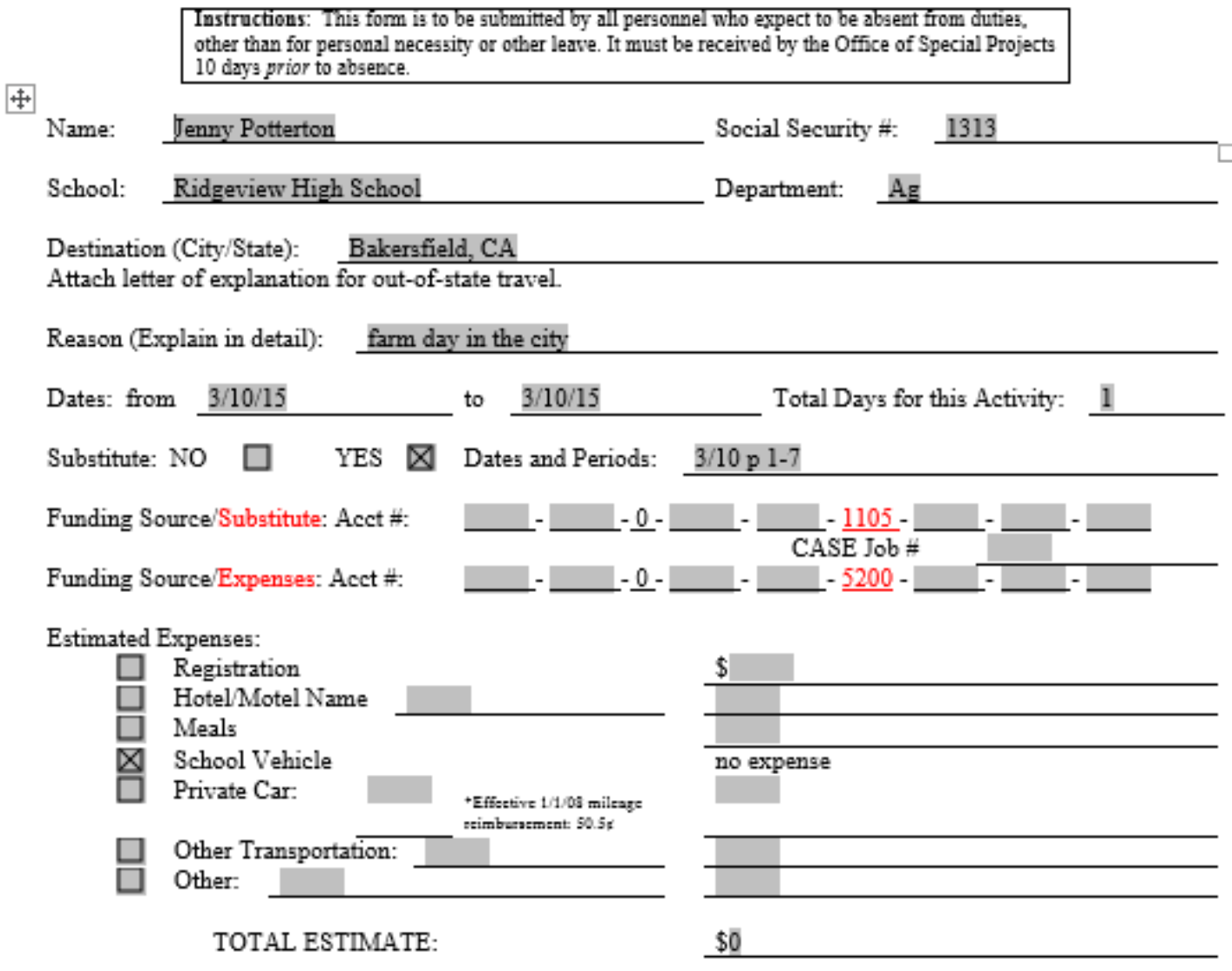

Date: Signature

SCHOOL AUTHORIZATION

The expenses listed above are approved.

Date:_ Principal's Signature:

DISTRICT AUTHORIZATION

Date:

District Approval:

(SEND TO OFFICE OF SPECIAL PROJECTS) 
Supporting Completion

Materials Z: CATA

Membership Card

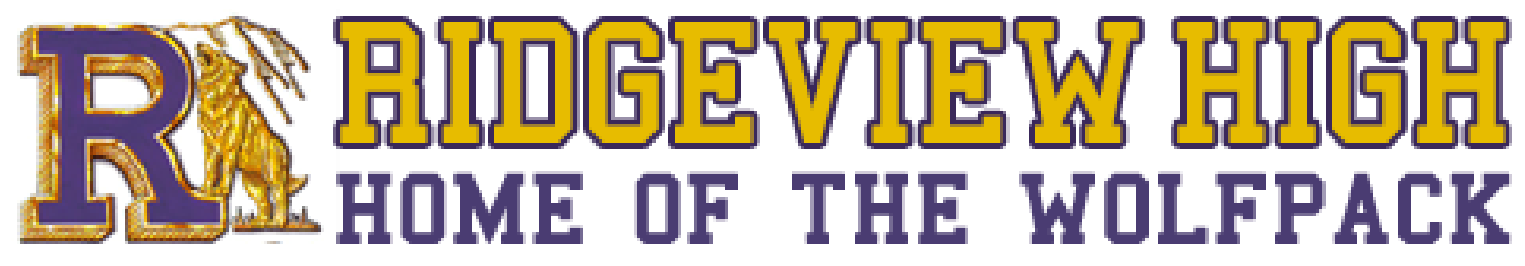


Z. CATA Membership Card

CALIFORNIA AGRICULTURAL TEACHERS' ASSOCIATION

Venny Potter ton 2015/2016 ACTIVE MEMBER 
Supporting Completion

Materials AA: Reports

Submitted to

Administration

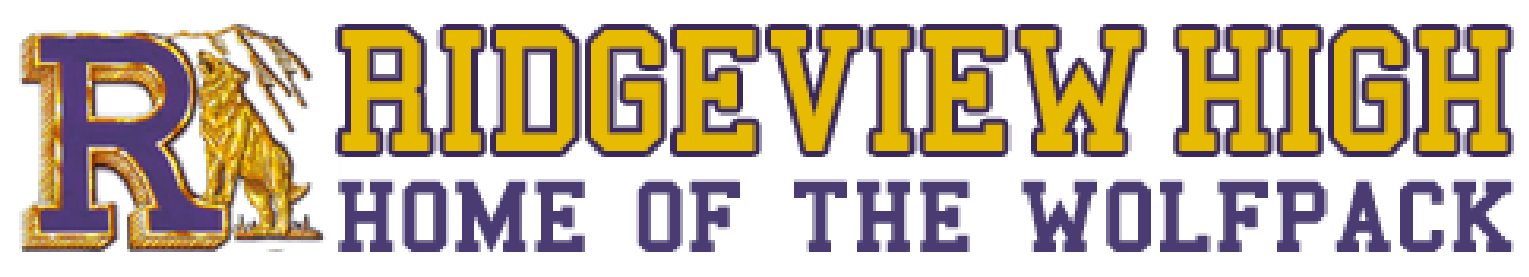




\section{AA. Reports Submitted to Administration}

Supporting Completion Material Attached 


\section{Ridgeview High School \\ Club Meeting MINUTES}

1. Name of Club Rigdeview FFA

2. The meeting was called to order by Connie contreras

3. Date of meeting $11 / 5 / 14 \quad$ Time $7: 00$ AM

4. The minutes of the previous meeting were:

Read and Approved Corrected and Approved

5. The treasurer reported $\$$ in our account.

6. Expenses incurred since last meeting:

7. The following purchases were approved:

Purpose/ltems Vendor Amount
A. Clipper Repair Jenny Potterton
$\$ 212^{43}$
B. Speaking contests Wasco FFA $\$ 120$ max
c. Poinsettias Foothill FFA
California FFA
D. MFE/ALA
Moved By: Jennifer España Seconded By: Kiani Tafulu
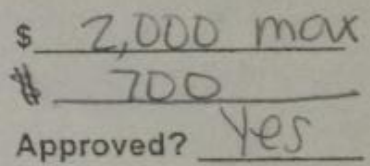

8. Communications and Reports:

9. Old Business: Last meeting we had a haurited hoube/garden 10. New Business: OlC Nov.18, Road Hoube Grill Dec.3, Public Speaking Leadership de Christmas Dinner Dec.4, Winter Banquet Dec. 13 , Skate Night Dec. 4 Nov. 12 Chapter Meeting Dot luck. 11. Those in Attendance (or see sign-in sheet):

On Back

Respectfully submitted:
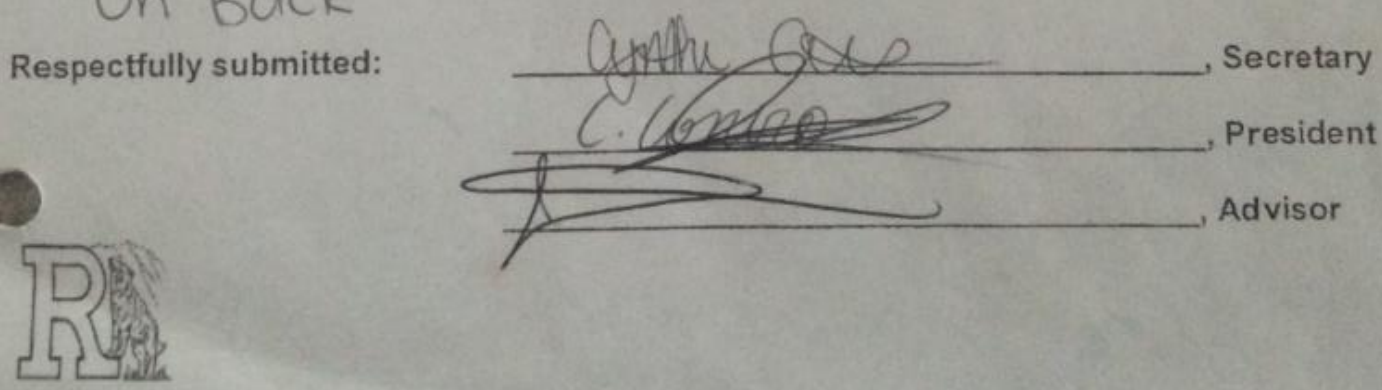


\section{Supporting Completion Materials BB: Wish List}

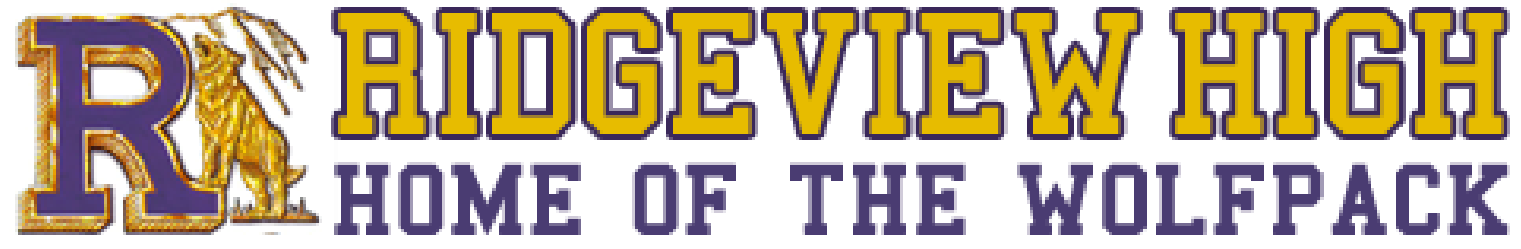




\section{BB. Wish List}

There are many items that would be included on my wish list for Ridgeview High School Agriculture Department. The list would include the following:

1. Adding a $2^{\text {nd }}$ teacher

2. A class set of Chromebooks

3. School Farm approval (I have a location, but have yet to get district approval)

4. Sheep surgical clipper blades

5. Tattoo pen for rabbits

6. New fair signs/decorations

7. A greenhouse

8. A more defined pathway (or 2) within the department

9. Beef clipper blades 
Supporting Completion

Materials CC: Agriculture

Department Operating

Budget \& Synopsis

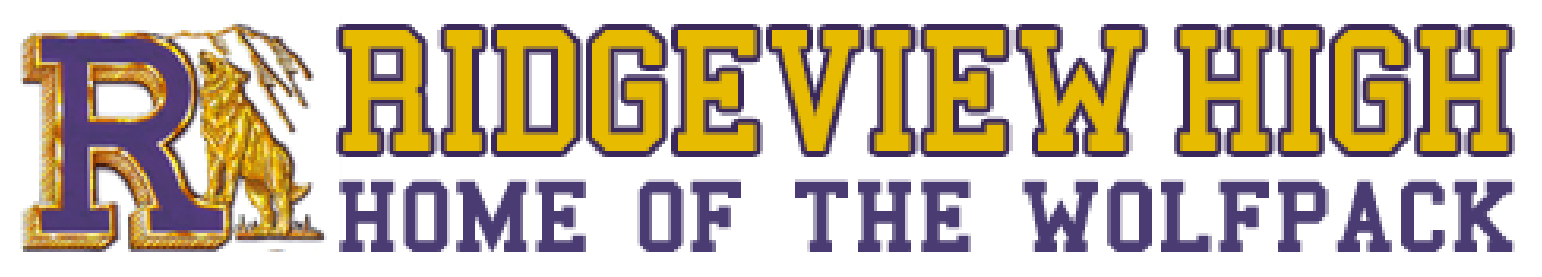




\section{Agriculture Department Operating Budget \& Synopsis}

Currently, I manage the AIG and ASB accounts. Attached you will find the budget used in my AIG application. For the ASB account, it is difficult to actually budget. In 2014 , the school changed accounting systems and are completely unable to tell club advisors the balance of their accounts, making it hard to track.'

Supporting Completion Material Attached 
C alifornia Department of E ducation

\section{AGRICULTURAL CAREER TE CHNICAL EDUCATION INCE NTIVE GRANT}

2015-16 APPLICATION FOR FUNDING

(Due Date: To be received in Regional Supervisor's O ffice by June 30, 2015)

DATE S OF PROJECT DURATION - JULY 1, 2015, TO JUNE 30, 2016

Ridgeview

Kern High School District

(School Site)

(D istrict)

Certification: I hereby certify that all applicable state and federal rules and regulations will be observed; that to the best of myknowledge, the in formation contained in th is application is corre $d$ and complete; and that the attached assurances are accepted as the basic conditions of the operations in th is project/program for local participation and assistance.

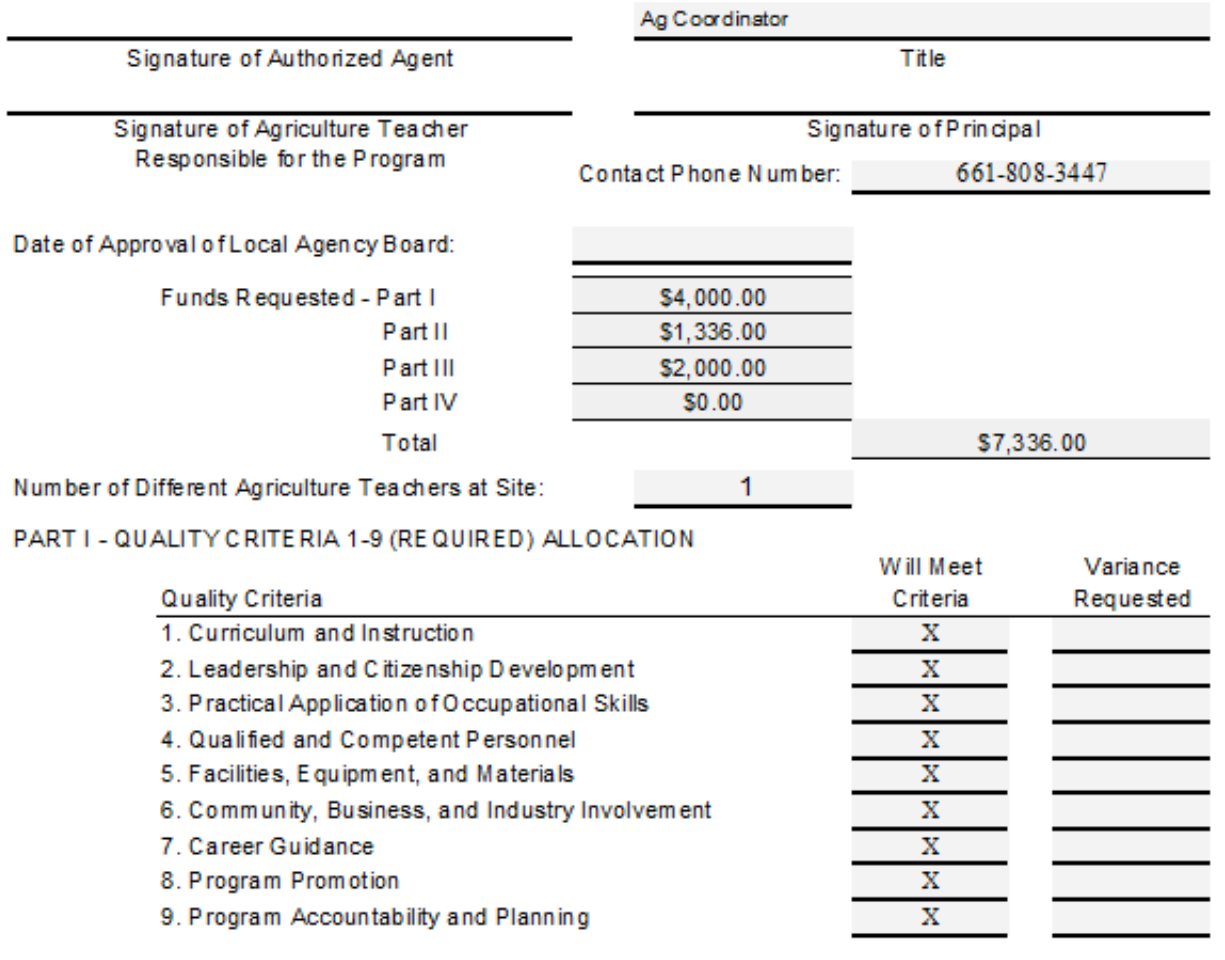

Formal Variance Request must be included if requesting a variance. A varian $\propto$ is a proposed plan for bringing the program into compliance with required quality criteria. Variances should result in compliance prior to the following year's application. All variances must be approved with the application. Non-complian $œ$ with the terms of the approved variance will result in a loss of funds. 
PART I - CONTINUED

Departmental Allocation: M eeting the criteria in PART I makes the program eligible for the following amounts based on the number of teachers in the program.

\begin{tabular}{lcc} 
Total Number of Teachers & Amount E ligible & Am ount R equested \\
\hline One Teacher or Less & $\$ 4,000$ & $\$ 4,000.00$ \\
Two Teachers & $\$ 4,500$ & \\
Three Teachers or M ore & $\$ 5,000$ &
\end{tabular}

PART II - PROGRAM ENROLLIMENT ALLOC ATION

Total Number of Students 2014-15 R2 Number Amount R equested

List $N$ umber from R2 Report (\$8/M ember) 167 $\$ 1,336.00$

PART III - QUALITY CRITERIA 10-11 (OPTIONAL) ALLOC ATION

Schools which qualify for a Departmental Allocation may apply for additional amounts for each specific Quality Criteria (10 and 11$)$ met.

* Amounts requested in Quality Criterion 10 will be the indicated amount for that criterion, multiplied by the full-time equivalent (FTE). To count a preparation period, the teacher must be teaching $\mathrm{Career}$ Technical E ducation courses in Agriculture for 50 percent or more of their teach ing periods.

* Amounts requested in Quality Criterion 11A will be the indicated amount for each teacher who was compensated a minimum of $\$ 2,000$ for year-round employment.

* Amounts requested in Quality Criterion $11 \mathrm{~B}$ will be the indicated amount for each teacher who is provided a project supervision period. Project periods will be counted if the teacher has a preparation period as part of the regular teaching day.

Number of FTE Agriculture Teachers at Site:

List the Names of the Agriculture Teachers:

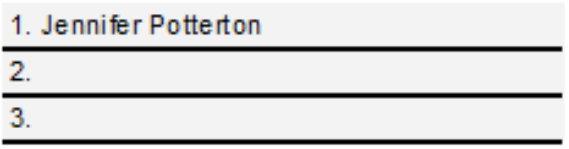

Criterion 10 - Student/Teacher Ratio

Criterion 11A - Year-Round E mployment

Criterion 11B - Project Supervision Period

TOTAL FUNDS REQUESTED PART IV
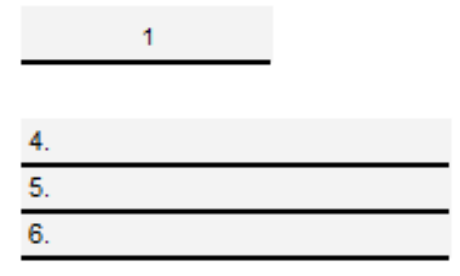

Number Meeting

C riteria

\begin{tabular}{c}
\hline 0 \\
\hline 1 \\
\hline 0
\end{tabular}

(1)

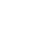

\section{PART IV - QUALITY CRITE RION 12 (OPTION AL) ALLOCATION}

Quality Criterion 12 Form is attached and all criteria has been met. If the answer is yes, list $\$ 7,500$ (funds requesting) in space to the right. 
PART V - FINANCIAL SCHEDU LE

Part A

\begin{tabular}{|c|c|c|c|c|c|}
\hline & & & A & B & C \\
\hline Line & $\begin{array}{l}\text { Acct. } \\
\text { No. }\end{array}$ & Classification & $\begin{array}{l}\text { Description of Item for } \\
\text { Which Funds Will be } \\
\text { Expended }\end{array}$ & \begin{tabular}{c|} 
Incentive \\
Grant Funds
\end{tabular} & $\begin{array}{l}\text { M atching } \\
\text { Funds }\end{array}$ \\
\hline 1 & 4000 & Books \& Supplies & & $6,336.00$ & $6,336.00$ \\
\hline 2 & & & Subtotal for 4000 & $\$ 6,336.00$ & $\$ 6,336.00$ \\
\hline 3 & \multirow[t]{6}{*}{5000} & \multirow{6}{*}{$\begin{array}{l}\text { Services and Other Operating } \\
\text { Expenses such as: Services of } \\
\text { Consultants, Sta ff Travel, and } \\
\text { Conferen ce; Rentals, Leases, and } \\
\text { Repairs; Bus Transportation }\end{array}$} & 1. Travel and Conf & $1,000.00$ & $1,000.00$ \\
\hline 4 & & & 2. & & \\
\hline 5 & & & 3. & & \\
\hline 6 & & & 4. & & \\
\hline & & & 5. & & \\
\hline 7 & & & 6. & & \\
\hline 8 & & & Subtotal for 5000 & $\$ 1,000.00$ & $\$ 1,000.00$ \\
\hline 9 & \multirow[t]{5}{*}{6000} & \multirow{5}{*}{$\begin{array}{l}\text { C apital Outlay. Includes Sites and } \\
\text { Improvements of Sites; Build ings and } \\
\text { Improvement of Build in gs; E quip ment }\end{array}$} & 1. & & \\
\hline 10 & & & 2. & & \\
\hline 11 & & & 3. & & \\
\hline & & & 4. & & \\
\hline 12 & & & 5. & & \\
\hline 13 & & & Subtotal for 6000 & $\$ 0.00$ & $\$ 0.00$ \\
\hline 14 & & & $\begin{array}{c}\text { Total for } 4000-6000 \\
\text { Lines } 2,8,13\end{array}$ & $\$ 7,336.00$ & $\$ 7,336.00$ \\
\hline
\end{tabular}

TOTAL 2015-16 In $œ$ ntive Grant Allocation:

Part B - Complete th is portion if a waiver of the matching requirem ent is requested:

\begin{tabular}{|r|r|l|l|l|l|l|}
\hline Line & $\begin{array}{c}\text { Act } \\
\text { No. }\end{array}$ & \multicolumn{1}{|c|}{ Classification } & $\begin{array}{l}\text { A } \\
\text { Description of Item for } \\
\text { Which Funds Were } \\
\text { Expended }\end{array}$ & $\begin{array}{c}\text { Incentive } \\
\text { Grant Funds }\end{array}$ & $\begin{array}{c}\text { Amount of } \\
\text { Salary and } \\
\text { Bene fits }\end{array}$ \\
\hline 15 & 1000 & Salaries & $\begin{array}{l}\text { Teachers' Summer } \\
\text { Service Salaries }\end{array}$ & & \\
\hline 16 & 1000 & Salaries & $\begin{array}{l}\text { Teachers' Salaries for } \\
\text { Project Supervision } \\
\text { Period }\end{array}$ & & \\
\hline 17 & 3000 & Benefits & $\begin{array}{l}\text { Benefits for the Above } \\
\text { Items (1000) }\end{array}$ & & & \\
\hline 18 & & TOTAL & & & $\mathbf{\$ 0 . 0 0}$ \\
\hline
\end{tabular}

TOTAL Amount of Waiver Requested: 


\section{Supporting Completion \\ Materials DD: Duties \& \\ Responsibilities Synopsis}

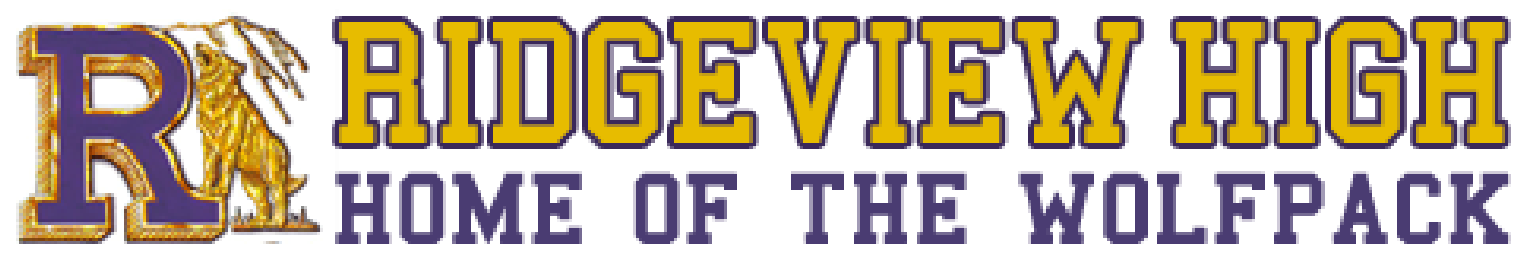




\section{DD. Duties \& Responsibilities Synopsis}

The Ridgeview High School Agriculture Department's only teacher, I am in charge of all the management of the department, including, but not limited to:

- Submitting R2 and related paperwork

- Managing AIG

- Correspond with Ag Advisory members

- Submit department PO and manage finances

- Submit field trip and absence request forms

- Fill out facility requests

- Register for all FFA events

- Book hotels for conferences

- Manage the department calendar

- Coach and advise student competitors and teams

- Management of school garden 
Supporting Completion

Materials EE: Chart of

Responsibilities

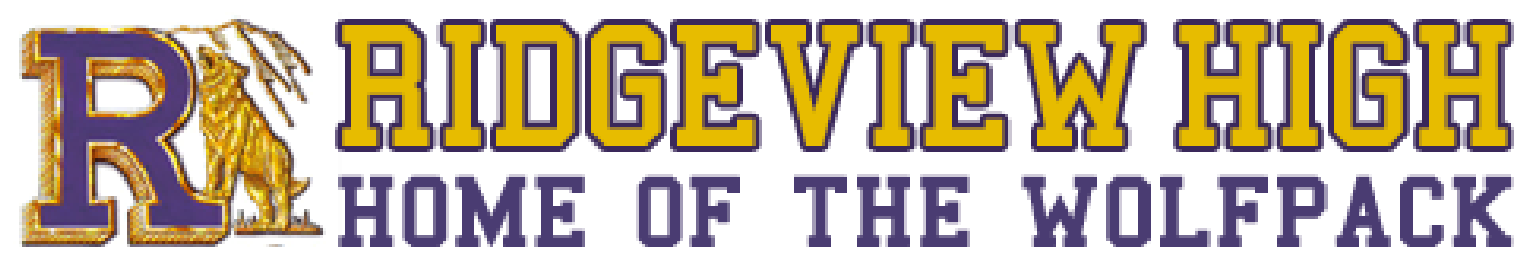




\section{EE. Chart of Responsibilities}

Supporting Completion Material Attached 


\section{RIDGEVIEW AGRICULTURE DEPARTMENT CHART OF RESPONSIBILITIES 2014-2015}

\begin{tabular}{|l|c|c|}
\hline \hline A. COURSE S & Potterton & \\
\hline 1. Agriculture Science & & \\
\hline a. Ag Earth Science & $X$ & \\
\hline b. AgBiology & $X$ & \\
\hline c. Vet Science & & \\
\hline d. Ag Sales & $X$ & \\
\hline
\end{tabular}

\begin{tabular}{|c|c|}
\hline B. DEPARTMENT MANAGEMENT & Potterton \\
\hline 1. Department Chairperson & $\mathrm{X}$ \\
\hline a. Agriculture Incentive Grant & $\mathrm{X}$ \\
\hline 1.) $\mathrm{Ag}$ Incentive Grant Application & $\mathrm{X}$ \\
\hline 2.) Ag Incentive Grant Expenditure Report & $\mathrm{X}$ \\
\hline 3.) Ag Incentive Grant Review Materials & $\mathrm{X}$ \\
\hline 4.) Ag Department Program Plan & $\mathrm{X}$ \\
\hline b. Ag Department Purchase Orders & $\mathrm{X}$ \\
\hline 1.) Ag Incentive Grant Account & $\mathrm{X}$ \\
\hline 2.) Perkins Grant Account & $\mathrm{X}$ \\
\hline 3.) FFA ASB account & $\mathrm{X}$ \\
\hline c. Agriculture Curriculum & $\mathrm{X}$ \\
\hline d. Agriculture Department Parents Club & $\mathrm{X}$ \\
\hline 1.) Parents Club Meetings & $\mathrm{X}$ \\
\hline a.) Ag Department Report & $\mathrm{X}$ \\
\hline b.) FFA Advisor Report & $\mathrm{X}$ \\
\hline & Potterton \\
\hline c.) FFA Member Participation & $\mathrm{X}$ \\
\hline e. Ag Department A dvisory Committee & $\mathrm{X}$ \\
\hline 1.) Advisory Committee Meetings & $\mathrm{X}$ \\
\hline 2.) Advisory Committee Meeting Announcement & $\mathrm{X}$ \\
\hline 3.) Advisory Committee Meeting Agenda & $\mathrm{X}$ \\
\hline 4.) Advisory Committee Meeting Minutes & $\mathrm{X}$ \\
\hline f. Agriculture Department M eetings & $\mathrm{X}$ \\
\hline 1.) Agriculture Department Meeting Agenda & $\mathrm{X}$ \\
\hline 2.) Agriculture Department Meeting Minutes & $\mathrm{X}$ \\
\hline 3.) Agriuclture Department Calendar & $\mathrm{X}$ \\
\hline
\end{tabular}




\begin{tabular}{|c|c|c|}
\hline 4.) Agriculture Department Records & $\mathrm{X}$ & \\
\hline 5.) Agriculture Department Supply Requisitions & $\mathrm{X}$ & \\
\hline \multicolumn{3}{|l|}{ g. Miscellaneous } \\
\hline 1.) Agriculture Teacher Inservice Record & $\mathrm{X}$ & \\
\hline 2.) Agriculture Teacher Summer Hours & $\mathrm{X}$ & \\
\hline 2. FFA Advisor & $\mathrm{X}$ & \\
\hline a. Student Data Sheets & $\mathrm{X}$ & \\
\hline 1.) Agriculture Department State R-2 Report & $\mathrm{X}$ & \\
\hline a.) Graduate Follow-Up Survey & $\mathrm{X}$ & \\
\hline 2.) Ag Department State FFA Roster Update & $\mathrm{X}$ & \\
\hline b. Activity Requests & $\mathrm{X}$ & \\
\hline 1.) Transportation Requests & $\mathrm{X}$ & \\
\hline 2.) Facility Requests & $\mathrm{X}$ & \\
\hline 3.) Master Calendar Requests & $\mathrm{X}$ & \\
\hline 4.) Fundraiser Requests & $\mathrm{X}$ & \\
\hline \multicolumn{3}{|l|}{ 3. Miscellaneous } \\
\hline a. Other Grant Applications & $\mathrm{X}$ & \\
\hline b. Other Scholarship Applications & $\mathrm{X}$ & \\
\hline \multicolumn{3}{|l|}{ 4. Equipment and F acilities } \\
\hline a. Garden & $\mathrm{X}$ & \\
\hline 1.) Maintenance and Repair of Facility & $\mathrm{X}$ & \\
\hline b. Agriculture Department Storage Facility $(\mathrm{H}-1)$ & $\mathrm{X}$ & \\
\hline 1.) Agriculture Department Vehicle & $\mathrm{X}$ & \\
\hline a.) Vehicle $M$ aintenance and Service & $\mathrm{X}$ & \\
\hline 2.) Agriculture Department Stock Trailer & $\mathrm{X}$ & \\
\hline 3.) Agriuclture Department Tractor & $\mathrm{X}$ & \\
\hline a.) Agriculture Department Equipment//mpliments & $\mathrm{X}$ & \\
\hline b. Degrees & $\mathrm{X}$ & \\
\hline 1.) Greenhand Degree Applications & $\mathrm{X}$ & \\
\hline 2.) Chapter Degree Applications & $\mathrm{X}$ & \\
\hline 3.) State Degreee Applications & $\mathrm{X}$ & \\
\hline 4.) American Degree Applications & $\mathrm{X}$ & \\
\hline \multicolumn{3}{|l|}{ c. Leadership Conferences } \\
\hline 1.) Chapter Officer Leadership Conference & $\mathrm{X}$ & \\
\hline 2.) Greenhand Degree Conference & $\mathrm{X}$ & \\
\hline 3.) Made For Excellence Conference & $\mathrm{X}$ & \\
\hline 4.) Advanced Leadership Conference & $\mathrm{X}$ & \\
\hline 5.) State FFA Leadership Conference & $\mathrm{X}$ & \\
\hline d. FFA Chapter Meetings & $X$ & \\
\hline
\end{tabular}




\begin{tabular}{|c|c|c|}
\hline 1.) FFA Chapter Officers & $X$ & \\
\hline a.) Chapter Officer M eetings & $X$ & \\
\hline 1.) Chapter Officer A pplications & $X$ & \\
\hline 2.) Chapter Officer Retreat & $X$ & \\
\hline b.) Chapter Program Of Work & $X$ & \\
\hline c.) Chapter Point Awards Program & $X$ & \\
\hline d.) Chapter Recruitment Program & $X$ & \\
\hline 2.) Chapter Banquets & $X$ & \\
\hline a.) Banquet Awards & $X$ & \\
\hline b.) Banquet Program & $X$ & \\
\hline c.) Banquet Slide Show & $X$ & \\
\hline 3.) Community Service & $X$ & \\
\hline 4.) Fundraisers & $X$ & \\
\hline 5.) Miscellaneous & & \\
\hline a.) FFA Welcome BBQ & $X$ & \\
\hline b.) FFA Week Activites & $X$ & \\
\hline c.) FFA Reward Trip (End of the Year) & $X$ & \\
\hline d.) Ag Expo (Tulare Farm Show) & $X$ & \\
\hline e.) FFA Jacket Orders & $X$ & \\
\hline f.) FFA State Finals Judging Contest Trip & & \\
\hline
\end{tabular}

\section{SUPERVISED AGRICULTURAL EXPERIENCE POTTERTON}

\begin{tabular}{|l|c|l|}
\hline 1. SAE Project Supervision & $\mathrm{X}$ & \\
\hline a. Livestock & $\mathrm{X}$ & \\
\hline 1.) Beef Cattle & $\mathrm{X}$ & \\
\hline 2.) Dairy Cattle & $\mathrm{X}$ & \\
\hline 3.) Goats & $\mathrm{X}$ & \\
\hline 4.) Sheep & $\mathrm{X}$ & \\
\hline 5.) Swine & $\mathrm{X}$ & \\
\hline b. Small Animals & $\mathrm{X}$ & \\
\hline 1.) Poultry & $\mathrm{X}$ & \\
\hline 2.) Rabbits & $\mathrm{X}$ & \\
\hline c. Agriculture Mechanics & $\mathrm{X}$ & \\
\hline d. Ornamental Horticulture & $\mathrm{X}$ & \\
\hline e. Agricultural Work Experience & $\mathrm{X}$ & \\
\hline 2. Fairs and Shows & & \\
\hline a. SAE Project Supervision and Transportation & $\mathrm{X}$ & \\
\hline b. Student Supervission and Transportation & $\mathrm{X}$ & \\
\hline c. Chapter Supply and Equipment Transportation & $\mathrm{X}$ & \\
\hline
\end{tabular}




\begin{tabular}{|l|l|l|}
\hline d. Fair and Show Entries and Fees & $\mathrm{X}$ & \\
\hline e. Fair and Show Entry Tagging & $\mathrm{X}$ & \\
\hline 1.) Kern County Fair & $\mathrm{X}$ & \\
\hline 2.) Desert Empire Fair & $\mathrm{X}$ & \\
\hline 7.) M iscelaneous Other Exhibits & $\mathrm{X}$ & \\
\hline 3. SAE Project Proficiencies & & \\
\hline a. Chapter Proficiencies & $\mathrm{X}$ & \\
\hline 1.) Chapter Proficiency Scoring & $\mathrm{X}$ & \\
\hline b. Sectional Proficiencies & $\mathrm{X}$ & \\
\hline 1.) Sectional Proficiency Scoring & $\mathrm{X}$ & \\
\hline c. Regional Proficiencies & $\mathrm{X}$ & \\
\hline 1.) Regional Proficiency Scoring & $\mathrm{X}$ & \\
\hline d. State Proficiencies & $\mathrm{X}$ & \\
\hline 1.) State Proficiency Scoring & $\mathrm{X}$ & \\
\hline e. National Proficiencies & $\mathrm{X}$ & \\
\hline 1.) National Proficiency Scoring & $\mathrm{X}$ & \\
\hline
\end{tabular}

\begin{tabular}{|l|c|c|}
\hline E. JUDG ING TEAMS & Potterton & \\
\hline 1. Leadership Teams & & \\
\hline a. FFA Opening and Closing Ceremonies & & \\
\hline 1.) Officer Opening and Closing Ceremonies & $\mathrm{X}$ & \\
\hline 2.) Open Opening and Closing Ceremonies & $\mathrm{X}$ & \\
\hline 3.) Freshmen Opening and Closing Ceremonies & $\mathrm{X}$ & \\
\hline b. FFA Public Speaking & & \\
\hline 1.) FFA Creed Speakers & $\mathrm{X}$ & \\
\hline 2.) Prepared Public Speaking & $\mathrm{X}$ & \\
\hline 3.) Job Interview & $\mathrm{X}$ & \\
\hline \multicolumn{2}{|c|}{ POTTERTON } \\
\hline Miscellaneous & & \\
\hline a. Banking & $\mathrm{X}$ & \\
\hline b. Coops & $\mathrm{X}$ & \\
\hline c. Novice Records & $\mathrm{X}$ & \\
\hline e. Best Informed Greenhand & $\mathrm{X}$ & \\
\hline
\end{tabular}


Supporting Completion Materials FF: Substitute Teacher Procedures and Plans

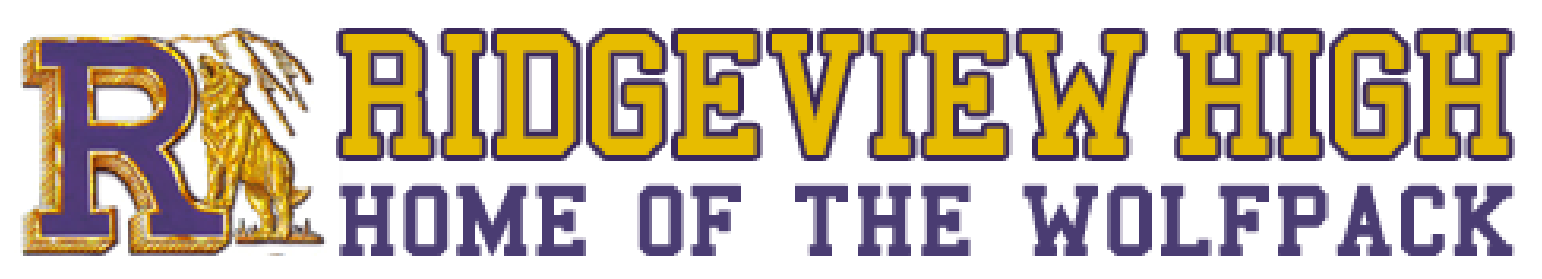




\section{FF. Substitute Teacher Procedures and Plans}

For my substitute plans, I have a binder. The binder starts with my general sub plans, which are attached. Then it has tabs for each class with their assignment for today. I also try to make the handouts for the students self-explanatory, so the students can do the work with little instruction.

Supporting Completion Material Attached 
Thank you for taking my classes! If you have any questions or need information, please contact me via call or text message at the cell phone number listed above or email me, which may not have as quick of a response.

I know this is long, but please take a moment to read through this so that you are acquainted with my clasgroom

\section{*Class Schedule:}

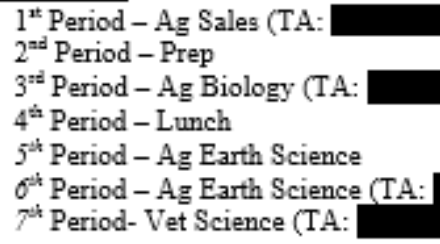

*Bell schedule is posted on the bookcase by my desk.

*Take roll at the beginning of class, please count tardy students (if they aren't in their assigned seats please feel free to mark them tardy).

Gecko: Students are not to handle the gecko (you probably will hardly see it) and I will take care of feeding it

Projector \& videos: You should not need to use the projector at all during the time I am gone, no movies please.

Teacher's desk: No students should sit at my desk at any time.

TA's desk: The desk on the side of the room is for the TAs.

Students tend to spend breaks and store items in my classroom; it is completely up to you if you feel comfortable with this. (show this to a kid if they argue with you about it)

- All students are assigned seats - seating charts are on the clipboard, they must sit in their assigned seats, if they refuse to, I consider this a behavior issue.

- No students are allowed on any of the computers in the room other than TAs (no student should sit in the desk chairs either)

- Students may not enter the back work room and kitchen areas at any time (work rooms should be locked).

- Announcements are read at the beginning of $2^{\text {ed }}$ period over the loud speaker.

- NO: CD players, iPods or cell phones (without your permission) - if you see any of these items, confiscate them immediately. Use the "item confiscation" slips in the top drawer of my desk to write up the 
Email: Jennifer_potterton@kernhigh.org item, and then take the paper with the item to the dean's office as soon as possible or call them to come pick it up for me (x 62040).

- If students need to use the restroom or get something they forgot from their locker, I usually let them go. The pass is by the door. Do not let more than one student out at a time. If students need to go out too often, say no. No students can go out during the first and last 15 minutes of class.

- I have a list of school phone numbers posted above the phone.

- If you need help from another teacher, Mike Braiser (room 1106) is really helpful

- If students are behavior problems, hand them a clipboard from the table by my desk and instruct them to go to any of the following science teachers rooms (I usually use Mike or Jenny), they will retum with the form filled out and hopefully the behavior curbed. Please keep the form for me to file.

- Mike Braiser, 1106

- Jenny Berry, 1105

- Sam Yoon, 1115

- Stephan Kiouses, 1111

- Students may use my notebooks by the TA desk to check their notebook.

- They may NOT use the radio

\section{Thank you for spending time in my classroom!!!!!!!}


Supporting Completion Materials GG: Program Completers

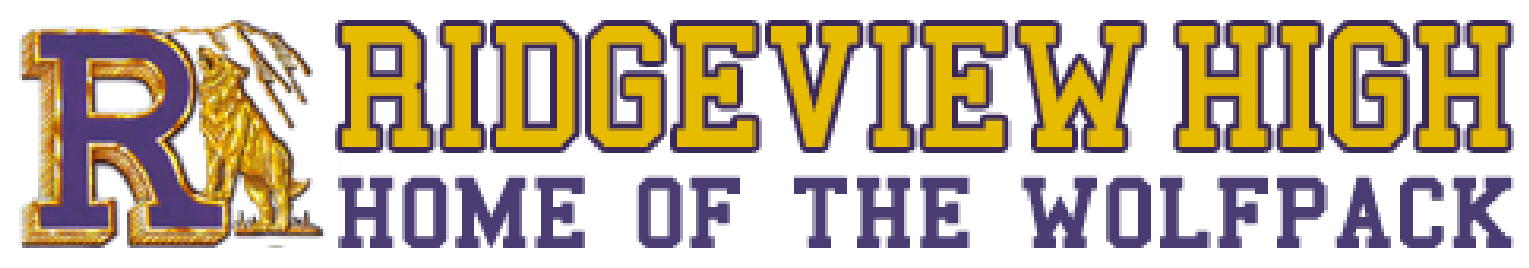




\section{GG. Program Completers}

Supporting Completion Material Attached 


\section{Program Completer Standards}

To insure the greatest possible standards of excellence in the Ridgeview Agriculture Program, the following areas must be completed in order for a student to be considered as a "Program Completer":

1.) A student must maintain a " Average in all Agriculture course work.

2.) A student must be involved for four (4) years in the FFA Program.*

- The student must be proficient in Parliamentary Procedure.

- The student must have given a six minute speech or led a group discussion for 40 minutes on a topic relating to the FFA.

- They must have taken part in 16 FFA activities. $\sqrt{ } 5$ of these activities must be above the chapter level.

3.) A student must be involved in an expanding SOE Project for three years, *

- The student must have earned or productively invested at least $\$ 1000.00$, or worked at least $\$ 00$ hours in excess of scheduled class time.

4.) Maintain an Agriculture Portfolio containing:

- Letter of Introduction

- Career Development Package.

$\sqrt{ }$ Application for employment

$\sqrt{ }$ Letter of recommendation

$\sqrt{ } \quad$ Resume

- Four work samples with summaries.

- Writing Sample.

- Supervised Occupational Experience Evaluation.

*This information must be verified in the students FFA Recordbook. 


\section{Supporting Completion \\ Materials HH: Dual Enrollment}

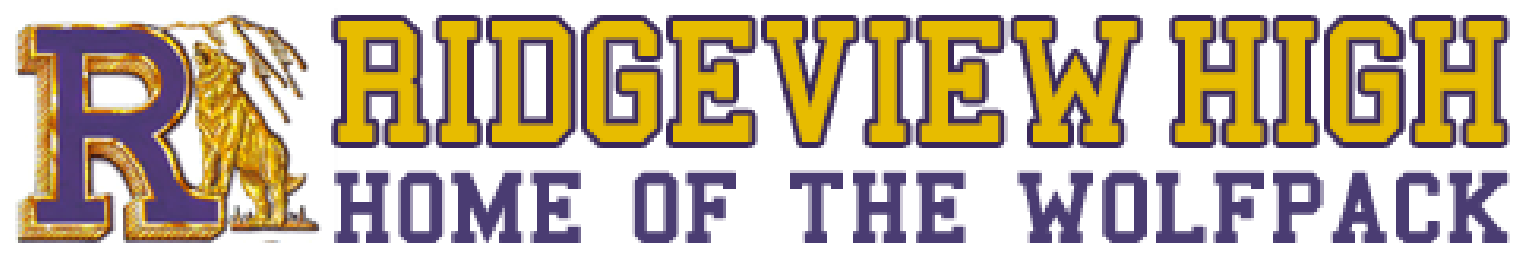




\section{HH. Dual Enrollment}

Supporting Completion Material Attached 


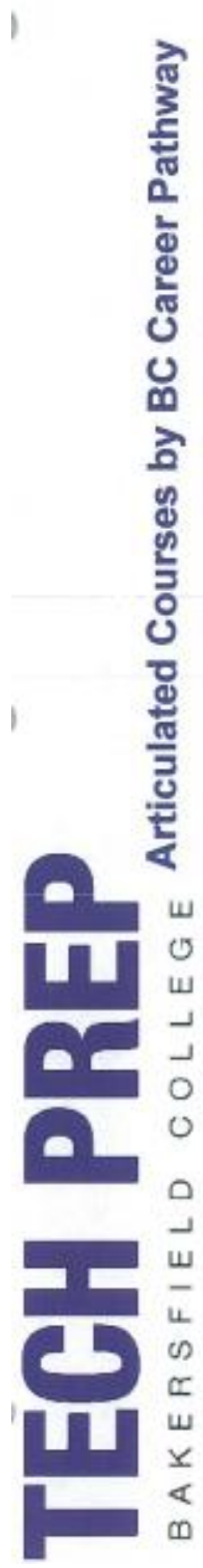

\begin{tabular}{|c|c|c|c|c|c|c|c|c|}
\hline CosøM & $\bullet$ & $\bullet$ & $\bullet$ & & & $\bullet$ & $\bullet$ & $\bullet$ \\
\hline УaItนHS & - & & & $\bullet$ & & & $\bullet$ & \\
\hline 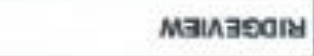 & $\bullet$ & & - & & & & $\bullet$ & \\
\hline गCO ASHY & & $\bullet$ & & & & & $\bullet$ & \\
\hline HLYON & - & $\bullet$ & & $\bullet$ & & & & \\
\hline GINOW BaIW & - & $\bullet$ & & & & $\bullet$ & & \\
\hline aNVาบชมอพ & - & & $\bullet$ & $\bullet$ & & - & $\bullet$ & \\
\hline Aมะ3ar7 & & $\bullet$ & & & & & & \\
\hline 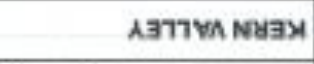 & - & $\bullet$ & $\bullet$ & $\bullet$ & & $\bullet$ & $\bullet$ & $\bullet$ \\
\hline \multicolumn{9}{|l|}{ (OSHO) BONבONבdBONI } \\
\hline ลNYานภH & $\bullet$ & $\bullet$ & $\bullet$ & $\bullet$ & & - & $\bullet$ & \\
\hline 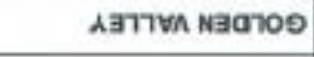 & $\bullet$ & & - & & & - & & \\
\hline УaUNOAd & $\bullet$ & $\bullet$ & $\bullet$ & & & & & \\
\hline NLW y & & & $\bullet$ & $\bullet$ & & & $\bullet$ & \\
\hline าาเหนอำ & $\bullet$ & - & - & $\bullet$ & & - & & \\
\hline ONGTad & $\bullet$ & & $\bullet$ & $\bullet$ & & & - & \\
\hline SHE & $\bullet$ & $\bullet$ & & & & $\bullet$ & - & $\bullet$ \\
\hline NANYG & $\bullet$ & & $\bullet$ & & & $\bullet$ & $\bullet$ & $\bullet$ \\
\hline 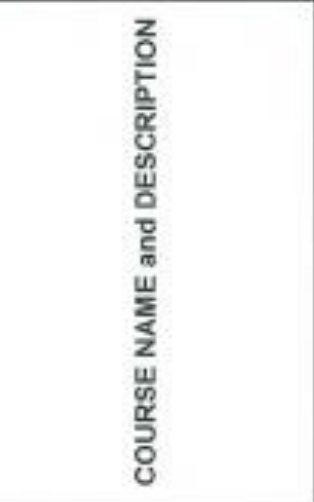 & 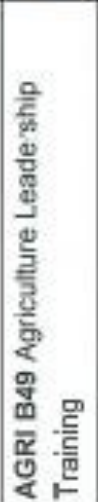 & 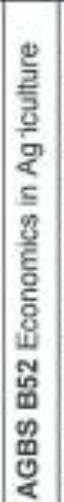 & 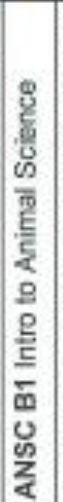 & 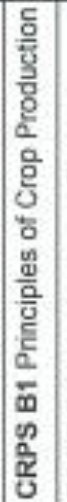 & 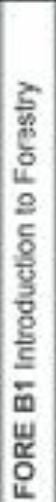 & 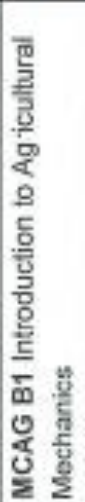 & 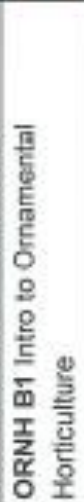 & 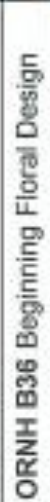 \\
\hline
\end{tabular}


Supporting Completion Materials II: Department Reimbursements

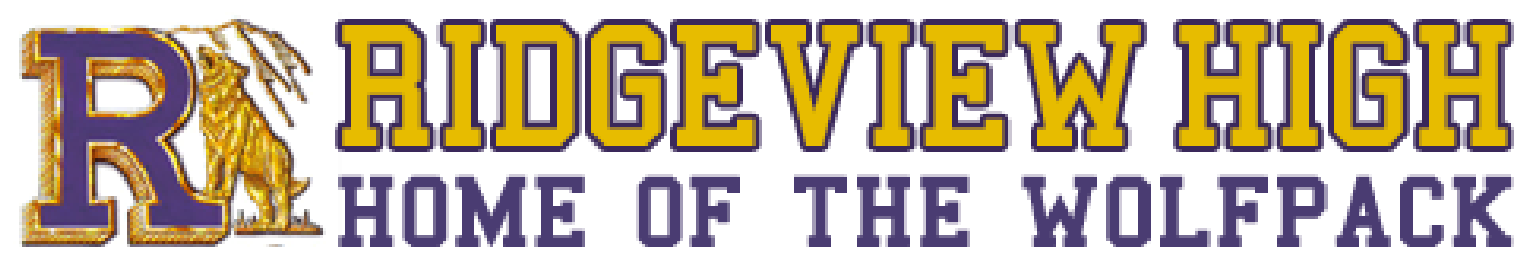




\section{Department Reimbursements}

Supporting Completion Material Attached 
Request for Payment and Disbursement Authoriration (Check Roquest) 10.2113 Date approved by Cho or Organiman $10 / 2,13$ Anount 19.95 Mal Aocosunt Number Account Name $F F A$ Vendor Nic Purchase Onder:

Payeo Jering Potheritor

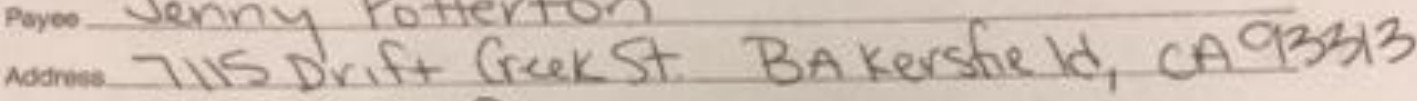
mone Na. $8755-245-15100$ Fax No Federa 10 o (Social security $\quad 1213$ Pupose MPRtanoy smoKS

Student C. Congthered

Date:

3thiebc/Activies Director,

Advisor

$10 / 2 / 13$

Principal

Representative

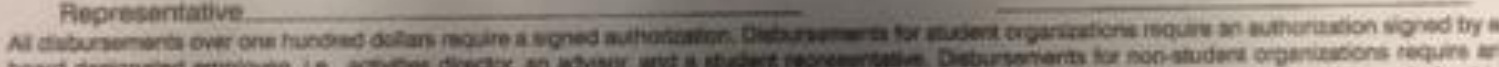

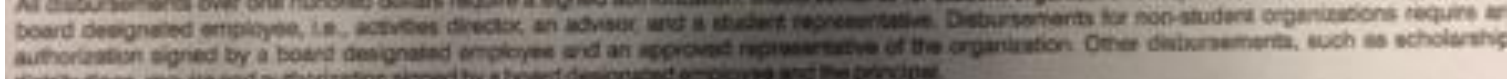
Checks will not be processed without authortaed algnaturea. 10hoboro

Disthis copr

293 


\title{
Smart\&.Final.
}

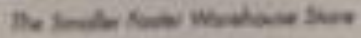

* wicone is ar basentiold Stirs ". itire,$j 44$

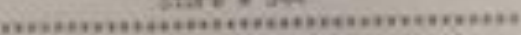

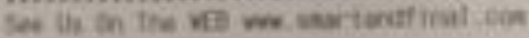

Camileri Nertal.

pati gait/2

It: $35-4: 44$

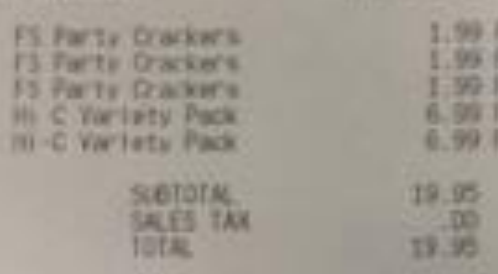

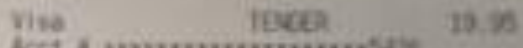

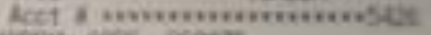

Heth coce obar?

cos Pate 91

cor

OMer $\quad \infty$

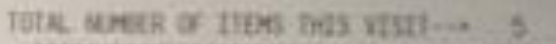

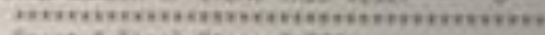
Sart I Iinal stare 4 J4

3200 oite Lre

Raverstuld of sasos

gatr ounvas.

$\operatorname{Tt} 5.15-272=$

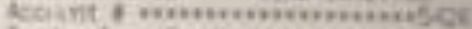

fercer trot cresit

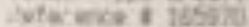

spona cose oseste

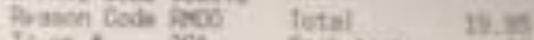

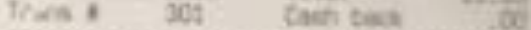

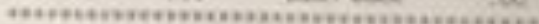

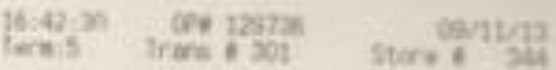

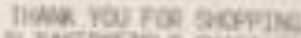

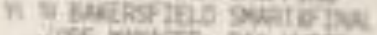

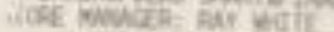

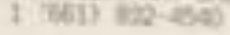

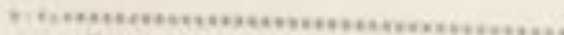

w want to knoe wour theint

so con herve vou butfer

casciele our antaner surn

we be wot red ta wing

4:; ? SmartCash Card

Plu ne visit

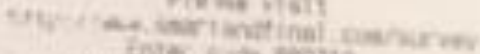

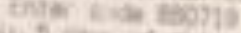

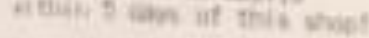

